\title{
Ray tracing in a turbulent, shallow-water channel
}

\author{
Bjerrum-Niese, Christian; Lützen, René; Jensen, Leif Bjørnø
}

Published in:

Acoustical Society of America. Journal

Link to article, DOI:

$10.1121 / 1.422455$

Publication date:

1998

Document Version

Publisher's PDF, also known as Version of record

Link back to DTU Orbit

Citation (APA):

Bjerrum-Niese, C., Lützen, R., \& Jensen, L. B. (1998). Ray tracing in a turbulent, shallow-water channel. Acoustical Society of America. Journal, 103(5), 2751-2751. https://doi.org/10.1121/1.422455

\section{General rights}

Copyright and moral rights for the publications made accessible in the public portal are retained by the authors and/or other copyright owners and it is a condition of accessing publications that users recognise and abide by the legal requirements associated with these rights.

- Users may download and print one copy of any publication from the public portal for the purpose of private study or research.

- You may not further distribute the material or use it for any profit-making activity or commercial gain

- You may freely distribute the URL identifying the publication in the public portal

If you believe that this document breaches copyright please contact us providing details, and we will remove access to the work immediately and investigate your claim. 


\title{
Session 2aPLa
}

\section{Plenary Lecture}

Lawrence A. Crum, Chair

Applied Physics Laboratory, University of Washington, 1013 NE 40th Street, Seattle, Washington 98105-6698

Chair's Introduction-8:00

\section{Invited Paper}

\section{8:05}

2aPLa1. Noninvasive ultrasonic surgery. Gail R. ter Haar (Phys. Dept., Royal Marsden Hospital, Sutton, Surrey SM2 5PT, UK, gail@icr.ac.uk)

The millimeter and submillimeter wavelengths of megahertz ultrasound in tissue mean that this form of energy can be brought to a tight focus in the body at a distance from the acoustic source. If the energy carried by the ultrasonic beam is sufficiently high, then it is possible to destroy tissue lying within the focal region, while sparing adjacent and overlying tissues. Typical peak intensities at the focus for ablation of tissues using 1-2 s exposure times are $1 \mathrm{~kW} / \mathrm{cm}^{2}$. For a focused bowl transducer with focal length $15 \mathrm{~cm}$, $8 \mathrm{~cm}$ in diameter, operating at $1.7 \mathrm{MHz}$, the focal region is ellipsoidal in form, with axial length $15 \mathrm{~cm}$ and diameter $1.5 \mathrm{~mm}$. A number of different exposure methods may be used to ablate clinically useful tissue volumes. Focused ultrasound is capable of selectively destroying tissue at depth without the need to expose the site of interest surgically. The mode of tissue destruction is primarily thermal in origin, temperatures in excess of $60^{\circ} \mathrm{C}$ being induced very rapidly. This type of cellular damage is capable of destroying both normal and malignant cells, and of occluding selected blood vessels. Potential applications for this technique lie, among others, in urology, oncology, and fetal medicine.

\section{Session 2aAAa}

\section{Opera House Acoustics Workshop-CIARM, MCHA, Architectural Acoustics and Musical Acoustics: Sound Field for Singers and Orchestra, Part 2}

\author{
Christopher N. Blair, Cochair \\ Artec Consultants, Inc., 114 West 26th Street, New York, New York 10001 \\ Anders C. S. Gade, Cochair \\ Department of Acoustic Technology, Technical University of Denmark, Building 352, DK-2800 Lyngby, Denmark \\ Chair's Introduction-7:45

\section{Contributed Papers}

\section{$7: 50$}

2aAAa1. On the preferred delay time of a single reflection for cellists. Shin-ichi Sato, Yoichi Ando (Grad. School of Sci. and Technol., Kobe Univ., Rokkodai, Nada, Kobe, 657 Japan), and Saho Ota (Kobe Univ., Kobe, 657 Japan)

In order to attain a knowledge of designing the stage enclosure in a hall, the subjective preference of sound fields with a single reflection for alto-recorder players has been reported [I. Nakayama, Acustica 54, $217-$ 221 (1984)]. In this study, subjective preference judgments of sound fields with a single reflection were performed by cello players. It is examined whether or not preferred delay time of reflection is dependent on the effective duration $\left(\tau_{e}\right)$ of the autocorrelation function (ACF) of the music signals performed by each player and the amplitude of the reflection. [Work was supported by the Grant-in-Aid for Scientific Research JSPS.]

8:05

2aAAa2. A study of improvements to acoustical conditions for singers. Dennis Noson (107 North 77th St., Seattle, WA 98103, noson@worldnet.att.net)

While numerous recent studies have been reported on improving stage acoustics for orchestral performers, the preferred acoustical conditions on performing stages for singers has received limited attention in the past 30 
years, with pioneering studies by Nakamura and Marshall. A series of acoustical modifications has been proposed for a Seattle church to improve the acoustics for both the listeners and the performing choir. An investigation was made, within the constraints of the existing acoustical environment of the church, to determine what direction the acoustical modifications should take to improve conditions of ensemble and balance among the singers while simultaneously improving the sound field for the audience. Acoustical conditions were modified both by adding temporary reflecting panels and introducing simulated reflections with strategically located loudspeakers. The results indicate a potential for noticeable improvement in the existing chancel acoustics, with most of the benefit applying to the singers rather than the listeners. Comparison will be made between the measured changes to the sound field and the predicted response of the singers using Ando's theory of subjective preference.

\section{8:20}

2aAAa3. Some recent experiences with the acoustical design of orchestra pits. Dennis Paoletti, Kurt Graffy, and Larry Tedford (Paoletti Assoc., Inc., 40 Gold St., San Francisco, CA 94133)

A number of recent projects including work for the San Francisco and Houston Operas have included acoustical designs for new and/or renovated orchestra pits. This paper will review some of those projects, their criteria, design, measurements, and evaluations.

\section{8:35}

2aAAa4. The virtual orchestra; pedagogical and creative issues. Frederick W. Bianchi (Humanities Dept., Music Div., Worcester Polytechnic Inst., 100 Institute Rd., Worcester, MA 01609) and Richard H. Campbell (Worcester Polytechnic Inst., Worcester, MA 01609)

The interactive multichannel computer music system known as the "Virtual Orchestra" has been used several times in professional opera and theater as an alternative to a live pit orchestra. The technical issues associated with this emerging technology, and the logistical problems of implementing it are presented in a companion paper. This paper describes the equally important issues regarding the creative and pedagogical impact this technology will have on the industry. In particular, the paper suggests that many of the fundamental premises of opera production will change as the industry begins to retool. This would include the way opera is rehearsed and performed, how it is created and disseminated, and how it will adjust to the changing demographics of the country. In addition, the paper addresses the notion of the technologist/musician and examines the role of education and professional training in computer music and musical acoustics.
8:50

2aAAa5. The virtual orchestra; acoustical and audio engineering issues. Richard H. Campbell (Elec. and Comput. Eng. Dept., Worcester Polytechnic Inst., 100 Institute Rd., Worcester, MA 01609) and Frederick W. Bianchi (Worcester Polytechnic Inst., Worcester, MA 01609)

The multichannel MIDI system known as the "Virtual Orchestra" has been used several times in the pit for the performance of opera, and recently demonstrated at an ASA regional chapter meeting in Boston. This paper discusses technical issues such as the selection of the types of loudspeakers and their placement in the pit, the amplifier power requirements, and the sound level calibration for each instrumental section based on a mezzo-forte norm. "Wolf' tones caused by understage extension cavities and other pit deficiencies can be easily modified within the MIDI computer program or with external equalization. Ideas are presented for the use of this new tool with respect to an acoustic freedom heretofore unavailable to orchestrators of musical theater works. The computer operator's ability to follow in real time the conductor's direction in nuance and tempo guarantees a cohesive performance synchronized with the singers and the dramatic action.

\section{9:05}

2aAAa6. The effect of early reflection on players in a concert hall. Kanako Ueno (Dept. of Architecture, Tokyo Univ., 7-3-1 Hongou, Bunkyo-ku, Tokyo, Japan, ueno@iis.u-tokyo.ac.jp), Fumiaki Satoh (Chiba Inst. of Technol., Narashino-shi, Chiba, Japan), Hideki Tachibana (I.I.S. of Tokyo Univ., Minato-ku, Tokyo, Japan), Takahiko Ono (Ono-Sokki Co., Midori-ku, Yokohama-shi, Kanagawa, Japan), and Mariko Senju

In order to investigate the effect of hall response on players, field measurements on the stage of a concert hall and laboratory experiment using digital simulation technique were performed. In the field experiment, the subject, a professional violinist, was asked to play and to make comments on her acoustical impression of five points on the stage. As a physical measurement, impulse responses were obtained at the same points by using omni-directional loudspeakers as a sound source and an omni-directional microphone and directional microphones as receivers. As a result, it has been found that not only the strength of the early reflections but also their direction influences the subject's impression. In the laboratory experiment (anechoic chamber), the sound field was modeled and synthesized by using a 13 channel reproduction system; ambient reverberation judged as being natural was provided by simple digital reverberators and different strength and direction of early reflections were obtained by real-time convolvers. For a constant value of reverberation, several conditions with a different level and direction of the early reflections were created. For each condition, the violin player was asked to make similar judgments as in the field experiment. The results of two experiments were examined. 


\title{
Session 2aAAb
}

\section{Opera House Acoustics Workshop-CIARM, MCHA, and Architectural Acoustics: Sound Field for the Audience, Part 2}

\author{
Yoichi Ando, Cochair \\ Graduate School of Science and Technology, Kobe University, Rokkodai, Nada, Kobe, 657 Japan \\ Michael Barron, Cochair \\ Department of Architecture and Civil Engineering, University of Bath, Bath BA2 7AY, England
}

Chair's Introduction-9:20

Invited Papers

2aAAb1. Boxes and sound quality in an Italian opera house. Alessandro Cocchi, Massimo Garai, and Carla Tavernelli (DIENCA, viale Risorgimento 2, 40136 Bologna, Italy, massimo.garai@mail.ing.unibo.it)

The sound field in the boxes of an 18th century Italian opera house was studied by means of binaural measurements and computer simulations. The differences in listening between the boxes and the stall is best described by the values of ITDG, clarity, and spatial criteria resulting from the measurements. The periodic variation of the ITDG along the boxes depends also on the reflections coming from the ceiling. The best boxes for clarity and spatial impression are found in very different positions. Computer simulation supports these conclusions and helps in understanding the two different mechanisms by which the boxes influence the sound field in the hall: the equivalent sound absorption of the boxes and the periodic structure of the box-covered walls. A validated computer model allows one to forecast how the sound field in the historical theater could be if some interior lining were added to the boxes, trying to restore the velvet lining which was supposed to exist in ancient times.

2aAAb2. Measurements of the polarization of sound energy at the mouth of boxes of an Italian opera house. Domenico Stanzial, Davide Bonsi, and Nicola Prodi (CIARM c/o CNR-Cemoter Acoust. Dept., via Canal Bianco 28, I-44044 Ferrara, Italy)

The measurement of a newly discovered property of sound energy, the polarization [Stanzial et al., J. Acoust. Soc. Am. 99, $1868-1876$ (1996)], has been carried out for the first time inside an opera house. Since energy polarization is due to the distributions of wavefronts passing through the measurement point, the analysis of this quantity allows one to obtain a precise picture of the effect of sound reflections at the receiving location. The highly symmetric architectural structure of an Italian opera house has been thus investigated at the opening of some boxes and the energy polarization has been compared with the sightline from each box to the stage. Furthermore, a third octave band frequency analysis in the range $100-5000 \mathrm{~Hz}$ has been accomplished that allows the visualization of the frequency-dependent behavior of the reflections' distribution.

\section{0:05}

2aAAb3. Measurements in an opera house: Comparison between techniques and instrumentation. Patrizio Fausti, Roberto Pompoli (Dipartimento di Ingegneria, Univ. of Ferrara, Via Saragat 1, I-44100 Ferrara, Italy), and Angelo Farina (Univ. of Parma, Parma, Italy)

In room acoustics, many objective parameters to quantify subjective impression have been introduced. These quantities can be measured using a wide variety of powerful tools and instrumentation. The results can be influenced by the measurement techniques and instrumentation used. Furthermore, the results also depend on the position and condition of the hall in which the measurements are made. With the aim to obtain a procedure to qualify a room acoustically, and in particular an opera house, which gives comparable and reproducible results all over the world, an extensive measurement campaign was made. The procedure takes into account the measurement techniques, the instrumentation, the measurement positions, and the condition of the audience and the stage. Comparisons were made both between different tools (real-time analyzer, DAT, PC-board) and different kinds of acoustic excitation (stationary, impulsive, pseudorandom). Furthermore, comparisons were made in different conditions of the stage and pit (e.g., empty, with the presence of orchestra tools and/or musicians). In this paper the results of the measurement campaign are presented and a proposal is submitted for the discussion. 


\title{
Contributed Paper
}

10:25

2aAAb4. Individual subjective preference of listeners for vocal music sources in relation to the subsequent reverberation time of sound fields. Hiroyuki Sakai (Grad. School of Sci. and Technol., Kobe Univ., Rokkodai, Nada, Kobe, 657 Japan), Hiroshi Setoguchi (Miyama Conceru, Kagoshima, 899-66 Japan), and Yoichi Ando (Kobe Univ., Kobe, 657 Japan)

The purpose of this study is to evaluate subjective preference of a simulated sound field by listeners in changing subsequent reverberation time $T_{\text {sub }}$ using vocal music sources. Previously, a great deal of effort has been made on the study using music or speech [for example, Ando et al.,
Acustica 50, 134-141 (1982); J. Acoust. Soc. Jpn. 39, 89-95 (1983)]. In addition to subjective preference judgments, the $\alpha$-waves' range of the continuous brain waves (CBW) in relation to the specialization of human cerebral hemispheres is also evaluated [C. Chen and Y. Ando, J. Archit. Plann. Environ. Eng., AIJ 489, 73-80 (1996)]. In this paper, subjective evaluation using vocal music sources, which is often played in concert halls and opera houses, has been examined. Subjective preference tests were conducted changing $T_{\text {sub }}$, which is one of the four objective parameters in relation to subjective preference of sound fields. Individual differences of subjective preference as well as global preference are discussed. [Work was partially supported by the Ministry of Education, Grant-in-Aid for Scientific Research (C), 9838022, 1997.]

TUESDAY MORNING, 23 JUNE 1998

VASHON ROOM (W), 8:00 TO 10:45 A.M.

\section{Session $2 \mathrm{aAB}$}

\section{Animal Bioacoustics: Insect, Insectivore and Avian Acoustics}

\author{
Timothy G. Forrest, Chair \\ Department of Biology, University of North Carolina, One University Heights, Asheville, North Carolina 28804
}

\section{Invited Papers}

\begin{abstract}
8:00
2aAB1. Scale effects as constraints in insect sound communication. Henry Bennet-Clark (Dept. of Zoology, Oxford Univ., S. Parks Rd., Oxford OX1 3PS, UK)

Calling insects have problems of optimizing range and maintaining the specificity of the call. Higher frequencies are more easily refracted and reflected by objects in the environment. Smaller insects have less muscle power and also, because of the small sound source size, higher frequencies are radiated more efficiently than lower frequencies. In open air or water, the sound spreads spherically and decays by the inverse square law. If the sound can be confined to a sheet it decays as the inverse of range while within a rod it decays due to viscous losses; such calls are usually rather simple pulses and rely on initial time of arrival because of multiple pathlengths in the environment. With airborne sounds, those in the range from 1 to $10 \mathrm{kHz}$ tend to have sustained pure-tone components and a specific pattern of pulses which propagates well; but with higher frequencies pulses tend to become briefer and, once again, to rely on time of arrival of the onset.
\end{abstract}

\section{$8: 25$}

2aAB2. Acoustic detection and identification of insects in soil. Richard Mankin (USDA-Agric. Res. Service, Ctr. for Medical, Agricultural, and Veterinary Entomology, P.O. Box 14565, Gainesville, FL 32604)

There is considerable practical need for user-friendly, inexpensive devices that detect and quantify insect populations in environments hidden from visual observation. One approach that has been used with varying success has been to detect the insects through the sounds or vibrations they generate for communication or through noises that are produced incidentally during feeding and general movement. The ability to precisely monitor soil insect populations is limited by interference from background noise and the high rate of attenuation of sound in soil. This paper describes experiments using different sensors and analysis techniques for detection of insects in soil in an agricultural environment. Results from different sensors are compared, and the spectral and temporal patterns that can be used to distinguish the target insects from noise and nontarget soil organisms are discussed. The use of accelerometers attached to 20-30-cm nails appears to be a low-cost, user-friendly method to locate and monitor the size of infestations of soil insects that tend to group together in clumps.

\section{Contributed Papers}

\section{8:50}

2aAB3. Two-tone suppression of the ultrasound induced startle response in a cricket. Hamilton E. Farris and Ronald R. Hoy (Sec. Neurobiology and Behavior, Mudd Hall, Cornell Univ., Ithaca, NY 14850, hef1@cornell.edu)

For the ground cricket, Eunemobius carolinus, frequencies $>20 \mathrm{kHz}$ elicit an acoustic startle response during flight. No startle responses are elicited by single pulses of low-frequency stimuli. However, simultaneous presentation of a low-frequency signal with ultrasound can suppress the startle response. The effect of frequency on suppression was measured for seven frequencies $(3-9 \mathrm{kHz})$ at four intensities of the startle stimulus (40 $\mathrm{kHz}): 5,8,10$, and $12 \mathrm{~dB}$ above startle threshold. Suppression was tuned to frequencies near $5 \mathrm{kHz}$. This effect of frequency on suppression diminished slightly at the higher ultrasound intensities, however. Because suppressor growth (change in suppression $\mathrm{dB}$ versus change in $40-\mathrm{kHz} \mathrm{dB}$ ) was $>1$ for $5-7 \mathrm{kHz}$ and $>1$ for 3 to 4,8 to $9 \mathrm{kHz}$, equal suppression curves flattened at the highest ultrasound levels. Startle threshold de- 
creases with increases in ultrasound duration, and temporal integration time constants were estimated to be $32 \mathrm{~ms}$. In nonsimultaneous presentations, however, no suppression occurs when the suppressing tone is $>2$ $\mathrm{ms}$ after and $>5 \mathrm{~ms}$ before the ultrasound stimulus. These times suggest that across-channel integration time is shorter than within-channel integration. [Supported by NIMH.]

\section{9:10}

2aAB4. Synchrony in cricket calling songs: Models of coupled biological oscillators. Timothy Forrest (Dept. of Biol., Univ. of North Carolina at Asheville, Asheville, NC 28804), Joel Ariaratnam, and Steve Strogatz (Cornell Univ., Ithaca, NY 14853)

Snowy tree crickets produce a rhythmic acoustic signal composed of short chirps. The chirp rhythms are very regular and dependent on temperature. When signaling in groups, males synchronize their chirps. The rhythmic signals of these tree crickets were used as a model of a "biological oscillator." The phase responses of males to single probe stimuli similar to the male calling song were measured. The phase response curves (PRC) of males showed both strong (type 0) and weak (type 1) resetting. PRCs differed depending on amplitude, duration, and frequency of the resetting stimuli. In other experiments, males could adjust their natural periods by as much as $15 \%$ when phase-locking (1:1) to an entrainment stimulus. Signaling behavior of males was compared with a simple model of coupled oscillators. The phase resetting was not consistent with the simplest resetting model, in which the male's phase shift is completed after one cycle of the chirp period. In addition, a simple iteration of the PRC could not simulate the phase-locking observed in the entrainment experiments indicating that the acoustic coupling between males differs from a simple resetting model.

\section{9:30-9:45 Break}

\section{$9: 45$}

2aAB5. Functional anatomy of the middle ear of insectivores. Matthew J. Mason (Dept. of Zoology, Univ. of Cambridge, Downing St., Cambridge CB2 3EJ, UK)

An electrical model has been constructed of the middle ear of land mammals [Hemilä et al., Hearing Res. 85, 31-44 (1995)]. This model can roughly predict the nature of air hearing in an animal, given the values of certain anatomical parameters for that animal. In the present study, light microscopy was used to investigate the anatomy of the middle ears of some representative members of the Insectivora. The nature of air hearing in these mammals was predicted, using an augmented version of the model. The model predicts that within the family Chrysochloridae (golden moles) there are genera (e.g., Chrysochloris and Chrysospalax) that have ears which appear to be very poorly adapted to the impedance-matching function required for efficient air hearing. The structure of the relatively enormous mallei of golden moles in these genera suggests that their ossicles are primarily adapted towards transmission of gound vibrations. A mechanism of ossicular inertia has been proposed. The electrical model predictions imply that subterranean mammals may be preadapted to evolve a more efficient mechanism for bone conduction of ground vibrations. Certain unusual features of the middle ear apparatus of other subterranean mammals may be interpreted in this light. [Work supported by B.B.S.R.C.]

\section{0:05}

2aAB6. Acoustics of the avian vocal tract. Neville Fletcher (Res. School of Physical Sci., Australian Natl. Univ., Canberra 0200, Australia, neville.fletcher@anu.edu.au) and Alex Tarnopolsky (Australian Defence Force Acad., Canberra 2600, Australia)

The avian vocal tract can appropriately be modeled on a source-filter basis, with the pressure-controlled syrinx valve providing the wideband harmonic source and the vocal tract the linear passive filter. This filter consists of four parts: the bronchial tubes above the syrinx valves, the trachea, the mouth with included tongue, and the beak. Each of these can be modeled and its behavior calculated quantitatively using standard methods [Fletcher, Acoustic Systems in Biology (Oxford U. P., New York, 1992)]. The long trachea defines the main resonance frequencies, upon which the bronchial resonances impose formant bands. The mouth, the cross section of which can be varied by changing the tongue position, influences particularly the frequencies of the first two tracheal resonances, but also the harmonicity of the upper system resonances. The beak behaves as a slotted tapered transmission duct, and contributes an endcorrection to the system, the magnitude of which is a strong function of both beak gape and frequency. This paper reports the numerical results of such a theoretical analysis, compares these with experimental measurements upon appropriate model systems, and discusses the implications of the results for the understanding of avian vocalization.

\section{$10: 25$}

2aAB7. Bioacoustic correlates of female choice in European starlings. Timothy Q. Gentner and S. H. Hulse (Johns Hopkins Univ., Dept. of Psych., Baltimore, MD 21218)

The elaborate structure of male European starling song suggests that it is a trait under heavy sexual selection pressures, and the results of several studies in both the laboratory and the field have identified song as the only male characteristic correlated with female choice in this species. However, previous studies have failed to isolate the bioacoustic feature(s) of song that are important for mating decisions and preferences in female starlings. An operant apparatus that reinforces a natural approach response to potential nest sites with different male starling songs is used to demonstrate that female preference is positively correlated with song bout length but not repertoire size (two features that normally covary). Further, this preference for longer song bouts is made in such a way that the absolute length of a bout cannot be the feature upon which the preference is based. A method for quantifying the temporal structure and stereotypy in male starling songs is presented using information theory, and it is shown that these measures correlate very closely with different song bout lengths. The importance of song bout stereotypy for female preference is then tested using synthetic stimuli or operant apparatus. 


\title{
Session 2aAO
}

\section{Acoustical Oceanography and Underwater Acoustics: Surf Zone Oceanography and Acoustics I}

\author{
James F. Lynch, Chair \\ Department of Applied Ocean Physics and Engineering, Woods Hole Oceanographic Institute, 203 Bigelow Building, \\ Woods Hole, Massachusetts 02543 \\ Chair's Introduction-9:15 \\ Invited Papers \\ 9:20
}

2aAO1. The Scripps Pier Bubble Experiment of 1997 and overview of rip events and their effects on acoustics measurements. Jerald W. Caruthers, Paul A. Elmore, Stephen J. Stanic (Naval Res. Lab., Stennis Space Center, MS 39529), and Ralph R. Goodman (Appl. Res. Lab., Penn State Univ., State College, PA 16804)

An experiment to determine the dynamics, distributions, and acoustic effects of bubbles in shallow water just offshore from active surf was performed in the Spring of 1997. The region of interest was just North of the pier at the Scripps Institution of Oceanography. An area from a few tens of meters to a thousand meters from the beach was instrumented by several researchers. An effort was made to acquire a comprehensive dataset of coastal dynamics, bubble distributions, and acoustic propagation. A major emphasis was placed on a smaller region approximately $400 \mathrm{~m}^{2}$ in area and about $300 \mathrm{~m}$ offshore, where most of the instruments were clustered. The purpose of this paper is to provide an overview of the experiment, a summary of the runs made over the several days of experiments, and a context for the observed rip events based on an acoustic device known as the "Delta-Frame." Two rip events (Run 5, event starting about 14:48 PST 7Mar97 and Run 7, event starting about 15:38 PST 8Mar97) selected for coordinated analyses and presentations to follow will be highlighted. [Work supported by ONR.]

\section{9:40}

2aAO2. Vertical profiles and horizontal scales of bubble clouds in the surf zone as measured with a distributed array of upward-looking sonars. Peter H. Dahl (Appl. Phys. Lab., Univ. of Washington, Seattle, WA 98195), Jerald W. Caruthers, and Stephen J. Stanic (Naval Res. Lab., Ocean Acoust. Branch, Stennis Space Center, MS 39529)

A collaborative, multi-institute experiment was recently performed in the vicinity of the Scripps Pier, whose goal was the study of bubble-field generation, transport, and distribution, as influenced by surf zone conditions. The collaboration produced an ensemble of instrumentation at the site for measuring bubbles, ambient noise, temperature and salinity, currents, and surface waves. This paper emphasizes data gathered by APL-UW using a set of four upward-looking transducers (frequency $240 \mathrm{kHz}$ ), which simultaneously measured vertical profiles of acoustic volume scattering from bubbles from four locations. The primary locus of measurement activity was defined by NRL-SSC's triangular frame (Delta Frame) and the four transducer locations for the APL-UW system bracketed this region. The transport of bubbles via rip currents emerged as a key feature surf zone bubble environment. Images of volumetric backscattering strength versus time and depth reveal episodic events (by way of increased scattering level) which last $\mathrm{O}(100) \mathrm{s}$ and can occur in intervals of $\mathrm{O}(10) \mathrm{min}$. Time lags for increased scattering at the four locations were consistent with a seaward flow of rip currents equal to $\sim 0.25 \mathrm{~m} / \mathrm{s}$. Other features of the data that complement the single-depth measurements made from the Delta Frame will be discussed. [Work supported by ONR.]

\section{Contributed Papers}

10:00

2aAO3. Bubble generation and dispersion in the surf zone. Svein Vagle, David Farmer (Inst. of Ocean Sci., 9860 West Saanich Rd., Sidney, BC V8L 4B2, Canada), and Grant Deane (Scripps Inst. of Oceanogr., La Jolla, CA 92093)

A combination of horizontal Doppler imaging, underwater microphotography, and in situ acoustical measurements are used to examine wave propagation, wave breaking, bubble cloud formation, and dispersion in the surf zone near Scripps Pier. Wave breaking is interpreted in terms of the evolution in wave properties between the end of the pier and a sensor tripod mounted in the surf zone. The position of the breaking zone is tracked from sharp discontinuities in the horizontal imaging sonar. Bubble size distribution is measured both photographically and with an acoustical resonator. From time to time the imaging Doppler sonar shows bubble clouds moving offshore from the surf. The observations provide a framework for model analysis of bubble generation, advection, and dispersion in the surf zone.
10:15

2aAO4. Bubbles and surf zone oceanography. Eric J. Terrill and W. Kendall Melville (Marine Physical Lab, Scripps Inst. of Oceanogr., 8605 La Jolla Shores Dr., M. S. 0230, La Jolla, CA 92093-0230)

Breaking waves in the surf zone entrain high-density clouds of bubbles which are subsequently transported offshore via rip currents and turbulent diffusion. The presence of the bubbles presents a complex problem for acousticians interested in the propagation of natural and man-made sound in this shallow water regime. To investigate the acoustical properties of this area, a multi-investigator experiment was performed near the Scripps Pier in March 1997. A broadband acoustic technique, developed at the Marine Physical Laboratory, was deployed in a four-channel configuration in water of 6-m depth. Each channel of the system allows the determination of the bubble size distributions at a discrete depth via inversion of the measured sound speed and attenuation. Analysis of the data shows high concentrations of bubbles advecting offshore via rip currents, resulting in acoustic attenuations ranging from $\mathrm{O}(1)-\mathrm{O}(100) \mathrm{dB} / \mathrm{m}$. Vertical gradients 
were observed in the bubble concentrations. Simultaneous measurements of the wind, waves, and currents provide a synoptic view of the overlying oceanography during the course of the experiment. Results of the experiment will be discussed in the context of the transport of surf-generated bubbles through the measurement site.

\section{0:30-10:45 Break}

\section{0:45}

2aAO5. Void fraction and bubble distributions near surf zones. Ming-Yang Su (Oceanogr. Div., Naval Res. Lab., Stennis Space Center, MS 39529)

Extensive measurements of void fraction and bubble size density due to wave breaking both inside and outside surf zones were made by the Naval Research Laboratory during two joint experiments sponsored by ONR: the Scripps Bubbles/Acoustics Experiment at Scripps Institution of Oceanography, March 1997, and the Sandy Duck '97 Experiment at the Army CERC Field Research Facility, Duck, NC, Sept.-Oct. 1997. Using a new technique that employs swing bar void fraction staffs anchored on the sand bottom, the new data show very strong tidal effects on the vertical distributions of void fraction. Data will also be presented on the temporal evolution of bubble size density (radius $=15-1200 \mu$ ) during local storms. Finally, some new results on direct correlation of acoustic noise intensity using a hydrophone with co-located void fraction measurement will be presented.

\section{1:00}

2aAO6. Tomographic reconstruction of evolving bubble fields in the Scripps Pier bubble experiment. Daniel Rouseff and Frank S. Henyey (Appl. Phys. Lab., College of Ocean and Fishery Sci., Univ. of Washington, Seattle, WA 98105, rouseff@apl.washington.edu)

The Delta Frame, designed and operated by NRL-SSC, was a major component of the 1997 Scripps Pier shallow water bubbles experiment. The frame supported two sources and eight receivers. Acoustic travel time and attenuation were measured at eight frequencies between 39 and 244 $\mathrm{kHz}$ over the resulting 16 intersecting ray paths. Paths ranged in length from 2.5 to $8.6 \mathrm{~m}$. For a given transmission sequence, the measurements at a particular frequency can be combined using tomography to yield a quantitative cross-sectional image of the ensonified region. By generating images for successive sequences, the temporal evolution of the medium can be visualized. A tomography algorithm [D. Rouseff and F. S. Henyey, J. Acoust. Soc. Am. 101, 3032 (1997)] is applied to the Delta Frame data. The images are produced for time periods during the experiment where the bubble field was probed by other independent measurements. Tomography results are compared to the other measurements. [Work supported by ONR.]

\section{1:15}

2aA07. Effects of bubbles on high-frequency sound propagation in very shallow water. Paul A. Elmore, Jerald W. Caruthers, Stephen J. Stanic (Naval Res. Lab., Stennis Space Center, MS 39529), and Ralph R. Goodman (Appl. Res. Lab., Penn State Univ., State College, PA 16804)

The results of an experiment performed off the research pier at the Scripps Institute of Oceanography that measured the acoustic effects of small bubbles in very shallow water $(6 \mathrm{~m})$ are discussed. The results presented are in coordination with researchers who conducted other measurements simultaneously. During the experiment, rip currents passed through a field of measurement instruments $300 \mathrm{~m}$ offshore. These rip currents were laden with bubbles created in the surf between the instruments and the shore. Pulse signals, between 39 and $244 \mathrm{kHz}$, were propagated to $10 \mathrm{~m}$ through the bubble clouds. The effects of these rip currents on the spatial distributions of the resulting acoustic attenuation are discussed. From the attenuation data, bubble populations are calculated by an iterative procedure based on the well-known resonant bubble approximation. [Work supported by the Office of Naval Research.]
11:30

2aAO8. A novel approach for obtaining bubble dissolution measurements at sea. Russell Costa (Naval Undersea Warfare Ctr., Newport, RI 02841), Ronald A. Roy (Boston Univ., Boston, MA 02215), Kerry W. Commander, Victor B. Johnson, and Andrew C. Colbert (Naval Surface Warfare Ctr., Panama City, FL 32407-7001)

A hybrid system for measuring the dissolution of gas bubbles in an oceanic environment was developed utilizing the Coastal Systems Station's Light Scattering Bubble Counter (LSBC). The LSBC independently measures flow velocity and bubble size, and is capable of distinguishing bubbles from particulate matter. In the hybrid system, the dynamic range of the LSBC measurements is $50 \mu \mathrm{m}$ to about $300 \mu \mathrm{m}$ in radius. The system is filled at depth ( 30 feet maximum without internal pressurization) and initially purged of all free gas. Pneumatically controlled valves are used to establish a closed recirculating system in which a flow can be generated in either direction using a large-volume peristaltic pump driven at low-volume velocity. Clear air is forced through a ceramic fritted disk to create the initial bubble population. This bubbly mixture is recirculated through the system at a nominal flow rate of $22 \mathrm{l} / \mathrm{min}$, which corresponds to a velocity of $30 \mathrm{~cm} / \mathrm{s}$ through the $\mathrm{LSBC}$ aperture. The bubble radii are continuously measured using the LSBC, resulting in time-dependent bubble size distributions. These distributions are used to estimate bubble dissolution rates for the size range indicated. [Work supported by ONR.]

\section{1:45}

2aA09. Analysis of free bubble cloud scattering data from the Lake Seneca experiment. J. Gregory McDaniel, Ronald A. Roy, Andrew D. Gephart (Dept. of Aerosp. and Mech. Eng., Boston Univ., Boston, MA 02215), and William M. Carey (MIT, Cambridge, MA 02139)

In the fall of 1991, Carey, Roy et al. conducted a series of experiments at the Seneca Lake test facility which measured the frequency-dependent scattering from submerged bubble clouds. Preliminary results reported by Roy et al. [J. Acoust. Soc. Am. 92, 2993-2995 (1992)] showed that certain features of the high-frequency backscatter data were consistent with single-bubble scattering while the low-frequency results were consistent with collective oscillations of the bubble cloud. A monopole resonance scattering peak was evident that scaled with the bubble cloud volume and the mixture sound speed. Additional analysis showed that the evolving target strength of the cloud as it rose through the insonating acoustic beam showed signs of an interference effect [Carey and Roy, Proc. 2nd Euro. Conf. on Underwater Acoust., European Commission, Luxembourg, 207 (1994)] which could explain the differences between calculated and measured scattering strengths. Advanced signal processing and modeling techniques have been developed to enable the removal of experimental artifacts caused by: (1) uncertainties in the range geometry, (2) coherent electrical noise in the processing electronics, and (3) acoustic reverberation within the test volume. The resulting corrected backscattering target strengths will be reported and discussed. [Work supported by ONR.]

\section{2:00}

2aAO10. Sound radiation by a bubble encountering a vortex ring. Ali R. Kolaini (Natl. Ctr. for Physical Acoust., Univ. of Mississippi, University, MS 38677, ali@sparc.ncpa.olemiss.edu)

The dynamics of a bubble and subsequent acoustic radiation interacting with a vortex ring created by an underwater nozzle are studied both theoretically and experimentally. The velocity field of the flow containing the bubble may be represented as a "basic flow" related to the vortex ring and a "secondary flow" related to the bubble motion [G. Chahine, 20th Symposium on Naval Hydrodynamics (1994)]. The "bubble flow" is assumed to be irrotational satisfying the Laplace equation. The vortex related velocities are given by the stream function and are taken into account by imposing "correct" kinematics and dynamic boundary conditions at the bubble surface. The interaction of the bubble with a vortex ring has been shown to cause severe deformation. Under special circumstances the deformed bubble may be at resonance with these vortex filaments and 
force it to radiate sound to the far field. The boundary integral technique (BIT) is employed to solve governing equations. The BIT is utilized to simulate the "bubble flow," its surface distortions and subsequent acoustic radiation. The critical parameters responsible for the acoustic re- excitation of the bubble encountering the vortex filaments are discussed in some details. Some conclusions are arrived at on the acoustical characteristics of the bubble encountering a fully developed turbulent flow field. [Work supported by ONR.]

TUESDAY MORNING, 23 JUNE 1998

GRAND BALLROOM A (S), 9:15 A.M. TO 12:05 P.M.

\title{
Session 2aBV
}

\section{Bioresponse to Vibration/Biomedical Ultrasound and Physical Acoustics: High Intensity Therapeutic Ultrasound I}

\author{
Pierre D. Mourad, Cochair \\ Applied Physics Laboratory, University of Washington, 1013 NE 40th Street, Seattle, Washington 98105 \\ Gail R. ter Haar, Cochair \\ Physics Department, Royal Marsden Hospital, Sutton, Surrey SM2 5PT, England
}

Chair's Introduction-9:15

Invited Papers

9:20

2aBV1. Biological effects of ultrasound. Wesley L. Nyborg (Phys. Dept., Univ. of Vermont, Burlington, VT 05405)

\begin{abstract}
Ultrasound has considerable potential as an agent for modifying cells and tissues. This comes partly from its capability to produce temperature elevation in desired spatial and temporal patterns. It also comes partly from its capability to produce cavitation in one or more of its diverse forms and, in addition, partly from the action of radiation forces, radiation torques, and acoustic streaming. In some situations, several mechanisms are involved and may interact with each other; for example, cavitation can produce heat in a medium, and the resulting temperature rise will affect the cavitation activity. Understanding these and other mechanisms has been, and promises to be, helpful in developing applications of ultrasound to therapy, surgery, and biotechnology, as well as in formulation of criteria for safety in medical procedures involving ultrasound. In this review, examples will be given to which the mechanisms are relevant, and recent developments will be discussed.
\end{abstract}

9:40

2aBV2. Therapeutic ultrasound. Lawrence A. Crum, Michael Bailey, Kirk Beach, Michael Caps, Stephen Carter, Wayne Chandler, Robin Cleveland, Scott Helton, Peter Kaczkowski, George Keilman, Roy Martin, Pierre Mourad, Ronald Roy, Udo Schmiedl, Brent Stewart, and Shahram Vaezy (Univ. of Washington, Seattle, WA 98105)

Although diagnostic ultrasound is now a multibillion dollar a year industry, the therapeutic applications of ultrasound were initially thought to have more promise. Indeed, the Fry brothers were performing brain surgery on human patients in the early 1950s, and with considerable success. However, therapeutic ultrasound has still not yet gained its promising potential. Recently, there has been an enormous resurgence of interest in this topic, and several major projects are underway at various facilities around the world. Our own substantial efforts in this area at the University of Washington are reviewed, with brief discussions of topics in lithotripsy, ultrasound-enhanced drug delivery, HIFU surgery, and acoustic hemostasis. [Work supported in part by DARPA and the NIH under Grants DK-43881 and R43HL57739.]

10:00

2aBV3. Acoustic liver cauterization: A potential tool for bloodless surgery. Roy Martin (Anesthesiology and Bioengineering, Box 356540, Univ. of Washington, Seattle, WA 98195, rmartin@u.washington.edu), Shahram Vaezy, Scott Hilton, Michael Caps, Peter Kaczkowski (Univ. of Washington, Seattle, WA 98195), George Keilman (Sonic Concepts, Woodinville, WA 98072), Steve Carter, Wayne Chandler, Pierre Mourad, Kirk Beach, and Lawrence Crum (Univ. of Washington, Seattle, WA 98195)

Liver surgery is hampered by the difficulty in controlling hemorrhage, particularly the slow oozing from injured hepatic surfaces. Conventional cauterizing fails in this situation. Bleeding from incisions made in animal livers using high-intensity focused ultrasound (HIFU) by volume cauterizing along the surface of the incision has been successfully stopped. The question arose: Could bleeding be avoided during surgery by precauterizing a thick surface around a region to be resectioned prior to the actual resectioning? The livers of anesthetized rabbits (4) and pigs (3) were treated in this manner under a protocol approved for animal studies at the University of Washington. Two HIFU transducers $(3.3$ and $3.5 \mathrm{MHz}$ focused at 3.5 and $5.5 \mathrm{~cm}$, respectively) were used for the treatment. 
Cauterized strips around a region appeared white. The tissue to be resectioned was removed by cutting along the distal border of the strips. No oozing from the liver parenchyma was observed. Bleeding occurred only in the larger vessels ( $>2 \mathrm{~mm}$ inside diameter) present in the cauterized region. Since these size vessels are easily ligated the method appears to be clinically feasible. Survival studies are needed to learn about the possibilities of rebleeding after abdominal closure or unknown healing difficulties.

\section{Contributed Papers}

\section{0:20}

2aBV4. High-intensity focused ultrasound transducer design for surgical and hemostatic applications. George W. Keilman (Sonic Concepts, 20018 163rd Ave. NE, Woodinville, WA 98072) and Peter J. Kaczkowski (Univ. of Washington, Seattle, WA 98105-6698)

A resurgence in interest in the application of high-intensity focused ultrasound (HIFU) to surgical problems is due in part to significant improvements in imaging modalities for targeting the delivery of ultrasound. The wide variety of potential applications under consideration necessitates a refinement of transducer design to accommodate often conflicting constraints. The need to deliver substantial power to a small target zone requires high focal gain to minimize collateral damage, and yet must be balanced with the need for small transducers in many procedures. In general, the choice of operating frequency, transducer aperture and focal depth, convenient acoustic coupling methods, waveform selection, and amplifier characteristics must all be adjusted to best fit the application. This presentation will discuss such tradeoffs in any HIFU transducer design, and will also address particular techniques for coupling and cooling transducers which deliver hundreds of watts of acoustic power. Transducer efficiencies of over $85 \%$ have been routinely achieved by judicious selection of matching layers and tuning networks, while maintaining over $50 \%$ relative bandwidth, for center frequencies spanning the range from 1 to 10 MHz. Transducer requirements for array-based systems, motivated by clinical needs, will also be discussed. [Work funded by DARPA/ONR.]

\section{0:35}

2aBV5. Acoustic field of a device for noninvasive pressure wave therapy in orthopedy. Eckard Steiger, Thomas Dreyer (Inst. für Hoechstfrequenztechnik und Elektronik/Akustik, Univ. Karlsruhe, D-76128 Karlsruhe, Germany, eckard.steiger@etec.uni-karlsruhe.de), and Juergen Mayer (Storz Medical AG, CH-8280 Kreuzlingen, Switzerland)

The Storz Minilith SL1 is a device specially designed for the treatment of orthopedical indications as tendinosis calcarea, tennis or golf elbow, heel spurs, or supra spinatus syndrome. A cylindrical electromagnetical source generates an intense ultrasonic pulse, which is focused by means of a parabolical reflector. Measurements in the focal region are performed in degassed water using a fiber-optic probe hydrophone. The whole process of pulse radiation from the source over nonlinear propagation including reflection to the focusing is simulated too. The underlying full wave model is based on a specially developed finite difference in time domain method. Excellent agreements of measured and computed pressure wave profiles in the focal region validate the model. The generation of shock waves in dependence on the energy setting of the therapy device is studied. Further investigations are made to consider the interaction of the SL1-pulse with human tissue structures as they occur in medical applications.

\section{0:50}

2aBV6. Ex vivo studies of the effect of high-intensity focused ultrasound on whole human blood. Sandra Poliachik, Susanah Bloch, Michael Bailey, Peter Kaczkowski, Robin Cleveland, Pierre D. Mourad, Tyrone Porter, Lawrence A. Crum (Univ. of Washington, Appl. Phys. Lab, 1013 NE 40th St., Seattle, WA 98105, pierre@apl.washington.edu), Wayne L. Chandler (Univ. of Washington, Lab. Medicine, UW Medical Ctr.), and George W. Keilman (Sonic Concepts, Woodinville, WA 98072)

A surgical application of high-intensity focused ultrasound (HIFU) is hemostatis. In this application, both tissue and blood will be subjected to HIFU. What will happen to blood? The intent of this study is to explore via experiment some of the bioeffects of HIFU on whole human blood. A $1.1-\mathrm{MHz}$ piezoceramic source with a $60-\mathrm{mm}$ radius of curvature and $100-\mathrm{mm}$ aperture was used. Special acoustically transparent containers were made of handmade, low-density polyethylene tubing. Phenomena studied included hemolysis, morphologic changes in erythrocytes, white blood cell differential, and platelet activation, all as a function of acoustic intensity and dosage. Intensities up to $6000 \mathrm{~W} / \mathrm{cm}^{2}$ were used in $\mathrm{cw}$ pulses of one second. The bioeffects noted above were correlated with temperature and a passive acoustic measure of bubble activity within the blood created by the ultrasound. Under the acoustic conditions considered for this work, nonthermal acoustic effects were most strongly associated with bioeffects. [Work sponsored by DARPA.]

\section{Invited Papers}

\section{1:05}

2aBV7. Shaping of focused ultrasonic beams to expedite thermal necrosis in tumor therapy. Frederic L. Lizzi (Riverside Res. Inst., 330 West 42nd St., New York, NY 10036, lizzi@ rrinyc.org)

Ultrasonic tumor therapy employing focused beams shows great promise for treating tumors by producing spatial matrices of thermal-necrosis lesions. A practical problem involves the long time required to treat extended tumor volumes. To treat ocular tumors, our laboratories are investigating beams with asymmetric shapes to expedite the production of lesion matrices. The beams are generated using rectangular strip electrodes plated on spherical-cap ceramic transducers. The phase and amplitude of the strip excitation voltages are controlled to launch beams whose focal-zone patterns consist of a sequence of lobes confined within elliptical, rather than circular, cross sections. A simple theoretical model has shown that thermal diffusion between these lobes can produce lesions with elliptical cross sections; lesion matrices would be readily produced by translating such transducers along the short axis of the lesion cross section, thereby causing necrosis in broad regions. The concept has been verified in in-vitro experiments (in liver and the ocular lens) and in in-vivo exposures of human tumor explants in nude mice. [Work supported by NIH Grant EY10369.]

\section{1:25}

2aBV8. An image-guided ultrasound phased array system for noninvasive surgery. Emad S. Ebbini, Philip D. VanBaren, and Claudio Simon (Dept. of EECS, Univ. of Michigan, Ann Arbor, MI 48109-2122)

An integrated image-guided therapeutic phased array system for noninvasive surgical applications is being developed. The therapeutic array utilizes piezocomposite transducer technology and operates (therapeutically) at 1 and $2 \mathrm{MHz}$. It has 64 elements on a spherical shell with a geometric center at $100 \mathrm{~mm}$ from its apex. The array was shown to be capable of producing well-defined thermal lesions in tissue media at depths from 40 to $60 \mathrm{~mm}$ and to scan therapeutic foci up to $\pm 15 \mathrm{~mm}$ from its geometric center. 
Image guidance is provided by a modified diagnostic ultrasound scanner which, in addition to providing standard B-scan images of the target region, provides real-time images of the temperature rise due to the therapeutic beam. The temperature information is obtained using a correlation based algorithm for echo displacement estimation, which can be directly related to local variation in tissue temperature due to the therapeutic beam. A complete description of the system will be given along with illustrative examples of image-guided tissue ablation and temperature estimation and control. In addition, experimental data demonstrating the use of the therapeutic array in an imaging mode will be presented. [Work funded by NIH Grant CA66602.]

\title{
11:45
}

2aBV9. MRI monitoring and control of focused ultrasound surgery. Kullervo Hynynen, Nathan McDannold, Todd Fjield, and Douglas Daum (Div. of MRI, Dept. of Radiology, Brigham and Women's Hospital, Harvard Med. School, 221 Longwood Ave., Boston, MA 02115)

The clinical and animal tests of focused ultrasound surgery have shown significant variation between the effectiveness of the same power sonications. Therefore, it has become important to seek methods to monitor the in vivo ultrasound exposures. One of the most promising methods is the use of magnetic resonance imaging (MRI) to detect the temperature elevation. In this paper the calibration and accuracy of MRI thermometry will be presented. The in vivo mapped temperature distributions allow the thermal exposure of the tissue to be estimated. These estimates have been compared with the tissue coagulation produced in muscle and brain tissues in vivo. The results show that the lesion boundary is predicted with the resolution of the MRI voxels. This has significant clinical value since the MRI thermometry can be used to control the treatment on-line. These experiments allowed comparison of the accuracy of the theoretical models for predicting the temperature elevation and tissue necrosis in vivo. Similarly, the MRI thermometry was used to investigate the temperature buildup during multiple sonications and evaluate the performance of new applicators such as phased arrays in vivo.

TUESDAY MORNING, 23 JUNE 1998

EAST BALLROOM A (S), 9:15 TO 11:45 A.M.

\section{Session 2aEA}

\author{
Engineering Acoustics: Ultrasonic Transducers for Applications in Air \\ Dehua Huang, Chair \\ Arichell Technologies, Inc., Border Street, Newton, Massachusetts 02165
}

Chair's Introduction-9:15

Invited Papers

9:20

2aEA1. Influence of air loading and dissipation on plate transducer amplitude. Arthur Ballato (U.S. Army Commun.-Electron. Command, Fort Monmouth, NJ 07703-5201, a.ballato@ieee.org)

Air-loaded plate transducers driven piezoelectrically are used in a variety of modern applications such as optical modulators, microactuators, and certain types of resonant acoustic sensors and frequency control elements. For these uses it is necessary or desirable to know the maximum mechanical amplitudes obtainable, and how these are limited by the various internal dissipation mechanisms and the loading of the ambient fluid. Internal conduction, dielectric, and acoustic viscosity losses are incorporated, and, by introducing of a complex piezoelectric coupling coefficient, changes in critical frequencies and amplitudes are traced with variations in type and size of dissipation mechanism, and the ambient loading. The analysis also shows that the unavoidable interfacial stresses existing between the crystal plate surfaces and the exciting electrodes can become exceedingly large at the frequencies commonly used for frequency control of cellular communications. Because the various devices covered usually form components of an overall circuit design, a number of alternative equivalent electrical circuits are also provided whereby the physics of these structures may be rendered and readily interpreted in network terms.

9:45

2aEA2. Absolute amplitude measurements using a capacitive receiver. M. A. Breazeale (Univ. of Mississippi, Natl. Ctr. for Physical Acoust., University, MS 38677)

Absolute acoustical amplitudes can be measured in solids with a capacitive receiver. Amplitudes as small as $10^{-4} \AA=10^{-14} \mathrm{M}$ have been measured. A system is described with dimensions used for frequencies between 5 and $60 \mathrm{MHz}$. Two special innovations are described: low-temperature measurements which require adjustment of the spacing of the capacitor and high-sensitivity measurements (amplitudes smaller than $10^{-14} \mathrm{M}$ ) which are accomplished by using a dielectric to prevent electrical breakdown. 
2aEA3. Silicon micromachined ultrasonic transducers. B. T. Khuri-Yakub (Edward L. Ginzton Lab., Stanford Univ., Stanford, CA 94304-4085, khuri-ya@ee.stanford.edu)

This paper presents a novel type of ultrasonic transducer that is made by silicon micromachining. Transducers for both air-borne and immersion applications are made from parallel plate capacitors whose dimensions are controlled through traditional integrated circuit manufacturing methods. Typical dimensions of the capacitors are: gap $=1$ micron (vacuum or air), membrane thickness $=1$ micron (silicon nitride or polysilicon), and diameter $=50$ microns. A large number of small elements are connected in parallel to make a transducer. Transducers for air-borne ultrasound applications have been operated in the frequency range of $1-11 \mathrm{MHz}$, while immersion transducers have been operated in the frequency range of 1-20 MHz. Theoretical modeling calculations of the transducers and their electrical input impedances will be presented. The theory predicts a dynamic range of over $140 \mathrm{~dB}$ for both air and immersion applications. Different processing methods for fabricating the transducers and the merits of the different approaches will be presented. Finally, the results of transducer operation both in air and water with over a 100-dB dynamic range will be presented.

\section{$10: 35$}

2aEA4. Air transducers with high acoustic impedance. Lawrence C. Lynnworth (Panametrics, Inc., 221 Crescent St., Waltham, MA 02154, lynnworthl@panametrics.com)

High- $Z$ air transducers evolved from several industrial transducers. These predecessors include (a) acoustic emission and anglebeam NDT contact transducers; (b) flowmeter transducers for high-pressure methane, hot refinery gases, and corrosive flare gases; and (c) transducer arrays for monitoring hot corrosive gases flowing at Mach 0.1 in smokestacks of $\phi 3-13 \mathrm{~m}$. This peculiar ancestry accounts for their unusual high-acoustic-impedance construction. By not employing low- $Z$ air-backed radiating membranes, transducer bandwidth, response time, and sensitivity are sacrificed. The solid, robust construction, however, offers some compensating features: tolerance to wide ranges in pressure and temperature, including rapid rates of change (thermal shock, depressurization); corrosion resistance; operable with standard lab equipment, without bias voltage; mounting options where the transducer forms part of the pressure boundary, or where it is outside the pressure boundary. Clamp-on air flow applications at one bar include small wind tunnels and plastic pipes. Acoustically isolated pairs measure secondary flow components (crossflow, circulation) in a plane perpendicular to the pipe axis. Flow applications include gases such as air, mild steam (pending) or other hot, pressurized, or corrosive fluids. Air-coupled measurements of transmission characteristics of wood or other low-density solids, and air-ranging, are readily demonstrated with the low- $Z$ transducers.

\section{Contributed Papers}

\section{1:00}

2aEA5. Ultrasonic capacitance transducers for flow and temperature tomography in air. David A. Hutchins (Dept. of Eng., Univ. of Warwick, Coventry CV4 7AL, UK, dah@eng. warwick.ac.uk), William M. D. Wright (Univ. College Cork, Cork, Ireland), David W. Schindel (N.R.C., Ottawa, ON K1A 0R6, Canada), and Peter W. Carpenter (Univ. of Warwick, Coventry CV4 7AL, UK)

Capacitance transducers have been constructed that can operate at frequencies of up to $2 \mathrm{MHz}$ in air, over a wide bandwidth, and with good efficiency. These have been used to propagate signals in air across regions containing variations in air flow and temperature. A source and receiver pair were scanned along a set of projections under computer control, allowing various ray paths across a chosen cross section to be selected. For each location, the received waveform was digitized and recorded, and the propagation time determined from cross-correlation techniques. The data were then used to form tomographic images, which corresponded to variations in either temperature or flow speed, both of which affect the local acoustic velocity. In one experiment, an air-jet was imaged at various distances from the nozzle. In another, the temperature variations in air above a hot metal tip were determined. Images will be shown for both sets of experiments, and the usefulness of the technique discussed.

\section{1:15}

2aEA6. Ultrasonic air-coupled capacitance transducers using thin mica films. Paul Ingleby (Dept. of Eng., Univ. of Warwick, Coventry CV4 7AL, UK, es2041@eng.warwick.ac.uk), David A. Hutchins, Andrew G. Bashford (Univ. of Warwick, Coventry CV4 7AL, UK), and David W. Schindel (N.R.C., Ottawa, ON K1A 0R6, Canada)

Micromachined air-coupled capacitance transducers have been manufactured using anisotropically etched silicon backplates and commercially available mica films. Devices developed using $20-\mu \mathrm{m}$-thick insulating layers have yielded frequencies of up to $400 \mathrm{kHz}$. However, new devices using mica with a thickness of approximately $4 \mu \mathrm{m}$ will deliver characteristics similar to existing transducers employing polymeric dielectric films. This will result in wideband behavior and bandwidths extending from approximately $100 \mathrm{kHz}$ to $2 \mathrm{MHz}$. Mica-based devices have several advantages over capacitance transducers with polymer films due to the robust physical characteristics exhibited by this type of material. Improved thermal characteristics and lower porosity allow this type of device to be employed in a variety of conditions in which the performance of the polymer films would be impaired. Results of experiments performed in air at a range of temperatures from ambient to $400{ }^{\circ} \mathrm{C}$ will be presented. This will demonstrate the versatility of such devices in terms of both sensitivity and bandwidth

\section{$11: 30$}

2aEA7. Recent developments in air-coupled ultrasonic capacitive transducers for NDE. Craig S. McIntyre, David A. Hutchins (Dept. of Eng., Univ. of Warwick, Coventry CV4 7AL, UK, esrna@eng.warwick.ac.uk), and David W. Schindel (Inst. for Aerosp. Res., Natl. Res. Council, Ottawa, ON, Canada)

A new design of an air-coupled capacitive ultrasonic transducer has been developed that could have potential for use in NDE experiments, particularly when the pulse-echo method is employed. The design is based on a standard capacitive transducer, except the newly developed device has two concentric, backplate elements with a common membrane. Each of the elements is electrically isolated from the other, allowing one to be used as a source of ultrasound, while the other is used as a detector. This yields a device containing both a source and receiver of ultrasound within the same structure. Experiments have shown that the device performance depends on which of the elements is selected to be source and receiver, size of the bias voltage employed, surface of the backplates, and membrane material utilized. Initial studies have shown that the device is capable of detecting the front face echo from various material samples, and these results are contained within this paper. There has also been some investigation of the signal quality when capacitive devices have been placed within an enviroment containing a range of pressures above standard atmospheric pressure. Applications of this work include measurements in high-pressure gas pipelines. 


\title{
Session 2aMU
}

\section{Musical Acoustics: Physics and Materials of Musical Instruments I}

\author{
Shigeru Yoshikawa, Chair \\ Department of Acoustical Design, Kyushu Institute of Design, 4-9-1 Shiobara, Minami-Ku, Fukuoka, 815 Japan
}

\section{Invited Papers}

\section{$7: 45$}

2aMU1. Fluid dynamic aspects of human voice and brass instruments: Implications for sound synthesis. Pelorson Xavier (Institut de la Commun. Parlee, ESA CNRS 5009, 46 av. F. Viallet F-38031 Grenoble, France), Msallam Regis (Institut de Recherche et de Coordination Acoustique Musique, F-75004 Paris, France), Gilbert Joel (Institut d'Acoustique et de Mecanique, F-72017 Le Mans Cedex, France), and Hirschberg Avraham (Tech. Univ. of Eindhoven, NL-5600 MB Eindhoven, The Netherlands)

The challenge of physical models of musical instruments is to provide a global description of a highly complex instrument which has to be accurate but simple enough to be numerically implemented. For this purpose, a logical approach is to extract, from a theoretical or experimental study, what are the most important phenomenon involved which are thus to be taken into account. Two examples of such an approach are given by the physical models of human voice and brass instruments presented here. Although both instruments share in common the same basic principle, a self-oscillating source coupled with a resonator, crucial physical aspects are quite different. It is shown that, for brass instruments, the lips' self-sustained oscillations are mainly driven by the acoustical coupling, therefore the simpliest one degree of freedom model for the lips is sufficient. In the case of voicing, the acoustical feedback, although effective, is not sufficient to maintain self-sustained oscillations. A more elaborated vocal-folds model is needed. Regardless of the sound source, the description of the resonator is also discussed. While, for voice simulations, significant improvement can be gained using a two-dimensional linear theory, nonlinear propagation is sometimes to be taken into account for brass instruments.

8:10

2aMU2. The effect of wall materials on the timbre of brass instruments. Robert W. Pyle, Jr. (11 Holworthy Pl., Cambridge, MA 02138-4509)

It has long been thought by players and instrument makers that the material from which a brass instrument is made influences the tone quality and playing characteristics of the instrument. For instance, the brochure of a maker of custom trombones describes bells made of three alloys: yellow brass $(70 \% \mathrm{Cu}, 30 \% \mathrm{Zn})$, having the "clearest sound, sharpest articulation," gold brass $(85 \% \mathrm{Cu}, 15 \%$ $\mathrm{Zn})$, with "warm sound, rounded articulation," and red brass ( $90 \% \mathrm{Cu}, 10 \% \mathrm{Zn})$, with "warmer sound, covered articulation." The bells are made in three metal thicknesses: light weight, with "flexible tone, rapid response," standard weight, with "balanced tone, even response," and heavy weight, with "firm tone, stable response." Most players agree with these statements. This model of trombone is made so that bell sections can be interchanged while leaving the remainder of the instrument intact. The present paper reports the results of measurements under playing conditions on several different bells. Comparisons of steady-state behavior are made via the transfer function between sound pressure in the mouthpiece cup and an on-axis point outside the bell. An attempt will also be made at the rather more difficult task of characterizing the attack transient.

\section{8:35}

2aMU3. The effect of wall properties on the timbre of organ pipes. Judit Angster (Fraunhofer-Institut fur Bauphysik, Nobelstr. 12, D-70563 Stuttgart, Germany, angster@ibp.fhg.de), Gyvrgy Paal, Walter Garen (Fachhochschule Ostfriesland, Constantiaplatz 4, D-26723 Emden, Germany), and Andras Miklss (Fraunhofer-Institut fur Bauphysik, D-70563 Stuttgart, Germany)

The influence of the wall vibrations on the timbre of flue organ pipes is one of the most disputed questions of organ building. Wall vibrations of metal and wooden labial pipes have been investigated by using miniature piezoelectric accelerometers and optical noncontact vibrometers. Both free and forced vibrations have been studied. Two sets of diapason pipes that were built with different wall properties but identical dimensions have been voiced to very similar speech and timbre, then vibration and sound spectra and attack transients were recorded. While large differences have been found in vibration spectra, hardly any change in the sound signal was observed. Similar investigations have been performed on wooden pipes. In this case the voicer "tuned" the side wall resonances in order to make the sound richer in overtones. The effect of this "tuning"' on the vibration and sound spectra has been investigated. The "tuning" resulted in a significant increase of the vibration amplitude, while the radiated sound-pressure level decreased and the overtones became relatively stronger.

\section{9:00}

2aMU4. Acoustically driven sinuous instability of a planar air jet. A. W. Nolle (Dept. of Phys., Univ. of Texas, Austin, TX 78712-1081)

The sinuous instability wave of a planar air jet is excited by localized acoustic flow across the nozzle. Phase velocity and the growth exponent are found from synchronous hot-wire measurements made beyond the excited region, where the profile is approximately sech squared. In the observed range of scaled radian frequency, 0.02-1.33 (the stability limit), results agree with real- 
frequency (spatially growing) analysis but not with complex-frequency (temporally growing) analysis, which predicts smaller phase velocity at low frequencies. In further tests, the acoustic driving signal is independent of downstream distance, as in an organ pipe. The jet deflection is then the sum of acoustic convection and of the instability wave, summing to zero at the nozzle, as proposed by Fletcher, Elder, and others. The instability-wave theory applies to linear behavior in the inviscid limit and therefore to a hypothetical nonspreading jet. The local velocity profile width must be considered in relating to a physical jet. In a flue organ pipe oscillating at equilibrium amplitude the jet deflection at the lip exceeds the range of validity. Direct sound generation by the jet is investigated briefly.

\section{9:25-9:35 Break}

9:35

2aMU5. Jet wave amplification in organ pipes. Shigeru Yoshikawa (Dept. of Acoust. Des., Kyushu Inst. of Design, 4-9-1 Shiobara, Minami-Ku, Fukuoka, 815 Japan)

The amplification factor of an air jet waving over the mouth is estimated from flow visualization of the smoked jet, where the velocity ranges from $7-33 \mathrm{~m} / \mathrm{s}$, the Reynolds number from 1000-5000, and the sounding frequency from $130-580 \mathrm{~Hz}$. The jet length is 15.8 and $10.2 \mathrm{~mm}$ in two models, while the jet thickness is $2.2 \mathrm{~mm}$ in both. The estimation is carried out by comparing the visualized envelope of jet waves with a simplified theoretical one. The envelope is visualized by the OR summation of the digitally memorized frames showing the instantaneous deflection taken with a speed of 1440 frames per second. Furthermore, the strength of mouth field defined as the acoustic displacement amplitude is derived from the envelope-based estimation. A hot-wire anemometer is then applied to measure this mouth-field strength, whose value shows a good agreement with the estimated result. The amplification factor of organ jets was estimated as 0.26 to $0.18(1 / \mathrm{mm})$; the mouth-field strength as 0.5 to $1.5 \mathrm{~mm}$. A nondimensionalized representation of the relation between the amplification factor and the blowing velocity in our organ-pipe jets roughly agrees with the theoretical curve based on the linearized spatial analysis of jet instability.

\section{Contributed Papers}

10:00

2aMU6. Interpretation of shear material properties of vocal fold mucosal tissues with Fung's quasilinear viscoelastic theory. Roger W. Chan and Ingo R. Titze (Natl. Ctr. for Voice and Speech, Dept. of Speech Pathol. and Audiol., The Univ. of Iowa, Iowa City, IA 52242, roger-chan@uiowa.edu)

Fung's quasilinear viscoelastic theory (QLV) [Y. C. Fung, Biomechanics (Springer-Verlag, New York, 1993), pp. 277-292] has been successfully applied to model the time- and history-dependent properties of a variety of biological soft tissues in the passive state. In particular, the hysteresis or damping curves of many soft tissues remain relatively insensitive to the rate of deformation (strain rate or frequency) across several decades of variation. This insensitivity to frequency cannot readily be accounted for by traditional discrete models of linear viscoelasticity. Rather, Fung's QLV provides a mathematically powerful framework in which a continuous stress relaxation spectrum can be used to model the relatively flat damping characteristics. The shear properties of human vocal fold mucosal tissues were measured with a rotational rheometer. Data on complex dynamic shear modulus, complex dynamic viscosity, and damping ratio as functions of frequency were obtained. It was found that vocal fold mucosal tissues show the shear-thinning behavior and have a relatively flat damping curve typical of biological soft tissues. Theoretical predictions based on the QLV matched well with the empirical data. The results suggest that the vocal fold mucosa can be modeled by the QLV under small-amplitude oscillation conditions. [Work supported by NIH Grant No. P60 DC00976.]

\section{0:15}

2aMU7. Theoretical and experimental investigation of the air-driven free reed. James P. Cottingham (Phys. Dept., Coe College, Cedar Rapids, IA 52402)

The free reed is the excitation mechanism for several families of instruments, including the reed organ and harmonium, the harmonica, the accordion and concertina, and the Asian free reed mouth organs. While most studies of reed behavior have concentrated on reeds which are either inward-striking (closing) and outward-striking (opening), free reeds do not fit neatly into either category and can exhibit behavior characteristic of either opening or closing reeds, depending on certain details of the reed configuration. Some characteristics of free reed behavior, in particular the relation between frequency and blowing pressure, have been modeled by adapting Fletcher's analysis of musical instruments driven by a reed mechanism [N. H. Fletcher, Acustica 43, 63-72 (1979)]. In addition, a variety of measurements of reed motion have been made, including experimental determination of measurements of reed position and phase using proximity sensors. These are compared with observations of the nearfield sound as well as with predictions based on theoretical considerations.

10:30

2aMU8. Material and obsolescence on flute tone quality. Alessandro Cocchi and Lamberto Tronchin (DIENCA - CIARM Univ. of Bologna, Viale Risorgimento, 240136 Bologna, Italy, tronchin@ciarm.ing.unibo.it)

The rule of material and obsolescence on flute tone quality has been discussed for a long time among musicians and flute makers. Mozart himself made use of crystal flutes in some of his works (Rondo', K617, Adagio, K356). From an acoustical point of view, since Boehm's work of 1832 , the effect of material on flute tone quality has not been investigated, until researches of recent years [Coltman (1971); Chapuis (1991)]. In this work the research has been carried out measuring impulse responses inside the tube of the flute. Two different flutes have been utilized, i.e., a lightalloy flute and a silver flute. The MLS sequences, with homemade software, have been utilized, gathering impulse responses in two different positions in the tube, in order to verify the variation of sound field. From the impulse responses a frequency analysis has been obtained, which pointed out that the silver flute contains more high frequency and shorter transient than the light-alloy flute. Starting from the impulse responses, sound example of difference in sound quality has finally been obtained. 
Noise and Architectural Acoustics: Acoustics of Buildings

\author{
Mei W. Wu, Cochair \\ Colin G. Gordon and Associates, 411 Borel Avenue, Suite 425, San Mateo, California 94402 \\ Colin G. Gordon, Cochair \\ Colin G. Gordon and Associates, 411 Borel Avenue, Suite 425, San Mateo, California 94402
}

Chair's Introduction-8:25

\title{
Contributed Papers
}

\section{$8: 30$}

2aNSa1. Active attenuation of fan noise in an air-conditioning duct. Ondrej Jiricek and Petr Konicek (CTU-FEE, Dept. of Phys., 16627 Prague, Czech Republic)

In this paper, the results of an investigation on using ANC systems in a duct will be presented. This system was used to reduce the noise of the axial-flow fan used as the noise source. The system was based on the adaptive digital signal processing controller. The attenuation of noise at low frequencies was achieved. This research represents a part of the project focused on the improvement of working conditions in operating theaters.

\section{8:50}

2aNSa2. Studies on a new type of silencer for air ducts. Fathy B. Shenoda (Dept. of Acoust., Natl. Inst. for Standards, Giza-Egypt, P.O. Box 136 Giza, Code No. 12211) and Reda N. Haroun (Dept. of Acoust., Natl. Inst. for Standards, Giza, Egypt)

The research work deals with a study of a specific type of silencer previously designed and tested by Shenoda. It consists of a rectangular duct, which is treated at its side walls with a gradually variable flow resistance. It was realized by increasing the perforated area along the duct wall combined with an homogeneous sound absorbing material. Based on the assumptions that only plane waves propagate in the duct, ignoring the effect of the mean flow velocity (very low) and applying linear acoustic equations, the sound propagation in such a silencer was studied. Therefore the wave impedance, the sound reflection coefficient at the input of the silencer, and the transmission loss introduced by it were calculated, measured, and compared with a silencer of comparable dimensions which is treated with a constant flow resistance along its length. To optimize the design different configurations were theoretically studied, computed, and compared. An important result: The silencer performance depends on a unique design parameter, namely, the silencer characteristic area. The methods of realizing a variable wall flow resistance were introduced. The effect of using different kinds of sound absorbing materials and changing the graduality of the variable flow resistance were measured.

\section{9:10}

2aNSa3. Noise of cleanroom recirculation systems. Mei Q. Wu and Colin G. Gordon (Colin Gordon \& Assoc., 411 Borel Ave., Ste. 425, San Mateo, CA 94402)

Recirculation air systems are used by the microelectronics industry as the primary source of unidirectional air flow in cleanrooms. The commonly used recirculation systems include: (1) packaged air handlers, which include centrifugal or plug (plenum) fans, generally located at fan deck level above the cleanroom; (2) fan-tower recirculation systems, consisting, most often, of vertically mounted vaneaxial fans arranged along the cleanroom perimeter; (3) individual fan-filter units, in which the
HEPA or ULPA filters are combined with small direct drive blowers, supported by the ceiling suspension system; and (4) minienvironments, which create superclean conditions around individual tools within a less clean "ballroom." In this paper, the commonly used recirculation systems will be described, typical sound power levels and spectrum characteristics of the systems will be presented, the natural noise attenuation along the air flow paths will be listed, the theoretical models for predicting noise levels in a cleanroom will be introduced, and noise mitigation means which can be integrated into different systems will be discussed. Also presented will be the typical noise levels measured in operating cleanrooms.

$$
\text { 9:30-9:45 Break }
$$

\section{9:45}

2aNSa4. Noise and vibration characteristics of cleanroom fan filter units. Mei Q. Wu and Colin G. Gordon (Colin Gordon \& Assoc., 411 Borel Ave., Ste. 425, San Mateo, CA 94402)

Fan filter units (FFU's) are used increasingly by the microelectronics industry to provide recirculation air in cleanrooms. Such units usually consist of one or more direct-drive blowers located in a small discharge plenum and a HEPA or ULPA filter. There may be several hundred FFU's in a large cleanroom. The noise and vibration generated by FFU's differ greatly between manufacturers. Since it is difficult to apply external noise and vibration mitigation, it is important to select an FFU model with appropriate noise and vibration levels, so that a cleanroom served by these units will meet the prespecified noise and vibration criteria. Unfortunately, FFU manufacturers usually do not provide enough information for predicting the FFU generated sound-pressure spectrum or vibration in a cleanroom. In this paper, simple noise and vibration measurement methods will be described, which can be used as a basis for specifying FFU performance without special testing facilities. Measured data will be presented for a number of units. Procedures will be demonstrated to predict the performance of a cleanroom which uses FFU's as the primary source of recirculation air.

\section{0:05}

2aNSa5. Design and acoustic characteristics of an anechoic chamber with high-power heating or cooling and sound field free transform system. Weicheng Yang and Jiahua Yang (Res. Inst. of Household Elec. Appliance of China, No. 6 Yuetan Beixiaojie, Beijing 100037, PROC)

In this paper, the design and the acoustic characteristics of an anechoic chamber with high-power heating or cooling condition and sound field free transform system are summarized. Moreover, during the construction of the anechoic chamber, the following technical problems have been solved successfully. (1) Under $15 \mathrm{~kW}$ heating or cooling energy load being released by the sound source in the anechoic chamber, the adjusting to the temperature and the humidity can be completed. (2) Through the 
moving of the mobile sound absorption plane, two anechoic chambers can be combined into one anechoic chamber, one semi-anechoic chamber, or one octant semi-anechoic chamber. Its sound field characteristics conform to the standard ISO 3745. (3) During the normal state, the background noise in the anechoic chamber is less than $23 \mathrm{dBA}$.

\section{$10: 25$}

2aNSa6. Hand-held analyzer systems for building acoustics and sound intensity/sound power. Peter Larsen (Bruel \& Kjaer, Skodsborgvej 307, DK-2850 Naerum, Denmark, plarsen@bk.dk)

Two new applications have been developed for a hand-held analyzer: Building acoustics and sound intensity/sound power. The building acoustics application is designed for measurements of airborne and impact sound insulation and reverberation time. The sound intensity application is used for sound power measurements, noise source location, noise mapping, and building acoustics. Both applications we used with complete systems consisting of all the necessary parts to simplify the measurement task and provide the user with all results immediately at the measurement site. There is no need for postprocessing. The measurements can be done according to a wide selection of international and national standards. The paper will describe the use of both applications in solving a problem in building acoustics. The building acoustic application will be used to detect a problem with poor sound insulation of a wall. Then, the intensity technique is used to determine the cause of the problem. Both applications use a built-in noise generator in the hand-held analyser with wireless transmission to an amplifier/loudspeaker. The paper will focus on the various design features that have been incorporated to ensure easy, secure, and flexible use.

TUESDAY MORNING, 23 JUNE 1998

CASCADE BALLROOM I, SECTION C (W), 8:00 TO 9:55 A.M.

Session 2aNSb

\title{
Noise: Community Noise Annoyance from Entertainment and Other Sources
}

\author{
Jim Griffiths, Chair \\ Symonds Travers Morgan Limited, Mead House, Canteloupe Road, East Grinstead, West Sussex RH19 3DG, England
}

Chair's Introduction-8:00

Invited Paper

8:05

\begin{abstract}
2aNSb1. A Hong Kong approach to control noise from outdoor entertainment activities. P. S. Ng, Sam W. H. Wong, Y. K. Kam, and K. S. Chan (Environ. Protection Dept., The Government of the Hong Kong Special Administrative Region of the People's Republic of China, 46th Fl., Revenue Tower, Wan Chai, Hong Kong)

With 6.5 million people living on some 1000 square kilometers of land, about $70 \%$ of which are hilly terrains, one could imagine how difficult it would be in planning compatible land use. High-rise residential blocks surrounding sports ground are not uncommon in Hong Kong. They should co-exist harmoniously if the latter is intended solely for recreational sports. Hong Kong, being a cosmopolitan city, hosts many kinds of outdoor entertainment activities of cultures and many are held frequently in these sports grounds owing to the absence of purpose-built outdoor venues for these activities. With increasing environmental expectations, complaints against noise from these activities are on the rise. Special considerations will have to be struck between efficient use of land and adequate noise protection of the nearby noise-sensitive receivers. This paper describes the various outdoor entertainment activities being held in Hong Kong, their noise generation characteristics, and the approaches taken to control the noise.
\end{abstract}

\section{Contributed Papers}

\section{$8: 25$}

2aNSb2. Annoyance and health effects of entertainment noise. C. G. Rice (Inst. of Sound and Vib. Res., The Univ., Southampton SO17 1BJ, UK)

There is no doubt that over the past 20 years awareness in the possible adverse annoyance and health effects which can result from entertainment and leisure activities has increased, and the topic is becoming more worthy of research effort in its own right. A review of the literature has established certain knowledge base gaps in the causal relationships between such noise and its adverse effects. Indeed, apart from noise-induced hearing loss there appears to be a lack of in-depth research from which unequivocal quantitative conclusions can be drawn. While damage to hearing from certain activities has been established, in general nonauditory health effects are not apparent although annoyance effects and provoked violence are becoming increasing causes of major concern. However, bearing in mind the relatively low integrated noise exposures which personally ac- crue from participation in and exposure to entertainment and leisure noise activities, care should be taken not to overemphasize the adverse effects to the prejudice of the other positive benefits created by participating in such activities.

\section{8:40}

2aNSb3. Proactive concert sound management: The essential ingredient for community acceptance of outdoor concert venues. Richard G. Cann (Grozier Tech. Systems, Inc., 157 Salisbury Rd., Brookline, MA 02146-2032) and William J. Cavanaugh (Cavanaugh Tocci Assoc., Inc., Sudbury, MA 01776)

Residents living adjacent to outdoor music venues have often objected to sound emissions. With increased sound power now available from loudspeakers, the situation is exacerbated, threatening the existence of music venues. Venues have demonstrated that by using a fixed installation sys- 
tem to manage and control concert sound emissions effectively, the friction with the community can be reduced substantially. An essential ingredient of the amelioration process is making automatic sound emission reports available to amphitheater sound engineers and management as each concert proceeds. Sharing these same reports with citizen activists immediately after each concert helps develop trust. Case histories will be presented.

\section{8:55}

2aNSb4. Entertainment park noise control devices. Alessandro Cocchi and Giovanni Semprini (DIENCA, V.le Risorgimento 2, 40136 Bologna, Italy, alessandro.cocchi@mail.ing.unibo.it)

A big entertainment park is operating with noisy attractions near the Garda lake, a pleasant land where people go not only for amusement but also to relax, especially during holiday time. Noise measurements have been made during different periods of daytime and a noise map has been developed taking into account the difference of level between the top of the noisiest attraction and the windows of the most exposed house. Starting from this computerized model, and knowing the emission spectrum of the most relevant sources, it has been possible to design some noise control device useful for reducing the noise exposure level restricted to the exposed houses alone: This was possible combining earth barriers and a hot windstream.

\section{9:10}

2aNSb5. Comparison of predicted and measured noise levels for refinery units. Frank H. Brittain (Bechtel Corp., 50 Beale St., San Francisco, CA 94105) and Mark M. Gmerek (The Boeing Co., P.O. Box 3707, Seattle, WA 98124)

Predicting noise levels from new refinery units is a vital part of environmental assessment and designing units to meet noise limits. The accuracy of those noise predictions is a very important concern. The simplest way to assess the accuracy of predictions is to compare predicted and measured noise levels. This is usually difficult, because measured levels are strongly affected by noise from adjacent units and by atmospheric effects on sound propagation. Further, actual noise levels of significant sources often deviate from expected levels used in the noise prediction model. Thus to meaningfully compare predicted and measured levels, the actual noise source levels for the major sources, atmospheric conditions, and noise levels from adjacent units must all be accounted for. Predicted and measured levels are compared for two large refinery units. Measurements were made at locations where noise from adjacent units has little effect and close enough so that atmospheric conditions have little impact on the measured levels. Measured operational noise levels of major sources were used to update the noise prediction model. Accuracy of the predictions is evaluated.
2aNSb6. Acoustic modeling and simulation of an urban substation. Eduardo Bauzer Medeiros and Gia Kroeff (Dept. de Eng. Mecånica da UFMG, Av. Antônio Carlos 6627, 31270-901, Belo Horizonte-MG, Brazil, flugzbau@oraculo.lcc.ufmg.br)

Demographic growth has often resulted in a disorganized occupation of large urban areas. Regions which were previously empty started to be populated, frequently resulting in an increasingly more common proximity between residential construction and industrial installations. As a consequence, very serious environmental problems have occurred, including those caused by acoustic emission from industrial installations. A common problem these days is the disturbance caused by electric substations closely positioned to residential buildings. The disregard for acoustic effects during the design stage can still be observed in some of the older substation designs, particularly in areas previously far from city centers. In some countries, such as Brazil, a systematic acoustic evaluation of substations only started in the 1980s, with the main bulk of the work still under development. This paper describes some of the joint work being carried out on noise control by the researchers of Universidade Federal de Minas Gerais and CEMIG (the state power administration). The initial definition of a series of acoustic elements, followed by the discussion of the governing equations, is considered. The computational implementation of the resulting model is presented, aimed at the definition of the most convenient noise control strategy. Finally, a few results obtained at a real substation are considered, including some experimental results obtained during actual operation.

\section{9:40}

2aNSb7. Environmental pollution and noise control of two power generator sets placed into a recreation area. Victor Rastelli, Nila Montbrun, and Alexis Buoza (Departamento de Mecánica, Universidad Simón Bolivar, Caracas 1080-A, Venezuela)

This paper deals with the noise control of two power generator sets of $545 \mathrm{~kW}$ each, located in a Venezuelan resort. The generator sets made a noise level of $73 \mathrm{~dB}(\mathrm{~A})$ at a distance of $47 \mathrm{~m}$ from the source, where are located the guest rooms. This level was far from the legal regulations for allowable environmental conditions. A noise enclosure with air silencers was proposed to reach a final level of $53 \mathrm{~dB}(\mathrm{~A})$ at the guest accommodations. Two aspects must be taken into account, the noise reduction and the ventilation specifications of the generator sets, in order to keep the operational temperature. The final noise level was measured at $54 \mathrm{~dB}(\mathrm{~A})$ and the pressure loss of the silencers was well placed into the ventilation specifications. 


\title{
Session 2aPAa
}

\section{Physical Acoustics: Ducts and Tubes}

\author{
James P. Chambers, Chair \\ National Center for Physical Acoustics, University of Mississippi, Coliseum Drive, University, Mississippi 38677 \\ Chair's Introduction-9:25
}

\section{Contributed Papers}

9:30

2aPAa1. The effect of mass transfer on sound propagation in cylindrical tubes using the low reduced frequency approximation. Craig J. Hickey and Richard Raspet (Dept. of Phys. and Natl. Ctr. for Physical Acoust., Univ. of Mississippi, University, MS 38677, chickey@olemiss.edu)

The theory for sound propagation in a gas/vapor mixture contained in a cylindrical tube is investigated. The tube is assumed to have a rigid wall covered by a thin film of water. This thin film of water acts as a source/ sink for the mass and the heat associated with the vapor. This problem has been previously solved in the high- and low-frequency limits using the Rayleigh eigenmode method [Y. Mao and J. M. Sabatier, J. Acoust. Soc. Am. 96, 3254(A) (1994)]. However, the interpretation of these results is limited by their complexity. This formulation of the problem parallels the low reduced frequency approximation work of Tijdeman [J. Sound Vib. 39, 1-33 (1975)]. In contrast to the earlier results an easily interpreted analytical solution for the propagation constant is obtained. The parameters governing the propagation of the sound waves are the shear wave number, Prandtl number, Schmitt number, and the reduced frequency. The limits of small and large shear wave number provide useful insight into the behavior of the gas/vapor mixture. Examples of air/water and helium/ water mixtures will be discussed. [Work supported by ONR.]

\section{9:45}

2aPAa2. Measurement of the radiation impedance of a pipe with a circular flange. Jean-Pierre Dalmont (Laboratoire d'Acoustique, Université du Maine, 72085 Le Mans Cedex 9, France) and Cornelis J. Nederveen (Acacialaan 20, The Netherlands)

The radiation of an open pipe with an infinite flange, as well as that of an unflanged pipe has been explicitly calculated by, respectively, Norris and Sheng [J. Sound. Vib. 135(1), 85-93 (1989)] and Levine and Schwinger [Phys. Rev. 73, 383-406 (1948)]. The radiation of real tubes, i.e., with a finite wall thickness or a finite circular flange, is not accurately known. The aim of the present measurements is to determine the radiation of tubes with various flanges and their frequency dependence. The radiation impedance has been deduced from the measurements of the input impedance of a short tube. As the differences between the different situations can be very small, the impedance sensor must be accurately calibrated. Results are reproducible and are in good agreement with a calculation using a boundary elements method (software, Rayon). It is observed that the curves obtained are not limited by the case of the infinite flange and the case of the unflanged pipe. This is probably caused by a resonance effect due to the diffraction at the edges of the flange.

\section{0:00}

2aPAa3. Boundary-integral-equation methods for accurate calculation of acoustic fields. James B. Mehl (Dept. of Phys., Univ. of Delaware, Newark, DE 19716-2570, jmehl@udel.edu)

Acoustic fields near solid boundaries can be approximated by solutions of the Helmholtz equation subject to Neumann boundary conditions on the boundaries. For closed, axisymmetric geometries, the boundary-value problem can be expressed as a one-dimensional integral equation. Bound- ary shapes can be represented exactly in this formalism. With current code, numerical solutions can be obtained for shapes formed by rotating plane figures made up of mixtures of straight and circular line segments. Numerical solutions to the integral equation were determined by using cubic-spline approximations to the velocity potential $\Psi$. This procedure enabled both $\Psi$ and the tangential acoustic velocity $\Psi^{\prime}$ to be determined with high precision. In calculations of the fields within ducts near the orifices, with modest $(\sim 200)$ numbers of boundary elements, approximate values of $\Psi^{\prime}$ were found which were smooth over five orders of magnitude. The technique was used to calculate fields near the duct ends and to evaluate inertial and resistive end effects, for both baffled and unbaffled duct ends. The effects of rounding sharp edges has been evaluated through the use of circular-arc boundary elements. [Work supported in part by the Office of Naval Research.]

\section{0:15}

2aPAa4. Numerical study of sound field radiated from a circular duct with an open end. Zhichi Zhu, Anqi Zhou, Dongtao Huang, and Qing Guo (Dept. of Eng. Mech., Tsinghua Univ., Beijing, 100084, PROC, zzc-dem@mail.tsinghua.edu.cn)

A dispersion-relation-preserving (DRP) scheme for computational acoustics has been developed in recent years. This scheme is of high accuracy and can preserve the original dispersion relations of governing equations so that a good global acoustic solution can be ensured. However, the scheme is usually not only very complex but also problem dependent. In this paper, a unified DRP scheme for both internal and boundary points is developed for solving the sound field radiated from a circular duct with an open end. This unified DRP scheme is further applied to obtain the sound fields numerically in three different cases, namely, without flow and flange, without flow but with flange, and with flow and flange. In addition, relevant reflection efficiencies are also predicted at various Helmholtz numbers. Satisfactory comparisons of the predicted reflection efficiencies with data from other studies are illustrated. It is also shown that the numerical method with the DRP scheme presented in the paper is successful.

\section{0:30}

2aPAa5. Experimental investigation on nonlinear standing waves by two tube structure. Ke Liu and Dah-You Maa (Inst. of Acoust., Academia Sinica, P.O. Box 2712, Beijing 100080, PROC)

Nonlinear standing waves have been studied in a structure consisting of two lengths of tubing with different diameters. The measurement system of the experiment is presented. A new high-power loudspeaker driver $(300 \mathrm{~W})$ is used as a sound source to excite the large tube of $45 \mathrm{~mm}$ diameter through a small hole near one end which is closed. Preliminary nonlinear theory is proposed, and a resonance diagram of the tube structure is given. The main properties of nonlinear standing waves were obtained. At the end of smaller tube, a sound-pressure level (SPL) of nearly $177 \mathrm{~dB}$ has been obtained with a low distortion. The phenomena of saturation and properties of the output at the end of the smaller tube as well as their relation with the input were investigated. Increasing the diameter of 
the structure directly with a modern high-power loudspeaker produces high intensity in a more extended space. Experiments are in progress. [Work supported by the National Natural Science Foundation of China.]

\section{0:45}

2aPAa6. Influence of airflow on sound propagation in a curved tunnel. Hiroyuki Imaizumi and Takehiro Isei (16-3 Onogawa, Tsukuba, Ibaraki, 305 Japan)

Measurements were made to examine influences of airflow on propagation attenuation of sound in a curved tunnel. The speed of airflow varied in width direction of the cross section of tunnel, and the values were from around 1 to $3 \mathrm{~m} / \mathrm{s}$. Sound attenuation upwind was less than that downwind at high frequencies up to the receivers that are in sight of the source. The tendency agrees with sound attenuation measured in a straight tunnel with airflow [H. Imaizumi et al., J. JSSE 31, 228-239 (1992)]. Difference of the attenuation gradually became small in the same frequency region at receivers that are out of sight from the source. Numerical calculations by means of a ray tracing method were applied to propagation attenuation of sound in a curved two-dimensional plane. Transmission losses of sound energy calculated only from reflection times of the sound rays on the inner wall were compared under each propagation condition. The transmission loss downwind was larger than upwind up to the receivers that are in sight, while the difference of both became small at the further receivers due to the rapid increase of the reflection times of rays. The calculation results qualitatively agree with the experimental measurements.

\section{1:00}

2aPAa7. Acoustic propagation in the presence of an arbitrary flow. C. J. Peyret (ONERA/MFE/DSNA, 29 Ave. de la division Leclerc, 92322 Chatillon, Cedex, France)

The study of acoustic propagation in a lined duct in the presence of an arbitrary flow remains open. Duct acoustic propagation in the presence of flow is generally computed assuming some particular properties of the flow and simplifying the duct geometry to find an appropriate wave equa- tion. For instance, the Lilley or Phillip equation can be deduced this way. However, a unique partial differential equation based on the acoustic displacement vector allows a nonhomogeneous flow in an arbitrary duct geometry to be taken into account. This equation was first established by Galbrun in 1931. After recalling the Galbrun equation, a method to solve it is presented. The method uses the Lagrangian properties in order to apply the finite elements method. Different boundary conditions may be introduced. The solution of the Galbrun equation is compared to the solution of the Lilley equation in the case of modal propagation in a lined duct in the presence of a shear flow. Both results are in good agreement, and validate the method used to solve the Galbrun equation.

\section{1:15}

2aPAa8. Propagation of vibration waves in a pipe. Mihail-Dan F. P. Simion (Dept. of Machines - Tools, Univ. Polytechnica Bucharest, 313 Splaiul Independentei, 77206, Bucharest), Gabriela-Cristina D. Simion, and Florian-Paul Simion (Univ. Polytechnica Bucharest, 313 Splaiul Independentei, 77206, Bucharest, simion@freddy.cib.pub.ro)

Our studies concerning propagation of vibrations inside a pipe presume a behavior analysis for a continuous medium, but also a dynamic study of elastic medium. A diagram for describing the behavior of a viscoelastic medium is represented with emphasizing properties of material. In our case energy is transmitted through propagation of longitudinal pressure waves. Every particle of medium is vibrating around its equilibrium position. The relation for deformations dependence on tensions are determined, taking into account a medium with negligible viscosity. Propagation velocity for longitudinal and transversal plane waves are computed, by aiding Navier equations. A ratio between longitudinal waves propagation velocity and transversal waves propagation velocity equal to 1.87 for $v=0.3$ is obtained.

TUESDAY MORNING, 23 JUNE 1998

ASPEN ROOM (S), 9:25 A.M. TO 12:00 NOON

\title{
Session 2aPAb
}

\section{Physical Acoustics: Topics in Thermoacoustics}

\author{
Anthony A. Atchley, Chair \\ Graduate Program in Acoustics, Pennsylvania State University, Applied Science Building, P.O. Box 30, \\ State College, Pennsylvania 16804
}

Chair's Introduction-9:25

\section{Contributed Papers}

\section{9:30}

2aPAb1. Simple model for temperature gradient formation in a short stack. Ralph T. Muehleisen (Dept. of Civil, Environ., and Architect. Eng., Univ. of Colorado, Boulder, CO 80309) and Anthony A. Atchley (Penn State Univ., P.O. Box 30, State College, PA 16804)

There has been much analytical, numerical, and experimental work investigating steady-state thermoacoustic phenomenon. However, much less work has been done on time-dependent problems such as the evolution of the thermoacoustic temperature gradient in a short stack of plates.
If the time scale of the acoustics is much shorter than that of the thermodynamics of the stack itself, the acoustics can be assumed to be in steady state while the temperature gradient in the stack is formed. With this assumption, the thermoacoustic heat flux between the stack plates will be of the form $Q=\left(C_{1}+C_{2} \partial T / \partial x\right) p^{2}$. This gives rise to a temperature gradient that rises exponentially in time with a time constant proportional to the thermal conductivity of the stack and the acoustic pressure squared. There is an additional exponential temperature rise from viscous heating. The model is shown to match measured temperature gradients very well. [This work was supported by the Office of Naval Research and the American Association for Engineering Education.] 
ations not encountered in full-scale devices. For example, the high me-

2aPAb2. Performance measurements of a thermoacoustic refrigerator driven at high amplitudes. Matthew E. Poese and Steven L. Garrett (Grad. Prog. in Acoust., P.O. Box 30, State College, PA 16804, matt@sabine.acs.psu.edu)

Since the power density in a thermoacoustic device is proportional to the square of the acoustic Mach number, there is strong motivation to design thermoacoustic refrigerators to operate at larger pressure amplitudes. Measurements will be reported of a modified version of the space thermoacoustic refrigerator (STAR) [Garrett et al., "Thermoacoustic refrigerator for space applications," J. Thermophysics Heat Transfer 7, 595-599 (1993)] driven at pressure amplitudes two to three times as large as reported in 1993. The results of these measurements will be compared to both a DeLTAE computer model of the low-amplitude (linear) performance and one that includes turbulent flow in the duct regions of the device. An overview of the instrumentation, including a measurement of exhaust heat with an absolute accuracy of $65 \mathrm{~mW}$, will also be presented. [Work supported by Office of Naval Research.]

\section{0:00}

2aPAb3. Extended performance measurements for SETAC. Anat Grant and Steven L. Garrett (Penn State Univ., P.O. Box 30, State College, PA 16804, anat@sabine.acs.psu.edu)

The Shipboard Electronics ThermoAcoustic Cooler (SETAC) was built at the Naval Postgraduate School in Monterey, CA. In a demonstration at sea aboard the U.S.S. DEYo in April 1995, SETAC was used to provide 419 watts of useful cooling power across a stack temperature difference $T_{\text {hot }}-T_{\text {cold }}=30{ }^{\circ} \mathrm{C}$ with an acoustic input power of 227 watts. New data spanning a wider range of temperature difference and heat load will be presented and compared to a DELTAE model. [Work supported by the Office of Naval Research.]

\section{0:15}

2aPAb4. Solar/heat-driven thermoacoustic engine. Reh-lin Chen and Steven L. Garrett (Grad. Prog. in Acoust. and Appl. Res. Lab., Penn State Univ., P.O. Box 30, State College, PA 16804)

With its abundance and cleanliness, solar energy has been harnessed to generate power using various techniques. A thermoacoustic engine is described which was built, instrumented, and tested to demonstrate use of solar power to generate acoustic power. Sunlight is collected using a 3-ftdiam acrylic Fresnel lens and focused on one end of a ceramic "stack." This engine has a total length of $40 \mathrm{~cm}$ and resonates at around $420 \mathrm{~Hz}$. To permit testing on the abundant cloudy days in Pennsylvania, an electrical heater was also built and installed to provide a reliable thermal energy source for indoor measurements. Experimental results are in reasonable agreement with a DeLtAE model. When running in solar mode, as a $\frac{1}{4}$ wavelength open end resonator, the measured acoustic SPL can reach 120 $\mathrm{dB}$ easily at $1 \mathrm{~m}$ away from the open end on a clear day. The onset of sound generation can be achieved even with a 1-ft-diam Fresnel lens. While an impressive demonstration in terms of sound output, despite modest efficiency, real solar energy conversion will be improved with inert gas mixtures at higher pressures. [Work supported by ONR, lens provided by G. W. Swift.]

\section{0:30}

2aPAb5. Thermoacoustic refrigeration demonstration. Ray Scott Wakeland and Steven L. Garrett (Grad. Prog. in Acoust., Penn State Univ., State College, PA 16801, garrett@ sabine.acs.psu.edu)

A portable device for demonstrating thermoacoustic cooling is developed using off-the-shelf components: the Morel MW-142 5-in. woofer and the Selenium HL14-25 horn. Acoustic peak pressure amplitudes of $19 \mathrm{kPa}$ (19\% of mean pressure) have been measured (without stack) using atmospheric air as the working fluid. Measurements of spectra, quality factors, stack temperature differences, and heat pumping capacity are compared to DeltaE models. Use of off-the-shelf components leads to design consider- chanical resistance of the driver, which was intended for audio reproduction, dictates against making the driver coresonant with the resonator, as is common when the driver is designed for operation at a specific frequency. [Work supported by NSF and ONR.]

\section{0:45-11:00 Break}

\section{1:00}

2aPAb6. Precision measurements of thermoacoustics in a single pore. Gabriela Petculescu and Larry A. Wilen (Dept. of Phys. and Astron., Ohio Univ., Athens, OH 45701, wilen@helios.phy.ohiou.edu)

Continuing experiments designed to look at thermoacoustic effects in a single pore will be described. A volume-modulation technique is employed to measure the complex compressibility of a gas in the pore directly. When a temperature gradient is imposed on the pore, the complex compressibility is sensitive to thermoacoustic effects which can be measured to high precision. These effects depend on both thermal and viscous properties of the gas. Measurements on different gases and mixtures of gases will be presented and compared to predictions of the theory. [Work supported by Ohio University Research and Sponsored Programs.]

\section{1:15}

2aPAb7. Time-average temperature distribution in a circular thermoacoustic pore. George Mozurkewich (Ford Motor Co., Maildrop 3028, P.O. Box 2053, Dearborn, MI 48121-2053, gmozurke@ ford.com)

The time-average temperature distribution in a thermoacoustic pore, such as one pore of a thermoacoustic stack, is calculated. The analysis applies to straight pores of arbitrary shape but is limited to the acoustic and short-stack approximations. A partial differential equation for temperature is found by applying conservation of energy to the standard expression for time-average energy flux. Previous solutions for parallel-plate pores [G. Mozurkewich, J. Acoust. Soc. Am. 103, 380-388 (1998)] are extended to pores of circular section. A heat-transfer coefficient is defined by $h_{1}=q(z) / \Delta T(z)$, where $q$ is the rate of heat transfer into the pore interior per unit area of pore wall, $\Delta T$ is the temperature difference between pore wall and pore centerline, and $z$ is the axial coordinate. This coefficient is independent of $z$ provided $z$ is not too close to an end of the pore. Results will be given for the shapes of the radial eigenfunctions and for the variations of eigenvalues and $h_{1}$ with pore size and Prandtl number.

\section{1:30}

2aPAb8. Linear resonant duct thermoacoustic refrigerator having two regenerator stacks. Yong Tae Kim, Sang Joon Suh (Acoust. and Vib. Group, Korea Res. Inst. of Standard Sci., P.O. Box 102, Yusong, Taejon 305-600, Korea), and Min Gon Kim (Dept. of Phys., Hankuk Univ. of Foreign Studies, Wangsan, Mohyun, Yongin, Kyungki 449-791, Korea)

The measurements of acoustically generated temperature difference between the two ends of a TAC (thermoacoustic couple) in a resonant tube are made using the similar method suggested by Atchley et al. [J. Acoust. Soc. Am. 88, 251-263 (1990)] with a ceramic TAC cylinder. The linear duct was made of PVC pipe with an inner diameter of $30 \mathrm{~mm}$ and $680 \mathrm{~mm}$ long. Measurements are made for a frequency range of $150-350 \mathrm{~Hz}$ in atmospheric air along the TAC length from 2.5 to $10 \mathrm{~cm}$ with a $1-\mathrm{cm}$ step. The unknown information of ceramic can be obtained from nonlinear fitting of experimental data with the equation reported by Atchley et al. From the result of this experiment and prediction by theory it is found that the direction of acoustic heat transport is altered at the point of a quarter of a wavelength. From the hint of the above experiment, a newly designed thermoacoustic refrigerator having two regererator stacks is tested. 
coustic stack. The third term on the left-hand side (lhs) describes a shift in

2aPAb9. Theory of nonlinear acoustic waves in a thermoacoustic prime-mover. Pierrick Lotton, Vitalyi Gusev, Hélène Bailliet, and Michel Bruneau (Lab. d'Acoustique, IAM-UMR 6613, Univ. du Maine, av. O. Messiaen, 72085 Le Mans Cedex, France, lotton@laum.univ-lemans.fr)

An equation describing stabilization of oscillations in a thermoacoustic prime-mover was derived: $\quad \partial V / \partial t-V(\partial V / \partial \tau)+(A-D)(\partial V / \partial \tau)$ $=\sqrt{2 / \pi} A \int_{-\infty}^{\tau}\left(\partial V / \partial \tau^{\prime}\right)\left[d \tau^{\prime} /\left(\tau-\tau^{\prime}\right)\right]+V+D\left(\partial^{3} V / \partial \tau^{3}\right)$. In this equation, particles velocity $V$, "slow" time $t$, and "fast" time $\tau$ are normalized; parameters $A$ and $D$ depend on temperature distribution inside thermoa- resonance frequency of a half-wavelength resonator caused by a stack installation. The third term on the right-hand side (rhs) describes sound velocity dispersion when the stack is acoustically thin. The first term (if $A>0$ ) and the second term on the rhs describe sound amplification due to quasiadiabatic interaction of acoustic and thermal waves. The first term on the rhs contributes also to dispersion. Particular analytical solutions were found and computer modeling was applied for the analysis. Conditions when a shock front formation caused by acoustic nonlinearity (the second term on the lhs) is the dominant mechanism for the saturation of wave amplitude were determined. [Work supported by DGA.]

TUESDAY MORNING, 23 JUNE 1998

GRAND BALLROOM II (W), 7:45 TO 10:45 A.M.

\title{
Session 2aPP
}

\section{Psychological and Physiological Acoustics: From Ear Canal to Cortex-Clear Cuts and Old Growth (Poster Session)}

\author{
Robert S. Schlauch, Chair \\ Department of Communication Disorders, Univerity of Minnesota, 115 Shevlin Hall, Minneapolis, Minnesota 55455
}

\section{Contributed Papers}

\begin{abstract}
All posters will be on display from 7:45 a.m. to 10:45 a.m. To allow contributors an opportunity to see other posters, contributors of odd-numbered papers will be at their posters from 7:45 a.m. to 9:15 a.m. and contributors of even-numbered papers will be at their posters from 9:15 a.m. to 10:45 a.m. To allow for extended viewing time, posters will remain on display until 12:00 noon on Wednesday, 24 June. A cash bar will be set up near these posters from 6:15 p.m. to 7:30 p.m. on Tuesday, 24 June.
\end{abstract}

2aPP1. Temporal versus spectral cues in AM detection. Martin E. Rickert and Neal F. Viemeister (Dept. of Psych., Univ. of Minnesota, 75 East River Rd., Minneapolis, MN 55455)

Several recent studies have shown that modulation thresholds for detecting sinusoidal AM are approximately constant out to much higher modulation frequencies for tonal carriers than for broadband noise carriers. It has been suggested that with tonal carriers there is a range of modulation frequencies for which both temporal (envelope) cues and spectral cues are used for detection. The present study attempts to assess the relative importance of these cues. Modulation thresholds for AM, quasifrequency modulation (QFM), and beats were measured for modulation frequencies of $8-512 \mathrm{~Hz}$ and carrier frequencies of 2 and $5 \mathrm{kHz}$. The results indicate that at intermediate modulation frequencies, AM thresholds are approximately constant while those for QFM and for beats decrease with increasing modulation frequency. This indicates that the detection of AM is not based solely on temporal cues. The implications of these findings for envelope detection and modulation filter-bank models will be discussed. [Work supported by NIDCD Grant No. DC00683.]

2aPP2. Estimates of temporal resolution at low and high spectral frequencies. David A. Eddins (Psychoacoust. Lab., Dept. of Speech and Hearing Sci., Indiana Univ., Bloomington, IN 47405, deddins@indiana.edu)

The auditory system must encode dynamic acoustic stimuli throughout the audible spectrum. This coding is limited by the temporal resolution of the ear. Unfortunately, the results of several investigations disagree on the way in which temporal resolution varies with stimulus frequency. While gap-detection thresholds measured with sinusoidal signals change little over the frequency range of 200-2000 Hz, gap and amplitude modulation detection with narrow-band noise is more variable. Eddins [J. Acoust. Soc. Am. 93, 470-479 (1993)] showed that modulation detection is not frequency dependent from 500 to $4400 \mathrm{~Hz}$, however, there are few measures of modulation detection above this frequency range. In the present experiment, thresholds for modulation depth were obtained as a function of modulation frequency. Narrow-band noise carriers had upper- cutoff frequencies ranging from 3200 to $12800 \mathrm{~Hz}$ and bandwidths from 400 to $3200 \mathrm{~Hz}$. Temporal modulation transfer functions (TMTF) demonstrated the typical low-pass characteristic. The form of the TMTF did not vary with noise bandwidth or frequency region from 3200 to $9600 \mathrm{~Hz}$. Sensitivity to modulation increased with increasing bandwidth. For lower modulation frequencies, thresholds were slightly reduced at $12800 \mathrm{~Hz}$. These results indicate that modulation detection varies little over a very broad range of frequencies.

2aPP3. Detecting irregularity of envelope modulation. Stanley Sheft (Parmly Hearing Inst., Loyola Univ. Chicago, 6525 N. Sheridan Rd., Chicago, IL 60626)

The ability to detect periodic irregularity in the temporal envelope of a modulated stimulus was measured with a 2IFC procedure. Irregular amplitude modulation (AM) was generated with a periodic phase modulation (PM) of the sinusoidal modulator of wideband noise. The modulation index of the PM was adaptively varied in terms of delta envelope rate to determine the threshold to just detect the PM of the sinusoidal amplitude modulation (SAM). Base SAM rate ranged from 4 to $256 \mathrm{~Hz}$. For each SAM rate, the PM rate ranged from 2 to half the SAM rate. The PM waveshape was either sinusoidal or had linear ramps with slope varied across conditions. At low SAM rates, PM waveshape had little effect with 
thresholds across conditions rising with PM rate. At higher SAM rates, an effect of waveshape was obtained; thresholds were highest at lower PM rates with a steeply sloping linear-ramped PM. For the other PM waveshapes at the higher SAM rates, thresholds were roughly constant across change in PM rate. Results suggest two rate regions in processing AM with the regions separated at roughly the corner frequency of the temporal modulation transfer function. [Work supported by NIH and AFOSR.]

2aPP4. Effects of carrier level on the processing of highly detectable envelope fluctuations. Magdalena Wojtczak and Neal F. Viemeister (Dept. of Psych., Univ. of Minnesota, 75 East River Rd., Minneapolis, MN $55455)$

A modified 2AFC matching procedure was used to measure the modulation depth that yielded similar perceived envelope fluctuations for different carrier levels. In one case, the standard was a $60-\mathrm{dB}$ SPL $1-\mathrm{kHz}$ tone that was amplitude-modulated at $16 \mathrm{~Hz}$, with a modulation depth that produced highly detectable envelope fluctuations. The comparison had the same carrier and modulation frequencies as the standard, and its level ranged from 15 to $85 \mathrm{~dB}$ SPL. The data indicate that the higher the carrier level, the less modulation was needed to produce the same perceived fluctuation depth. For broadband noise carriers, however, the data indicate that approximately the same modulation depth was needed to obtain subjectively similar envelope fluctuations for all carrier levels tested. These results are similar to those observed for detection of AM, and they suggest that the level effects seen for detection extend to suprathreshold AM processing, i.e., Weber's law for broadband noise and a "near-miss" for sinusoidal carriers. [Work supported by NIDCD Grant No. DC00683.]

2aPP5. Variations of the leaky-integrator model and validation with detection of sinusoidal and percentage duty-cycle modulated noise. John A. Nelson (Commun. Sci. and Disord., Jesse Jones Commun. Ctr., The Univ. of Texas, Austin, TX 78712-1089, john-a-nelson@uiowa.edu) and Ruth A. Bentler (The Univ. of Iowa, Iowa City, IA 52242)

The leaky-integrator model is commonly used to describe auditory temporal processing. In the current study, the low-pass filter and decision device of the model were investigated. Maximum-to-minimum envelope magnitude, rms envelope power, envelope fourth moment, and envelope crest factor were implemented as potential decision devices [E. A. Strickland and N. F. Viemeister, J. Acoust. Soc. Am. 99, 3638-3646 (1996)]. Model predictions demonstrated interaction between the decision device and low-pass filter cutoff frequency. To validate model variations, detection thresholds of sinusoidal and percentage duty-cycle modulated broadband noise were measured at octave frequencies $2-512 \mathrm{~Hz}$ for listeners with normal hearing. To ensure subjects responded exclusively to temporal cues, the modulated noise was compensated for overall average power. Further, an intensity roving technique was used between observation intervals. The data show low-pass transfer function characteristics across all modulation functions. A two-line regression fit of the data revealed that the cutoff frequency, slope, and overall gain varied as a function of the duty-cycle modulation function. The nonlinear growth of the various decision devices as a function of the low-pass filter is used to explain individual variability among subjects. [Work supported, in part, by the American Academy of Audiology Student Investigator Award.]

2aPP6. Comodulation masking release with tone pips added out of phase to the valleys of a sinusoidally amplitude modulated pure tone. Deborah A. Fantini, Roel Delahaye, and Ray Meddis (Dept. of Psych., Essex Univ., Wivenhoe Park, Colchester CO4 3SQ, England, deb@essex.ac.uk)

Two studies of comodulation masking release (CMR) investigated the influence of temporal position of brief signals added to a modulated onfrequency band (OFB) [Grose and Hall III, J. Acoust. Soc. Am. 85, 12761284 (1989); Moore et al., ibid. 87, 1683-1694 (1990)]. The former re- ports mixed results as to whether flanking bands (FBs) modulated to the same depth as the OFB elicit more CMR than 100\%-modulated FBs. The latter reports that when antiphase signals are added to valleys of $50 \%$ modulated OFBs, thresholds with correlated FBs are poorer than with quadrature-phase FBs. In the present paper, signals were three antiphase tone pips added to valleys of a $50 \%$-modulated $700-\mathrm{Hz}$ pure tone. Thresholds were obtained with the OFB and $6 \mathrm{FBs}$, modulated at $10 \mathrm{~Hz}$. FBs were comodulated or in quadrature phase with the OFB, at either $50 \%$ or $100 \%$ depth. With 50\% FBs, subjects performed better with comodulated than with quadrature-phase FBs. With $100 \%$ FBs, performance in the comodulated condition improved, and in the quadrature-phase condition was impaired, relative to $50 \%$ FBs. These results indicate that CMR can occur when antiphase signals are added to valleys of $50 \%$-modulated OFBs.

2aPP7. Comodulation masking release: The effect of the characteristics of noise bands presented before and after a signal. Lee Mendoza (Dept. of Commun. Sci. and Disord., Louisiana State Univ., 163 M\&DA, Baton Rouge, LA 70803, 1mendoz@unix1.sncc.lsu.edu), Joseph W. Hall III and John H. Grose (Univ. of North Carolina, Chapel Hill, NC 27599)

CMR was measured as a function of masker duration in three conditions. In the first condition, a 400-ms pure-tone signal was presented in the temporal center of comodulated masking bands ranging in duration from 400 to $1200 \mathrm{~ms}$. No effect of masker duration was observed: CMR was equivalent to that found in continuous maskers. In the second condition, continuous independent bands identical in spectra to the comodulated bands were gated off before, and on following, the comodulated bands. The presence of the independent bands reduced CMR considerably. Increasing the duration of the comodulated bands increased CMR somewhat; however, CMR was still substantially smaller than that observed without the independent bands. In the third condition, band limited noise spanning the same range as the comodulated bands was substituted for the independent bands. The bandlimited noise had little effect on CMR. In a second experiment, independent bands were gated on for various durations preceding spectrally identical $400-\mathrm{ms}$ comodulated bands masking a 400-ms signal. As the duration of the independent bands increased, CMR decreased. Based on these results, it appears that CMR is not entirely the result of processing only information concurrent with the signal. [Work supported by NIH-NIDCD and Louisiana Board of Regents.]

2aPP8. Perception of roughness in harmonic multitones. Andrzej B. Dobrucki and Maurycy J. Kin (Inst. of Telecommunication and Acoust., Wroclaw Univ. of Technol., Wyb. Wyspianskiego 27, 50-370 Wroclaw, Poland)

This paper presents results and problems of perception of mixed modulation (MM) in harmonic multitone for higher modulating frequencies $(70$ and $200 \mathrm{~Hz}$ ), and a 5-harmonics complex. Results indicated that perception of frequency modulation at the presence of the threshold values of amplitude modulation is different than the perception of amplitude modulation at the presence of the threshold values of frequency modulation. It means that two mechanisms of perception for these kinds of modulation exist. It turns out that for perception of this kind of modulation, the hearing system uses two sidebands produced by modulation process, but the high sideband seems to be more important than the low one. Another phenomenon is the perceptual grouping mechanism: for some of the modulated partials MM can be perceived as an additional pitch occurring in multitones, which usually is out of tune for fundamental frequency of the complex. The summation of the partials in one critical band takes place, which suggests that a specific pitch for that partial could be changed 
and this is a simple way to detect these simultaneous fast changes in the amplitude and frequency of the modulated components from the musical point of view.

2aPP9. A model of auditory image flow. I. Architecture. Neil P. McAngus Todd (Dept. of Psych., Univ. of Manchester, Manchester M13 9PL, UK, todd@hera.psy.man.ac.uk)

A model is presented here based on the theory that the auditory cortex computes the spatio-temporal power spectrum of the auditory image flow [D. Heeger, "Model for the extraction of image flow," J. Opt. Soc. Am. 4, 1450-1470 (1987)]. A computational implementation of the model has the following components: (1) a gammatone filter-bank spaced on the ERB rate scale; (2) an orthogonal, ERB spaced modulation filter-bank which generates a 2-D modulation map which is then low-pass filtered; and (3) a population of causal 3-D spatio-temporal Gabor filters which compute the spatio-temporal power spectrum of the image sequence. The cortical filters are organized into families such that each member of the family occupies the same spatial-frequency band but has a different spatio-temporal orientation. The spatial frequencies range from 0.05 cycles erb $^{-1}$ to 0.4 cycles $\mathrm{erb}^{-1}$. The temporal frequencies range from \pm 0.5 to $\pm 64 \mathrm{~Hz}$. The spatiotemporal power spectrum may be visualized by replacing each image pixel with the output of all filters centered at the pixel arranged in frequency and orientation bands, somewhat like orientation columns in the visual cortex. This model may be applied to modulation perception and auditory grouping.

2aPP10. A model of auditory image flow. II. Detection of amplitude and frequency modulation. Neil P. McAngus Todd (Dept. of Psych., Univ. of Manchester, Manchester M13 9PL, UK, todd@hera.psy.man.ac.uk)

In order to evaluate the cortical model proposed in Todd [these proceedings] it was applied to the phenomenon of modulation detection. It was assumed that a discrimination task involves the correlation of the spatio-temporal power spectra of two image sequences, such as may occur in a typical 2A-2IFC procedure. The correlation was computed by $k(1-r)$, where $r$ is the product moment correlation, for four different modulation frequencies, 4, 16, 64, and $256 \mathrm{~Hz}$ at four modulation depths for both AM and FM of a 1000-Hz carrier of 1000-ms duration against the image power spectrum of an unmodulated $1000-\mathrm{Hz} 1000-\mathrm{ms}$ tone. The obtained measures were then linearly regressed (with no intercept) as a function of the square of the modulation index. In all cases $k(1-r)$ gave a good fit $\left(R^{2}>0.95\right)$ for both AM and FM. For each of the modulation frequencies the slope of the regression could be used to estimate the model thresholds. The model shows the correct behavior for AM and FM detection. This mechanism, it is suggested, may provide an account of modulation detection interference since low spatial-frequency filters span several ERBs.

2aPP11. Role of pinna cavities in median plane localization. Kazuhiro Iida (AVC Res. Lab., Matsushita Comm. Ind., 600 Saedo, Tsuzuki, Yokohama, 224 Japan, kiida@adl.mci.mei.co.jp), Motoki Yairi, and Masayuki Morimoto (Kobe Univ., Nada, Kobe, 657 Japan)

This paper investigates the role of pinna cavities in median plane localization. First, sound image localization tests were carried out under the conditions of progressively occluding three pinna cavities: scapha, fossa, and concha. Second, HRTFs were measured under the same conditions as those of the localization tests. Finally, the results of the localization tests and those of HRTFs measurements were compared. The results of the localization tests and the HRTFs measurements show: (1) Concha plays a great role in median plane localization regardless of the source elevation. (2) The amplitude spectrum of HRTFs shows a significant change in cases of concha occlusion. The comparisons of these results suggest that (3) the spectral cue in median plane localization is mainly formed by concha. (4) However, the degree of localization error which was caused by the occlusion of the pinna cavities depends on the subjects.

2aPP12. The influence of different speech segments on spatial localization. Brian L. Karlsen (Ctr. for Personkommunikation, Aalborg Univ., Frederik Bajersvej 7, DK-9220 Aalborg, Denmark, blyk@get2net.dk)

Numerous researchers have investigated to what degree humans are able to localize different sounds in the horizontal plane. Much of this work has focused on human response to simple sounds, like pure tones, white noise, and click trains, the underlying assumption being that responses to more complex stimuli could be inferred from a combination of responses to the simple stimuli. It was decided to investigate this for speech segments. Specifically, it was hypothesized that subjects would display a difference in localization ability for CVs containing some consonant manner groups rather than others. A number of Danish CVs were used as target stimuli in a horizontal localization task. The CVs were simultaneously masked by a diffuse white noise field. All subjects were normally hearing. The subjects were asked to determine from which clock position they heard a speaker, and to respond as fast as possible by pressing the corresponding button on a clock face. Final results are expected to confirm the hypothesis in full and indicate that there is indeed a difference in localization ability between the different consonant manner groups.

2aPP13. Underwater sound localization: Role of interaural time and intensity differences. Pierre Bovet (Lab. de Psych. Cognit., Univ. de Genéve, 9 route Drize, 1227, Carouge, Switzerland), Carolyn Drake (CNRS URA 316, Univ. Rene Descartes, 75006, Paris, France), François Bernaschina (Univ. de Genéve, Carouge, Switzerland), and Sohpie Savel (CNRS URA 316, Paris, France)

In theory, underwater sound localization is very limited compared with that in air. Most cues are greatly reduced underwater: Interaural time differences are divided by four (due to faster sound propogation in water) and intensity differences are greatly reduced because signal conduction to the inner ear mainly occurs through skull bone condition, making the head transparent. However, preliminary studies indicate some localization abilities in scuba divers. An azimuth identification task was conducted in Lake Leman. At a depth of $10 \mathrm{~m}, 14$ divers indicated which of eight transducers located every 45 was generating a sound $(400 \mathrm{~Hz}, 6 \mathrm{kHz}$, or white noise). Results indicate: (a) correct estimates were highest for lateral positions (left and right) and poor for median positions (front and back), (b) performance was high for $400 \mathrm{~Hz}$ and white noise but low for 6-kHz signals, and (c) head movements did not appear to facilitate localization. Thus the best localization abilities were observed in positions with highest interaural time differences, suggesting that, contrary to theoretical predictions, temporal binaural cues must be at least partially functional. However, the fact that localization was poor for high-frequency signals suggests that interaural intensity differences do not play a significant role.

2aPP14. A new model for binaural signal detection. Jeroen Breebaart, Steven van de Par, and Armin Kohlrausch (IPO-Ctr. for Res. on UserSystem Interaction, P.O. Box 513, NL-5600 MB Eindhoven, The Netherlands)

Most present-day binaural models make use of coincidence detectors following an internal delay, so-called crosscorrelation models. We present a new modeling approach based on a subtractive mechanism as found in excitatory-inhibitory type neurons in the auditory pathway. This approach transforms, for each auditory filter, time-domain waveforms into a timevarying two-dimensional activity pattern, where each unit within that pattern has a characteristic interaural time delay and intensity difference. Limits of resolution are modeled by adding the same amount of internal noise to each unit. From this activity pattern, several features of the stimu- 
lus can be extracted, such as the apparent lateralization and the presence of a test signal within a masker. For modeling binaural detection it is assumed that a test signal must induce a significant change within the internal representation in order to be detectable. Depending on the condition, detection is limited by internal noise $(\operatorname{NoS} \pi)$ or by external fluctuations $(\mathrm{N} \rho \mathrm{S} \pi, \rho \leqslant 0.95)$. This model can be used as an artificial observer in the same way as described by Dau et al. [J. Acoust. Soc. Am. 99, 3615-3622 (1996)] for monaural signals.

2aPP15. Further evidence for the influence of peripheral compression on binaural detection. Steven van de Par and Armin Kohlrausch (IPO-Ctr. for Res. on User-System Interaction, P.O. Box 513, NL-5600 MB Eindhoven, The Netherlands)

Binaural detection at high frequencies depends on the envelope statistics of the masker. Van de Par and Kohlrausch [J. Acoust. Soc. Am. 101, 3104 (1997)] proposed that this results from the compressive response of the basilar membrane which can be shown to reduce interaural intensity differences (IIDs) strongest in stimuli with flat masker envelopes. To further test the effect of compression, the same experiment was repeated at $500 \mathrm{~Hz}$. Results show that binaural detection at low frequencies is much less dependent on masker envelope statistics as compared to high frequencies. This was expected because at low frequencies, besides IIDs, interaural time delays can be used for signal detection, which are not affected by compression. In addition, psychometric functions for the discrimination between 4-kHz NoSo and $\operatorname{NoS} \pi$ stimuli were measured as a function of the $\mathrm{S} / \mathrm{N}$ ratio for stimuli of 20 and $65 \mathrm{~dB}$ SPL using a 250-Hz-wide masker. The psychometric functions were rather symmetric around a $0 \mathrm{~dB}$ S/N ratio at low SPLs, but asymmetric at $65 \mathrm{~dB}$ SPL favoring discrimination at a negative $\mathrm{S} / \mathrm{N}$ ratio. Such an asymmetry at high stimulus levels is predicted, if the detection cue is a change in the interaural correlation of the compressed envelopes.

2aPP16. A model of auditory localization in noise using neural networks. Scott K. Isabelle (Dept. of Psych., Wright State Univ., Dayton, OH 45435), James A. Janko (Ball Aerosp. and Technologies Corp., Fairborn, OH 45324), and Robert H. Gilkey (AL/CFBA, Wright-Patterson AFB, Dayton, OH 45433-7901)

Janko et al. [Binaural and Spatial Hearing in Real and Virtual Environments, edited by Gilkey and Anderson (Erlbaum, Hillsdale, NJ, 1996), pp. 557-570] used neural networks to describe auditory localization and found that data in quiet were insufficient to discriminate among models. To further constrain the models, localization in the presence of a spatially fixed masker is considered here. The wideband targets and maskers were filtered by head-related transfer functions, then by a gammatone filter bank. A model for binaural interaction similar to that of Lindemann [J. Acoust. Soc. Am. 80, 1608-1622 (1986)] was used to process the filterbank outputs. The inhibited cross-correlation output of the binaural processor was sampled and used as input to a neural network (e.g., 24 correlation lags at each of 13 frequency channels for 312 input nodes). The network was trained using back propagation to estimate the target location in quiet, then tested on targets in noise at several values of signal-to-noise ratio (SNR). The network was also trained across several values of SNR. Results are compared to localization in noise by humans [Good and Gilkey, J. Acoust. Soc. Am. 99, 1108-1117 (1996)]. [Work supported by AFOSR F49620-95-1-0106 and the Ohio Board of Regents.]

2aPP17. On the difference between absolute and relative auditory distance perception. Hae-Young Kim, Yôiti Suzuki, Shouichi Takane, Kenji Ozawa, and Toshio Sone (Res. Inst. Elec. Comm., Tohoku Univ., Sendai-shi, 980-8577 Japan)

Absolute distance perception in the absence of information about intensity and reflections from boundaries is quite difficult [B. G. Haustein, Hochfrequenztech. u. Elektroakustik 78, 46-57 (1969)] but is possible to achieve if a sound source exists within the distance of around 1 to $2 \mathrm{~m}$ from a listener. In the presence of multiple sound sources, however, some cues can be derived by comparing information from different sources. From this point of view, it is important to study relative distance perception between sounds from multiple sources to investigate general distance perception in auditory system. Thus using the same experimental setup, two kinds of listening experiments about the absolute and relative distance perception were conducted. In the experiment for relative distance perception, a subject was asked to report the perceived distances of two sound sources presented successively with an interval of $2 \mathrm{~s}$ by using a rating scale with seven categories. The results of absolute distance perception showed that the perceived distance of a sound image significantly increases with the increase of actual distance of the sound source up to around $1.2 \mathrm{~m}$. On the other hand, the results of the relative auditory distance perception showed that the perceived distance significantly increases up to 2 to $3 \mathrm{~m}$.

2aPP18. Auditory motion aftereffects with a two-component adapter. Hisashi Uematsu and Makio Kashino (NTT Basic Res. Labs., 3-1, Morinosato-Wakamiya, Atsugi, Kanagawa, 243-01 Japan)

Auditory motion aftereffects have been used to investigate mechanisms used to detect changes in interaural time differences (ITDs). In this paper, auditory motion aftereffects with two-tone adapters were used to examine how the ITD-change information from each frequency band is integrated. In the experiment, a complex tone with 250 - and $500-\mathrm{Hz}$ components was used as an adapter. The magnitude of the auditory motion aftereffect for a physically stationary $500-\mathrm{Hz}$ test tone was measured using various combinations of direction and adapter component. Apparent sound movement was produced by varying only ITDs. The results showed no difference in the magnitude of the aftereffect between the $500-\mathrm{Hz}$ tone adapter and the complex adapter when the two components moved toward the same direction. On the other hand, the aftereffects disappeared when the two components of the adapter moved in opposite directions. This suggests that the integration of ITD-change information from each frequency band is not a simple linear summation. Mechanisms of acrossfrequency integration will be discussed.

2aPP19. Echolocation by moving and stationary listeners. Michael S. Gordon, Lawrence D. Rosenblum, and Luis Jarquin (Dept. of Psych., Univ. of California, Riverside, Riverside, CA 92521, rosenblu@ citrus.ucr.edu)

It has long been known that human listeners can echolocate a sound reflecting surface as they walk towards it [e.g., Supa et al., Am. J. Psychol. 57, 133-183 (1944)]. There is also evidence that stationary listeners can determine the location, shape, and material of nearby surfaces from reflected sound [Rice, Science 155, 656-664 (1967)]. The current research tested whether there is an advantage of listener movement for echolocating as has been found for localization of emitted sounds [Ashmead et al., J. Exp. Psychol.: Hum. Percept. Perform. 21, 239-256 (1995)]. Two groups of blindfolded subjects were asked to echolocate a $3 \times 6 \mathrm{ft}$ wall while either moving or stationary. After echolocating, the wall was removed, and subjects were asked to walk to where the wall had been. Subjects in the moving and stationary groups echolocated for the same amount of time and for each of four distances. Results showed that moving group subjects were more accurate than stationary group subjects for some distances. These results might be a function of useful time-to-arrival acoustic information available to moving listeners. 
2aPP20. Reflectance measurements of the contralateral acoustic reflex input-output function. M. Patrick Feeney (Ohio Univ., School of Hearing and Speech Sci., Lindley Hall 218, Athens, OH 45701) and Douglas H. Keefe (Boys Town Natl. Res. Hospital, Omaha, NE 68131)

Available data suggest that the acoustic reflex reduces the transmission of sound through the middle ear in humans at frequencies below $1000 \mathrm{~Hz}$. Wideband $(0.2$ to $10 \mathrm{kHz})$ reflectance, complex admittance, and absorbed power were used to measure the input-output function of the contralateral acoustic reflex in four adults. Broadband noise was used as a reflex activator. Measurements were taken at reflex threshold and in 5-dB increments above up to $112 \mathrm{~dB}$ SPL in one subject. The power transmission loss grew approximately $2.5 \mathrm{~dB}$ for every 5 - $\mathrm{dB}$ increase in the activator above reflex threshold. Admittance measurements generally mirrored these changes below $1000 \mathrm{~Hz}$ with decreases in equivalent volume, conductance, and complex admittance. There was an increase in admittance with the acoustic reflex at frequencies from 1000 to $4000 \mathrm{~Hz}$ in agreement with Rabinowitz [Ph.D. thesis, MIT, 1977]. An unexpected decrease in energy reflectance was observed for one subject in a notch centered at $5600 \mathrm{~Hz}$, near the zero crossing for equivalent volume, which suggests that the activation of the acoustic reflex increased the power delivered to the middle ear at that frequency. [Work supported by Ohio University Research Committee Grant No. 97-14, Ohio University CHHS SA Award, and NIH Grant No. DC00520.]

2aPP21. Vibration measurement of the tympanic membrane using the time-averaged speckle pattern interferometry. Hiroshi Wada, Masataka Takeuchi, Koji Hozawa (Tohoku Univ., Sendai, Japan), Takashi Gemma (Nikon Corp., Tokyo, Japan), and Makoto Nara (Nikon Technologies, Inc., Tokyo, Japan)

In this study, using the time-averaged electronic speckle pattern interferometry, the sinusoidal phase modulation technique by a direct modulation of laser injection current, and the digital image processing technique, an attempt was made to develop a system for measuring a small vibration in nanometer order. The vibratory responses of the guinea pig's tympanic membrane were measured. At the frequency of $1500 \mathrm{~Hz}$, the whole part of the tympanic membrane vibrated almost in phase. The maximum amplitude was about $30 \mathrm{~nm}$. At the frequency of $2500 \mathrm{~Hz}$, the tympanic membrane had two local maxima in the posterior and inferior portions. At the frequency of $3000 \mathrm{~Hz}$, the four peaks around the manubrium were seen. The number of the peaks increased with an increase in frequency and the vibration mode became more complicated. The results of this study indicate that the vibration amplitude is detectable in nanometer order by this system even when the stimulus level is small, e.g., $60 \mathrm{~dB}$ SPL. Consequently, the system developed by our group has a potential for clinical applications. [Work supported by the Ministry of Education of Japan.]

2aPP22. Filter shapes for brief signals as a function of preceding stimulation. Elizabeth A. Strickland (Dept. of Audiol. and Speech Sci., Purdue Univ., West Lafayette, IN 47907)

The threshold for a brief probe tone in a broadband noise was measured as a function of probe frequency $(1$ or $4 \mathrm{kHz})$ and masker level (0-40 dB spectrum level). The probe was always presented $2 \mathrm{~ms}$ after the onset of the masker, and the masker was preceded either by silence, or by a precursor with the same spectrum and level as the masker. For each subject there were some conditions in which probe threshold was higher with no precursor than with a precursor (overshoot), and some conditions in which probe threshold did not differ with the presence of a precursor. Filter shapes for the same brief probes were then measured using the notched noise method. The probe was always presented with a 2-ms delay from the onset of the notched-noise masker. The masker was preceded by silence or by a precursor. The probe level was set $10 \mathrm{~dB}$ above the threshold measured for a given condition in the overshoot experiment. A comparison will be made of filter shape and the presence or absence of overshoot. [Work supported by NINCDS.]
2aPP23. Variable-duration notched-noise experiments in a broadband-noise context. Jim J. Hant, Brian P. Strope, and Abeer A. Alwan (Speech Processing and Auditory Percept. Lab., Dept. of Elec. Eng., UCLA, 66-147E Eng. IV, 405 Hilgard Ave., Los Angeles, CA 90095)

To model the confusion of short-duration speech signals in noisy environments, it is important to characterize how humans resolve the spectral components of short-duration signals in background noise. Toward that end, a variable-duration notched-noise experiment was conducted in a noise context. Broadband noise preceded and followed a tone and notched noise of similar duration. Thresholds were measured at four durations (10, $30,100$, and $300 \mathrm{~ms})$, two center frequencies $(0.6,2.0 \mathrm{kHz})$, and five relative notch-widths $(0.0,0.1,0.2,0.4,0.8)$. At $0.6 \mathrm{kHz}, 10$-ms thresholds decrease $6 \mathrm{~dB}$ across notch-widths while 300-ms thresholds decrease over $35 \mathrm{~dB}$. These durational trends are similar but less pronounced at $2 \mathrm{kHz}$. In a second experiment, the short-duration notched noise was replaced with a flat noise, which provided an equivalent amount of simultaneous masking to that of the first experiment, and thresholds dropped by as much as $20 \mathrm{~dB}$. A simple filtering model with either a linear or nonlinear combination of simultaneous and nonsimultaneous masking is unable to predict these results. Instead, it appears that the elevated thresholds at short durations are dependent on the spectral shape of the simultaneous masker, implying reduced frequency selectivity. [Work supported by NIH-NIDCD and The Whitaker Foundation.]

2aPP24. Comparison of psychophysical and single-unit thresholds in response to electrical stimulation of the cochlea in guinea pig. Bryan E. Pfingst, Lisa M. Montney, and Sanford C. Bledsoe (Univ. of Michigan, Kresge Hearing Res. Inst., 1301 East Ann St., Ann Arbor, MI 48109-0506, bpfingst@umich.edu)

Recent studies have shown that the spatial pattern of currents used on individual channels of cochlear implants can have a large effect on speech recognition and other measures of electrical-stimulus perception. This study is one of a series that seeks to understand the neural patterns of response underlying these effects. Guinea pigs were deafened in one ear by local perfusion of an antibiotic and implanted with an electrode array in the scala tympani. Psychophysical strength-duration functions (detection threshold versus electrical pulse duration) were obtained in guinea pigs trained using positive reinforcement, operant conditioning techniques. Single-unit data were obtained from inferior colliculus in the same animals, or in guinea pigs deafened and implanted in a similar manner. In these preparations, thresholds for neural responses were near the psychophysical thresholds at all phase durations, and the thresholds for the most sensitive neurons were several $\mathrm{dB}$ below the psychophysical detection thresholds. Thus, the paradoxical discrepancies between neural responsiveness and behavioral stimulus detection observed in previous comparisons made across animal models do not occur in this preparation. This seems to be a useful preparation for determining the spatiotemporal patterns of neural activity that underlie electrical-stimulus perception. [Work supported by NIH-NIDCD Grant No. DC00274.]

2aPP25. Physiological correlates of the "mean-term" auditory adaptation. N. G. Bibikov (N. N. Andreyev Acoust. Inst., Moscow 117036, Russia)

In psychophysics, adaptation is defined usually as a decline of loudness of steady stimulus as a function of time. It occurs over periods ranging from seconds to minutes. Recent psychophysical data show that during a "mean-term" adaptation period (the first 30 seconds of the sound) the detection of amplitude changes considerably improves. Physiological correlates of "mean-term" adaptation in single units located in different regions (cochlear nucleus, superior olive, torus semicircularis) of the auditory system of the immobilized frog Rana temporaria are explored. Driven responses to long-duration pure tones and AM tones decreased over time periods ranging from milliseconds to minutes. The adaptation 
increased at the upper levels of the auditory system. The response to pure tone stimuli declined more rapidly than the response to AM tones. For AM stimuli the adaptation increased as the modulation depth decreased. The considerable enhancement of the phase locking to the sound envelope could be observed during the first $10-15 \mathrm{~s}$ of the AM tones with low modulation indexes. The effect of phase-locking enhancement during the mean-term adaptation matched some psychoacoustical findings. [Work supported by the RFBR.]

2aPP26. Mechanisms underlying recovery from forward masking in electrical stimulation. Monita Chatterjee (Dept. of Auditory Implants and Percept. Res., House Ear Inst., 2100 W. Third St., Los Angeles, CA 90057)

In normal hearing, adaptation is hypothesized to be closely related to recovery from masking. In electrical stimulation, adaptation at the inner hair cell synapse is absent. However, refractory mechanisms can result in an adaptationlike decrement at high stimulation rates. Shannon [ J. Acoust. Soc. Am. 88, 741-744 (1990)] found that normalized psychophysical forward masking functions were similiar in normal and electric stimulation, and suggested that the mechanisms underlying forward masking are primarily retro-cochlear. Recovery from forward masking was studied in five Nucleus-22 cochlear implant users. Results were found to be strongly subject-dependent. In general, a rapid decay in the first $15 \mathrm{~ms}$ after masker offset, and a slow decay persisting up to $200 \mathrm{~ms}$ after masker offset, were observed. Under some conditions, plateaus and nonmonotonicities were observed in the region of transition between the two processes. Recovery was independent of masker level. Masker duration, pulse rate, and maskerprobe spatial separation strongly influenced the relative weight of the rapid component. The amount of masking near masker offset did not determine the time course of recovery. It is hypothesized that the rapid mechanism is due to auditory nerve refractoriness, while the slow mechanism is likely to be more central in origin. [Work supported by NIDCD.]

2aPP27. Neurophysiological and psychophysical measures of duration discrimination in normal-hearing adults and adults with cochlear implants. Patricia G. Trautwein, Curtis W. Ponton, Betty Kwong, and Michael D. Waring (House Ear Inst., 2100 West Third St., Los Angeles, CA 90057)

The ability to discriminate duration contrasts (a first approximation of voice onset time differences) was evaluated in adult cochlear implant users and normal-hearing adults using a neurophysiological response (the mismatch negativity-MMN). The MMN is an evoked response generated by a deviant stimulus when embedded in a series of standard homogeneous stimuli. Magnitude of the MMN correlates with psychophysical discrimination thresholds indicating that the MMN provides a measure of perceived acoustic change [Kraus et al. (1996)]. The MMN thus provides a neurophysiological correlate of acoustic discrimination. The present study compared an objective statistical measure of MMN magnitude to psychophysical measures in response to duration differences of brief auditory stimuli. A ten-click train was paired with shorter trains to create an oddball sequence. The selected duration differences were similar to voice onset time differences that distinguish voiced from voiceless speech phonemes. The MMN magnitude was measured at four scalp-electrode locations. The relationship between neurophysiological and psychophysical thresholds was determined and comparisons were made between the normal-hearing adults and adults with cochlear implants. While some similarities were found, the results suggest that the sensitivity to duration differences differs between normal-hearing and cochlear implant subjects.
2aPP28. Spectro-temporal influence on auditory cortical evoked potential thresholds. Ann Clock Eddins (Auditory Physiol. Lab., Dept. of Speech \& Hearing Sci., Indiana Univ., Bloomington, IN 47405, aeddins@indiana.edu)

Previous studies from our laboratory have shown that auditory cortical evoked potential thresholds (N1-P2) decrease with increasing stimulus duration. Similar to behavioral studies of auditory temporal integration, N1-P2 threshold improvement was greater for $1000-\mathrm{Hz}$ than $4000-\mathrm{Hz}$ stimuli. It is unclear whether spectral splatter at short durations contributed to the threshold differences across frequency. The present study investigated spectro-temporal influence on N1-P2 thresholds by manipulating the stimulus rise-fall time, total duration, and stimulus type. Thresholds were estimated in three temporal conditions to compare stimuli with equal rise-fall $(2 \mathrm{~ms})$ but different total durations $(8,36 \mathrm{~ms})$, and stimuli with different rise-fall $(2,16 \mathrm{~ms})$ but equal total durations (36 $\mathrm{ms})$. Broadband noise bursts and $1000-\mathrm{Hz}$ tones were used to test each temporal condition. Preliminary results show that thresholds for both noise and tonal stimuli decrease with increasing total duration. No significant differences in threshold were observed as a function of rise-fall time nor were significant differences observed as a function of the stimulus type. The results suggest that N1-P2 thresholds are determined primarily by changes in total duration and that thresholds for short durations at $1000 \mathrm{~Hz}$ are not adversely affected by spectral splatter. [Work supported by NSF.]

2aPP29. Psychometric functions for gap detection. Mary Florentine (Commun. Res. Lab., Dept. of Speech-Lang. Pathol. and Audiol. (133FR), Northeastern Univ., Boston, MA 02115), Søren Buus, and Wei Geng (Northeastern Univ., Boston, MA 02115)

To determine the form of psychometric functions for gap detection, three normal listeners were tested at octave frequencies from 0.25 to 8 $\mathrm{kHz}$ using a constant-stimulus procedure with a cued yes-no paradigm. The gaps were carried by bandpass noises with an overall level of $85 \mathrm{~dB}$ SPL and a bandwidth equal to three auditory-filter bandwidths. To avoid audibility of spectral splatter, the bandpass noise was presented in a complementary notched-noise masker with a spectrum level $10 \mathrm{~dB}$ below that of the signal and a bandwidth equal to nine auditory-filter bandwidths. Gap durations were chosen randomly from one of six fixed values (seven at $0.25 \mathrm{kHz}$ ) ranging from 0.5 Minimal Detectable Gap durations (MDG) to 2 MDGs $(2.8$ MDGs at $0.25 \mathrm{kHz}$ ). An inaudible gap of $0.5 \mathrm{~ms}$ was included as a control condition. Although the false-alarm rate varied among the listeners, the slopes of the psychometric functions were quite similar. The slopes increased as the test frequency increased up to $2 \mathrm{kHz}$, but remained constant at the higher frequencies. These results indicate that stimulus variability is not important in determining gap-detection thresholds at $2 \mathrm{kHz}$ and above.

2aPP30. Suppression and the upward spread of masking. Andrew J. Oxenham (Northeastern Univ., 360 Huntington Ave., Boston, MA 02115, oxenham@ipo.tue.nl) and Christopher J. Plack (Sussex Univ., Brighton BN1 9QG, UK)

For signals well above a masker in frequency, signal level at threshold grows more rapidly than masker level, leading to the upward spread of masking (USM). It is unclear whether USM is due to a relative increase in masker excitation or an increase in suppression with increasing masker level. Thresholds were measured using a $4-\mathrm{kHz}$ signal, gated with a $10-\mathrm{ms}$ Hanning window. The masker was a 500-Hz-wide random Gaussian noise, centered at either $4 \mathrm{kHz}$ (on frequency) or $2.4 \mathrm{kHz}$ (off frequency) and gated with 2-ms ramps. Masker levels ranged from 50-90 dB SPL. In simultaneous-masking conditions, the signal was temporally centered in a 400-ms masker. In nonsimultaneous conditions, where no suppression should occur, the signal was in a brief gap between two 200-ms maskers. Results are qualitatively consistent with an explanation based on the characteristics of the basilar-membrane input-output function. At higher signal levels, the differential compression of masker and signal produces USM 
even in nonsimultaneous conditions (no suppression). At low signal levels (below about $40 \mathrm{~dB}$ SPL) the basilar-membrane response to single tones is nearly linear and nonlinear USM is only observed in simultaneousmasking conditions. [Work supported by the Wellcome Trust and the Royal Society.]

2aPP31. Intensity discrimination of ramped and damped tones. Robert S. Schlauch, Dennis T. Ries, Jeffrey J. DiGiovanni, Sara Elliot (Univ. of Minnesota, Minneapolis, MN 55455), and Shari L. Campbell (Univ. of Georgia, Athens, GA 30602)

Intensity discrimination of $1.0-\mathrm{kHz}$ tones that had exponentially ramped or damped envelopes was measured as a function of duration and level. Standard levels ranged from near threshold to $90 \mathrm{~dB}$ SPL and durations ranged from 10 to $200 \mathrm{~ms}$. Just-noticeable differences for some subjects were smaller for damped tones than for ramped tones for the same duration and level. For these conditions, damped tones showed more temporal integration than ramped tones. This result is somewhat surprising given that the subjective duration of ramped tones increased by a factor of 100 for stimuli ranging from 10 to $200 \mathrm{~ms}$, whereas the subjective duration of damped tones increased by a much smaller factor of 28 over the same range of durations. Possible physiological and cognitive explanations for these differences will be discussed. [Work supported by NIHNIDCD R29 DC01542.]

2aPP32. Preferential detection of rising versus falling intensity. John G. Neuhoff and Jennifer Wess (Dept. of Psych., Lafayette College, Easton, PA 18042)

An increase in intensity caused by the approach of a sound source can signal an ecological threat or opportunity, and therefore a potentially important event. A receding source produces a pattern of decreasing intensity change and a potentially less important event. Therefore preferential responding by an organism to rising intensity stimuli may provide a selective advantage. To investigate the hypothesis that listeners respond preferentially to rising intensity stimuli, listeners were presented with whitenoise samples that either rose or fell dynamically $20 \mathrm{~dB}$ (SPL) in intensity. Stimulus duration ranged from $0.5 \mathrm{~s}$ to $1.5 \mathrm{~s}$, and onset of intensity change ranged from $0 \mathrm{~s}$ to $1 \mathrm{~s}$. In a speeded $2 \mathrm{AFC}$ task listeners had to determine whether intensity rose or fell. Results show that listeners detected increases in intensity more quickly and more accurately than equivalent decreases. The findings are consistent with the interpretation that the auditory system processes some acoustic events according to a set of ecologically defined priorities.

2aPP33. Detection of differences in rate of frequency change in gliding tones. John Madden (Dept. of Commun. Sci. and Disord., Univ. of North Dakota, Grand Forks, ND 58202)

The purpose of the study was to measure discrimination for glides in frequency, based on discrimination of rate of frequency change. Changes in frequency transition span and duration were eliminated as confounding cues by using signals of equal frequency span and duration that smoothly changed in rate at the half-way point in their duration. That is, each signal consisted of a first segment at one rate of frequency change, a brief rate transition segment, and a second segment at another rate of frequency change. The listeners compared a standard signal during which there was no change in rate (a linear glide) with a target signal during which the second segment changed at a greater rate than the first. The task was to indicate which signal changed in rate. Discrimination thresholds were obtained for signals with a range of center frequencies, frequency spans, and durations. Both up- and down-glides were used. Preliminary results indicate that discrimination thresholds are relatively constant for center frequencies from $0.5 \mathrm{kHz}$ to $2 \mathrm{kHz}$, but increase by a factor of 2.5 at $6 \mathrm{kHz}$.
[Work supported by Research Grant Number 1 R15 dc 02662-01 from the National Institute on Deafness and Other Communication Disorders, National Institutes of Health.]

2aPP34. Contribution to the study of the audiometrical scores sources of 20 variation. Marielle Bruyninckx, Saefda Hamache, and Bernard Harmegnies (Dept. of Speech Commun., Univ. of Mons, 18 place du Parc B7000, Mons, Belgium, marielle.bruyninckx@umh.ac.be)

This paper summarizes the development and the presentation of a discrimination test of the relative height of two pure sounds. Sixty pairs of sounds have been presented to 68 subjects. The elaboration of these pairs was based on a selection of ten initial stimuli that were paired according to three different intervals: octave, 1/3 octave, and 11/24 octave. These stimuli were then presented by pairs to the subjects in a random way, according to an ascending or a descending sequential structure. The aim of this work is to test: whether the paired sounds' presentation order (ascending or descending) has, or has not, an effect on the subjects' results; whether the kind of frequential interval is a determining factor in the subjects' discriminatory abilities; whether the musical education plays a role on performances (in comparing musicians' and nonmusicians' performances). Together with measurement of reliability, the results show that: it is easier to discriminate sounds in an ascending structure, whatever the frequential intervals may be; there is no significant difference between $1 / 3$ octave, octave, or 11/24 octave intervals; the musicians present better discriminatory abilities than the nonmusicians concerning the ascending structure pairs, but worse abilities when the structure of the pairs is descending.

2aPP35. Hawkins and Stevens revisited at low frequencies. Adrianus J. M. Houtsma (IPO, Eindhoven Univ. of Technol., P.O. Box 513, 5600 MB Eindhoven, The Netherlands, houtsma@ipo.tue.nl)

The classical data on masking of pure tones by white noise [J. E. Hawkins, Jr. and S. S. Stevens, J. Acoust. Soc. Am. 22, 6-13 (1950)] yield a nonmonotonic critical ratio function that increases at low and at high frequencies. Modern bandwidth estimates of auditory filters, however, yield equivalent rectangular bandwidth (ERB) functions that are monotonic with frequency. This apparent discrepancy has sometimes been interpreted as evidence for frequency-dependent detection efficiency in the auditory system. Another possible reason for the discrepancy, however, could be the fact that the classical data were measured with headphones that needed substantial correction at low frequencies to compensate for acoustic leakage. To test this, the Hawkins and Stevens experiment was repeated with Etymotic ER-2 insert phones over a frequency range from 30 to $8000 \mathrm{~Hz}$. An adaptive forced choice, as well as a Bekesy tracking procedure, was used. The resulting critical ratio function shows monotonic dependence on frequency, down to $90 \mathrm{~Hz}$, similar to the ERB function. This appears to make critical ratio data, in principle, consistent with ERB data measured with notched-noise techniques, and largely eliminates the need for a frequency-dependent detection efficiency in the auditory system.

2aPP36. The perception of three tone patterns by Indian and American listeners. Nandini Iyer, Evelyn M. Hoglund, and Mari Reiss Jones (Ohio State Univ., Dept. of Speech and Hearing Sci., 51 Pressey Hall, 1070 Carmack Rd., Columbus, OH 43210)

Serial identification of three tone patterns by Indian and American listeners was investigated in three experiments. Six pitch patterns combined a high-frequency $(\mathrm{H})$ and a low-frequency tone (L) (e.g., H, H, L or $\mathrm{L}, \mathrm{L}, \mathrm{H})$. The first experiment presented all patterns at each of three different tempi (fast, medium, and slow trial blocks) with blocks presented in either ascending or descending order of tempi. Each pattern occurred six times (randomly) within a block. The second experiment investigated 
the differences in pattern perception between musicians and nonmusicians among the previously tested Indian and American listeners. In the third experiment, frequency separation between the high and low tones was investigated at two different tempi. A repeating cycle of three tone patterns was presented for identification and streaming thresholds were determined for Indian and American listeners. Preliminary results indicate that the two groups of listeners differ in their ability to identify three tone patterns. These differences were affected by the listeners' musical background. Frequency separation and tempo also differentially affected pattern perception among the two groups of listeners.

2aPP37. Tempo perception: The multiple-look strategy is limited by a short temporal window. Nathalie Panissal-Vieu and Carolyn Drake (Lab. de Psych. Exp., CNRS URA 316, Univ. Rene Descartes, 28 rue Serpente, 75006, Paris, France)

Previous studies [Drake and Botte (1993)] indicated that sensitivity to tempo changes in isochronous sequences improves as the number of intervals in the sequence increases. Further studies suggested that this multiple-look process only works within a limited temporal window of 0.5 or $2 \mathrm{~s}$ depending on the tempo of the sequence [Panissal-Vieu et al. (1995)]. This temporal window represents the time required to extract all the necessary temporal information (mean interval duration and dispersion). Two experiments investigated how this mental representation is used to make temporal judgements. In a 2 AFC paradigm, trained listeners identified the slower of two isochronous sequences. The number of intervals was varied in either the first or second sequence, with the temporal window saturated in the other sequence. The number of intervals required to saturate the temporal window of the first sequence varied with tempo, as predicted. However, only three intervals were required in the second sequence. These results suggest a buildup of a stable and accurate memory trace during the first sequence which is subsequently compared with the first two intervals of the second sequence. There is no need to recalculate the memory trace for the second sequence.

2aPP38. Investigations and analysis of assessment word for sound quality attaching importance to high-order sensation. Tomoharu Ishikawa, Shingo Fuyuki, and Makoto Miyahara (School of Information Sci., Japan Adv. Inst. of Sci. and Technol., 1-1 Asahidai, Tatsunokuchi-machi, Nomi-gun, Ishikawa-Ken, 923-12 Japan)

The standpoint of our research is to evaluate subjectively the sound quality of sound-reproducing systems by evaluating deep emotions which are brought by the sounds of music and speech. In evaluating sound qualities, assessment words that express the emotive meanings which have arisen in an evaluator's mind are used. The importance of sound reproduction is the transmission of "mind" that a musician wants to send. Therefore the assessment words which can represent the high-order sensations of the sender need to be obtained. Not only conventional assessment words but also assessment words which represent high-order sensation have been investigated and then analyzed. The method is as follows, (1) 35 representative assessment words by the KJ method from 1322 assessment words which were obtained by surveying audio publications over the past three years were obtained. (2) Psychological distances among 35 representative assessment words have been analyzed by the MDS method, and four clusters by hierarchical clustering have been obtained. (3) Independent to (2) above, representative assessment words by the public attention evaluation method have been scored and ranked. (4) The important assessment words which are strongly correlated with the overall sound quality have been selected.
2aPP39. Factor analysis of the perception of the evaluation of environmental sounds using adjectives describing sound quality, emotional state, and information carried by sounds. Toshio Sone, Kôji Abe, Kenji Ozawa, and Yôiti Suzuki (Res. Inst. of Elec. Comm., Tohoku Univ., Sendai-shi, 980-0812 Japan)

In order to investigate into the perception of environmental sounds, a psychoacoustic experiment was carried out. The experiment was performed with the SD (semantic differential) method using 66 environmental sounds for 39 pairs of the descriptive adjectives in Japanese. These pairs of descriptive adjectives are roughly divided into three groups. One of them was adjectives describing sound quality that had been used in similar studies reported so far. The other groups were adjectives describing emotional states of evaluators due to the sounds and information carried by sounds in addition to them. The new adjectives were selected for explaining a more general state of perception of the environmental sounds. As the result of a factor analysis, five factors were obtained. Three of them were factors describing "aesthetic state," "volume," and "brightness" of sounds. These factors correspond to the three well-known factors describing sound quality. Other than these, two new factors were derived: a factor concerning information on environment and a factor concerning the significance of sound. Moreover, it was shown that emotional states produced by environmental sounds correlate with the factor concerning the aesthetic state.

2aPP40. Sound quality estimation using hearing sensation. SoonHyob Kim (Kwang Woon Univ., Wolgaedong, Nowongu, Seoul, Korea, inphase@chollian.dacom.co.kr)

This paper is on a study of objective sound quality estimation by using human hearing sensation. Until now, the sound quality estimation has been done by subjective methods by people. Unfortunately, it depends on environment and condition of the human ear. So it was difficult to tell exactly how sound quality was. This study was performed to make objective estimation of sounds, regardless of the factors. In this paper, six parameters were defined, which are related to the sound quality: loudness, sharpness, fluctuation strength, roughness, pleasantness, and annoyance, and signal processing methods for them were developed. In this way, a simulator was implemented that measures human hearing sensation. To prove objectiveness of the simulator, the simulator results were compared with subjective test results which were given by people. As a result, the average error rates were $8.1 \%$ in loudness, $12 \%$ in sharpness, $20 \%$ in fluctuation strength, $18.5 \%$ in roughness, $5.5 \%$ in pleasantness, and $19 \%$ in annoyance. The overall average error rate was $13.7 \%$, which showed that the simulator is very useful.

2aPP41. Analysis of annoyance of low-frequency beat inside a vehicle. Klaus Genuit (HEAD acoustics GmbH, Ebertstrasse 30a, 52134 Herzogenrath, Germany)

Because station wagons have a greater volume than sedan vehicles, low-frequency acoustic effects ("humming") very often occur in the frequency range between 30 and $50 \mathrm{~Hz}$. This is not only unpleasant, but can lead to physiological changes in the driver due to specific properties of the acoustic signals. The mechanisms may be very varied: Excitation frequencies can be produced by the powertrain, exhaust system, or wheel suspension and are transmitted via the mounting points. Resonances in the regarded frequency range may well be present along these transmission paths. The spectral components thus arriving at the structure are radiated into the car interior via various "loudspeakers," such as floor panels, roofs, or doors. Moreover, for particular frequencies some loudspeakers may show special resonances or the volume of the vehicle interior results in amplification. During field trials, excitation mechanisms and transmission paths were investigated using the combination of Artificial Head and multichannel measurement technology that allows subjective evaluation of various models. Playback of the Artificial Head signals, however, is not only via headphones, but via a special "sound car" which also creates low-frequency excitation of airborne and structure-borne noise. This en- 
ables a connection to be made between the frequencies at the driver's ear giving cause for complaint and the various excitation points. Moreover, the use of a two-dimensional laser vibrometer enables a connection to be made between vibrating structures and the annoying sound components a driver is hearing.

2aPP42. Spectrum contrast and noise annoyance. Anna Preis (I.I.S. Univ. of Tokyo, 7-22-1 Roppongi, Minato-ku, Tokyo, 106 Japan)

According to the computational view, the identification of a sound event by the auditory system consists of processing the information it extracts from the temporal pattern and frequency spectrum. The fine structure of the frequency spectrum facilitates, among other things, the determination of the relative amplitudes of the partials. The auditory system uses the values of these amplitudes to compute the total loudness of the sound event by summing the specific loudness of each partial across a set of critical bands. This correlates well with experimental data of perceived loudness. The present study investigates whether the auditory system makes use of other information contained in this spectrum. The tested hypothesis stated that the auditory system detects change in the relative amplitude of the partials. It was assumed that these changes can be traced in experimental data of perceived annoyance of noise. The experimental results show that the best correlation between the reported annoyance and calculated values was obtained for the average absolute value of the second derivative of the weighted specific loudness calculated as a function of the critical-band rate scale. This specific loudness was weighted by the function introduced in sharpness calculation [W. Aures, Acustica 59, 130$141(1985)]$.
2aPP43. Detection and discrimination of amplitude-modulated signals in noise. King Chung (Northwestern Univ., 2299 N. Campus Dr., Evanston, IL 60208/House Ear Inst.), Fan-Gang Zeng (House Ear Inst., Los Angeles, CA), and Beverly A. Wright (Northwestern Univ., Evanston, IL)

Comodulation masking release demonstrated improved detection of pure-tone signals in amplitude-comodulated noise bands [Hall et al., J. Acoust. Soc. Am. 76(1), 50-56 (1984)]. However, in most real-world situations, signals such as speech sounds are comodulated and noises such as cocktail party and traffic noises are not. Two experiments were designed to examine whether comodulation of signals can lead to improved signal detection in noise. Experiment I used a 2 AFC method to measure detection threshold of a 2/3-oct-spaced, five-tone complex in pink noise. The complex tones were either amplitude comodulated or randomly modulated. Results from three normal-hearing listeners showed no difference in detection threshold between amplitude-comodulated and randomly modulated conditions. Experiment II used a method of constant stimuli to measure the psychometric function of discriminating six vowel-like stimuli in pink noise. These vowels consisted of three formant-frequency sinusoids which were unmodulated or amplitude-comodulated or randomly modulated by a $125-\mathrm{Hz}$ sinusoid simulating the voice pitch. While no difference was observed between comodulated and randomly modulated conditions, the psychometric function for the nomodulation control condition was shifted by $4-6 \mathrm{~dB}$ toward lower signal-to-noise ratios. A possible explanation is that auditory fibers phase-lock to formantfrequencies of the unmodulated stimuli and to the fundamental frequency of the modulated stimuli.

TUESDAY MORNING, 23 JUNE 1998

EAST BALLROOM B (S), 9:15 A.M. TO 12:00 NOON

\title{
Session 2aSA
}

\section{Structural Acoustics and Vibration: New Challenges in Structural Acoustics}

\author{
J. Gregory McDaniel, Chair \\ Aerospace and Mechanical Engineering Department, Boston University, 110 Cummington Street, \\ Boston, Massachusetts 02215
}

Chair's Introduction-9:15

\section{Invited Papers}

9:20

2aSA1. Product design and structural acoustics. Richard H. Lyon (RH Lyon Corp, 691 Concord Ave., Cambridge, MA 02138)

If, by structural acoustics the analysis and understanding of noise and related problems through an understanding of mechanical vibration is meant, then structural acoustics has a very long history in product design through the application of isolators and structural damping to products. But the greatest amount of research and methods development has been focused on fairly large commercial and military structures like submarines, aircraft, and spacecraft. The resources for, attention to, and methods for structural acoustics that has been available in these and the automotive industries has not been present for the industries that make most of the products each of us deals with daily. Structural acoustics as applied to hairdryers, vacuum cleaners, and lawnmowers presents challenges in both understanding and cost that the technical community can respond to. For example, designers would like to predict sound from CAD drawings. Such a need can be an inspiration to some of us. This presentation will provide examples of the need and some possible responses.

\section{9:45}

2aSA2. Wind noise challenge in the automobile industry. Jen-Yuan Her (Ford Motor Co., Dearborn, MI 48121) and William B. Coney (BBN Technologies, Cambridge, MA 02138)

Aerodynamically generated noise during vehicle operations is of increased importance for automobiles as operating speeds increase and design improvements reduce noise contributions from the powertrain and road tire interaction. Wind noise reaches the interior through a variety of mechanisms, including the aeroacoustic penetration and aerodynamic excitation of vibration, and its radiation of sound from vehicle greenhouse (roof, windshield, side glasses, and backbite) and underbody. Although vehicle wind noise 
performance keeps improving, the fundamentals of wind noise generation and transmission paths still remain uncertain. Instead of relying on measurements on hardware, the recent development of the practical CAE approach, which is different from direct CFD plus FEA simulation, may shed light on solving the puzzle from the statistical energy analysis (SEA) point of view. This paper raises issues to be solved in vehicle-shape-related wind noise root-cause understanding and prevention using SEA. Wind noise mechanisms such as vehicle body wall pressure fluctuations (pseudonoise) generation, its scaling law, and interaction with body structure are examined in detail. Challenging issues such as the identification of low wave number contribution versus convective ridges of pseudonoise to structure excitation and how sound is radiated to driver's and passengers' ears are discussed.

\section{0:10}

2aSA3. Brake-noise challenge in the automobile industry. Shih-Emn Chen and Jen-Yuan Her (Ford Motor Co., Dearborn, MI 48121)

Brake noises generated during vehicle operation are caused by nonlinear structural behavior that is difficult to model analytically. Noises, predominantly contact related, falling in this group can be classified into two categories based on the frequencies at which they occur: (1) brake squeal in kHz ranges; (2) brake moan and groan in 100-300 Hz range. These noises may often be accompanied by structural instability caused by some, if not all, of the following phenomena: (1) lining deformation; (2) negative slope in frictionvelocity characteristic; and (3) stick-slip. In addition to the aforementioned phenomena, coupled resonance of brake components is also a necessary condition of the brake-noise generation. Currently, vehicle brake-noise design prevention is still based on a trial and error basis. No reliable design prevention tool is yet established. Although computer simulations were sometimes used to predict the structural instabilities, no clear understanding of the link between structural instabilities and radiated noise has been established. The major challenge to automobile noise control engineers will be to establish a noise radiation model which could identify the influence of the key design parameters on the noise level.

\section{$10: 35$}

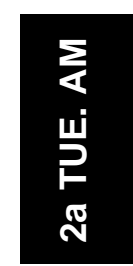

2aSA4. Integration of small transducers in commercial products. Jarmo Hietanen (Nokia Res. Ctr., P.O. Box 100, FIN-33721 Tampere, Finland, jarmo.hietanen@ research.nokia.com)

Miniaturization of electronic devices requires smaller components as well as a higher level of integration. Whenever audio acoustics is involved, as in mobile phones, it is exactly the acoustics components that offer the largest challenge in this respect. There are physical limitations to how small those components can be made, and with present methods of integration, even the smallest acceptable size is still too large. Progress in material research is expected to offer new possibilities for solving problems in further integration of components, e.g., microphones. In the development of silicon microphones, new materials and processes are utilized already. Decreasing the size of components and systems makes the problem of vibration coupling through structures more severe. Furthermore, in smaller components there are a number of physical effects to be accounted for, not only in practice but also in numerical simulation models of these systems. An example of this is the effect of viscosity when predicting acoustic performance of the system. Normally viscosity is neglected, but in very narrow ducts, for example, viscosity causes significant effects. Very small components and systems cannot be expected to operate physically, as do their larger predecessors.

\section{Contributed Papers}

\section{1:00}

2aSA5. Complex resonances of elliptic cylinders. Stéphane Ancey, Antoine Folacci, and Paul Gabrielli (URA 2053 CNRS, SDEM, Université de Corse, Faculté des Sci., BP 52, 20250 Corte, France)

Complex resonances of elliptic cylinders of arbitrary eccentricities (including the limit cases of the circular cylinder and the strip) are exactly determined by solving the Helmholtz equation with the Sommerfeld's radiation condition at infinity and various boundary conditions on the scatterer. Resonances are then fully classified along the two irreducible representations $A_{1}$ and $A_{2}$ of the symmetry group $C_{2}$ of the scatterer. This spectrum of resonances is also asymptotically recovered (i) by using the ordinary geometrical theory of diffraction, and (ii) by applying the method of the Gutzwiller-Voros spectral determinant. This last technique could be developed in acoustic multiple scattering. Indeed, in this context, because of the complexity of the system of rays and its chaotic behavior, ordinary geometrical theory of diffraction fails to provide accurate results.

\section{1:15}

2aSA6. Acoustic scattering by a three-cylinder scatterer: Theoretical and experimental study of resonances. Paul Gabrielli, Yves Decanini, Antoine Folacci, and Elisabeth Fournier (URA 2053 CNRS, SDEM, Université de Corse, Faculté des Sci., BP 52, 20250 Corte, France)

Acoustic scattering by a system of three identical parallel cylinders, located at the vertices of an equilateral triangle, is theoretically, numerically, and experimentally studied by emphasizing the role of the symmetries of the scatterer. Incident and scattered fields are expanded over the different irreducible representations of $C_{3 v}$, the symmetry group of the scatterer. From the boundary conditions, an infinite set of four linear com- plex algebraic equations is obtained, where the unknown coefficients of the scattered fields are uncoupled. As a consequence, the form function is calculated for various incidence and observation angles, and the positions of the scatterer resonances are numerically determined in the complex plane of the reduced frequency. New types of resonances appear. A physical interpretation of these resonances by the phase-matching of geometrical and surface waves along closed paths is given by using the geometrical theory of diffraction. These resonances are experimentally observed for elastic cylinders immersed in water. The work is a step toward a complete study of chaos (associated with the system of rays) in the context of multiple scattering.

\section{1:30}

2aSA7. Bandpass filters for the evaluation of short reverberation times. Malte Kob (Inst. of Tech. Acoust., RWTH Aachen, Templergraben 55, D-52056 Aachen, Germany,

kob@akustik.rwth-aachen.de)

In building acoustics, evaluating the sound insulation of building elements suffers from rather bad reproducibility. The reason is the energy flow between the building element and the surrounding structure of the test laboratory. To get more reproducible measurements, it is desirable to measure the loss factor of the element, e.g., by evaluation of the reverberation time of the structure. Unfortunately, the evaluation of decreasing reverberation times $T_{60}$ of building elements, for instance in one third octave bands is distorted by a growing influence of the applied bandpass filter. In literature a minimum permissible product of the bandwidth and the reverberation time of the filter $B \cdot T=16$ can be found but no error in the estimation of $T_{60}$ beyond this limit is given. A maximum admittable error depending on the $B \cdot T$ product is derived for several filters used in 
real time analyzers. An optimized filter according to the IEC 1260 standard is developed and results of simulated and measured short reverberation time evaluations are presented. Other possibilities of the investigation of the loss factors and their practical use are discussed.

\section{1:45}

2aSA8. Piezoelectric thin shell theoretical model and eigenfunction analysis of radially polarized ceramic cylinders. D. D. Ebenezer and Pushpa Abraham (Transducer Group, Naval Physical and Oceanograph. Lab., Naval Base, Kochi 682 004, India, root@drnpol.ren.nic.in)

A piezoelectric thin shell theoretical model of radially polarized ceramic cylinders is first developed. An eigenfunction approach is then used to determine the displacement, stress, electric field, and input electrical admittance of the cylinder. In the model, the effect of bending strain is included and it is assumed that the electric field varies linearly between the electrodes. The electrostatic condition is then used to express the electric field in terms of the displacement. Next, the equations of motion and the boundary conditions are derived by using Hamilton's variational principle. They have the same form as the corresponding equations for a thin elastic shell and are solved after expressing the frequency-dependent displacement as the sum of the static displacement and a weighted sum of the eigenfunctions of the short-circuited cylinder. The eigenfunctions are determined here by using a general method and, therefore, cylinders with arbitrary boundary conditions can be analyzed. It is shown that experimental resonance frequencies and input electrical admittance of free-free cylinders are in good agreement with those computed here and that the electric field is in good agreement with results obtained by other investigators who used a numerical method to solve the equations.

\title{
Session 2aSC
}

\section{Speech Communication, Psychological and Physiological Acoustics and Musical Acoustics: Rhythm in Music and Speech}

\author{
Robert F. Port, Cochair \\ Department Linguistics, Indiana University, 330 Memorial Hall, Bloomington, Indiana 47405 \\ J. Devin McAuley, Cochair \\ Psychology Department, University of Queensland, Brisbane, QLD, 4072 Australia
}

Chair's Introduction-8:00

Invited Papers

\begin{abstract}
8:05
2aSC1. Limit cycle dynamics in prosody. Fred Cummins (Dept. of Linguist., Northwestern Univ., Evanston, IL 60208)

Compared to music, juggling, dancing, etc., speech may seem to be only weakly rhythmic. New experimental evidence suggests, however, that rhythmic constraints on the timing of certain speech events can be quite strong. This is particularly clear when subjects repeat a phrase in time with a periodic stimulus. Mode locking, mode switching, and hysteresis have all been documented, thus showing hallmarks of the hierarchical entrainment among two periodic cycles. These cycles are coextensive with independently motivated prosodic units, the foot (or prosodic word), and the intonational phrase. It appears that this repetition task induces a second-order limit cycle dynamic in each of these units. [Work supported by the Indiana University Graduate School and the Andrew W. Mellon Foundation.]
\end{abstract}

8:25

2aSC2. Temporal correlates of foot-level timing in English and Japanese. Keiichi Tajima and Robert F. Port (Indiana Univ., 322 Memorial Hall, Bloomington, IN 47401)

\footnotetext{
Languages of the world are felt to be spoken with different kinds of rhythm. But what is the basis for this impression? Notions like "stress-timed" and "syllable-timed" have been difficult to verify experimentally because rhythm in spoken language rarely yields consistent temporal regularities. Experiments were conducted in which native English and Japanese speakers repeated phrases using a specified rhythm in time with an auditory metronome. Results demonstrate that temporal correlates exist for prosodic units approximately the size of the "foot," consisting of one relatively strong syllable followed by zero or more weak syllables. Strong (footinitial) syllables show greater temporal stability than do weak (foot-internal) syllables when the timing of syllables was perturbed by manipulating inherent segmental durations. This is found for both English and Japanese. However, in English, increasing the foot size by the addition of weak syllables is accompanied by compensatory shortening only within the foot, while in Japanese, compensation occurs both within and between adjacent feet, suggesting a weaker role for this unit. By eliciting speech in a rhythmic context, such as "speech cycling," both language-general and language-specific properties of speech rhythm can be directly measured.
} 
2aSC3. Attention, rhythmicity, and expectancy. Mari Reiss Jones (Dept. of Psych., 142 Townshend Hall, Ohio State Univ., Columbus, OH 43210)

It seems plausible that body gestures created by ourselves and other living things may derive from some underlying periodic processes. Motor rhythms are ostensibly measurable. The case for attending having a rhythmic aspect is more challenging, in part because attention is not directly observable. So the question is asked: "How does an approach that posits internal (nonobservable) oscillators actually explain attention?" Three steps are considered. The first considers that attending primarily involves selectivity. Visual attention theories often relate selectivity to space; attention is focused at one place not another. Significantly, few theories of auditory attention exist. But surely in speech and music, attention can operate selectively in time; it can be targeted to one temporal location, not another. The second step requires that nonlinear oscillators describe selective attending in time. That these models deliver is evident in several recent oscillator models, including an elegant one developed by Ed Large. Step three involves connecting oscillator activities to measurable behaviors that reflect attending. Listeners are primed with auditory patterns, only some of which display rhythms predicted to facilitate temporal expectancies accounted for by oscillator entrainment. The talk centers on these experiments, their outcomes, and implications for a theory of dynamic attending.

$$
\text { 9:05-9:20 Break }
$$

2aSC4. Context effects in time discrimination. J. Devin McAuley (Dept. of Psych., 142 Townshend Hall, Ohio State Univ., Columbus, OH 43210)

Music and speech processing exemplify skilled perception of temporal patterns, as both require an ability to make fine-grained comparisons of relative duration. Clock theories of time perception have been used to model the discrimination of isolated durations successfully, by assuming that temporal resolution is determined by the statistical variability of an independent central timing process. However, the explanatory power of the clock model has been shown to be limited when applied to more naturalistic tasks such as the detection of a temporal deviation embedded within a temporal pattern. This paper discusses an entrainment theory of time discrimination that is based on the concept of coupled oscillators. The theory accounts for a number of contextual effects in time discrimination which have been problematic for clock models. The theory also applies equally well to the discrimination of isolated durations. An important link is demonstrated between fast and slow tempos, short and long durations, and early and late onset of a comparison sequence which only becomes clear when one assumes that the relevant psychological variable in time discrimination is relative phase.

\section{9:40}

2aSC5. Categorization of temporal intervals. Edward W. Large (Dept. of Psych., Univ. of Pennsylvania, Philadelphia, PA 19104)

The rhythms of music and speech comprise sequences of temporal intervals separating the onsets of component events (e.g., notes and syllables). The perception of rhythmic patterning in such sequences involves the identification of distinct interval categories. Notating a musical rhythm, for example, relies upon the perception of nominal relationships between time intervals (e.g., 2:1, 3:1). Such categorization appears robust to perturbations that alter the absolute duration of temporal intervals. Thus it makes sense to ask whether the perception of categorical temporal relationships is "categorical perception." The answer depends not only on the ability to make category judgments, but also on the differential discriminability of temporal intervals. Entrainment models propose that perceived onset time depends upon onset phase relative to an internal oscillation [Large and Kolen, Conn. Sci. 6, 177-280 (1995); McAuley, Proc. Cog. Sci. Soc., 615-620 (1996)]. Such models predict discrimination performance, however, they do not address categorization. This paper presents an extension of entrainment models that addresses categorization: In addition to relative phase dynamics, it explicitly considers the amplitude dynamics of internal oscillations. It captures existing data on interval categorization and makes new predictions regarding categorization and discrimination performance. Tests of the predictions are discussed.

10:00

2aSC6. Models for entrainment of neural rhythms. Bill Baird (Dept. of Mathematics, Univ. of California, Berkeley, CA 94720)

Psychological results suggest that motor and perceptual behavior are organized by neural rhythms with periods from seconds to tenths of a second. It appears that their entrainment to external speech and music or to internal motor coordination rhythm is essential for effective performance. It is proposed that theta-alpha sensory-motor cycles $(5-15 \mathrm{~Hz})$ form the basis of these rhythms. They generate and entrain rhythmic motor output or temporal perceptual expectancies. It is hypothesized that cortical EEG and event-related potentials (ERPs) reflect thalamic biasing currents. The cortical evoked potential is a $5-15 \mathrm{~Hz}$ signal whose peaks and troughs correlate with stages of cortical information processing in sensory-motor tasks. Cortical rhythms are found in visual, auditory, and somatosensory areas as well as the hippocampus and reticular system that entrain to periodic inputs. If the arrival of an auditory stimulus is regular, it has been shown that $5-12 \mathrm{~Hz}$ auditory activity becomes phase locked in advance of the target stimulus to the expected arrival time. Our model of rhythmic expectation accounts for this since the theta-alpha rhythmic cycles constitute a fast clock that adjusts phase and frequency to match stimulus arrival, and longer period cycles are derived from this base clock.

10:20-10:35 Discussion 


\title{
Session 2aSP
}

\section{Signal Processing in Acoustics: Implementation Issues of Acoustical Signal Processing in Real Time Systems I}

\author{
Stergios Stergiopoulos, Chair \\ Environmental Medicine, DCIEM, Defence Civil Institute, P.O. Box 2000, North York ON M3M 3B9, Canada
}

\author{
Chair's Introduction-9:15 \\ Invited Papers
}

9:20

2aSP1. Implementation of real-time acoustic communications systems. David Herold, Robert Nation, Geoffrey Edelson, and Eric Will (Sanders, A Lockheed Martin Co., Adv. Systems Directorate, P.O. Box 868, Nashua, NH 03061-0868)

Over the past several years, the field of underwater acoustic (UWA) communications has been focusing on bandwidth efficient phase coherent modulation techniques. In this application, practical communication algorithms, and thus their implementations, are still maturing. In addition to the computer-intensive algorithms used to track and decode these acoustic data, the systems may incorporate compression, coding, and error-detection schemes to provide usable communication links. Several programs are currently underway to utilize these developments in fieldable systems. In this paper, an overview of the signal processing requirements for UWA communications is provided, as well as some constraints placed on these systems by specific applications. The effect of these requirements on overall system design and implementation is outlined.

\section{9:50}

2aSP2. An adaptive processing structure for integrated active-passive sonars deploying cylindrical arrays. Amar C. Dhanantwari (Dept. of Elec. and Comput. Eng., Univ. of Western Ontario, London, ON N6A 5B9, Canada) and Stergios Stergiopoulos (Defence and Civil Inst. of Environ. Medicine, North York, ON M3M 3B9, Canada)

The paper details the development and implementation of an adaptive processing structure for integrated active-passive sonars deploying cylindrical arrays. The main concept includes decomposition of the computationally intensive multidimensional beamformer into two simple modules, which are line and circular array beamformers. Thus, the multidimensional beamforming process can now be divided into coherent subprocesses which lead to efficient implementation in real-time sonar systems. Furthermore, the application of spatial shading to reduce the side-lobe structures can now be easily incorporated. Moreover, the new approach makes the implementation of adaptive schemes in multidimensional sensor arrays practically achievable. The proposed adaptive processing concept has been implemented in an integrated active-passive real-time sonar deploying a cylindrical array. Real data results from the adaptive and conventional beamforming outputs of the cylindrical array sonar system demonstrate the superior performance of the adaptive beamformer in suppressing the reverberation and cluttering effects in active sonar applications. Moreover, for passive sonar applications, the adaptive processing provides substantially improved angular resolution performance as compared with that of the conventional beamformer. Both these two performance improvements for a cylindrical array sonar are of particular importance for mine hunting operations.

10:20-10:35 Break

10:35

2aSP3. Sound ranging of impulsive sources in air. Brian G. Ferguson (Defence Sci. and Technol. Organisation, P.O. Box 44, Pyrmont 2009, Australia)

A passive sound ranging method based on wavefront curvature is described where the impulsive sound of a rifle firing is received at three spatially separated collinear sensors. Estimating the differential time-of-arrival (or relative time delay) of the acoustic wavefront at each pair of adjacent sensors enables the range and bearing of the source to be estimated. The temporal variability in the polar coordinates of the source is presented for real data and the system errors affecting the accuracy of the measurements are discussed. The time delay estimation technique is then applied to the passive ranging of an impulsive sound source located in the far field, where the wavefront curvature technique is no longer appropriate as the arriving wavefront is planar. For the plane-wave arrival case, the range of the sound source is estimated from the angle-of-arrival estimates at two widely spaced known locations. The source position estimated by the acoustic method is compared with the source position determined by standard surveying methods. The nonstationary behavior of the sound propagation medium leads to variability in the source localization parameters which is presented for the acoustic data collected during artillery firings. 
2aSP4. Active target detection in the ocean: Optimizing performance and cost. Zoi-Heleni Michalopoulou (Ctr. for Appl. Math. and Statistics, New Jersey Inst. of Technol., Newark, NJ 07102)

The goal of this work is to design a processor for underwater target detection with superior performance to the commonly used matched filter without the efficiency penalties of the optimal filter. The theoretically optimal filter for active detection in the ocean is a correlator between the received signal and the convolution of the transmitted signal and the impulse response of the ocean. This filter is seldom employed because of the considerable computational overhead. The practically preferred method is a standard matched filter that correlates the transmitted signal to the received signal. It is computationally efficient but analytically suboptimal because it ignores the effects of the propagation medium on the transmitted signal. The standard filter is also preferable because of its attractive property of portability across environments, this feature stemming from deliberate disregard of the physics of the propagation medium. Here, the potential for improving detection performance is explored through modified matched filters that retain the portability property but do not resort to the exhaustive multiparameter grid searches of the optimal filter. [Work supported by ONR Ocean Acoustics, through Grant No. N000149710600.]

\title{
11:35
}

2aSP5. Implementation of a broadband, environment-sensitive detector in real-time systems. Georgios Haralabus (SACLANT Undersea Res. Ctr., Viale San Bartolomeo 400, 19138 La Spezia, Italy)

Active systems, such as Low-Frequency Active Sonars (LFAS), have extensively used the matched filter method for detection purposes. This model-free technique is designed to maximize the signal-to-noise ratio of known signals irrespective of the nature of the waveguide. However, shallow water and dense multipath conditions often distort and obscure the matched filter output to a point at which reliable detection is not possible. The objective of this project is to investigate the implementation of a Channel Sensitive Processor (CSP) in real time systems. The performance improvement of the conventional matched-filter processor by exploiting characteristics of the medium is demonstrated both in an ambient noise and a reverberation-limited environment. It is clearly shown that environmental mismatch poses the primary problem in applying the CSP in real-time systems. Environmental uncertainty can be overcome by utilizing global search methods in conjunction with the CSP detector. In implementing such a scheme, the balance between potential advantages and concomitant disadvantage of inefficiently long real-time processing is examined.

TUESDAY MORNING, 23 JUNE 1998

METROPOLITAN BALLROOM (S), 9:15 A.M. TO 12:15 P.M.

Session 2aUW

Underwater Acoustics: Propagation, Ambient Noise, and Imaging (Précis Poster Session)

\author{
Evan K. Westwood, Chair \\ Applied Research Laboratories, University of Texas, EVG, P.O. Box 8029, Austin, Texas 78713-8029
}

Chair's Introduction-9:15

\section{Contributed Papers}

In this session, an oral summary of each paper will be presented followed by a poster session. All posters will be on display, and all contributors will be at their posters, from 11:10 a.m. to 12:10 p.m.

\section{9:20}

2aUW1. Comparisons of simultaneous signal reception over long range among hydrophones with unlike acoustic geometries. H. M. Walkinshaw (Box 72, Peapack, NJ 07977)

Propagation measurements were made to six hydrophones installed offshore Bermuda at depths from 430 to $2450 \mathrm{fm}$. Four were bottomed onslope or at basin depth; two were suspended, one at the sound channel axis and one $30 \mathrm{~m}$ above the deep bottom. Both impulse sources (charges detonated at 18 and $240 \mathrm{~m}$ ) and a continuous wave (cw) transducer towed at $120 \mathrm{~m}$ were used. The $800-\mathrm{nm}$ track began $40-\mathrm{nm}$ south of Bermuda at midpoint of the ensemble of hydrophones and ended off St. Thomas beyond the Puerto Rican Trench. The bottom receiver at $430 \mathrm{fm}$ had the lowest transmission loss; the deep basin hydrophone the highest. Arrival order identification from shot processing for all hydrophones showed performance depended on the degree to which the local bottom slope enabled a receiver to intercept both up and downcoming ray paths. Suspension per se was not significant. Energy partition among arrival orders indicated one arrival carried about $50 \%$ of received signal strength. About five arrivals were needed before $90 \%$ of the total signal was collected, suggesting broad ocean coverage is unavoidably linked to coping effectively with multipath propagation. [Work supported by NavElecs.]

9:25

2aUW2. The form of the normal mode that ensures escape with certainty from a surface channel. Edward R. Floyd (10 Jamaica Village Rd., Coronado, CA 92118-3208)

Surface channels trap those rays that are reflected internally before reaching a deep sound channel according to classical geometric ray tracing. In the wave representation, some leakage of trapped normal modes does occur. Recent advances in wave propagation [E. R. Floyd, Anal. Fond. L. Broglie 20, 263-279 (1995)] have shown that a wave function having subbarrier energy but with a particular compound modulation in amplitude and wave number does tunnel through a barrier with certainty and without reflection. Herein, a normal mode with a particular compound 
modulation is shown to escape from a surface channel with certainty and without reflection. These normal modes with compound modulations, which may be unfamiliar to many workers, are solutions to the wave equation. The investigation considers a bottomless sound velocity profile, $C(z)$, whose reciprocal square, $C^{-2}(z)$, forms a surface channel over the deep sound channel by two piecewise-continuous linear segments. The findings are generalized to include various surface and deep sound channels. While the quantum tunneling problem was originally solved in a trajectory representation [analogous to rigorous ray tracing, cf. E. R. Floyd, J. Acoust. Soc. Am. 80, 877-887 (1986)], herein a wave representation is used.

\section{9:30}

2aUW3. Spatial coherence of sound in convergence zones and shallow zones in the South China Sea. Dinghua Guan, Renhe Zhang, Zhenge Sun, and Yan Wang (Natl. Lab. of Acoust., Inst. of Acoust., Chinese Acad. of Sci., Beijing 100080, PROC, zrh@public.east.cn.net)

Some results of a horizontal spatial coherence experiment of sound propagation in the South China Sea were presented. Average water depth is over $4000 \mathrm{~m}$ and the depth of axis of the underwater sound channel is $1200 \mathrm{~m}$. Explosive sources were discharged at depths of 7, 100, 200, and $1000 \mathrm{~m}$. Signals were received at a horizontal receiving array of seven hydrophones with an overall length $410 \mathrm{~m}$ at a depth of $200 \mathrm{~m}$. Distances between hydrophones are 10, 20, 40, 80, 160, and $1000 \mathrm{~m}$. It is found that at the direct arrival zone and the convergence zones the sound field has good horizontal coherence. At the direct arrival zone the radius of coherence is larger than $200 \mathrm{~m}$. At the first convergence zone the radius of coherence is larger than $250 \mathrm{~m}$. At the second, third, and fourth convergence zones for frequencies lower than $1000 \mathrm{~Hz}$ the radii of coherence are larger than $250 \mathrm{~m}$. When the sound source is placed at the shadow zones, the radius of horizontal coherence is less than $100 \mathrm{~m}$, much lower than at the convergence zones. This experiment shows that a horizontal array can be used for beam forming with high spatial gain and good directivities at convergence zones.

\section{$9: 35$}

2aUW4. Pulse propagation in random media. Dalcio K. Dacol (Naval Res. Lab., Washington, DC 20375-5320)

Wave propagation in randomly fluctuating media is a problem of continuing interest in ocean acoustics. Usually the work done in this field is carried out in the frequency domain since there are powerful techniques to study wave scattering in the frequency domain, while time-domain formulations are notoriously more difficult to use and obtain results. Nevertheless, our intuition about wave phenomena is much better when discussing such phenomena in a space-time formulation than in a space-frequency formulation. In this presentation wave propagation in a weakly and randomly fluctuating medium is discussed entirely within the space-time formulation. McDonald and Kuperman derived from the wave equation an approximate time-domain progressive wave equation which is the equivalent in the space-time formulation of the well-known parabolic wave equation in the space-frequency formulation. A path integral representation for the solution of this equation is used here to study wave propagation in random media. The weakly inhomogeneity assumption yields an approximate evaluation of the path integral for the Green's function thus allowing an analysis of the time-dependent statistics of acoustic fields propagating through random media. [Work supported by ONR.]

\section{9:40}

2aUW5. Time series analysis of propagation in range-dependent shallow-water environment. Finn B. Jensen, Francesco Bini-Verona, Peter L. Nielsen (SACLANTCEN, Viale S. Bartolomeo 400, 19138 La Spezia, Italy, jensen@saclantc.nato.int), and Peter Gerstoft (UCSD, La Jolla, CA 92093-0704)

One track of the Formiche_97 shallow-water experiment in the Mediterranean involved broadband propagation over a sloping bottom with water depths varying between $120 \mathrm{~m}$ at the 16-element receive array and 600 $\mathrm{m}$ at the end of the $35-\mathrm{km}$ track. The $0.82-\mathrm{kg}$ SUS charges were detonated at two depths, 18 and $90 \mathrm{~m}$, and at range intervals of around $1.2 \mathrm{~km}$. Signals were recorded over a bandwidth of $50-5000 \mathrm{~Hz}$ on hydrophones covering the full water column. Detailed environmental measurements were also performed. Using state-of-the-art numerical modeling tools it was demonstrated the degree of accuracy with which broadband signals can be modeled in complex ocean environments, after having first finetuned the sediment parameters via a broadband acoustic inversion. The forward model used is a modified version of the ORCA code [Westwood et al., J. Acoust. Soc. Am. 100, 3631-3645 (1996)], which uses adiabatic mode theory combined with frequency-interpolation techniques to efficiently compute the broadband frequency response of the propagation channel. The broadband inversion was done using the SAGA code [Gerstoft, SACLANTCEN Rep. SM-333 (1997)], which does parameter optimization via a directed Monte Carlo search based on genetic algorithms. [Work was supported by the EC under the MAST-III project PROSIM, Contract No. MAS3-CT95-0008.]

\section{$9: 45$}

2aUW6. A two-way parabolic equation for a fluid/elastic waveguide. Joseph F. Lingevitch and Michael D. Collins (Naval Res. Lab., Washington, DC 20375, jfl@abyss.nrl.navy.mil)

The parabolic equation (PE) method is applied to acoustic propagation in a fluid above an elastic layer. Two-way PEs have previously been developed for purely fluid or elastic waveguides [M. D. Collins, J. Acoust. Soc. Am. 93, 1815-1825 (1993)]. These methods divide a rangedependent environment into a sequence of range-independent regions and apply the single scattering method to obtain the transmitted and reflected fields at each interface. Recently, these ideas have been extended to mixed media such as fluid/poro-acoustic waveguides. Improved Padé approximations for the interface conditions are useful in stabilizing the iteration formula for the transmitted and reflected fields [F. A. Milinazzo et al., J. Acoust. Soc. Am. 101, 760-766 (1997)]. In this paper, a fluid/elastic waveguide is examined and examples of the coupling between the fluid compressional wave and elastic waves at vertical interfaces are presented. [Work supported by ONR.]

\section{9:50}

2aUW7. Spatial coherence in range-dependent shallow water environments. Ilya Rozenfeld (Rensselaer Polytechnic Inst., Troy, NY 12180), William M. Carey (MIT, Cambridge, MA 02139), James F. Lynch (Woods Hole Oceanograph. Inst., Woods Hole, MA 02543), Peter Cable (BBN Systems and Technologies, New London, CT 06320), and William L. Siegmann (Rensselaer Polytechnic Inst., Troy, NY 12180)

In this presentation, effects of small random fluctuations on the spatial coherence of the sound field in shallow water with multilayered sediments will be examined. These fluctuations occur within the water volume and the sediment layers as well as at the layer interfaces. Most previous work has dealt with either range-independent or weakly range-dependent environments with random variabilities. However, strong range dependence often occurs in sound-speed profiles and at layer interfaces. For example, the region of the ACT III experimental series in the Straits of Korea shows range variability arising from sloping bathymetry and a coastal front. Consequently, spatial coherence for range-dependent waveguides with small random fluctuations will be treated. Numerical simulations of the coherence function will be presented. Results will be compared with coherence lengths of the field extracted from ACT III data.

\section{9:55}

2aUW8. Thin-sediment shear-induced effects on low-frequency broadband acoustic propagation in a shallow continental sea. Dag Tollefsen (Norwegian Defence Res. Establishment, Div. for Underwater Defence, P.O. Box 115, N-3191 Horten, Norway, dt@ffi.no)

Low-frequency acoustic broadband propagation loss data collected in areas of a shallow continental sea where the seabed is composed of hard substrates covered by thin deposits of sediment is presented. Data are 
shown to exhibit several characteristics consistent with loss due to coupling to interface waves at the sediment-substrate interface; in instances of a physically thin sediment cover, loss is attributed to guided elastic waves in the sediment [S. J. Hughes et al., J. Acoust. Soc. Am. 88, 283297 (1990)]. The interpretations are supported by numerical modeling of propagation loss using a recently developed wave number intergation code for horizontally layered fluid-solid media, and geoacoustic models consistent with geophysical data from the sites of acoustic experiments.

\section{0:00}

2aUW9. Elimination of branch cuts from the normal mode solution using gradient half-spaces. Evan K. Westwood and Robert A. Koch (Appl. Res. Labs., Univ. of Texas, P.O. Box 8029, Austin, TX 78713-8029)

A method for eliminating the branch cuts and branch line integrals from the normal mode solution to the range-invariant acoustoelastic wave equation has been developed and implemented. In the usual normal mode formulation, evaluation of the vertical wave number in the ocean waveguide's lower half-space involves a square-root operation and gives rise to a branch point at the critical angle. When the Pekeris cut is chosen, energy steeper than the critical angle is represented by leaky modes, and lateral-wave energy near the critical angle is represented by the branch line integral. When a mode lies near the branch cut, the branch line integral can be significant at all ranges. Branch line integrals are cumbersome to compute, and, for range-dependent problems, cannot be included in adiabatic and coupled-mode algorithms. By inserting a (small) positive attenuation gradient in the lower half-space, the plane wave solution is replaced by a complex-valued Airy function solution, the branch point is eliminated, the Pekeris branch cut is replaced by a series of modes, and the leaky modes become bounded at infinite depth. Implementation and applications of this approach using the normal mode model ORCA are given. [Work supported by ONR 321OA.]

\section{0:05}

2aUW10. Improving a practical broadband adiabatic normal mode model by including untrapped modes. Robert A. Koch, Evan K. Westwood, Julienne E. LeMond, and David P. Knobles (Appl. Res. Labs., Univ. of Texas, P.O. Box 8029, Austin, TX 78713-8029)

An adiabatic normal mode model that neglects untrapped mode contributions will give inaccurate transmission loss predictions for environments with bottom slopes when modes transition between being trapped and untrapped. For example, initially untrapped, but strongly excited, modes that subsequently become trapped away from the source may contribute significantly to water column fields. Results of Tindle and Zhang [Oceans '93 Proceedings, I-81] show that including untrapped modes in an adiabatic description significantly improves transmission loss predictions for the ASA benchmark wedge problem. A practical implementation of an adiabatic broadband propagation model for both trapped and untrapped modes will be described. Difficulties associated with the transition region between trapped and untrapped modes near the critical angle will be discussed, and the results of inserting a gradient in the lower half-space to solve the problem will be presented. [Work supported by ONR.]

\section{0:10}

2aUW11. Parabolic equation models for poroelastic transversely isotropic sediments. Andrew J. Fredricks, William L. Siegmann (Dept. of Math Sci., Rensselaer Polytechnic Inst., Troy, NY 12180), and Michael D. Collins (Naval Res. Lab., Washington, DC 20375)

Anisotropy in ocean sediments can have a significant effect on transmission loss. A parabolic equation (PE) model for problems involving transversely isotropic elastic sediments was recently developed [A. J. Fredricks et al., J. Acoust. Soc. Am. 101, 3182(A) (1997)]. Biot's theory of poroelasticity, which is appropriate for some sediments, is a generalization of elasticity that includes effects due to fluid-filled pores. Propagation models have been developed for poroelastic media, but only for the iso- tropic case. A poroelastic PE model is extended to handle transversely isotropic sediments. A description of the model will be presented along with examples to illustrate the effects of anisotropy. [Work supported by ONR.]

\section{0:15}

2aUW12. Inclusion of continuum effects in coupled-mode theory using leaky modes. David P. Knobles, Robert A. Koch, Evan K. Westwood, and Steven A. Stotts (Appl. Res. Labs., Univ. of Texas, Austin, TX 78713-8029)

A difficulty with coupled-mode algorithms for acoustic propagation in sloping, shallow-water regions is properly including the modal continuum. The disadvantage of the standard false-bottom approach is that a large number of modes are needed to represent the continuum, which makes the coupled-mode solution less practical. A leaky mode decomposition requires significantly fewer modes to represent the continuum. However, because the leaky modes are not bounded in the lower half-space, the overlap integrals involved in computing the coupling coefficients are undefined. In addition, the branch line integral, which is not included in the usual coupled-mode formalism, can become significant as modes undergo the transition between being trapped and being leaky. In this work, the approach of inserting a small attenuation gradient in the lower half-space is taken. The half-space gradient makes the mode functions have Airyfunction solutions, eliminates the branch cut, replaces it by a series of modes, and makes the leaky modes well behaved at infinite depth. Using the differential form of the coupled-mode equations, overlap integrals can be computed analytically using well-known formulas for Airy functions. The accuracy and efficiency of the approach is evaluated using the ASA benchmark wedge problem as a test case. [Work supported by ONR.]

\section{0:20}

2aUW13. A search algorithm to predict the resonance frequency of a shallow-water soliton packet. Stanley A. Chin-Bing, David B. King, Alex Warn Varnas, and Robert A. Zingarelli (Naval Res. Lab., Stennis Space Center, MS 39529-5004)

Recently a number of researchers have performed acoustic simulations of shallow-water regions that contain large amplitude internal waves (solitons). Their results have confirmed earlier findings: (a) mode conversions, due to acoustic interactions with soliton packets, can produce a large loss in acoustic transmission; (b) this loss occurs within a narrow band of acoustic frequencies (about the resonance frequency); and, (c) the transmission loss is most prominent when there is strong coupling between the lower-order (water-borne) propagation modes and the higher-order, very lossy (bottom interacting) modes. Using this knowledge, development of a search algorithm has begun to predict the resonance frequency of a shallow-water soliton packet. In this proof-of-concept work, a shallowwater soliton model was used to generate replicas of the soliton fields in the Strait of Messina. The allowable acoustic mode conversions were calculated and correlated with the dominate spatial wave numbers of the soliton packet. This was used to predict the resonance frequency. The resonance frequency was later confirmed by making hundreds of acoustic runs. The search algorithm will be discussed and examples shown. [Work supported by ONR/NRL and by a Federal High Performance Computing DoD grant.]

\section{0:25}

2aUW14. Numerical model for low-frequency sound propagation in inhomogeneous waveguides. Alexander G. Voronovich (NOAA/ERL/ ETL and Univ. of Colorado/CIRES, 325 Broadway, Boulder, CO 80303, agv@etl.noaa.gov)

Sound propagation in a shallow-water environment can result in the appearance of steep enough rays. Sufficiently accurate treatment of such situations with the help of the parabolic equation (PE) method can be complicated. On the other hand, propagation of low-frequency sound in inhomogeneous waveguides can be conveniently considered in terms of local modes. An algorithm for sound field calculation in the inhomoge- 
neous waveguides was suggested recently [A. Voronovich, J. Comp. Acoust. 4, 399-423 (1996)]. This algorithm is not based on parabolic approximation, it includes backpropagating field, and for 2-D situations is principally exact. Similar to the PE method it is also based on "marching," however, in this case it proceeds in the backward direction. In contrast to other exact approaches which invoke piecewise constant ("staircase") approximation of the inhomogeneous waveguide, this algorithm takes into account the mode interaction within each horizontal step. This allows one to increase the value of the horizontal step significantly making the algorithm very efficient. Generalization of this algorithm for the case of the uneven liquid bottom is considered. It is shown that due to sharp discontinuities at the bottom a bigger number of modes should be taken into account for sufficiently accurate calculations of the field in this case.

\section{0:30}

2aUW15. Broadband propagation over randomly varying, rangedependent elastic sediments. Michael Jaye (Rensselaer Polytechnic Inst., Troy, NY 12180), Xin Tang, Mohsen Badiey (Univ. of Delaware, Newark, DE 19716), and William L. Siegmann (Rensselaer Polytechnic Inst., Troy, NY 12180)

Acoustic experiments conducted over different tracks at the Atlantic Generating Station (AGS) site have shown significant transmission loss variability. These fluctuations cannot be accounted for entirely by bathymetric and water volume variations. Consequently, as suggested by previous studies, geoacoustic variations must be responsible for significant portions of the variability. We can take advantage of the relatively extensive core data that are available at the AGS site to model both the larger-scale range dependence and the smaller-scale variability. Uncertainties in the geoacoustic fields are modeled here by varying the vertical correlation length of the core profiles which changes the coefficients of representations by empirical orthogonal functions. The stochastic field representations are used for propagation modeling with both elastic and fluid sediments. Statistics of broadband signal transmission loss fluctuations are determined in terms of geoacoustic variabilities. Sensitivities of transmission loss predictions to frequency dependence of geoacoustic parameters are examined. Simulation statistics are compared with experimental data. [Work supported by ONR.]

\section{0:35}

2aUW16. The existence and coherence of ray bundles in a refractive ocean. Ivan P. Smirnov, Alexander I. Khil'ko (Inst. of Appl. Phys., Nizhny Novgorod, 46, Ulianov, Str., 603600, Russia), and Jerald W. Caruthers (Naval Res. Lab., Stennis Space Center, MS 39529)

Due to the existence of relatively smooth local extrema in the dependencies of ray-cycle lengths on initial path angles, different kinds of weakly diverging bundles (WDB) of rays can arise in refractive oceanic waveguides. As Brekhovskikh et al. have shown [Acoust. J. (in Russian) 36, 5, 824-831 (1990)], such bundles transporting high-level acoustic intensity can be interesting with respect to these applications in ocean acoustic tomography (OAT). Conditions for the existence of WDB for piecewise linear approximations of sound velocity profiles are formulated in this work. Based on these results, the possibilities for the existence of WDB for different regions of the world's oceans are discussed. Important for tomographical application, the coherence of WDB is investigated under assumptions that the acoustic signals are emitted by noise sources with limited lengths of spatial coherence and the oceanic environment is randomly inhomogeneous in spatial coherence, as described by a Gaussian distribution. Numerically, it is shown that, because within a WDB ray parameters differ only slightly, coherence in WDB is maintained for propagation to long distances. In contrast are the rays outside the WDB. The optimal space/time filtering for OAT on the basis of the developed multiscale coherence approach is discussed.
10:40

2aUW17. Vertical noise coherence measurements in shallow water using Lagrangian drifters. Fancine Desharnais (Defence Res. Establishment Atlantic, P.O. Box 1012, Dartmouth, NS B2Y 3Z7, Canada, desharnais@drea.dnd.ca), Blair R. MacDonald, and Kenneth J. Mah (Seimac, Dartmouth, NS B3B 1W6, Canada)

The vertical coherence of an acoustic noise field in shallow water (at frequencies dominated by sea surface noise) can be used to infer certain ocean bottom properties such as the compressional sound speed of the upper sediment layer. As coherence is a normalized measurement, it is independent of background noise level, or wind speed/sea state, and remains a stable measurement over a wide range of environment conditions. A set of three Lagrangian ambient noise drifters (LANDs) were developed for vertical coherence measurements. The drifters (drogued at 15-m depth) follow the near-surface currents, and take hourly measurements of coherence from their two hydrophones located at 50-m depth. The hydrophone separation is $1 \mathrm{~m}$, and the measurements are made over a usable frequency range of $100 \mathrm{~Hz}$ to $1 \mathrm{kHz}$. The drifters were deployed at Western Bank (Scotian Shelf, Canada) and drifted for up to 2.5 days. The sea conditions varied from calm to 3-4-m waves. The stability of the coherence data through the various sea states, and the impact of shipping noise on the measurements will be discussed.

\section{$10: 45$}

2aUW18. Prediction of ambient noise in the ocean, the missing components. Douglas H. Cato (Defence Sci. and Technol. Organisation, P.O. Box 44, Pyrmont, NSW 2009, Australia,

doug.cato@dsto.defence.gov.au)

The main components of ambient noise in the ocean were established by pioneers in ambient noise [V. O. Knudsen, R. S. Alford, and J. W. Emling, J. Marine Res. 7, 410-429 (1948); G. M. Wenz, J. Acoust. Soc. Am. 34, 1936-1956 (1962)] and prediction methods are generally based on the results of these studies. Most measurements were made in regions of high shipping densities where traffic noise usually obscured other components of ambient noise at low frequencies (below $200 \mathrm{~Hz}$ ), so these do not appear in most prediction methods, even though their presence was noted in the early studies. Biological noise was also recognized as important, and widely studied, but is also not usually included. Studies of ambient noise near Australia, where shipping densities are generally lower, have shown the importance of low-frequency wind-dependent noise. Biological noise is also a major component in this part of the world, often being the dominant noise in tropical waters. Biological choruses are widespread and occur with diurnal regularity. These results are not inconsistent with measurements in other parts of the world. This paper discusses important components of ambient noise that are often missing from prediction methods.

\section{0:50}

2aUW19. Application of adaptive filter in influence extraction. S. Veerabhadraiah (Naval Sci. and Technol. Lab., Ministry of Defence, Govt. of India, Viyana Nagar, Visakhapatnam-530 027, India)

The very-low-frequency ocean ambient noise changes from place to place and time to time drastically due to various factors like thermal agitation, sea state, traffic, seismic factors, and biological activity, wind speed, etc. The surface and underwater mobiles generate an influence signal within the same frequency band of ambient noise but with extremely low magnitude. The influence signal gets buried in the high ocean ambients, yielding an extremely low signal-to-noise ratio, and are as such indistinguishable in the original time traces. In certain applications, perfect localization of such mobiles is extremely essential for extracting the influence signal buried in ocean ambients. This can be done using a selfoptimizing adaptive filter known as a LMS (least mean square) filter. The filter automatically adjusts its parameters with the arrival of each new 
ambient sample with a superior convergence rate at an appropriate step size. This technique, discussed in the paper, has been developed and successfully implemented to extract the desired signal from high ambients. Some sample graphical outputs depicting a combination of signals buried in ambient and extracted signal at the filter output. Necessary theory, a flowchart for implementing the LMS algorithm, and hardware realization of the same are also included in the paper.

\section{0:55}

2aUW20. Global autofocusing techniques for acoustic imaging. Peter T. Gough and Richard L. Lane (Dept. of Elec. and Electron. Eng., Univ. of Canterbury, Private Bag 4800, Christchurch, New Zealand, gough@elec.canterbury.ac.nz)

When images of acoustic fields are computed from measured data, the quality of the image is sometimes impaired by the acoustic wavelength limits or by turbulence in the media. However, more often, the image is impaired by random displacements in the position of the detecting array. Techniques exist to optimize the image on the basis of a priori knowledge. Since this knowledge is often statistical rather than deterministic, the image computed is the most probable image given the a priori knowledge. There can be no guarantees that what is computed is the exact image since there is an infinite combination of acoustic fields and tracks able to produce the same measured field. Using examples from some simulations of an existing synthetic aperture sonar, it is shown (by appealing to Bayesian statistics) that statistical information about the towfish behavior can be used to improve the quality of the image. Inherent in this technique is a cost function to be minimized and it is the precise nature of the cost function chosen that produces the most probable image. It is techniques such as this that hold out the prospect of SAS and passive towed array sonars without the need for on-board navigation units.
11:00

2aUW21. Up-to-date state and outlook for the intensity measurement method in underwater acoustics. Vladimir Shchurov (Lab. of Ocean Acoust. Noise, Pacific Oceanological Inst., Far Eastern Branch, Russian Acad. of Sci., 43 Baltiyskaya St., Vladivostok, 690041, Russia)

The acoustic intensity measurement method consisting of four components of the acoustic field [acoustic pressure $p(t)$ and three orthogonal components of the particle velocity vector $\mathbf{V}(t)\left\{V_{x}(t), V_{y}(t), V_{z}(t)\right\}$ simultaneous measurements] did not occupy a suitable place in underwater acoustics until now. Simultaneous measurement of four components of the acoustic field enables one to construct a point receiving system possessing direct characteristics in a wide frequency range (for example, from 10 to $100 \mathrm{~Hz}$ ). By now unconventional results of investigations of underwater acoustic noises, reverberation, point source capacity, seismic noises, and underwater navigation were obtained. The intensity measurement method has given one a chance to research the acoustic field characteristics that have never been revealed during measurements of only acoustic pressure, i.e., characteristics connected with energy flux density vector. For example, a compensation of opposite energy fluxes of ambient noise and tone or signal similar to noise; horizontal energy flux of dynamic noise connected with propagational direction and the extent of wind nearsurface roughness; splitting of ambient noise total field energy density into an isotropic component connected with the isotropic (diffuse) part of the field and an anisotropic one connected with the anisotropic (coherent) part of the field related to the energy transfer in the ocean. The isotropic component (possessing zero averaged flux) existence in the field of ambient noise allows for decreasing the threshold noise level for receiving systems measuring acoustic intensity, thus enables one to research a thin structure of the acoustic field of the noise and signal. Measurements of only acoustic pressure cannot give one such information. The acoustic intensity measurement method development will allow for constructing the most up-todate acoustic devices for oceanologic processes dynamic investigation on the bases of ambient noise and sounding signal vector characteristic measurements.

TUESDAY MORNING, 23 JUNE 1998

GRAND BALLROOM III (W), 11:00 A.M. TO 12:00 NOON

\title{
Session 2aPLb
}

\section{Plenary Lecture}

\author{
Henry E. Bass, Chair \\ National Center for Physical Acoustics, University of Mississippi, University, Mississippi 38677
}

Chair's Introduction-11:00

Invited Paper

11:05

2aPLb1. Acoustics of outdoor surfaces. Keith Attenborough (Faculty of Technol., The Open Univ., Milton Keynes MK7 6AA, England)

While the development of absorbing materials for barrier surfaces is attracting considerable research interest, relatively little effort is being devoted to modifying ground surfaces for optimum ground effect. Indeed current schemes for predicting outdoor noise levels due to transportation and industry distinguish only between two types of ground; acoustically hard and acoustically soft. Ground effect phenomena are described with reference to relevant laboratory and outdoor data which indicate the influences of surface characteristics including porosity, small-scale roughness, elasticity, vegetation, extended reaction, and topography. Theories for the propagation of curved wavefronts near to porous ground and above rough ground predict the existence of surface waves. The relationship between these waves is discussed. The robustness of ground effects to meteorological influences is considered. Optimum characteristics for porous pavement with regard to propagation effects are described. Other possibilities for passive noise control reviewed include deployment of rough pavements and poroelastic materials, modifications to railway ballast, roadside cultivation, and landscaping. [Work supported by EPSRC and BBSRC (UK).] 


\title{
Session 2pPLa
}

\section{Plenary Lecture}

James E. West, Chair

Lucent Technologies, Room 2D-338, 600 Mountain Avenue, Murray Hill, New Jersey 07974

Chair's Introduction-1:00

Invited Paper

1:05

2pPLa1. Trends in electromechanical transduction. Ilene Busch-Vishniac (Mech. Eng. Dept., Univ. of Texas, Austin, TX 78712 and Aero. and Mech. Eng. Dept., Boston Univ., Boston, MA 02215)

Electromechanical transducers (sensors and actuators) are devices which link electrical and mechanical energy domains. Ideal sensors are transducers in which the state of a system is monitored without being affected. Ideal actuators are transducers in which a system state is imposed, regardless of the load imposed. In recent years there has been growing attention on electromechanical transducers for a number of reasons: they are becoming more common in consumer items instead of being limited to laboratory equipment and industrial facilities; the demands placed on transducers, particularly with regard to size, speed, cost, and sensitivity, are becoming progressively harder to meet; the applications arenas for transducers are expanding to new domains; there is a push for miniaturization of sensors and actuators; more attention is being focused on actuators, as they are very difficult to design; and the time from discovery of new materials and techniques to the application in transducers is shrinking significantly. These trends, supported with reviews of research in the area, will be examined. Repercussions of these trends, current and in the near future, will also be discussed.

\section{Session 2pAAa}

\section{Opera House Acoustics Workshop-CIARM, MCHA, and Architectural Acoustics: Opera House Design Case Histories}

\author{
Robert D. Essert, Jr., Cochair \\ Arup Acoustics, Boston House, 36-38 Fitzroy Square, London W1P 5LL, England \\ Roberto Pompoli, Cochair \\ Dipartimento di Ingegneria, via G. Saragat, 1, 44100 Ferrara, Italy \\ Chair's Introduction-1:00 \\ Invited Papers
}

1:05

2pAAa1. Acoustics in the competition for the construction of 'La Fenice" opera house. Maria Ida Biggi (Universita' Ca' Bonvicini, Venezia, Italy) and Roberto Pompoli (Dipartimento di Ingegneria-Via G. Saragat, 1, 44100 Ferrara, Italy)

In 1789, a group of nobles wished to build a new large theater in Venice. They announced a competition, in which 29 architects from all over Italy took part, presenting many different projects. Although the prize was given to one project, a different one was chosen to be executed. The author of the latter project was Giannantonio Selva and was the cause of much criticism, but it was appreciated for the results it obtained from the acoustic point of view. In his memoirs, Selva explained how he adapted the shape of the theater so that it became a semicircle with extensions on both sides and added that there were no precise theories regarding the building of a "harmonic" theater. He also appeared to be well informed on the debate about different curves to use in the hall. He suggested leaving an empty space under the orchestra pit, because "it looks good." This paper deals with the description of the acoustical requirements in the competition for the construction of "La Fenice" and with the analysis of some of the projects presented taking into account the knowledge of room acoustics in the XVIII century. 
2pAAa2. Preliminary project for the rebuilding of "LA FENICE" theater of Venice: Acoustic aspects. Mauro Strada (Istituto Architettura Universita' Venezia, 191 Santa Croce Tolentini 30135 Venezia, Italy) and Roberto Pompoli (Facolta' Ingegneria Universita' Ferrara, Via Saragat 1, 44100 Ferrara, Italy)

After a fire destroyed "La Fenice" Theater of Venice, the Town Council decided it should be rebuilt, being a place of memories for Venetians and people worldwide, committing to the Prefect, named Delegate Commissary, the management of the operation, compiling of a Regulated Contract until reconstruction is finished. He set up an expert workgroup for the drawing up of the Contract which is of main importance in the competition for judging the works. They decided to start the rebuilding of the theater as it was and where it was, preserving at least the internal and external aspects of the "SaleApollinee" halls, and the "SalaGrande" hall. They also decided to give freedom to the designers of the companies invited to the competition, concerning the stage aspects and all the facilities areas (halls for rehearsal, dressing-rooms, etc.). The problem has been the acoustic characteristics of the "Sala Grande," which had to appear aesthetically as it was, nevertheless the materials were not going to be the same as before the fire, due to newer safety fireproof requirements. This article deals with all acoustic problems and the several stages of historical-critical deepening of the acoustics of the theater before the fire, brought into effect during the compiling of the Regulated Contract.

\section{$1: 45$}

2pAAa3. The restoration of La Fenice in Venice: The consultant's viewpoint. Daniel E. Commins (commins acoust. workshop, 350 Fifth Ave., New York, NY 10118, comminsacoustics@compuserve.com)

Following the destruction by a fire of La Fenice in Venice, an extremely complex process was set in motion to attempt to rebuild the opera house as soon as possible. In this instance, acoustics is of utmost importance but it tends to be overshadowed by many other technical economical, and political requirements. Other major problems have surfaced, among which are the following: Very few opera houses, including La Fenice, are correctly documented from the point of view of acoustics; the leaders of such projects are not always aware of the impact of acoustics; the science of opera house acoustics is incomplete, to say the least. In the case of La Fenice, the decision was made to reconstruct it "as was" through a series of discussions with experts and users, and a well organized and efficient competition was set up. The present paper will attempt to analyze the strengths and weaknesses of this process, from the point of view of the "acoustics" referee.

\section{Contributed Papers}

\section{2:05}

2pAAa4. The acoustics of the Italian opera house, "Teatro di San Carlo"' in Naples, Italy. Luigi Maffei, Gino Iannace, Carmine Ianniello, and Rosario Romano (DETEC-Univ. of Naples Federico II, Piazzale Tecchio 80, 80125 Naples, Italy, maffei@unina.it)

Teatro di San Carlo is reported to be the oldest existing European opera house. This horseshoe-shaped theater was opened on the 4th of November 1737, 41 years before the opening of La Scala in Milan and 51 years before the opening of La Fenice in Venice. Surprisingly, no objective acoustical data were found in the open literature for San Carlo. Therefore, the authors were stimulated in carrying out measurements of modern objective criteria in the above-mentioned opera house. As the theater hosts both symphonic/classical and opera concerts, two sets of measurements were carried out. The first one with the stage fitted for performing symphonic and classical music, and the second one with fitted scenery and the orchestra pit open, for performing opera concerts. Measurements in the unoccupied hall, yielded now classical, objective criteria (RT, EDT, C80, G, LE, and LF) at specific locations both on the main floor and in the boxes. The sound source was located along the longitudinal axis of the stage under the proscenium arch.

\section{2:20}

2pAAa5. Acoustics of the historical opera house of Bordeaux: Comparison between the results of objective and subjective surveys. Catherine Semidor and Aline Barlet (ERIAC, Ecole d'Architecture et de paysage de Bordeaux, Domaine de Raba, F 33405 Talence Cedex, France, catherine.semidor@bordeaux.archi.fr)

This paper deals with the relationships between objective and subjective results of an acoustic survey in the historical opera house of Bordeaux. The investigations were conducted with ordinary opera-goers during four performances of "Traviata" in order to have available statistical population answers. The results of an earlier experiment [C. Semidor, Proc. IOA 19, Part 3, 29-38 (1997)] carried out in a dual-purpose hall with a test questionnaire built with simple words which explain the sen- sations of the listeners, have shown that the questions are correctly understood. The comparison of the ordinary listeners' answers with the objective results shows that the auditive sensations are fitting with the measured criteria obtained in the opera configuration with the opened pit and sceneries on the stage. For example, for the majority of people asked, the sound in this hall seems clear and coming directly from the stage (no surrounding by the sound sensation), and the sound attack impression is very clear, which is correlated by the weak values of reverberation time and EDT. In spite of comfort defects due to the age of the opera house, such as the lack of space between the seats, the architecture of the Grand Theatre of Bordeaux is very well appreciated.

\section{2:35-2:45 Break}

\section{2:45}

2pAAa6. Acoustic conditions of the San Francisco War Memorial Opera House and its renovation. Dennis Paoletti, Kurt Graffy, Larry Tedford, and Red Wetherill (Paoletti Assoc., Inc., 40 Gold St., San Francisco, CA 94133)

The War Memorial Opera House is one of the most culturally significant performing arts venues in San Francisco. The San Francisco Opera organization (which includes the performers, designers, systems, and facilities) is known worldwide for the quality of its performances. The Loma Prieta earthquake which occurred just south of San Francisco in October 1989 was the impetus for the major renovation to the War Memorial Opera House currently in progress. This paper will report on the history of the San Francisco Opera House and the current renovation. It will also touch on some of the peripheral facilities and projects resulting from the renovation. Paoletti Associates has enjoyed a long-standing professional relationship with the San Francisco Opera organization, and the San Francisco War Memorial Board of Trustees, having been involved in a number of various studies for them over the past 20 years. They recognize the importance of acoustics, and they are quite concerned about the historical and cultural reputation of their performing arts facilities. 
3:00

2pAAa7. A unique setting for an opera orchestra. Ewart A. Wetherill (Paoletti Assoc., Inc., 40 Gold St., San Francisco, CA 94133)

In 1995 the San Francisco Opera vacated the Opera House for one winter season and two summer seasons to allow for substantial renovation necessitated by damage caused during the 1989 earthquake. Rejecting the proposal of canceled opera seasons, the company tackled the more difficult option of utilizing the 4500 seat San Francisco Civic Auditorium augmented by two of San Francisco's older theaters. The cavernous and noisy Civic Auditorium, which is basically an arena with a large U-shaped balcony, presented a challenge in several dimensions. A complete stage, main floor seating, lighting, and backstage facilities had to be created. Lacking an orchestra pit, the opera designers placed the opera orchestra above dressing rooms, $16 \mathrm{ft}$ above stage level and $60 \mathrm{ft}$ behind the stage apron in a temporary orchestra enclosure. The entire undertaking is seen as a triumph of resourcefulness and willingness to face the challenge of severe physical limitations and budget constraints. Although complicated by an awkward plan configuration and limited overhead access, as well as conflicting rigging for lighting and scenery, the orchestra enclosure nevertheless evolved into a performance element that was both acoustically and visually appropriate. The development of the orchestra enclosure design is discussed in this paper.

\section{3:15}

2pAAa8. Virtual reflecting walls for improving the acoustics of defective halls. Jean-Paul Vian and Xavier Meynial (Acoust. Group, CSTB, 24 rue Joseph Fourier, F 38400 Saint-Martin d'Hères, France, vian@cstb.fr)

Many performing art centers and multipurpose halls with poor acoustics cannot be improved for economical reasons by architectural correction works or by mechanical moving devices. The principle of virtual acoustic wall [Guicking et al., J. Acoust. Soc. Am. 78 (1985)] has been successfully applied to control the reflectivity and diffusivity of concert halls existing walls. Several independent active cells composed of a microphone, an electronic unit, and a loudspeaker form a virtual wall. By appropriately controlling the open loop gain in the cells of each virtual wall it is possible to create different sound fields fully coherent in space and time with the preexisting one. The first reflections can thus be reinforced and a natural reverberation enhancement effect is obtained by the acoustic exchange between the virtual walls. Computer sound simulation of three virtual walls in a concert hall was performed and experimental results, both objective and subjective, are shown for two concert halls in which a virtual wall system was installed.

\section{3:30}

2pAAa9. The next steps: Four examples of Artec's evolving room acoustics philosophy. Russell Johnson, Robert W. Wolff, Damian J. Doria, and Ashley Goodall (Artec Consultants, Inc., 114 W. 26 St., New York, NY 10001-6812, info@artec-usa.com)

This paper examines four Artec projects that have opened in 1997, and relates them to a room acoustics philosophy that has been evolving in Artec's designs since 1970. An illustrated discussion of the Winspear Centre (Edmonton, Alberta), the Living Arts Centre in Mississauga (Ontario), the Chan Centre (Vancouver, British Columbia), and the renovations to the stage acoustics at Music Hall, Cincinnati, highlights the importance of seat-count, complete freedom from exterior noise, room shaping, and a considerable amount of adjustability in order to achieve acoustical success in performing arts facilities.

\section{$3: 45$}

2pAAa10. La Cité de la Musique in Paris. Daniel E. Commins (commins acoust. workshop, 350 Fifth Ave., New York, NY 10118, comminsacoustics@compuserve.com)

Fifteen years have been necessary to program, design, and build the Cité de la Musique in Paris. The author has been personally involved in all phases of the project and has assumed the principal acoustical responsibilities, with assistance from many acousticians. This music complex has two parts: the National Conservatory of Music and Dance, with some 250 music rooms, and the concert hall wing. The main concert hall, which has been in service for a few years, has a capacity of 900 to 1200 only, but it is designed in such a way that it can be used for various orchestral configurations: classical, in the round, central, etc. This means, of course, that acoustical reciprocity is a must: hearing conditions and musicality must be adequate for any pairs of points, in spite of the elliptical shape of the concert hall. The present paper will describe the means used to achieve a correct acoustical result in the concert hall and will give some insight into the characteristics of some of the other rooms.

\title{
Session 2pAAb
}

\section{Opera House Acoustics Workshop-CIARM, MCHA and Architectural Acoustics: Opera House Acoustics Student Design Competition}

\author{
Robert C. Coffeen, Chair \\ Architectural Engineering, University of Kansas, Kansas City, Missouri 66045
}

\begin{abstract}
The 1998 Student Design Competition involves the schematic design of a multipurpose opera hall with the design emphasizing room acoustics and noise control. Entries from college and university students interested in building acoustics will be on display from 1:00 p.m. to 4:45 p.m. on Tuesday, 23 June and from 9:15 to 5:00 p.m. on Wednesday.
\end{abstract}




\title{
Session 2pAB
}

\section{Animal Bioacoustics and Acoustical Oceanography: Fish Acoustics}

\author{
Joseph A. Clark, Chair \\ Carderock Detachment, Naval Surface Weapons Center, Code 734, Bethesda, Maryland 20085
}

\section{Invited Paper}

1:30

\begin{abstract}
2pAB1. An experimental study of the peripheral auditory mechanics in the goldfish (Carassius auratus) and oscar (Astronotus ocellatus). Mardi C. Hastings, James J. Finneran, and Corrie Derenburger (Ohio State Univ., Dept. of Mech. Eng., Columbus, OH 43210, hastings.6@osu.edu)

Dynamic mechanical responses of peripheral auditory organs in the goldfish (Carassius auratus), considered a hearing specialist because of its coupling via the Weberian ossicles between the swimbladder and inner ear, and the oscar (Astronotus ocellatus), an auditory nonspecialist, were measured in vivo using a noncontact, noninvasive ultrasonic system. Motion of the auditory organs was induced under water using pure tones at frequencies ranging from 12.5 to $3000 \mathrm{~Hz}$. Measurements were made in both species with swimbladders intact and swimbladders deflated. Results from 23 goldfish and 38 oscars show that the swimbladder controls auditory bandwidth not only in hearing specialists, but also in nonspecialists which have no special coupling between the swimbladder and inner ear. Dual resonances routinely observed in the response of the anterior swimbladder, and motion of the Weberian ossicles transmitted to the saccular otolith at higher frequencies, contribute to the relatively broad auditory bandwidth of the goldfish. Although data were recorded only in the medial-lateral direction, they clearly indicate that displacement of the saccular otolith relative to its sensory epithelium in both the goldfish and oscar correlates with auditory thresholds for these animals reported in the literature. [Work supported by ONR Grant No. N00014-94-1-0337.]
\end{abstract}

\section{Contributed Papers}

\section{1:55}

2pAB2. A cohesive lumped parameter analysis of the mechanics of the goldfish peripheral auditory system. James J. Finneran (Biosciences Div. Naval Command, Control and Ocean Surveillance Ctr., RDT\&E Div., 49620 Beluga Rd., San Diego, CA 92152-6266) and Mardi C. Hastings (Ohio State Univ., Columbus, OH 43210)

A mathematical analysis has been formulated to model the dynamic behavior of the goldfish peripheral auditory system, including the swimbladders, Weberian apparatus, and saccule. The two-chambered goldfish swimbladder is treated as a two degree-of-freedom mechanical system consisting of two coupled mass-spring-damper arrangements. The swimbladder is coupled to the Weberian ossicles using a phenomenological analysis of the anterior swimbladder tunica externa which permits both stretching and sliding. Analysis of the saccule features only a single degree-of-freedom, corresponding to the direction of orientation of the ciliary bundles. Inputs to the saccule consist of the transverse canal fluid motion and the motion of the animal's head (assumed to match the local acoustic particle motion). Mechanical properties required for the system equations were estimated from published literature and direct measurements. Results of the analysis compare favorably to experimental data for the motions of the swimbladders and Weberian ossicles. The results indivate that the Weberian apparatus has a significant impact on the hearing ability over a broad frequency range and that the saccule functions as a displacement sensor above approximately $300 \mathrm{~Hz}$. [Work supported by ONR Grant No. N00014-94-1-0337 and the ASA Hunt Fellowship.]

\section{2:15}

2pAB3. Directional acoustic startle response in the goldfish. Thomas N. Lewis and Peter H. Rogers (The George W. Woodruff School of Mech. Eng., Georgia Inst. of Technol., Atlanta, GA 30332-0405)

Fish exhibit a characteristic Mauthner-initiated startle response to sudden acoustic stimuli. This escape response is thought to be triggered by predatory attacks since an acoustic wave is launched ahead of a striking predator. The nature of acoustic stimuli which elicit the startle response was examined in free-swimming goldfish (Carassius auratus). The results showed that a threshold level of acoustic acceleration ( $\left.-30 \mathrm{~dB} r e: 1 \mathrm{~m} / \mathrm{s}^{2}\right)$, not pressure, was required to elicit a startle and that the threshold was invariant of bearing to the source. Near threshold, the fish turned in a random direction, but as the stimulus level increased, the fish was more likely to turn away from the source. The data indicated that the lateral component of the acoustic acceleration was the relevant parameter determining directionality. A proposed model uses the utricle to detect acoustic acceleration directly, the saccule to detect acoustic pressure indirectly, and logical wiring to perform the neural computation which explains the fish's behavioral performance.

\section{2:35-2:50 Break}

2pAB4. Detection of ultrasound and marine mammal echolocation clicks by the American shad (Clupeidae). David A. Mann (Tucker-Davis Technologies, Inc., 4550 N.W. 6th St., Gainesville, FL 32609), Zhongmin Lu, and Arthur N. Popper (Univ. of Maryland, College Park, MD 20742)

Clupeid fishes (herrings and shads) are among the main prey of echolocating cetaceans. Recent studies have shown that some clupeids are repelled by intense sounds from ultrasonic transducers. The hearing thresholds of a clupeid, the American shad (Alosa sapidissima) was determined using classical conditioning. Shad could detect sounds from 0.2 to $180 \mathrm{kHz}$, with two regions of best sensitivity, one from 0.2 to $0.8 \mathrm{kHz}$ and another from 25 to $150 \mathrm{kHz}$. American shad were also able to detect simulated bottlenose dolphin (Tursiops truncatus) echolocation clicks with a threshold that suggests they could detect an echolocating dolphin at distances up to $187 \mathrm{~m}$. These results have practical implications for the design of ultrasonic devices that could be used for repelling clupeids from power plant intakes and dams, as well as for the design of ultrasonic pingers that may reduce dolphin bycatch without affecting the catch of clupeids. [Work supported by NSF.] 
$\mathrm{Hz}$ ) but may influence sea noise from $100 \mathrm{~Hz}$ to $3 \mathrm{kHz}$. The highest chorus levels occur just after dusk or in the early evening, reaching up to $35 \mathrm{~dB}$

2pAB5. Morphological responses of fish to low-frequency sound. Joseph A. Clark (CDNSWC, Code 734, Bethesda, MD 20084), Jane A. Young (CDNSWC, Bethesda, MD 20084), Amrit N. Bart, and Yonathan Zohar (COMB, Baltimore, MD 21202)

Results of an earlier study [Clark et al., J. Acoust. Soc. Am. 100, 2709(A) (1996)] suggested that low-frequency vibrations could have a positive effect on the growth rate of fingerlings grown in tanks. This talk describes a more extensive study with a larger sample of fish (640 Tlapia Nilotica grown in four control tanks and four treated tanks) for a longer period of time than the previous six week period. The control tanks were mildly acoustically quieted with foam rubber tank coatings that are normally applied to reduce heat conduction into the tanks. The treated tanks were excited continuously with shakers in the frequency range of 30-60 $\mathrm{Hz}$ for the duration of the test. Average weight and length versus time for fish in treated and control tanks were measured at 3 week intervals. Differences in the two groups and their possible causes are discussed.

\section{$3: 30$}

2pAB6. Evening fish choruses near coral reef systems in the Great Barrier Reef, Australia. Robert D. McCauley (Dept. of Marine Biol., James Cook Univ., Townsville, Queensland 4811, Australia) and Douglas H. Cato (Defence Sci. Technol. Organisation, Pyrmont NSW 2009, Australia)

The nighttime ambient sea noise on and around coral reef systems in northern Australia is characterized by choruses produced by nocturnally active fishes. These choruses are produced year round, from dusk to dawn. Choruses are composed of many "pops" of between 5 and $28 \mathrm{~ms}$ length, with a decay envelope suggestive of a lightly damped swimbladder and with source levels calculated at $154-157 \mathrm{~dB}$ re: $1 \mu \mathrm{Pa}^{2}$ at $1 \mathrm{~m}$ peak to peak. Sources are found mostly concentrated over 6-20 m water depth. Choruses have a mean spectral peak at $550 \mathrm{~Hz}$ (normal range 500-750 above background levels. The recorded levels of choruses displayed distinct daily patterns, lunar patterns with higher levels around new moon periods, and seasonal patterns of highest level during the austral summer. Choruses occur over large spatial scales, with active sources heard at up to $15 \mathrm{~km}$ from reef systems and chorus influence on ambient sea noise extending considerably further. It is believed that nocturnal planktivorous fishes, such as those from the families Holocentridae and Priacanthidae, are responsible for choruses. [Work supported by the Australian Defence Science and Technology Organization.]

\section{3:50}

2pAB7. Mathematical model of echo-location of fish-eating flying bats. Anatoli Stulov (Dept. of Mech. and Appl. Math., Inst. of Cybernetics, Akadeemia tee 21, Tallinn, Estonia, EE0026)

The ability of flying bats Vespertillionidae to detect fish under the water surface using echo-location is surprised, because only onethousandth of the acoustical pulse energy penetrates into water. Supposing the fish (air bubble) reflects the whole signal backwards, the bat must detect the echo-signal which is a million times less than the transmitted one. This fantastic level of the perception sensitivity of the flying bat lies on the bound of its ability. It seems incredible that the main method of getting food is provided for by the maximum effort of bat senses. The method of the bat echo-location is explained here in terms of the interaction of the frequency-modulated acoustical pulse with the air bubble under the water surface. It was shown that in the presence of the bubble the amplitude of the echo reflected by the water surface depends on the signal frequency. In the usual range of the echo-location, $50-100 \mathrm{kHz}$ (the resonance frequency of the bubble lies near $1 \mathrm{kHz}$ ), there are at least four regions of frequency for which the amplitude of the signal reflected back to the bat varies up to $25 \%$. Thus, the bat can detect fish in water easily.

TUESDAY AFTERNOON, 23 JUNE 1998

WEST BALLROOM B (S), 2:15 TO 5:35 P.M.

\title{
Session 2pAO
}

\section{Acoustical Oceanography and Underwater Acoustics: Surf Zone Oceanography and Acoustics II}

\author{
Grant B. Deane, Chair \\ Scripps Institute of Oceanography, Marine Physical Laboratory 0238, La Jolla, California 92093-0238
}

Invited Paper

2:15

2pA01. The generation of ambient noise due to breaking surf. James H. Wilson (Dept. of Oceanogr., Naval Postgrad. School, Monterey, CA 93943, wilson@ vsinc.com), Robert H. Bourke (Naval Postgrad. School, Monterey, CA 93943), and Josette P. Fabre (Neptune Sci., Inc., Slidell, LA 70458)

The past predominance for deep-water acoustic studies has dictated that little consideration be placed on the role of the surf zone as a source of ambient noise. Recent emphasis on propagation in coastal waters has increased the awareness of the importance of breaking surf to the noise field in littoral waters. Two recent studies, in Monterey Bay, CA, and at Duck, NC, have provided for the first time information on the source level density of surf-generated ambient noise. Inverse techniques were developed to infer the source level density from the total ambient noise field as measured at several offshore hydrophones. Source level spectra from $50-1000 \mathrm{~Hz}$ demonstrate a $-5-\mathrm{dB} /$ oct slope with absolute levels varying by $10-15 \mathrm{~dB}$ dependent on wave height and distance from the surf zone. Surf noise was found to contribute $80 \%$ (95\%-99\%) of the total offshore ambient noise during light (heavy) surf conditions. The inverse technique is highly dependent on accurate modeling of the transmission loss seaward from the surf zone, being predominantly a function of the geoacoustic properties of the sea bed. Additional comments will address breaker types and factors affecting breaker height and the noise due to waves breaking on the ice edge of the Greenland Sea. 
2pAO2. Measurements of the space-time-frequency distribution of the noise in the surf zone. Richard M. Heitmeyer, Steven L. Means, Stephen C. Wales (Naval Res. Lab., Code 7121, Washington, DC 20375), Ellen S. Livingston (Office of Naval Res., Arlington, VA 22217), Jeffrey A. Schindall, and Marshall H. Orr (Naval Res. Lab., Washington, DC 20375)

The space-time-frequency distribution of the noise generated in the surf zone was measured in the SandyDuck'97 experiment during September and October 1997. The noise measurements were obtained on a 17phone, 138-m, bottom-mounted, linear array located along a line perpendicular to the shore at a depth of one to three meters. Concurrent measurements of the location, size, and time evolution of the individual breaking waves directly above the array were made using a video camera mounted on a nearby tower. The measurements of the time history of the noise at individual phones along the array indicate that in regions of wave breaking, the noise contributions are broadband bursts, isolated in time with significant variations in level. A significant number of these broadband bursts exhibit a downward frequency sweep. Similarly, measurements of the time history of the noise observed along the array at selected frequencies show significant variations in both the range extent and the propagation speeds of the noise sources. The paper will present an interpretation of this variability in terms of the space-time distribution of the breaking waves obtained from the video measurements. [Work supported by ONR.]

\section{2:50}

2pAO3. SANDYDUCK97 angle-time-frequency measurements of breaking wave noise. Ellen S. Livingston (Code 321OA, Ocean Atmosphere and Space S\&T Div., Office of Naval Res., 800 N. Quincy St., Arlington, VA 22217-5660, livinge@onr.navy.mil), Joal J. Newcomb (Naval Res. Lab., Stennis Space Center, MS 39529-5000), Richard Heitmeyer, Stephen C. Wales, and Steven L. Means (Naval Res. Lab., Washington, DC 20375-5350)

Low-frequency acoustic measurements (0 to $2400 \mathrm{~Hz})$ were taken in October 1997 as part of the SANDYDUCK97 experiment. These measurements were made on a horizontal bottom-mounted line array deployed in the surf "transition" zone at about $500 \mathrm{~m}$ offshore. The array consisted of 64 elements oriented end fire to shore in $6 \mathrm{~m}$ of water. Video cameras, mounted on a shoreside tower, monitored the breaking wave events over the transition zone array and in the nearby surf zone during data collection periods. The beamformed array data provide an angle-time-frequency distribution of the breaking wave noise as detected in the transition zone. Comparisons will be made of the angle-time-frequency characteristics from noise fields generated during low-, medium-, and high-surf conditions, with particular attention to the differences between inshore and offshore characteristics. Results from a similar data set obtained during DUCK94 will be presented for comparison as well.

\section{3:05}

2pAO4. Characteristics of the low- and high-frequency ambient sound from breaking waves in the surf zone. Ali R. Kolaini (Natl. Ctr. for Physical Acoust., Univ. of Mississippi, Coliseum Dr., University, MS 38677) and Jeffrey A. Nystuen (Univ. of Washington, Seattle, WA 98105)

An experiment was conducted along the Scripps Institution of Oceanography Pier to study the characteristics of the ambient sound in the surf zone. The role of the single large bubble's (order of a few centimeters) oscillations versus the collective oscillations of bubble clouds as possible mechanisms of generation of the low-frequency sound, especially the relatively broadband peak that appears around $20 \mathrm{~Hz}$ in the sound spectrum, is discussed. The frequency band at which ambient sound from breakers in the surf leaks out is identified. The acoustic attenuation which makes the surf zone extremely quiet above $2 \mathrm{kHz}$, and a possible method to use ambient sound to infer bubble size distribution in shallow water near the surf zone, are discussed. [Work supported by ONR.]

3:20

2pA05. A theoretical investigation of the low-frequency sound generated by breaking waves. Mark R. Loewen and Cesar E. Farell (Dept. of Mech. and Industrial Eng., Univ. of Toronto, Toronto, ON M5S 3G8, Canada, loewen@mie.utoronto.ca)

The important influence of breaking waves on the dynamics of the upper ocean has motivated research into developing techniques for quantitatively measuring the effects of wave breaking in the field. Breaking waves are a major source of sea surface sound and breaking events produce sound at frequencies as low as $10 \mathrm{~Hz}$. Collective bubble cloud oscillations have been shown to be the dominant source of the low-frequency sound. Laboratory experiments have shown that the low-frequency sound generated by breaking is correlated with the amount of wave energy dissipated and the volume of air entrained. Therefore it may be possible to use passive acoustic measurements in the field to obtain estimates of the volume of entrained air and energy dissipated by individual breaking events. Development of a model of the low-frequency sound generated by individual breaking waves is an important step in achieving this goal. A simplified model of the low-frequency sound generated by breaking has been developed based on the approach of Oguz [J. Acoust. Soc. Am. 95(4), 1895-1912 (1994)]. The importance of the simplifying assumptions used in the model and the use of the model as an aid to interpreting field and laboratory measurements will be discussed.

\section{3:35}

2pAO6. Acoustic propagation in the ocean surf zone. Steven L. Means, Richard M. Heitmeyer, Stephen C. Wales, Thomas J. Hayward (Naval Res. Lab., Code 7121, Washington, DC 20375), Ellen S. Livingston (Office of Naval Res., Arlington, VA 22217), and Jeffrey A. Schindall (Naval Res. Lab., Washington, DC 20375)

Broadband acoustic propagation measurements were conducted during the SandyDuck'97 experiment to provide a means of interpreting noise data taken both within and outside of the surf zone. The source was located outside of the nominal surf zone region at a distance of $470 \mathrm{~m}$ from the shore in $6 \mathrm{~m}$ of water. The receivers were spaced at $6-\mathrm{m}$ intervals 45 to $183 \mathrm{~m}$ offshore in 1 to $3 \mathrm{~m}$ of water. Both the source and the receivers were located along a line orthogonal to the shore. The $\mathrm{cw}$ line sets were transmitted to obtain a description of the temporal fluctuations in the narrowband propagation induced by the temporal variability of the medium, the surface, and the entrained bubble distribution, as a function of range and frequency. The $\mathrm{cw}$ pulses and biphase-modulated PR sequences were transmitted to obtain a description of the spatial and temporal variability of the multipath structure. Measurements show significant time variations even under low surf conditions. The measured results are interpreted through simulations obtained using a FEPE propagation model with nearconcurrent estimates of the bathymetry and geoacoustic parameters derived by Fabre and Wilson [IEEE J. Ocean. Eng. 22, 434-444 (1997)]. [Work supported by ONR.]

\section{3:50-4:05 Break}

4:05

2pA07. Wind-generated acoustic spectral effects in the surf zone in the presence and absence of rainfall at Duck, North Carolina. John R. Proni (NOAA/Atlantic Oceanograph. and Meteorological Lab., Ocean Acoust. Div., 4301 Rickenbacker Cswy., Miami, FL 33149, proni@aoml.noaa.gov) and John C. Wilkerson (NOAA/Natl. Environ. Satellite Data and Information Service, Washington, DC 20746)

Observations of wind-induced effects upon concurrently measured rainfall-generated spectra in the surf zone off Duck, North Carolina were made in November, 1992. Rapid changes in wind speed from about $5 \mathrm{~m} / \mathrm{s}$ to $14 \mathrm{~m} / \mathrm{s}$ were accompanied by a frequency-dependent reduction in rain- 
fall spectral level. The reduction initiated at about $50 \mathrm{kHz}$ (the highest frequency recorded) and progressed to lower frequencies with time. When the wind burst diminished, spectral levels increased. Rainfall rate was monitored using conventional rain gauges so that rainfall rate change effects could be distinguished from wind change effects in the measured acoustical spectra. The general surf-noise spectrum level changes were also observed to be affected by wind speed changes.

\section{4:20}

2pA08. Acoustic observations of suspended sediments in the Changjiang Estuary. Shuying Zhang (Shanghai Acoust. Lab., Acad. Sinica, 456 Xiao-Mu-Qiao Rd., Shanghai 200032, PROC)

An acoustic backscatter instrument of dual frequencies ( 0.5 and 1.5 $\mathrm{MHz}$ ) was developed for suspended sediment measurements and for sediment transport studies. The instrument measures the vertical concentration profiles of suspended sediments with a temporal resolution of $1 \mathrm{~s}$ and a spatial (depth) resolution of $10 \mathrm{~cm}$ for $0.5 \mathrm{MHz}$ or $2 \mathrm{~cm}$ for $1.5 \mathrm{MHz}$. The data of backscattered acoustic energy are sampled at a rate of $75 \mathrm{kHz}$ for a 450-s burst each time, so each burst comprises 450 vertical profiles. Coincidentally, six calibration points for the water column are taken through water sampling. Further data processing for each burst, such as compensation for sound transmission losses, in situ calibration of acoustic scattering intensity to real concentration of suspended sediments, and ensemble averaging over adjustable time-depth sizes permits threedimensional concentration profiles to be derived. To increase the accuracy of concentration measurement, a multisegment procedure to form an appropriate compensation and calibration curve is applied. Through observations at the Changjiang Estuary four patterns of vertical suspension profiles are identified at flood and ebb tides. In addition, lutoclines and interfacial waves within the near-bed high concentration suspensions are revealed.

\section{$4: 35$}

2pA09. Motivations for using a pulsed full spectrum Doppler to measure bedload and near-bottom suspended sediment transport. Peter Traykovski, James D. Irish, and James F. Lynch (AOP\&E Dept., WHOI, Woods Hole, MA 02543, ptraykovski@whoi.edu)

Acoustic observations of bedform migration and suspended sediment transport at the New Jersey LEO-15 site revealed that bedload and nearbottom suspended load could be the dominant sediment transport mode over wave-formed ripples on a sandy bottom. Bedforms observed during storms using a rotary sidescan sonar were found to be wave orbital scale ripples which migrated in the onshore direction, forced by asymmetrical wave orbital velocities. Estimates of suspended sand transport were calculated from observations of acoustic backscattering and water velocity profiles. Suspended sand transport was also forced by asymmetrical wave velocities, and was found to occur primarily during the weaker offshore phase of wave motion. This net offshore suspended sediment transport was an order of magnitude less than the flux associated with onshore ripple migration. Thus it is hypothesized that ripple migration is most likely forced by unobserved bedload and near-bottom suspended transport. Spatially resolving bedload transport from the stationary bed is difficult since the motion occurs within a few grain diameters of the bed. Acoustic Doppler-based techniques, which are ideal for this type of measurement since the sediment velocity can be resolved from the stationary bed in the frequency domain, are being developed.

\section{4:50}

2pA010. Changes in acoustic impedance of marine sediment covered with liquid pollutants. Henning Harms, Rainer Matuschek, and Volker Mellert (Dept. of Phys., Univ. of Oldenburg, D-26111 Oldenburg, Germany)

Acoustic properties of porous material are slightly changed by a coating layer, which is thin compared to the acoustic wavelength. These changes affect mainly the spherical wave reflection coefficient at grazing incidence. The phase change of a surface wave component is observed and enables the easy detection of the thin layer by measuring the interference of direct and guided surface waves. This method is investigated for screening observations in order to monitor liquid pollutants on marine sediment. Since sediment is usually not plain but more or less irregularly grooved, the coating by liquid pollutants will occur in patches. Laboratory experiments are carried out with rough surfaces of sediment to investigate the sensitivity of detecting an average change in impedance. The results are compared with a numerical model incorporating Biot theory [Tooms et al., J. Acoust. Soc. Am. 93, 171-181 (1998)], which was successfully applied to model experimental data of plain sediment (Harms et al., 134th meeting of ASA). Since the rough surface produces a significant backscattering, the liquid patches are detectable in the backscattered signal as well. Experimental results of this contribution are presented and the possibility of using this method for monitoring purposes discussed. [Work supported by German Ministry of Technology BMFT.]

\section{5:05}

2pAO11. Scattering by two spheres: Theory and experiment. Irina Bjørnø and Leif Bjørnø (Dept. of Industrial Acoust., Tech. Univ. of Denmark, Bldg. 425, DK2800 Lyngby, Denmark)

Extensive studies of scattering of acoustical signals by targets of different regular shapes have formed a useful background for attempts to develop procedures for remote monitoring of suspended materials in marine environments as, for instance, measurements of characteristic parameters of suspended sediments. The scattering properties of single regularshaped particles have been studied in depth by several authors in the past. However, single particle scattering cannot explain all features of scattering by suspended sediment. When the concentration of particles exceeds a certain limit, multiple particle scattering becomes important. As a first step in the investigation of mutual interactions between several particles, the acoustical scattering by two spheres has been studied theoretically and experimentally and the results are reported in this paper. The study has mainly been focused on three issues: (1) to develop a simplified theory for scattering by two elastical spheres; (2) to measure the scattering by two spheres in a water tank, and (3) to compare the theoretical/numerical results with the measured data. A number of factors influencing multiple scattering, including the geometrical distribution of particles, their shape, size, and material properties are taken into consideration in the studies to be reported. Broadband signals were used for the experimental studies.

\section{$5: 20$}

2pA012. Acoustic propagation affected by internal solitons in a coastal area. E. C. Shang, Y. Y. Wang, and L. Ostrovsky (Cooperative Inst. for Res. in Environ. Sci., Univ. of Colorado/NOAA/ETL, Boulder, CO 80303)

The NOAA/Environmental Technology Laboratory (ETL) conducted the "Coastal Ocean Probe Experiment" (COPE) in the Oregon coastal area (with water depth of $150 \mathrm{~m}$ ) in September, 1995. Strong internal soliton activities have been observed. The isotherm records measured by FLIP demonstrate that the maximum amplitude of isotherm depression can reach $\sim 30 \mathrm{~m}$. Based on these internal soliton data, numerical simulation on acoustic propagation through solitons has been conducted. Some interesting features of the acoustic field are found: (1) The internal solitons can cause significant mode coupling, but there is no abnormal transmission loss (TL) appearing in the frequency range $50-1000 \mathrm{~Hz}$. (2) Mode coupling strongly depends on frequency, mode number, and the direction of propagation. (3) The modal horizontal refraction (MHR) caused by the internal solitons is significant and offers additional useful information for acoustic monitoring of the internal soliton activities. [Work supported by NOAA.] 


\title{
Session 2pBV
}

\section{Bioresponse to Vibration/Biomedical Ultrasound and Physical Acoustics: High Intensity Therapeutic Ultrasound II}

\author{
Gail R. ter Haar, Cochair \\ Physics Department, Royal Marsden Hospital, Sutton, Surrey SM2 5PT, England \\ Pierre D. Mourad, Cochair \\ Applied Physics Laboratory, University of Washington, 1013 NE 40th Street, Seattle, Washington 98105
}

Contributed Papers

2:10

2pBV1. Optimal acoustic parameters for induced hyperthermia from focused MHz ultrasound: Phantom measurements with fluid flow and bubble activity. R. Glynn Holt and Ronald A. Roy (Dept. of Aerosp. and Mech. Eng., Boston Univ., 110 Cummington St., Boston, MA 02215, rgholt@bu.edu)

The effective coagulation of flowing blood by ultrasound, or acoustic hemostasis, is a complex process that encompasses several physical, biophysical, and biochemical facets. Motivated by this ultimate goal of efficient and predictable hemostasis, we have undertaken a series of laboratory experiments on a variety of phantoms insonified by $1-\mathrm{MHz}$ focused ultrasound at MPa amplitudes. The goal of the present study is to quantify and characterize the physical response of a viscous, particulate-laden Newtonian flow to high-intensity focused ultrasound (HIFU). Experimental hyperthermia in a tissue/flow phantom as a function of acoustic parameters (pulse width, duty cycle, amplitude), flow parameters (volume flow rate, vessel size, flow geometry), and bubble content in the flow phantom is investigated. Spatiotemporally resolved temperature measurements in the flow and surrounding tissue phantom are reported. Time permitting, visualization of the modified flow due to acoustic streaming and radiation pressure will be undertaken to correlate flow events with the hyperthermia results. [Work supported by DARPA.]

\section{2:25}

2pBV2. Numerical simulations of tissue heating created by highintensity focused ultrasound. Francesco P. Curra, Peter Kaczkowski, Pierre D. Mourad, Lawrence A. Crum (Univ. of Washington, Appl. Phys. Lab., 1013 NE 40th St., Seattle, WA 90105, pierre@apl.washington.edu), and Vera A. Khokhlova (Moscow State Univ., Moscow, Russia 119889)

Modeling of high-intensity focused ultrasound (HIFU) propagation and heating effects in tissue has usually been studied under the assumption of linear acoustics. Nevertheless, nonlinear propagation can lead to important differences in heat deposition depending on the degree of nonlinearity achieved in a HIFU field. To be presented are calculations of the spatial patterns of acoustic intensity $(I)$ and heat generation $[H=-(\partial / \partial z+1 / r \partial / \partial r) I$ for strongly nonlinear acoustic waves and $H=2 \alpha I$ for weakly nonlinear acoustic waves, where $\alpha$ is acoustic absorption] associated with focused weakly and strongly nonlinear acoustic wave propagation in biological tissue. The propagation is modeled using a KZK equation method with special attention to correct modeling of shock generation and biologically relevant attenuation, as well as using initializing data based on existing designs of transducers for acoustic hemostasis. The heat generation term forces the "bio-heat" equation, which predicts the generation, movement, and dissipation of heat within biological tissue. The tissue model contains blood flow in an artery or vein and is perfused by a capillary bed. For this talk the differences in spatial patterns of acoustic intensity created by weakly and strongly nonlinear CW $1-\mathrm{Mhz}$ acoustic wave propagation are shown. How those differences translate into differences in heat generation and therefore bioeffect within biological tissue are then demonstrated. [Work sponsored by DARPA.]

\section{2:40}

2pBV3. Occlusion of blood vessels using high-intensity focused ultrasound. Shahram Vaezy (Anesthesiology, Box 356540, Univ. of Washington, Seattle, WA 98195, adasi@u.washington.edu), Roy Martin, Peter Kaczkowski (Univ. of Washington, Seattle, WA 98195), George Keilman (Sonic Concepts, Woodinville, MA 98072), Steve Carter, Michael Caps, Pierre Mourad, and Lawrence Crum (Univ. of Washington, Seattle, WA 98195)

Vascular injury may result from both trauma and invasive medical procedures. High-intensity focused ultrasound (HIFU) was used to occlude surgically exposed blood vessels of anesthetized rabbits, as a mechanism of hemostasis applicable to vascular injury. A HIFU transducer, with a spherically curved aperture area of $6.34 \mathrm{~cm}^{2}$, a focal length of $4 \mathrm{~cm}$, and a frequency of $3.3 \mathrm{MHz}$, was used. The transducer was equipped with a conical housing filled with water, providing a coupling medium to the vessels. Femoral arteries and veins were occluded after a HIFU treatment of approximately $30-60 \mathrm{~s}$, at a focal intensity of $3000 \mathrm{~W} / \mathrm{cm}^{2}$. The vessels were severed after the treatment to confirm complete occlusion. No bleeding was observed. Gross and microscopic examination of the vessels revealed two major mechanisms of occlusion, dissection of the vessel wall and shrinkage of the vessel. In the dissection mechanism, a flap of the inner wall of the vessel peeled as a result of the HIFU treatment and caused a blockage of the blood flow, while in the shrinkage mechanism a mechanical constriction of the vessel lumen caused the blockage. HIFU may provide a valuable method for hemostasis of injured vessels by treating a region of the blood vessel, proximal to the hemorrhaging site.

\section{Invited Paper}

2pBV4. Occlusion of blood flow by high-intensity focused ultrasound. Ian Rivens, Ian Rowland, Gail ter Haar (Phys. Dept., Royal Marsden Hospital, Sutton, Surrey, UK, ian@icr.ac.uk), Mark Denbow, and Nicholas Fisk (Royal Postgraduate Med. School, Inst. of Obstetrics and Gynaecology, Queen Charlotte's and Chelsea Hospital, London, UK)

This work investigates the possiblity of occluding blood flow (in vivo) using high-intensity focused ultrasound surgery (FUS). Such an effect could be used, for example, in the noninvasive treatment of foetal dysfunctions. A high-power 1.7-MHz, 150-mm focal length, piezoelectric transducer was used to expose rat femoral vessels to a free field $I_{-}^{S P}=4660 \mathrm{~W} \mathrm{~cm}^{-2}$ for $2 \mathrm{~s}$ under terminal 
anaesthesia. Initially, eight separate exposures were used. Later experiments were aimed at determining an optimal single exposure to produce flow occlusion. Before and after exposure, magnetic resonance FISP 3-D angiograms (MRA) with submillimeter spatial resolution were acquired using a Siemens Vision MR System (1.5T) and extremity coil. In all animals, MRA showed reduced blood flow both in the treated region and distal to it. The results of single-dose optimization will be discussed. The ability to curtail blood flow using FUS allows the possibility of treating Foeto-Foetal Transfusion Syndrome by occluding the placental shunt vessels responsible for vascular inbalance in identical twins. Currently available interventional treatments (surgery or intrauterine laser therapy) have significant mortality and morbidity. The FUS has the advantage of being noninvasive. [Work was funded by the UK Medical Research Council.]

\section{Contributed Paper}

3:15

2pBV5. Measurements of sound speed in excised tissue over temperatures expected under high-intensity focused ultrasound conditions. Susannah H. Bloch, Michael R. Bailey, Lawrence A. Crum, Peter J. Kaczkowski (Appl. Phys. Lab., Univ. of Washington, Seattle, WA 98105, peter@apl.washington.edu), George W. Keilman (Sonic Concepts, Inc., Woodinville, WA 98072), and Pierre D. Mourad (Univ. of Washington, Seattle, WA 98105)

Noninvasive monitoring of high-intensity focused ultrasound (HIFU) treatment by diagnostic ultrasound is currently limited by a poor understanding of the bioacoustic effects of tissue heating. Sound-speed measurement has been proposed as a means of noninvasive thermal mapping. The sound speed in most tissues changes monotonically but nonlinearly with temperature to around $60{ }^{\circ} \mathrm{C}$. Above that temperature, at which the tissue proteins begin to denature, irreversible changes occur in the tissue and its corresponding acoustic properties. Sound-speed data around and above the range in which the tissue "cooks" is a challenge to obtain because of changes in tissue shape, compressibility, and overall dimensions associated with the cooking process. In this research, an apparatus to measure sound speed during a controlled temperature change was used to study the effects of temperature, thermal dose, and thermal dose rate on the speed of sound in various mammalian tissues. The apparatus, potential sources of error, and results will be presented, and implications for forward and inverse HIFU propagation modeling and acoustic remote sensing of HIFU-induced temperature changes will be discussed. [Work supported by DARPA.]

\section{3:30-3:45 Break}

\section{Invited Papers}

2pBV6. Role of cavitation during high intensity focused ultrasound treatment of prostate tissue. Narendra T. Sanghvi (Focus Surgery, Inc., 3940 Pendelton Way, Indianapolis, IN 46226)

The use of high intensity focused ultrasound (HIFU) has been well established to produce deep seated tissue necrosis using a short time ultrasound exposure. The scavenging system of the body replaces the necrosed tissue over time from the treated organ. However, in the case of benign prostatic hyperplasia (BPH) treatment, it is essential to remove treated tissue immediately after the treatment to offer improved urine flow rate and relief from the symptoms. Therefore, it was necessary to develop an ultrasound treatment system that not only provides thermal lesions that are deep in the tissue but also mechanical disintegration of urethra and surrounding tissue. Once the urethra is mechanically disintegrated, the necrosed tissue is sloughed with urine and provides an improved flow rate. The mechanical defect in tissue is produced using the cavitation process. The acoustic cavitation is produced by inserting a silastic catheter in the urethra. The catheter blocks the HIFU energy and produces standing waves which produce cavitation, and converts HIFU in a thermal dose larger than that produced by only absorption of acoustic energy by tissue. This method of treatment yields far greater results. The system is now being used in human BPH treatment.

2pBV7. Transrectal high-intensity ultrasound therapy of localized prostate cancer. Jean Y. Chapelon (INSERM U281, 151 Cours A. Thomas, Lyon, F69424, France, chapelon@lyon151.inserm.fr), Albert Gelet (E. Herriot Hospital, Lyon, F69434), and Emmanuel Blanc (Edap Technomed, Vaulx en Velin, F69120)

Prostate cancer is the most common cancer in men. The Ablatherm project has been developed in order to achieve a minimally invasive treatment of localized prostate cancer using transrectal high-intensity focused ultrasound (HIFU). The method uses a sharply focused wideband transducer operating in the frequency range $2.25-3.0 \mathrm{MHz}$ to induce a localized thermal necrosis with a shot of 4.5 -s exposure. This therapy transducer focused at $40 \mathrm{~mm}$ is coupled to a retractable $7.5-\mathrm{MHz}$ biplane imaging transducer. A computer-controlled positioning device moves the probe in the rectum to ablate the volume defined with the image by repeating the shots every $5 \mathrm{~s}$. For patient safety, the system includes a power monitoring circuitry combined with a real time A-mode ultrasound detection of the rectal wall. Since 1993, 44 patients have been treated in Lyon: A complete response was obtained in 26 and only 18 numerous advantages: It is minimally invasive requiring only a short hospital stay; it is repeatable unlike radiotherapy and additional treatment centered on insufficiently treated area can be performed. [Work supported by Technomed and Rhone Alpes Futur.] 
2pBV8. Concept of biological focal field and its importance in tissue resection with high-intensity focused ultrasound. Zhi B. Wang, Feng Wu, Zhi L. Wang, and Chuan Liu (Inst. of Ultrasonic Eng. in Medicine, Chongqing Medical Univ., Box 153, 1 Medical College Rd., Chongqing 400046, PROC, chhifu@public.cq.sc.cn)

Many studies showed that in vitro the size and shape of the necrosed tissue volume generated by high-intensity focused ultrasound (HIFU) were dependent upon the transducer size and radius of curvature, operating frequency, input power, pulse duration, and delay time between each pulse. In this research the shape and size of necrosed tissue volume produced by HIFU with 1.6-MHz focused transducer were carefully checked and compared in rabbits and pigs. This volume was generated by a single exposure or focal planes. It was shown that with the same therapeutic parameters, HIFU can selectively necrose one region of liver in vivo. However, its shape and size differed between in vitro and in vivo. These changes implied that the necrosed tissue volume in vivo depended upon not only HIFU conditions, but also the structure, function, and movement of target tissue. In contrast with the physical focal region, necrosed tissue volume in vivo could also be termed the biological focal field of HIFU. Theoretically, it resembled more closely the living tissue damaged by HIFU. In order to avoid the side effects, it is important to set up the data about biological focal fields of different organs in tissue resection with HIFU.

\section{4:40}

2pBV9. Changes in ultrasonic images of tissue damaged by highintensity focused ultrasound in vivo. Feng Wu, Zhi B. Wang, and Zhi L. Wang (Inst. of Ultrasonic Eng. in Medicine, Chongqing Medical Univ., Box 153, 1 Medical College Rd., Chongqing 400046, PROC, chhifu@public.cq.sc.cn)

In order to clarify the effects of high-intensity focused ultrasound (HIFU) on the liver in vivo, the morphological changes in a large animal model were detected by quantitative analysis of ultrasonic image. One region of the liver was selected as a target tissue by ultrasonic imaging. Then, using the method of focal planes, the target part of the liver was resected by ultrasound-monitored HIFU with a $1.6-\mathrm{MHz}$ extracorporeal spherical-bowl transducer. Ultrasonic images of the liver at the selective region were acquired before, immediately, and consecutively after HIFU treatment. The bit difference of ultrasonic image at the selective damage of the liver in vivo before and after HIFU treatment was detected by a quantitative analysis system. It was observed immediately after focused extracorporeal pyrotherapy. Sharp changes in ultrasonic image were also detected by consecutive monitoring after treatment. It corresponded to the histological characteristics of the changes of the damaged tissue. These results suggest that as a method to detect necrosed tissue, the quantitative analysis of ultrasonic image may also be used as an indicator of morphological changes after HIFU treatment.
2pBV10. Ultrasound-positioned high-intensity focused ultrasound in the ablation of superficial bladder wall in miniswines. Zhi B. Wang, Feng Wu, Zhi L. Wang, and Chuan Liu (Inst. of Ultrasonic Eng. in Medicine, Chongqing Medical Univ., Box 153, 1 Medical College Rd., Chongqing 400046, PROC, chhifu@public.cq.sc.cn)

In order to simulate the ablation of a superficial bladder tumor, the effectiveness of ultrasound-positioned high-intensity focused ultrasound (HIFU) on the bladder wall was determined. One part of the bladder wall in 10 miniswines was selected as a damage region using ultrasonic imaging. Then, it was treated with a $1.6-\mathrm{MHz}$ extracorporeal spherical-bowl transducer. Animals were killed immediately after treatment and the bladder wall and rectal tissue were examined macroscopically and histologically. As a result, brighter echoic regions at the selective damage of the bladder wall were detected by ultrasonic imaging immediately after treatment. These brighter echoes corresponded to the acute bladder wall damage which caused obvious necrosis on the superficial bladder wall. Also, histological analysis of the treated areas revealed epithelial denudation and irreversible changes of submucosal and superficial muscular layer under light and electron microscope. Two of the ten animals had microscopic hematuria. No macroscopic hematuria and rectal perforation occurred except for one case of a small burn on the rectal wall. It was concluded that as an extracorporeal approach HIFU can be used successfully to sonicate the selective region of the bladder wall in a large animal model in vivo.

\section{$5: 10$}

2pBV11. Effects of high-intensity focused ultrasound on $\mathbf{H}_{22}$ liver tumor: An evaluation with different therapeutic programs. Feng Wu, Zhi B. Wang, and Zhi L. Wang (Inst. of Ultrasonic Eng. in Medicine, Chongqing Medical Univ., Box 153, 1 Medical College Rd., Chongqing 400046, PROC, chhifu@public.cq.sc.cn)

Most research shows that high-intensity focused ultrasound (HIFU) offers an extracorporeal approach to local removal of tumors. In order to choose the optimal conditions for clinical trials, this study was to evaluate the effects of different therapeutic programs of HIFU with $1200 \mathrm{~W} / \mathrm{cm}^{2}$ on $\mathrm{H}_{22}$ liver tumors. Seventy-three ICR tumor-bearing mice were divided into a control group and HIFU therapeutic groups. The therapy programs were defined by the numbers of pulses and pulse durations of HIFU with a $1.6-\mathrm{MHz}$ focused transducer. The various parameters of tumor growth, $\mathrm{x}$-ray, histology, electron microscopy, and mice nutritional state were used to assess the results of HIFU treatment. The data showed that HIFU had an inhibitory effect on $\mathrm{H}_{22}$ tumor growth in all therapeutic groups. With the same input power, the local effects of tumor removal were dependent upon the pulse durations and numbers of pulse. When the total number of pulses increased within a short time, the inhibitory effect was stronger. However, the nutritional state of animals in this group became worse. It implies that severe stress response and catabolism induced by an increase of HIFU pulse in a short time would occur. 


\title{
Session 2pEA
}

\section{Engineering Acoustics: Topics in Engineering Acoustics}

\author{
P. K. Raju, Chair \\ Mechanical Engineering Department, Auburn University, Ross 201, Auburn, Alabama 36849-5341
}

\section{Contributed Papers}

\section{2:15}

2pEA1. Ultrasonic echo signals obtained in the outdoor environment. Michael J. Anderson, Jeffrey Luke, John Canning, and Dean B. Edwards (Dept. of Mech. Eng., Eng. Phys. Bldg. 324O, Moscow, ID 83844-0902)

Many, if not most, applications of ultrasonic transducers in air are for ranging systems used in a relatively structured environment. We describe the application of ultrasonic transducers to range sensing for a robotic autonomous vehicle that is intended to operate outdoors in a forested environment. Unlike the indoor environment, application of ultrasonic ranging outdoors involves a much more cluttered environment. The outdoor environment contains a large number of categories of objects and features, as well as a wide variation within each category. In this talk, a statistical summary of echoes obtained from features including ground contour, roughness elements, and obstacles common in the forest environment is presented. Information that can be extracted from the echo data that is useful to an autonomous controller is then discussed.

\section{2:30}

2pEA2. Modeling of electrostatic ultrasonic transducers with micro air-gap structure. Li-Feng Ge (Dept. of Phys., Anhui Univ., 3 feixi Rd., Helfei 230039, PROC)

A theoretical model developed by previous papers, i.e., the plate-onair-spring model or short-tube model (or DK model) [L.-F. Ge, J. Acoust. Soc. Am. 100, 2809 (1996), and Chin. Sci. Bull., Academia Sinica 42, 22 (1997)], has been successfully extended to a generalized case, in which diaphragms of transducers are loaded also by in-plane tensile forces. Based on the dynamic analysis of such an electrostatic transducer, its diaphragm can be considered as a tensile thin plate supported by airspring, or as a short tube enclosing air, terminated by a flexible tensile diaphragm and grooved backplate as a rigid wall. The physical model and mathematical formulation describing the transducer behavior has been further developed. A comparison between the tensile-plate-on-air-spring model (or TDK model) and the plate-under-tension model (may be termed TD model) [M. J. Anderson et al., J. Acoust. Soc. Am. 97, 262-272 (1995)] is discussed in the paper by an example of predicting the resonant frequencies of a U-grooved backplate transducer with $T=100 \mathrm{~N} / \mathrm{m}$ and $V p=250 \mathrm{~V}$. The fundamental frequency is estimated by the new model at $349 \mathrm{kHz}$, corresponding pistonlike motion of the diaphragm, and second at $515 \mathrm{kHz}$, corresponding to the first bending vibration mode. The predictions are more consistent with the measurements reported. [Work supported by the Anhui Education Council Foundation.]

\section{2:45}

2pEA3. Control of particles in a standing wave field using ultrasonic vibration. Shinfuku Nomura, Kouichi Murakami, Yukinori Yoshikawa, and Junji Ochi (Ehime Univ., Dept. of Mech. Eng., 3, bunkyo-cho Matuyama, 790 Japan, nomu@en1.ehime-u.ac.jp)

In order to control the motion of micromachines by acoustic power, the behavior of particles falling in several liquids with a standing wave field was studied experimentally. A PZT transducer with a frequency of 28 $\mathrm{kHz}$ was fixed to the bottom of a cylinder. Several small tubes were inserted in this cylinder, and glass particles with a diameter of about $1.5-3.5 \mathrm{~mm}$ were dropped in these tubes, respectively. Since cavitation bubbles are observed by applying ultrasonic vibration to a liquid, cavitation intensity as estimated from the erosion loss of an aluminum sheet was measured to investigate the effect of cavitation generation on the radiation pressure. Cavitation intensity in degassed water was weaker than that in ion-exchanged water; however, the decrease in velocity of the glass particles by applying ultrasonic vibration exhibited the greatest deceleration in the degassed water. Ultrasonic power effectively acts on a particle as a radiation force when cavitation does not occur and there is almost no damping of the ultrasonic wave. When varying the tube diameter, as the ratio of the diameter of tube to the particle diameter increased, so did the deceleration. All particles were levitated by the ultrasonic radiation force in a ratio of more than 0.6 .

\section{3:00}

2pEA4. Miniaturized ultrasonic sensor system for liquid analysis. Bernd Henning, Peter C. Daur, Alexander Wolf (Institut für Automation und Kommunikation e.V. Magdeburg, Steinfeldstrasse 3, D-39179 Magdeburg-Barleben, Germany, hen@ifak.fhg.de), Peter Hauptmann, and Alf Puettmer (Otto-von-Guericke Univ. IPE, Pf 4120, D-39106 Magdeburg, Germany)

At the moment, known commercial devices to measure the sound velocity in liquids are limited in their application because the relation between velocity and concentration of substances is often not clear, for instance, if it is a parabolic curve. Additionally, the temperature dependence shows similar behavior. This contribution will present an ultrasonic sensor system consisting of a miniaturized multisensor arrangement for the comprehensive acoustic characterization of liquid mixtures. The sensor is based on segmented cylindrical piezoceramics and measures velocity, impedance coefficient, attenuation coefficient, and temperature. If it is a known liquid mixture it is possible to use the measuring quantities to calculate the material properties acoustical impedance and absorption or the concentration of selected substances in complex liquids. The sensor system is able to adapt itself to different and changeable absorption conditions which is typical of industrial processes. The measuring chamber is optimized to investigate small volumes of liquid specimens. In this contribution the sensor design and the developed approach for the continuous and simultaneous measurement of acoustic properties of liquids will be described. Application examples of the sensor in the chemical and food industry will show the advantages and limits of our new sensor system.

\section{3:15}

2pEA5. The multicomponent composition of the low acoustic impedance matching layer for the ultrasonic transducer operating in the air. Tadeusz Gudra (Inst. of Telecommunication and Acoust., Wroclaw Univ. of Technol., Wybrzeze Wyspianskiego 27, 50-370 Wroclaw, Poland)

Piezoceramic transducers with matching layers are usually used to generate an ultrasonic wave in the air within the frequency range above $100 \mathrm{kHz}$. The paper presents a new material composition of low acoustic impedance named ITAKOM, whose acoustic characteristics makes it applicable as a matching medium between a piezoelectric transducer and a gas medium in a wide range of frequencies. Porosity is the common feature of all the lowest acoustic impedance materials. The results of the studies of different material compositions show that pore diameters and 
their percentage in the composite volume have greater importance than the composite make-up and render possible the elaboration of a composite material whose parameters can be defined by the percentage interdependence of components. Due to the comparatively wide range of density and wave velocity materials of acoustic impedance ranging from $0.2-1 \times 10^{6}$ $\mathrm{Pa} \cdot \mathrm{s} / \mathrm{m}$ can be produced. An example of an application of this material to construct the focusing ultrasonic transducer operating in the air at the frequency $f=1.3 \mathrm{MHz}$ in the setup of phase sensitive scanning acoustic microscopy as well as the results of object measurements are presented in the ultrasonic journal [Gudra et al., Ultrasonics 34, 711-719 (1996)].

\section{3:30}

2pEA6. High-intensity ultrasound for dewatering of slurries. Luis Elvira-Segura, German Rodriguez-Corral, Enrique Riera F. de Sarabia, and Juan A. Gallego-Juarez (Instituto de Acustica, CSIC, Serrano, 144, 28006 Madrid, Spain)

Dewatering is a process in which the water is removed from a product without changing its phase. Fine particle slurries are difficult to dewater and several processes such as filtration and centrifugation are conventionally applied. These processes, which are efficient to remove free water, are ineffective in removing water from interstitial spaces. High-intensity ultrasound can be used to assist conventional filtration processes in order to increase the rate of dewatering. Particularly, the application of ultrasonic energy has shown to be useful as a postfiltration process, once the free water has been removed. In this paper a new system is presented in which the combination of vacuum filtration and ultrasonic energy results in very efficient dewatering of very fine slurries constituted by particles sized between 0.1 and $10 \mu$. The system is based on the application of direct coupled ultrasonic energy to the cake formed on the surface of a ceramic vacuum filter by means of specially designed plate transducers. The obtained results demonstrate that the application of ultrasound significantly increases the interstitial water removal and, consequently, it diminishes the energy consumption of the whole process (dewatering and drying).

\section{3:45-4:00 Break}

\begin{abstract}
4:00
2pEA7. Parametric array in air: Distortion reduction by preprocessing. Thomas D. Kite (Dept. of Elec. and Comput. Eng., Univ. of Texas, Austin, TX 78712-1084, tom@vision.ece.utexas.edu), John T. Post (Univ. of Texas, Austin, TX 78713-8029), and Mark F. Hamilton (Univ. of Texas, Austin, TX 78712-1063)

In a parametric array, highly directional low-frequency sound is generated by the self-demodulation of an intense, amplitude-modulated highfrequency sound beam as a result of nonlinear propagation effects. The term "audio spotlight" was introduced by Yoneyama et al. [J. Acoust. Soc. Am. 73, 1532 (1983)] for a parametric array in air used to generate directional audio frequency sound with an ultrasonic primary beam. Berktay's far-field solution [Berktay, J. Sound Vib. 2, 435 (1965)] predicts a demodulated secondary waveform along the axis of the beam that is proportional to the second time derivative of the square of the modulation envelope. The secondary wave is therefore generated with high levels of harmonic distortion, even at moderate modulation indexes [Blackstock, J. Acoust. Soc. Am. 102, 3106(A) (1997)]. Integrating the modulation signal twice and taking the square root removes this distortion; however, the resulting reduction in distortion due to taking the square root is severely limited by the bandwidth of the primary beam transducer. Here, the characteristics of the transducer are taken into account in developing an appropriate predistortion scheme to achieve high quality reproduction. Relationships between distortion, modulation index, secondary bandwidth, and secondary sound-pressure level are also discussed, and results of simulations are presented. [Work supported in part by ARL:UT IR\&D.]
\end{abstract}

2pEA8. Acoustical imaging in air by a low-cost system working in the audio band. Andrea Trucco (DIBE-Univ. of Genoa, Via Opera Pia 11A, 16145 Genoa, Italy)

In order to generate a two-dimensional acoustic image of an aerial scene, a system working in the audio band and composed of low-cost conventional electroacoustic and computation devices has been developed and tested. Such a system uses a loudspeaker for the emission of acoustic pulses, eight microphones for the reception of backscattered waves, a generation/acquisition digital board, and a personal computer. As in medical echography, the adopted algorithm for the image formation is a wideband coherent beamforming followed by a scan conversion in order to display the image in a Cartesian format (as a top view of the imaged scene). Robotic vision and remote measurement are potential applications of the system developed. Two major problems that were faced are the calibration of the microphones and the study of the best configuration in terms of pulse waveform and microphone placement. A simple calibration algorithm was achieved by comparing the obtained point spread function with the theoretical expectations, whereas the best configuration was obtained by taking into account the constraints imposed by the instrumentation and the application addressed. The obtained images are of good quality, also considering the low-cost instrumentation and the highly reverberant environment in which the experiments were performed.

\section{$4: 30$}

2pEA9. Numerical analysis of high-intensity ultrasonic processing systems. Cleofe Campos-Pozuelo (Instituto de Acustica (CSIC) Serrano, 14428006 Madrid, Spain), Bertrand Dubus (Institut d'Electronique et de Microelectronique du Nord, 59046 Lille Cedex, France), and Juan A. Gallego-Juarez (Instituto de Acustica (CSIC) Serrano, 14428006 Madrid, Spain)

High-intensity applications of ultrasonic energy in industrial processing are based on the performance of the power transducers and their coupling with the processing chamber. In fact, the right distribution of the acoustic field inside the treatment chamber and the efficiency of the system transducer-chamber are determinant factors for the feasibility of the applications. Therefore, the knowledge of the influence of geometry and dimensions of the processing chamber as well as the effect of the excitation transducers on the pressure field distribution is essential for the development of practical systems. This paper deals with the finite element modeling of a high-intensity ultrasonic processing system which is composed of a flexural-plate transducer and a cylindrical tube with characteristic dimensions much longer than the wavelength. A three-dimensional numerical procedure has been developed for modeling the finite amplitude acoustic field. Fundamental and second harmonic pressure distribution are calculated for several chamber geometries and excitation conditions. It has been found that the efficiency of the system is dependent on the geometry and dimensions of the chamber as well as on the efficiency, dimensions, and vibration distribution of the exciting transducer. The results give a guideline for the design of practical processing systems.

\section{$4: 45$}

2pEA10. A method of interpolating the diffractive information of the sphere-baffled microphone in the sound field of the spherical wave. Kimitoshi Fukudome (Dept. of Acoust. Design, Kyushu Inst. of Design, Fukuoka, 815 Japan)

The sphere-baffled microphone (SBM) has been used for estimating DOA of progressive plane waves and their extractions, but it is now planned to construct the diffractive information directory (DID) of the SBM with DOA entry and source_distance entry in the sound field of spherical wave. This paper deals with the interpolation methods of getting information associated with entries not appearing in DID. Numerical computation of the diffractive information is done for the 2-element SBM (right and left microphones at both ends of a diameter of $17.68 \mathrm{~cm}$ ) at frequency points corresponding to the sampling frequency $22.05 \mathrm{kHz}$ and 1024 point DFT. Two data sets of DOA interval of $6^{\circ}[0,6,12, \ldots]$ and $[3,9,15, \ldots]$ are used for interpolation and evaluation of errors. Interpo- 
lations and their error evaluations are done in the regions of both the log magnitude versus frequency and the group delay versus frequency. Both the bilinear interpolation and cubic interpolation method result in significant interpolation errors up to $10 \mathrm{~dB}$ and $0.14 \mathrm{~ms}$ for the shadow region. Finally, a new method is presented with high accuracy of interpolation based on the landmarks-loci of the diffractive information.

\section{5:00}

2pEA11. A conceptual experiment of direct converting digital microphone. Yoshinobu Yasuno (Matsushita Commun. Industrial Co., Ltd., 600 Saedo, Tsuzukiku, Yokohama, 224 Japan) and Yasuhiro Riko (Riko Assoc. 1988-34, Kitahassaku, Midoriku, Yokohama, 226 Japan)

A transducer which directly converts signals from analog acoustic to digital electric is described. This consists of a subtracter, a sampling and holding circuit, a sigma-delta modulation circuit as a comparator, an accumulator, and a local digital-to-analog converter similar to a typical electric analog-to-digital converter. The subtracter is an electrostatic device which has a diaphragm, driving electrode, and detecting electrode. The driving electrode is divided into corresponding digital signal bits by the ratio of each area which enables one to drive the diaphragm digitally and is regarded as an electroacoustic digital-to-analog converter. The detecting electrode makes electric signals proportional to the displacement of the diaphragm driven by subtracting the incoming acoustic signal and electrically driven force. This is regarded as the subtracter. This detected signal is amplified and sampled-held and modulated by the sigma-delta procedure and generates subtracted signal to + or -1 bit which is added to the accumulator memory by a very high-speed clock rate. The output of the accumulator is the digital signal output and is also given to the driving electrodes. A 4-bit conceptual device was developed for an experiment. Some results will be reported.

\section{$5: 15$}

2pEA12. Acoustic simulation for a loudspeaker using FEM/BEM. Veijo Ikonen, Antti Suutala, and Seppo Pohjolainen (Tampere Univ. of Technol., Dept. of Math., P.O. Box 692, FIN-33101 Tampere, Finland, ikonen@alpha.cc.tut.fi)

Although the theory of acoustics is well known, computers and numerical methods have not been effectively utilized in the field. Formulating a sufficiently good physical model is often difficult. The wave equation for pressure can easily be derived, but the boundary conditions are not so obvious. The structure of the walls of an acoustic cavity and the behavior of surface materials can be quite complex. In addition, under the influence of an acoustic field the structures can start vibrating, and this vibration is then coupled to the acoustic field. Mathematically this leads to a system of two partial differential equations. This paper studies the problem of computing an internal acoustic field of a loudspeaker box. The acoustic properties of walls, made of MDF, are taken into account by using the impedance boundary condition. The coupling between the structure and the fluid is also considered. Both finite-element and boundaryelement methods (FEM/BEM with SYSNOISE) are used for numerical computations and the results are compared with each other and with analytical solutions. When the wavelength is decreased, the element grid must be refined and the number of equations becomes very large. This is why partial differential equation models are more suitable in low frequencies.

$5: 30$

2pEA13. Vibration characteristics of high frequency and complex vibration systems of $160 \mathrm{kHz}$ to $1 \mathrm{MHz}$ for ultrasonic wire bonding. Jiromaru Tsujino and Hiroyuki Yoshihara (Kanagawa Univ., Faculty of Eng., 3-27-1 Rokkakubashi, Yokohama, 221 Japan)

Vibration characteristics of high frequency and complex vibration systems of $160 \mathrm{kHz}$ to $1 \mathrm{MHz}$ for ultrasonic wire ball bonding are studied. High-frequency and complex vibrations have been shown to be very effective for ultrasonic wire bonding of IC, LSI, or various electronic devices. Required vibration displacement amplitude for successful bonding of a $600-\mathrm{kHz}$ system is about $1 / 30$ that of a conventional $60-\mathrm{kHz}$ system. For installing a conventional ceramic capillary $1.8575 \mathrm{~mm}$ in diameter required for ball bonding, longitudinal to complex transverse vibration systems $5.5-7.0 \mathrm{~mm}$ in diameter are proposed. Vibration velocity distributions along longitudinal, transverse vibration systems and ceramic capillaries and vibration loci are measured by laser Doppler vibrometers. The ceramic capillaries are measured vibrating in transverse vibration modes with one, two, or three vibration nodes along them at $160 \mathrm{kHz}$ to $1 \mathrm{MHz}$, and the conventional capillaries are revealed applicable in such highfrequency systems. The free edge of the capillary vibrates in elliptical or circular locus with the two longitudinal vibration systems driven simultaneously. Maximum vibration velocity at a capillary tip is over $0.5 \mathrm{~m} / \mathrm{s}$ (peak-to-zero value) which is enough for bonding of thin wires successfully at high frequencies.

\title{
Session 2pMU
}

\section{Musical Acoustics: Physics and Materials of Musical Instruments II}

\author{
Shigeru Yoshikawa, Chair \\ Department of Acoustical Design, Kyushu Institute of Design, 4-9-1 Shiobara, Minami-Ku, Fukuoka, 815 Japan
}

\section{Invited Papers}

\section{1:00}

2pMU1. Composite materials for musical instruments: The maturity. Charles Besnainou (Lab. d'Acoustique Musicale, Univ. Pierre et Marie Curie, 4, place Jussieu-75252 Paris Cedex 05, France)

Nowadays, musical instruments made of composite materials allow comparison with good instruments made by craftsmen. These instruments result from 20 years of accumulated experiments carried out in laboratories. The first substitute of wood was a composite of wood: plywood. Over 70 years with this new material, honorable results were obtained for an industrial line of products: piano, harp, guitar, double bass. However, composite materials made of fibers and resin are designed to imitate the structure of wood. So a specific knowledge is needed. The first attempts were on bows because it seemed easy; good results were obtained with fiberglass. The next step was to replace the neck of an electric guitar. However, it was a long time before acoustic elements such as a soundboard 
were designed. One needs first of all to develop a methodology to measure the mechanical parameters of wood: density, Young's modulus, and damping; and to cross over the mechanical results with the subjective choices of expert craftsmen. Of course, assimilated composite techniques are required. And then to learn, as in traditional apprenticeship, how to feel the quality of an instrument. Paradoxically, in terms of sound qualities, new materials need the same knowhow used by traditional instrument makers. Maturity means that the updated composite techniques are now very simple to be assimilated by craftsmen and manufacturers too; the prices of instruments are sufficiently low and the sound qualities have reached a good level. Some instruments, namely, the violin, lute, and guitar are demonstrated.

\section{$1: 25$}

2pMU2. Nonlinear effects in a vibrating string (wire). Roger J. Hanson, H. Kent Macomber, and Michael P. Kassakatis (Dept. of Phys., Univ. of Northern Iowa, Cedar Falls, IA 50614, roger.hanson@uni.edu)

Many unusual effects are observed in vibrating strings or wires as a consequence of tensional changes and longitudinal motion which yield coupled nonlinear equations for transverse motion in orthogonal directions. For a wire driven sinusoidally in a transverse direction, notable effects observed at driving frequencies near a natural resonance include the generation of motion perpendicular to the plane of the driving force, sudden jumps in amplitude and phase, whirling motion, and hysteresis [R. J. Hanson, J. M. Anderson, and H. K. Macomber, J. Acoust. Soc. Am. 96, 1549-1556 (1994)]. Further work demonstrates the generation of second, third, and higher harmonics with amplitudes sometimes comparable to the first. These harmonics, present in both the driving and perpendicular directions, contribute to a very complex total motion. All of the effects are very sensitive to asymmetries which produce slight differences in free vibration frequencies in two orthogonal directions. For the large amplitude free vibration of a plucked string, there are also significant consequences of the nonlinear effect of the tension changes. These effects for a plucked string will be related to several musical instruments. Phenomena for both the driven and plucked cases will be qualitatively demonstrated with video displays of the signals from optical motion detectors.

\section{$1: 50$}

2pMU3. On the acoustics and psychology of piano touch and tone. Anders Askenfelt (Dept. of Speech, Music and Hearing, KTH, Box 70014, S-10044, Stockholm, Sweden), Alexander Galembo, and Lola L. Cuddy (Queen's Univ., Kingston, ON K7L 3N6, Canada)

By way of "the touch," a pianist is able to achieve two degrees of freedom in the control of the key motion. Besides the final hammer velocity, the pianist can control, to some extent independently, the force history between the key and finger. By controlling the acceleration history of the key, while keeping the final hammer velocity constant, the pianist can vary the duration of the key and hammer motion and the forces between the interacting parts of the action. This will influence the intensity of vibrations in the hammer shank and of a noise precursor in the radiated sound. This report addresses the pianist's ability to change the touch parameters and to control them (at will) for musical purposes. The traditional controversy between physicists and some pianists raises a psychological question: "Why do some pianists think they are able to control a timbre of the individual tone by touch?" One hypothesis is that variations in touch change the perception of a tone for the pianist, but not for a listener, because for the pianist the tone perceived starts at finger-key contact, while for a listener it starts when the hammer hits the string.

\section{Contributed Papers}

\section{2:15}

2pMU4. Dynamic mechanical measurements on violin wood and audience evaluations of violin tone quality. Edwin R. Fitzgerald (Johns Hopkins Univ., 3400 N. Charles St., Baltimore, MD 21218), Carleen M. Hutchins, and Morton A. Hutchins (Catgut Acoust. Soc., Montclair, NJ 07042)

At the 125th Acoustical Society of America meeting in Ottawa, Canada, values of complex shear compliance $\left(J^{*}=J^{\prime}-i J^{\prime \prime}\right)$, modulus $\left(G^{*}=1 / J^{*}\right)$, and shear wave velocity and attenuation, measured over a continuous frequency range from 10 to $10000 \mathrm{~Hz}$ at $20{ }^{\circ} \mathrm{C}$ and $50 \% \mathrm{RH}$, were described for samples of spruce and maple wood used in the construction of six violins. Four wood samples were measured for each violin, viz., samples of spruce and maple with and without cross grain. Relation of the violin wood dynamic mechanical properties to plate tuning of the violins also was discussed. Following these presentations, two members of the Ottawa Symphony orchestra each played two short pieces on each of the six violins, and the audience was asked for written comments on the tone quality of each violin. The evaluations display some variations as to the violin(s) with the best tone, but one of the six was rated as good or best tone by none, and the worst tone by many. The dynamic mechanical shear properties of this violin's wood differ in several respects from those of the other violins. Similar property differences for this wood relative to the others also exist in extensional moduli determined from flexural vibrations.

\section{2:30}

2pMU5. Vibrational modes of a tenor steel pan. Uwe J. Hansen (Phys. Dept., Indiana State Univ., Terre Haute, IN 47809, phhanse@scifac.indstate.edu) and Thomas D. Rossing (Northern Illinois Univ., DeKalb, IL 60115)

The tenor or lead pan has 28 or 29 notes covering the range of $\mathrm{C}_{4}$ to $\mathrm{E}_{6}$. In spite of its being the lead voice in a typical steel band, little research has been reported previously on its modes of vibration or its acoustical behavior. Using electronic TV holography, we have studied the modes of vibration of the various note areas in tenor pans by two different makers. Note areas generally have one or two overtone modes tuned harmonically to the fundamental [Rossing et al., Phys. Today 49(3), 24-29 (1996)]. Special attention has been paid to the strong acoustical and mechanical coupling between the note areas as they vibrate.

\section{2:45-3:00 Break}

\section{3:00}

2pMU6. Observation of the brass player's lips in motion. R. Dean Ayers (Dept. of Phys. and Astron., California State Univ., Long Beach, 1250 Bellflower Blvd., Long Beach, CA 90840, rdayers@csulb.edu)

Mouthpieces with flat windows are used to examine the lip reed's motion during the steady parts of notes played on brass instruments. Magnified stroboscopic images show different parts of the upper lip moving 
out of phase with each other. Sighting directly along the channel between the lips, one can observe a Rayleigh wave propagating downstream in the flesh of the upper lip. Downward crests of this disturbance reach maximum amplitude at the downstream end of the channel, where they provide most of the valving action on the air flow. The lower lip moves more as a lumped element, and mostly in the longitudinal direction. A computer model for this behavior will be complicated and may not show much improvement over the two-dimensional model of Adachi and Sato [J. Acoust. Soc. Am. 99, 1200-1209 (1996)]. Of potentially greater value is a more accurate mental image for performers. Muscles controlling the embouchure just establish the boundary conditions for the motion of a thin, passive layer of flesh. This knowledge may help beginners to avoid such practices as jamming the mouthpiece against the lips and tensing muscles against each other unnecessarily.

\section{$3: 15$}

2pMU7. Number of degrees of freedom of a lip-reed in brassinstrument playing. Xavier Boutillon (Dept. of Phys., Univ. of Michigan, Ann Arbor, MI 48109-1120)

A lip-reed is a double continuous system that potentially exhibits several degrees of freedom. Numerical simulations for sound synthesis as well as fitting a model with measurements naturally improve while increasing the number of degrees of freedom that are put into the model. However, this parameter is worth investigating as such. In a first approach, lips are coupled to a Helmholtz resonator whose resonance factor is actively reinforced, as described in Chen and Weinreich ["Nature of lip reed,' J. Acoust. Soc. Am. 99, 1227-1233 (1996)]. The dimensionality of the attractor or permanent regime of this nonlinear dynamical system is computed with embedding techniques of the corresponding time-series and information-based measurements [Gershenfeld et al., Time Series Prediction (Addison-Wesley, Reading, MA, 1994)]. Preliminary results indicate a dimension of 1,2 or 3 for different couplings between the lips and the resonator. The link with the number of freedom of the lip-reed will be discussed. [Work supported in part by CNRS and NSF.]

\section{$3: 30$}

2pMU8. Annular time-domain acoustic source for horn acoustics. Joaquim B. Agullo and Ana Barjau (Dept. of Mech. Eng., Diagonal, 647, 08028 Barcelona, Spain, agullo@em.upc.es)

The time-domain description of horns is needed in time-domain simulations of acoustical systems such as wind instruments and is very helpful to understand their transient behavior. Unidimensional horn wave equations have been used as a first approximation. The Webster horn equation, however, is too unrealistic for horns with intense flare. The improved horn equation developed by Keefe et al. [Proceedings of the SMAC-93 (1993), pp. 496-500] may be promising. A discretized approach that assumes the horn is made of cylindrical and conical elements with plane and spherical waves, respectively, has been widely used. More general alternatives based on the Kirchhoff integral are attractive because they can handle 2 and 3-D acoustic fields and lead to a surface discretization instead of volume discretization (as required in FEM). For axisymmetric horns, the use of an annular time-domain unit source, not implemented in commercial codes, dramatically reduces the number of elements. This paper presents the development of such a source and its application to time-domain horn acoustics. An example is presented for a cathenoidal horn and its results are compared to time-domain measurements.

\section{3:45}

2pMU9. Determining resonance frequency changes due to bore irregularities in woodwinds. Cornelis J. Nederveen (Acacialaan 20, 2641 AC Pijnacker, The Netherlands) and Jean-Pierre Dalmont (Université du Maine, 72017 Le Mans Cedex, France)

To determine tuning changes due to deviations from an ideal cylindrical or conical bore constituted by holes and by diameter variations, these deviations are modeled by local changes in compressibility and inertance. The magnitude of a compressibility change, being proportional to the volume change of a perturbation, is easily determined, but inertance changes pose more problems, since they may be less than proportional to the volume change. Determining these inertance changes is possible in various ways. An approximation (with a systematic error) is obtained by depicting the 3-D flow as stratified and integrating 2-D results obtained from Schwarz-Christoffel transformations, for which various accurate analytical and numerical solutions are available. Exact results can be obtained with finite difference methods: they are simple, have no systematic error, and only demand a large computing power. Results will be presented for correction impedances at hole junctions, hole ends with various flanges, and toroidal bends. A method of experimental verification and some results will be discussed.

\section{4:00}

2pMU10. Modeling in $3 D$ of directional radiation of curved woodwind instruments. Rene E. Causse and Carole Lheureux (IRCAM, 1 place Igor Stravinsky, 75004 Paris, France)

In a previous presentation [Causse et al., J. Acoust. Soc. Am. 100(4), Pt. 2, 2812 (1996)], the directional pattern of some instruments of the woodwind family was modeled and compared. These instruments, oboe, clarinet, and flute, were chosen for their straight tube and for the fact that the simplification that the holes of the lattice are aligned and oriented in the same direction did not introduce big errors. A second class of instruments of this family are instruments with curved tubes, for example the bassoon, the bass clarinet, or the alto saxophone. The original model was modified to take into account the curves but also the real orientation of the holes. The far-field radiated pressure was calculated at a constant radius on a sphere enclosing the instrument. The main difficulty with this model is expressing the directivity of each opening (holes and bell) in the same coordinate system. In the cases where this leads to excessive calculation, some simplifications are proposed. Model results were compared with experimental measurements of actual instruments. Comparisons of the directional radiation between these instruments and also with straight ones will be presented. Implications for the perception of these instruments will be discussed. 


\title{
Session 2pNSa
}

\section{Noise and Architectural Acoustics: Environmental Noise from Combined Sources}

\author{
Brigitte Schulte-Fortkamp, Cochair \\ Carl Von Ossietzky University, Physics-Acoustics, Carl Von Ossietzky Street, D-26111 Oldenburg, Germany \\ Truls Gjestland, Cochair \\ SINTEF Telecom and Informatics, N-7034 Trondheim, Norway
}

Chair's Introduction-12:50

12:55-1:05 Discussion

Invited Papers

\begin{abstract}
1:05
2pNSa1. Reactions to aircraft noise. Truls Gjestland (SINTEF Telecom and Informatics, N-7034 Trondheim, Norway, truls.gjestland@informatics.sintef.no)

The data from three Norwegian surveys on reactions to aircraft noise have been reanalyzed. Data from more than 9000 respondents have been included. The response to a general annoyance question is compared to the response to questions on special effects concerning speech communication, sleep and rest disturbance, ambient noise, behavioral patterns, etc. The established dose-response relationships will form the basis for new Norwegian guidelines for assessing aircraft noise.
\end{abstract}

\section{$1: 30$}

2pNSa2. The assessment of environmental noise-ISO 1996. Ian H. Flindell (Inst. of Sound and Vib. Res., Univ. of Southampton, UK) and Nicole D. Porter (Natl. Phys. Lab., Teddington, UK)

The concept of a grand "general noise annoyance model" which takes different noise sources and different situations into account by appropriate adjustments or correction factors is very attractive. On the other hand, it is well known that different people respond to different components of the overall situation (acoustic features) at different times, and that this selective attention process can be essentially unpredictable. In order to make progress in this field, it is essential that any new methods of assessment of environmental noise must take this inherent flexibility into account. This paper describes an input-process-outcome model which the authors have been working on as the underlying framework for the current revision of ISO 1996 "Description and measurement of environmental noise." In essence, there are three possible outcomes of assessment (no impact, intermediate impact, and unacceptable impact) and three comparison processes (change in noise, new noise compared against existing background noise, and absolute criteria) which can be applied to the various inputs to determine the assessment outcome. The model allows sufficient flexibility to accommodate most existing national noise assessment procedures, reflects current scientific thinking, and provides a sound basis for choosing the best balance between the costs and benefits of noise control action.

\section{$1: 55$}

2pNSa3. Loudness of combined noises derived from singular and concurrent community noises. Birgitta Berglund and Mats E. Nilsson (Inst. of Environ. Medicine, Karolinska Inst. and Dept. of Psych., Stockholm Univ., Stockholm, Sweden)

It seems logical to assume that the total loudness of combined noises should correspond to the arithmetic sum of the loudnesses of the singular noises as perceived within the combined noise. Although postulated by Powell in the mid 1970s, this hypothesis has not yet been empirically tested. In real life situations, a particular community noise is always perceived within a complex of environmental noises, and therefore, both its loudness and the annoyance it evokes will be associated with the noise immission rather than the noise emission from the singular source. Binary combinations of community noises were tested at up to seven levels of loudness and subjects estimated the loudness of combinations as well as the singular sounds with the aid of magnitude estimation. The loudness summation did not follow Powells arithmetic summation hypothesis. The loudness of singular noises differs when heard alone compared to when heard within the noise combination. As a consequence the type of interaction between loudness of community noises in real life situations (heard within) clearly has implications for the development of better models for total loudness as well as for total annoyance. Earlier models hvae relied on data produced from singular noise sources when heard alone. 
2pNSa4. Psychological evaluation of sound environment with mixed sources. Sonoko Kuwano, Jiro Kaku, Tohru Kato, and Seiichiro Namba (Dept. of Environ. Psych., Osaka Univ., 1-16 Machikaneyama, Toyonaka, Osaka, 560 Japan)

In order to make the experimental situations closer to actual situations, experiments have been conducted in field conditions, where various sounds were audible. The results suggested that generally speaking, $L_{\text {Aeq }}$ was a good measure for the evaluation of environmental noises. However, there were some cases where $L_{\text {Aeq }}$ did not show good correspondence with subjective impressions. On the basis of the results of the field experiments, an experiment was conducted in a laboratory situation with precisely controlled stimuli in order to examine the applicability of $L_{\mathrm{Aeq}}$ to sound with mixed sources. Sounds which consisted of aircraft noise, road traffic noise, and train noise were used. Three events of each noise source were included in 15-min stimuli and the duration of road traffic noise was varied in three stimulus conditions. Subjects were asked to judge instantaneous loudness using the method of continuous judgment by category. After instantaneous judgment was over, overall impression of loudness of each sound source as well as the overall loudness of 15-min stimuli were judged. The following analyses was conducted; (1) comparison among three sound sources, (2) comparison among three experimental conditions, and (3) overall evaluation of sound environment with mixed sound sources.

2pNSa5. Reaction to combined sources of noise may depend on the respondents' interpretation of the questions. R. F. Soames Job (Dept. of Psych., Univ. of Sydney, NSW 2006, Australia, soamesj@psychvax.psych.su.oz.au)

Reaction to multiple sources of noise has proven difficult to explain. The unexpected results of assessment of total reaction to noise may not arise from inadequate integration within the subject nor from complexities of the perception of the noise. This abstract suggests that responses to questions regarding reaction to combined sources may reflect the subjects' interpretation of the relevant questions. Requests for reaction to the total noise load may be interpreted as requesting total reaction to the two sources, or average reaction to the two sources, or total or average reaction to the total noise environment. The consequences of such interpretations by the subjects are not easily predicted. Predictions would be influenced by: whether reaction is a unipolar negative scale versus a bipolar scale from negative to positive; whether the combined reaction is an average or a total; and whether the average or total should include consideration of the times for which each noise is not present. Finally, it should be noted that different subjects may interpret the same question in different ways in relation to these alternatives. Examples of the role of these variables are identified, and a set of models of reaction based on the various alternatives proposed.

2pNSa6. Combined qualitative and quantitative measurements to evaluate noises from combined sources. Brigitte SchulteFortkamp (Dept. of Phys., Oldenburg Univ., D-26111 Oldenburg, Germany, brigitte@aku.physik.uni-oldenburg.de)

Previous studies on assessing combined noises have shown the rating of environmental noises from combined sources as a very complex and difficult task. Disturbance and annoyance caused by the influence of noises require in their complexity an adequate procedure of evaluation. There are different theories to explain those rating results of an overall noise situation which is almost rated lower than the most annoying single source. If sounds are synergistic, the criteria and kinds of sources have to be found which are dominant for the person who assesses the noises. In traditional laboratory studies the context is faded out by the evaluation process, while the context effects in field studies play a decisive role and the evaluation of annoyance caused by noise allows the consideration of different factors in a situation where noises are combined. In a current socio- and psychoacoustical field study the process of assessing the noises and the noises as well are precisely described in interviews and acoustical diaries. In another combined procedure of qualitative-explorative and objective-standardized measurements in a laboratory setting specific noise components causing annoyance and disturbance have been identified. The special methodical procedure and results will be presented.

\section{3:35-3:50 Break}

3:50

2pNSa7. Annoyance accumulation modeling in a community noise annoyance expert system. Dick Botteldooren (Dept. of Information Technol., Acoust. Group, Univ. of Gent, St. Pietersnieuwstraat 41, B9000 Gent, Belgium)

When the state of the sound environment in a region is monitored by tracing the percentage of people who are annoyed by noise, simulation models for community noise annoyance are needed. They allow, for example, the prediction of the impact of various noise control measures. Building such simulation tools is not an easy task because information is scarce and relations between quantities involved are uncertain. Therefore, an expert system was built that makes optimal use of the scarce information and combines as many expert opinions as possible. Consensus mechanisms, representation of uncertain data, and model uncertainty are key factors in this 
model. Combination of annoyance caused by different sources is an important part of the system. Models proposed by various scientists are combined and compared to obtain the most likely global annoyance percentage. Both psychophysical and perceptual models can be applied at the same time. The probability approximation that is used for this task will be introduced. The results of this approach, which combines different opinions, will be shown for a number of example situations.

\title{
Contributed Papers
}

4:15

2pNSa8. Characterization of an urban area's acoustical comfort. Jacques Beaumont (ENTPE-DCGB/URA CNRS 1652, rue M. Audin, F 69518 Vaulx-en-Velin Cedex, France, jacques.beaumont@entpe.fr), Aline Barlet, Christophe Louwrse, and Catherine Semidor (ERIAC, Domaine de Raba, F 33405 Talence Cedex, France)

In this paper a global method to characterize acoustic comfort in urban areas is presented. This method takes into account the relationships between urban forms (shapes, sizes, and material surfaces), the opinions of town-users, and noise descriptors. These descriptors are deduced from a method based on time automatic detection threshold to classify four types of noise. This system allows the study of sound situation in a selfcontained way over a long period (several hours). The influence of building design is estimated according to the visualization of the evolution of these descriptors in 2D and 3D maps all over the studied area. The first results show that noise levels fit well with the architectural characteristics of the buildings (facade irregularities, materials,...). To validate this method, experiments were conducted on a Bordeaux central district. The effects of an urban area's morphology on listeners' noise perception are analyzed from investigations carried out with people living or working in the tested site. The aim of this work is to develop a decision-assisting tool in the acoustic design of urban areas. Environmental considerations, such as noise, must be included in urban planning not only to avoid disturbance, but to provide better satisfaction in living in the city.
4:30

2pNSa9. Sound perception of the urban environment through an audio-visual approach. Stephanie Viollon, Catherine Lavandier (Universite de Cergy, IUT Dept. Genie civil, rue d'Eragny, Neuville sur Oise, 95031 Cergy Pontoise Cedex, France, viollon@u-cergy.fr), and Carolyn Drake (Laboratoire de Psychologie Experimentale, 75006 Paris, France)

Sound and visual environments of cities are usually studied separately without taking into consideration possible interactions between the two sensory modalities. The present research aims at developing an audiovisual approach for assessing the sound perception of the urban environment. It means an innovative approach which takes into account the influence of visual information on the sound judgment. In a preliminary in situ questionnaire, participants rated real-life urban environments along pairs of descriptive adjectives all related to the auditory modality. A Principle Components Factor Analysis revealed four perceptive factors characterizing the perception of the urban environment and also revealed two auditory variables which were the most influenced by the visual setting. Then, with a simulation experimental setting involving the creation of audio and visual artificial fields, a series of semantic differential tests was carried out. Listeners were required to rate the two auditory variables: appeasingstressing and pleasant-unpleasant for eight sound urban environments and for four different visual settings. The results point out that judgments about the two sound variables are significantly influenced by the visual setting. Moreover, improvements in the experimental procedure suitable for testing audio-visual interactions in an urban context are discussed.

\section{TUESDAY AFTERNOON, 23 JUNE 1998}

CASCADE BALLROOM I, SECTION C (W), 1:00 TO 3:15 P.M.

\section{Session 2pNSb}

\section{Noise: Damage Risk Criteria for Impulse Noise}

\author{
James H. Patterson, Jr., Cochair \\ U.S. Army Aeromedical Research Laboratory, P.O. Box 620577, Fort Rucker, Alabama 36362-0577 \\ Guido F. Smoorenburg, Cochair \\ Vasseveldlaan 13A, Soest 3768GK, Holland
}

Chair's Introduction-1:00

Invited Papers

1:05

2pNSb1. The effects of exposure of intense free-field impulse noise on humans wearing hearing protection: Implications for new criteria. James H. Patterson, Jr. (U.S. Army Aeromedical Res. Lab., P.O. Box 620577, Fort Rucker, AL 36362-0577, jhpinal@snowhill.com) and Daniel L. Johnson (EG\&G, MSI, P.O. Box 9100, Albuquerque, NM 87119-9100)

In a series of studies, human volunteers wearing earmuffs were exposed to high-intensity free-field impulse noise which simulated various military weapons noise. By detonating increasing amounts of explosive material, the exposure level was varied from one at which no effects were expected to a maximum level just below the threshold of injury to the lung and upper airway. The number of blasts was varied from 6 to 100. Temporary threshold shifts (TTS) measured 2 to $6 \mathrm{~min}$ after each exposure were used as the measure of effects on hearing. The highest level for each number of blasts at which $95 \%$ of the exposed population would not show a 
significant TTS was used to establish the maximum safe exposure levels as measured in the open and under the earmuffs. These maximum safe exposure levels exceed the exposure limits used in the United States and other countries. Also, these results suggest that the number-intensity trading rule is more shallow than that used in most criteria. The exposures measured under the earmuff provide a good indication of the hazard. Implications of these results for impulse noise exposure criteria will be discussed.

\section{1:30}

2pNSb2. Hearing protectors and hazard from impulse noise: Melding method and models. G. Richard Price and Joel T. Kalb (US Army Res. Lab., APG, MD 21005-5425, dprice@arl.mil)

Studies agree that the protected ear tolerates surprising amounts of energy at very high levels; but with no theory to explain these results, practical application of such findings is like traversing an acoustic minefield. Our modeling of auditory hazard [G. R. Price and J. T. Kalb, J. Acoust. Soc. Am. 100, 2674 (1996)] suggests the physical basis for the ear's resilience is a function of interaction between the hearing protector's effect on the waveform arriving at the ear and the ear's own complex responses to intense stimulation. We believe that the primary physical basis for hazard is mechanical stress at the level of the hair cell; hence assessment needs to take place in the time domain. Calculation begins either with the pressure history measured under a hearing protector or one derived from a free-field pressure and attenuation measures on the protector. The ear model includes an active middle ear muscle system, an amplitude-limited stapes displacement, and an algorithm for calculating hazard in the cochlea which integrates peak upward stresses at the level of the basilar membrane. In the end, the protected ear's apparent resilience is predictable and can be understood as part of a general theory of auditory hazard.

\section{1:55}

2pNSb3. Results of human studies with linear and nonlinear earplugs: Implications for exposure limits. Armand L. Dancer and Pascal J-F. Hamery (French-German Res. Inst. of Saint-Louis, 5 rue du General Cassagnou, BP 34, 68301 Saint-Louis Cedex, France, dancer@nucleus.fr)

Soldiers equipped with linear and nonlinear earplugs have been exposed to large impulses (weapon noises) and audiometric tests have been performed just before and after the exposures. In particular, new nonlinear perforated earplugs (allowing speech communication, detection and localization of acoustic sources) which have been developed at the French-German Research Institute of Saint-Louis are efficient for repeated exposures up to 187-dB peak when they are well fitted [Johnson and Patterson (1996) personal communication]. From various TTS studies it is concluded that: (i) the peak pressure attenuation (measured with a specially designed ATF) grossly underestimates the protection afforded by the earplugs when used in conjunction with the classical criteria for weapon noises (CHABA, MIL-STD, criterion of Pfander, criterion of Smmorenburg). The risk corresponding to an exposure to a slow rise-time impulse (i.e., under an earplug) is much lower than the risk corresponding to an exposure to an impulse of the same peak pressure with an instantaneous rise time, (ii) the $\mathrm{L}_{\mathrm{Aeq} 8}$ attenuation values based on objective insertion loss measurements (ATF) give in most exposure conditions a good evaluation of the auditory hazard, but in some other cases they still underestimate the efficiency of the hearing protectors.

\section{2:20-2:25 Chair's Summary}

\section{2:25-2:35 Break}

\section{Contributed Papers}

\section{2:35}

2pNSb4. Modeling the effect of a hearing protector on the waveform of intense impulses. G. Richard Price and Joel T. Kalb (US Army Res. Lab., APG, MD 21005-5425, jkalb@arl.mil)

Implicit in microphone-in-real-ear (MIRE) or real-ear-attenuation-atthreshold (REAT) tests with a hearing protector is the potentiality that such tests would establish its ability to ameliorate hazard. However, because the sound field under the protector is very different from the free field for which traditional DRCs customarily predict hazard, it is not clear how such measures should be used. The theoretically based mathematical model of auditory hazard we proposed by us as the basis for such assessments calculates motions of structures in the ear [Price and Kalb, J. Acoust. Soc. Am. 100, 2674 (1996)]. Thus phase relationships in waveforms matter and the model must accurately couple them to the ear. Where only REAT data and free-field pressure measures are available, single path conduction through the protector is assumed and minimum-phase processing to calculate waveforms under protectors is used. Empirical tests with free-field impulses and an artificial head test fixture indicate that such an approximation is surprisingly good. With such calculated waveforms or
MIRE measures, a Thevinin-equivalent source to drive the ear which allows the full calculation of auditory hazard with the model is created.

\section{$2: 55$}

2pNSb5. A new nonlinear earplug for use in high-level impulse noise environment. Armand L. Dancer and Pascal J-F. Hamery (French-German Res. Inst. of Saint-Louis, 5 rue du Gener. Cassagnou, BP 34, 68301 Saint-Louis Cedex, France, dancer@nucleus.fr)

A nonlinear earplug allows speech communication, detection and localization of acoustic sources, and prevents hearing hazard from high-peak pressure impulses. Improved physical characteristics of a nonlinear earplug are explained: nonlinear attenuation starting with relatively small level (110 dB peak) and improvement of nonlinear attenuation for high levels. Such an earplug is especially designed against weapon noises (up to $190 \mathrm{~dB}$ peak). The study is based on experimental approach. Moreover, a theoretical model has been developed to explain the experimental results and to optimize the acoustic nonlinear filter dimensions and configuration. The nonlinearity of such a filter is due to one (or several) little hole(s), the acoustic impedance of which is essentially a viscous resistive one and depends on the particulate velocity in its center. 


\title{
Session 2pPAa
}

\section{Physical Acoustics: Acoustic Characterization and Manipulation of Material Properties}

\author{
Sameer Madanshetty, Cochair \\ Mechanical Engineering Department, Kansas State University, Rathbone Hall, Room 339, Manhattan, Kansas 66506-5106 \\ D. Keith Wilson, Cochair \\ U. S. Army Research Laboratory, AMSRL-IS-EE, 2800 Powder Mill Road, Adelphi, Maryland 20783-1197
}

Chair's Introduction-2:15

\section{Contributed Papers}

2:20

2pPAa1. Directional microphone array for measurement in an anechoic wind tunnel. Robin J. Alfredson and Alison K. Wilson (Monash Univ., Clayton, Vic, Australia)

An anechoic wind tunnel in which full-scale acoustic measurements may be conducted on automobiles at speeds up to $200 \mathrm{~km} / \mathrm{h}$ has been constructed. The wind tunnel uses an open jet which is collected and returned in the closed-loop wind tunnel. This design allows for microphones to be positioned outside of the air flow typically some 4 to $5 \mathrm{~m}$ from the vehicle. Thus problems associated with extraneous wind noise on the microphones are largely eliminated but this has resulted in the need to develop microphone arrays which have directional characteristics in order to identify noise sources on the vehicle. A wide range of microphone configurations was examined recognizing the various constraints imposed by cost, data collection facilities, frequency range, and the geometry of the wind tunnel and vehicle. The design that produced an optimum solution was based on an arced array of 10 microphones. The output from each was summed having first been weighted. The array could be moved along the vehicle. Data will be presented indicating the characteristics of this and other arrays as well as the cost of construction.

\section{2:35}

2pPAa2. Phenomenological theory of the translational relaxation times in gases. Allan J. Zuckerwar (NASA Langley Res. Ctr., MS 236, Hampton, VA 23681-0001)

The classical equations governing the translational dispersion and absorption of sound in a gas yield a solution of such complexity as to obscure the relaxational character of the associated viscous and thermal transport processes. The approach taken here is to solve the secular equation by the method of Pade' approximation, which even to the relatively low order $\mathrm{R}_{11}$ yields a remarkably close approximation to the exact solution over a wide range of frequency/pressure ratios. As a result, translational relaxation can be ascribed the attributes of a conventional relaxation process such as relaxation times, relaxation strength, collision numbers, additivity relations, etc. To extend the theory to extremely high values of frequency/pressure ratio, a model is proposed to account for the noncontinuum behavior of the transport coefficients (viscosity and thermal conductivity) as the molecular mean free path approaches the acoustical halfwavelength. The theory shows good agreement with measurements in argon over the classical, Burnett, and transition regions as defined by Meyer and Sessler [E. Meyer and G. Sessler, Z. Phys. 149, 15-39 (1957)].
2:50

2pPAa3. An automated apparatus for measuring sound speeds in hazardous gases. John J. Hurly (Natl. Inst. of Standards and Technol., Bldg. 221, Rm. A111, Gaithersburg, MD 20899)

Our laboratory has developed an automated apparatus for measuring the sound speed in hazardous gases such as those used in processing semiconductors. The sound speed is deduced from measurements of the acoustic resonance frequencies of a gas-filled, cylindrical cavity. After the cavity is calibrated with argon, the uncertainty in the sound speed is less than $0.01 \%$. The $u(P, T)$ data span the temperature and pressure ranges 200-475 K and 100-1500 kPa. From the measured sound speeds we derive the ideal gas heat capacities from the zero-pressure intercepts with a typical uncertainty of only $0.1 \%$. The virial equation of state is also deduced from $u(P, T)$ providing the temperature-dependent density virial coefficients. In comparisons with Burnett measurements, we find that the densities calculated with the derived virial equation of state have uncertainties of less than $0.1 \%$ in density. Some recent measurements in SF6, CF6, and C2F6 will be used to illustrate the systems capabilities. Work in progress on $\mathrm{BCl} 3$ will also be discussed.

3:05

2pPAa4. Ultrasonic flowmeter for the accurate metering of gases for semiconductor processing. Adam M. Calabrese (Natl. Inst. of Standards and Technol., Bldg. 220, Rm. A55, Gaithersburg, MD 20899, adam.calabrese@nist.gov) and Stuart A. Tison (Natl. Inst. of Standards and Technol., Gaithersburg, MD 20899)

Semiconductor production processes such as chemical vapor deposition rely upon accurate control of the flow rate (in the range of 1-1000 standard $\mathrm{cm}^{3} / \mathrm{min}$ ) of several ultrapure, yet highly toxic and/or reactive gases. Currently, thermal mass flow meters (TMFMs) are widely used in industry for these processes. Complications with the use of TMFMs arise because the flow must be divided (due to limited dynamic ranges) and calibration is normally performed with inert gases. In the present research, an ultrasonic Doppler shift flowmeter has been developed to measure gas flows. Measurements of the flow-induced phase shift of an acoustic plane wave propagating in an unobstructed tube, combined with the speed of sound of the gas, are used to determine the mean gas velocity. The combination of the mean gas velocity with measurements of the pressure and temperature allow calculation of the mass flow rate. The experimental system uses stainless steel tubes of diameter $0.05-0.1 \mathrm{~cm}$, and frequencies near $100 \mathrm{kHz}$. No division of the flow is necessary. Prototype meters indicate that sensitivities on the order of $0.75^{\circ} /$ standard $\mathrm{cm}^{3} / \mathrm{min}$ are possible. Experimental data of two prototype systems using different geometries and receivers will be presented. [Work supported by NIST.] 
restriction on the length of object that can be measured using the standard reflectometer. This restriction arises because after the object reflections

2pPAa5. Ultrasonic methods for the on-line real-time characterization of mixing in multiphase systems. Leonard J. Bond and Hans Matthiesen (Univ. of Denver Res. Inst. and Dept. of Eng., 2050 E. Iliff Ave., Denver, CO 80208)

There is currently an increasing interest in developing ultrasonics to provide data that can be used for both process monitoring and control. These developments are being driven by the need to better understand the fluid dynamics and multiphase media interaction phenomena encountered. This is needed to give insights into the process and quantitative data for use in models. This paper will report results obtained through the analysis of dynamic B-scan and digital ultrasonic A-scan data, which are being used for the characterization of mixing in multiphase systems. Results obtained with a model system of sugar mixing into water, under a variety of conditions, will be presented. The insights that are given into the mixing process through the use of a B-scan system will be shown on video. The processes being used to extract data from (I) B-scan images and (II) rf (time-amplitude traces) measured with A-scan systems, to enable the implementation of a mixing index will be reported. Particular attention will focus on the issues of measurement scale and characterization of inhomogeneity. Data will be compared with theoretical model results for ultrasonic properties of homogeneous systems, in particular for suspensions, colloidal systems, and reactions including phase changes.

\section{$3: 35$}

2pPAa6. Water jets and a self-focusing shock pulse source. Bruce J. P. Mortimer (School of Elec. Eng., Cape Technikon, P.O. Box 652, Cape Town 8000, South Africa) and Beric W. Skews (School of Mech. Eng., WITS Univ., South Africa)

The interaction of a liquid pressure pulse with a liquid-air boundary can produce surface deformation and water jet eruption if the incident pressures are high enough. This is important when analyzing damage mechanisms in lithotripsy and in our efforts to produce pulsed water jets [Mortimer and Skews, J. Acoust. Soc. Am. 100, 3548-3553 (1996)]. High-pressure pulses can be generated by either using focusing lens refraction and convergent reflectors, or using self-focusing sources. In this work experimental results using an electromagnetic shock wave source (EMAS) and a new self-focusing source (FEMAS) as discussed. A highspeed digital camera was used to study the water jet production. It was found to consist of two regions; first a high-speed initial jet and second a slower cumulative jet due to the hydrodynamic flow initiated by the source. Results for the FEMAS show a contained cumulative jet as opposed to an unstable jet observed with the EMAS. These results indicate that the device concentrates its on-axis pressure and velocity, a result confirmed by our pressure measurements. However, the operation of the FEMAS was also found to be rather complex in behavior. [Work supported by the Foundation for Research Development.]

\section{3:50-4:05 Break}

\section{4:05}

2pPAa7. Measuring longer tubular objects using acoustic pulse reflectometry. David B. Sharp and D. Murray Campbell (Dept. of Phys., JCMB, King's Bldg., Univ. of Edinburgh, Edinburgh, EH9 3JZ, UK, d.b.sharp@ed.ac.uk)

Acoustic pulse reflectometry is a noninvasive technique for measuring the internal dimensions of tubular objects. A sound pulse is produced using a loudspeaker and injected into the object under investigation via a source tube. The resultant object reflections are recorded by a microphone embedded in the wall of the source tube. Analysis of the reflections yields information about the bore profile of the object. At present, there is a pass the microphone they undergo further reflection at the loudspeaker. These source reflections return to the microphone as unwanted signal. Hence, there is only a finite time over which the object reflections can be accurately recorded which, in turn, limits the length of object that can be measured. In this paper, a method for removing the length restriction is described and preliminary results are presented. The method involves the cancellation of the incoming object reflections at the loudspeaker, thus preventing the formation of source reflections. [Work supported by EPSRC.]

\section{$4: 20$}

2pPAa8. Temperature-scanning ultrasonic measurements in aqueous systems: What is new? Dmitri P. Kharakoz (Inst. of Theoretical and Exp. Biophys., RAS, 142292, Pushchino Moscow reg., Russia)

Ultrasonic measurements are the only high-precision approach to experimental studies of the mechanical part of free energy in liquids, the part directly related to the volume, pressure, and compressibility. During the last decade, a significant progress has been made in the studies of these parameters in aqueous solutions and dispersions of biomolecular materials. The following aspects are considered in this paper: (1) temperature dependence of volume and compressibility as a means to characterize hydration of solutes in water. Important information on the structure and dynamics of bound water can be obtained. (2) Single-frequency simultaneous measurements of sound velocity and absorption and the investigations of fast kinetics. A first-order phase-transition in lipid vesicles (aqueous dispersion) is considered as a process for which unique and very important kinetic information can be obtained by means of such measurements. (3) Sound velocity, compressibility, and volume as a means to characterize conformational states of macromolecules in aqueous solution. Temperature-induced phase transitions in macromolecules (proteins and other polymers). (4) A puzzle of the intrinsic compressibility and nonlinearity of native protein molecules. (5) The acoustic and calorimetric temperature-scanning measurements: A comparative aspect. [Work was supported in part by Russian Foundation for Basic Research, Grant No. 95-04-12226a.]

\section{$4: 35$}

2pPAa9. Acoustic monitoring of the curing process in cement and concrete. Bernhard R. Tittmann, Hasson M. Tavossi (Penn State Univ., Dept. of Eng. Sci. and Mech.), and Frederic Cohen-Tenoudji (Univ. of Paris-7, Denis Diderot, Paris, France)

Acoustic wave velocity and attenuation measurements are used to monitor cement and concrete during hardening. The measured acoustic parameters can then be used to investigate the curing process. The goal of this work is to determine the time required for a given mixture of concrete to be hard enough for the removal of the molds, in order to speed up the construction process. Currently this removal time is unpredictable. In addition concrete, mortar, and cement, before hardening, can be considered as examples of granular materials. The shear, rigidity moduli, and acoustic wave velocity of these materials can differ from the values obtained by applying effective-medium theories and Biots theory. The complete description of the mechanical properties of these materials requires the consideration of discrete nature of the solid constituents and the effects of contacts between the grains. These effects are included in the theoretical description of the process. The methods of acoustic microscopy are also applied to investigate the surface and subsurface physical changes of cement during hardening. Theoretical and experimental results of this investigation will be presented. 
2pPAa10. Model experiments on the evaluation of the cement bond with ultrasonic pulse-echo techniques. Wenxiao Qiao, Guangsheng Du, and Shihong Chi (Dept. of Petroleum Resource, Univ. of Petroleum, East China, Dongying, Shandong 257062, PROC)

Previous research shows that when the transducer is immersed in slurry, it is nearly impossible to determine the cement bond quality of the interface between the cement annulus and formation (Interface II) using the ultrasonic pulse-echo technique. Because strong reflection occurs at the interface between the slurry and casing, most of the portion of the pulse energy is reflected back. In this paper, methods of cement bond evaluation are studied in four different model experiments, in which the transducer is contacted closely to the casing. In these experiments, no matter whether the ultrasonic pulses are normally or obliquely incident to the casing, obvious signals corresponding to the Interface II are observed. Considering the practical implementation of the technique, a thin steel plate is inserted between the transducer and casing. The reflection signal can still be achieved. The model experiments indicate that the transducercontact-casing system can eliminate the influence of the slurry, improve the ratio of signal-to-noise, evaluate the interface between the casing and cement (Interface I), and determine the cement bond quality of the Interface II if the Interface I is well bonded.

\section{5:05}

2pPAa11. Acoustic measurements of condensation/evaporation and crystal growth. Andi G. Petculescu and Larry A. Wilen (Dept. of Phys. and Astron., Ohio Univ., Athens, OH 45701, wilen@ @elios.phy.ohiou.edu)

Using a volume-modulation technique developed to study thermoacoustics, the complex compressibility of a liquid in coexistence with its vapor is measured. Such a system exhibits a distinctive behavior. At low frequencies, the inverse complex compressibility vanishes because the pressure remains at the equilibrium vapor pressure as the total volume is changed slowly. The volume change is accompanied by an exchange of molecules between the two phases. At higher frequencies, however, this exchange is too slow, and the pressure response approaches that which would be expected in the absence of the liquid phase. The presence of the liquid acts effectively as a high-pass filter whose time constant depends on the rate of condensation/evaporation. The application of this technique to the measurement of condensation coefficients of liquids will be discussed. The same technique can also be applied to solid/vapor systems where the results provide a measure of the crystal growth rate. A unique feature of this technique is that the crystal growth rate can be monitored continuously as a function of temperature. [Work supported by Ohio University Research and Sponsored Programs.]
2pPAa12. Anomalous excess noise in inhomogeneous elastic and piezoelectrical solids. Alexander M. Dykhne (Federal Sci. Ctr. “'Trinity," Troitsk, Moscow oblast', 142092, Russia)

The description of the macroscopic behavior of a microinhomogeneous material and the determination of its internal structure may be obtained from its response to external action. The parameters related to the second moments of the field are the simplest to determine. This is the work of deformation. Additional information about the material can be deduced from the higher moments of the respective field. These higher moments are most important in the case of a field with a highly nonuniform spatial dependence. For certain types of the heterogeneity geometry, some points of the elastic field concentration can exist where the field strength increases faster than a certain power function of the distance. The fourth moments of the field can diverge, which will lead to abnormally high excessive noise. The measurement of the excessive noise gives information about the structure of the material. A simple method for determining the high distribution moments of a field is based on measurements of the excessive noise arising from the fluctuations in material constants such as elasticity moduli. This is related to, for example, the deformation of a solid under a fixed external load or the eigenfrequencies of elastic vibrations. In the case of piezoelectrical materials, the excess noise may originate from both elastic and electrical external fields. Their measurement gives more information about the internal structure of the material.

\section{$5: 35$}

2pPAa13. Acoustic pulse diagnostics of relaxation media. German A. Maksimov and Vladimir A. Larichev ( Moscow Eng. Phys. Inst., Kashirskoe sh. 31, Moscow, 115409, Russia, maximov@dpt39.mephi.ru)

The investigation of propagation of acoustic fields in different media is an important source of information about nonideal properties of these media. For study of small-amplitude acoustic waves, the most essential of the nonideal properties are the dissipative-dispersive properties, which are characterized by the frequency dependence of the absorption factor and the phase velocity. These properties can be described in terms of relaxation processes, which arise in the field of acoustic waves. Conventional ways to determine the relaxation times spectrum (RTS) parameters are acoustic spectroscopic measurements of temperature-frequency dependence of absorption and phase velocity of sound in the medium. Currently, such problems can be solved by applying the pulse methods of measurements. In the report the possibilities of acoustic pulse diagnostics of relaxation media are discussed. Parameters of media (such as five moments of RTS) are defined which can be determined experimentally by methods of pulse acoustic diagnostics. The criteria for a pulse excitation source and its registration distances are obtained which provide the most efficient pulse acoustic diagnostics. The comparison of developed approach with the first experiments is fulfilled. 


\title{
Session 2pPAb
}

\section{Physical Acoustics: Half-Spaces and Plates}

\author{
Paul E. Barbone, Chair \\ Department of Aerospace and Mechanical Engineering, Boston University, 110 Cummington Street, \\ Boston, Massachusetts 02215
}

Chair's Introduction-2:15

\section{Contributed Papers}

2:20

2pPAb1. Optical detection of leaky-Rayleigh waves at air-solid interfaces. Laszlo Adler, Christophe Mattei (Adler Consultants, Inc., 1275 Kinnear Rd., Columbus, OH 43212), Michel de Billy, and Gerard Quentin (Univ. Paris 6 and 7, CNRS U.R.A. 17, Paris Cedex 05, France)

Due to the difficulties associated with ultrasonic generation and detection in air in the megahertz region, air-coupled nondestructive evaluation has only recently been explored. In this work, a shear transducer is mounted directly over the edge of a solid specimen so that it can generate a bulk mode in the interior of the solid as well as a Rayleigh wave along the surface. A recently developed optical heterodyne interferometer proved to be sensitive to the leaky-Rayleigh wave in air more than a centimeter away from the solid surface. Results of velocity and attenuation measurements of leaky-Rayleigh waves for several solids will be presented. The technique can also be applied to plates to measure leaky-Lamb velocity and attenuation in air. This method can be used for low optical reflectivity solid surfaces: Thus it can be extended for noncontact nondestructive evaluation applications

\section{2:35}

2pPAb2. Theoretical and experimental study of surface waves on solid-fluid interfaces when the fluid sound velocity is larger than the shear wave velocity in the solid. F. Padilla, M. de Billy, and G. Quentin (Groupe de Physique des Solides, Tour 23, Univ. Paris 7, Denis Diderot, 2 place Jussieu, 75251 Paris Cedex 05, France, Unit assoc. au CNRS n 17, padilla@gps.jussieu.fr)

Two surface waves can propagate on a solid-fluid interface: the Rayleigh and the Stoneley waves. The problem is well known when the fluid sound velocity $\mathrm{cF}$ is lower than the velocities in the solid ( $\mathrm{cS}$ and $\mathrm{cL}$ ). The Scholte-Stoneley dispersion equation has been studied when $\mathrm{cF}$ is greater than the shear wave velocity cS. The numerical results obtained for PVCwater and Plexiglas ${ }^{\circledR}$-water show that there might exist two surface waves whose velocities are lower than cS. The two corresponding roots of the dispersion equation tend to the Rayleigh root of the solid-vacuum interface as the density of the fluid tends to zero. The structure of the two waves has been studied using the formalism of the evanescent plane waves. They are theoretically propagating without attenuation along the interface and exponentially decaying along the direction normal to the interface inside both media. These two waves have been experimentally observed. They exponentially decay along the direction normal to the interface inside both media. The experimental arrival times are in very good agreement with the theoretical ones.
2:50

2pPAb3. A modal reduction technique for the finite element formulation of Biot's poroelasticity equations in acoustics applied to multilayered structures. Franck C. Sgard (LASH, DGCB URA CNRS 1652, ENTPE-Rue Maurice Audin, 69518 Vaulx-en-Velin Cedex, France, Franck.Sgard@entpe.fr), Noureddine Atalla, and Raymond Panneton (GAUS, Univ. de Sherbrooke, QC J1K 2R1, Canada)

Lately, the authors have presented a general technique, referred to as selective modal analysis allowing for a reduction of setup time, computer storage, and solution time of large finite element models involving poroelastic structures. This method uses a dual basis associated with the skeleton in vacuo and the fluid phase in the rigid skeleton limit, respectively. A compact system in terms of modal unknowns is obtained by projecting the coupled system over the dual basis. This technique has been previously tested in the case of the determination of surface impedances and quadratic velocities of single porous materials excited both mechanically and acoustically. It is proposed in this talk to investigate the efficiency of the selective modal analysis technique in the case of multilayered structures made up from a combination of elastic, poroelastic, and acoustic media under various boundary and loading conditions. The theory behind the technique will be recalled together with numerical examples illustrating its performance.

\section{3:05}

2pPAb4. Sound propagation in gas-filled rigid-framed porous media: General theory and new experimental and numerical data. Denis Lafarge, Michel Henry (LAUM, UMR CNRS 6613, 72085 Le Mans, France), Mouaouia Firdaouss, and Jean-Luc Guermond (LIMSI, UPR CNRS 3251, BP 133, 91403 Orsay, France)

The acoustic properties of gas-filled porous media have been the subject of extensive theoretical and experimental work. However, even in the simplest case of rigid-framed materials, most of the theoretical developments found in the acoustical literature remain largely empiric or semiphenomenologic. This is due to the complicated microstructure of porous materials in general. Starting with first principles and using a simple twoscale analysis, a general formulation of the problem of (linear) longwavelength sound propagation in gas-filled media is provided in terms of two independent permeabilities. One of these-the viscous dynamic permeability-is well known. Its thermal analog, the thermal dynamic permeability, is new in the acoustical context. It can be related to a notion of "mean survival time" in diffusion-controlled reactions. The exact connection between the microgeometry and the frequency-dependent dynamic permeabilities is obtained in terms of independent geometrical distribution functions. Useful analogies with the dispersion of electric and magnetic permeabilities are noted. New experimental and numerical data demonstrate the usefulness of the scaling functions proposed by Wilson et al. and Pride et al. Different limitations of the general modeling and the mentioned scaling functions are also noted. 
2pPAb5. Nonlinear propagation of laser-generated acoustical pulses in granulated media. Konstantin Naugolnykh (CIRES, Univ. of Colorado/Environ. Technol. Lab., Boulder, CO 80303), Igor Esipov, Konstantin Matveev (N. Andreev Acoust. Inst., Moscow 117 036, Russia), and Viktor Zosimov (Appl. Acoust. Inst., Dubna 141 980, Russia)

Laser generation of high-intensity broad frequency band acoustical pulses in different fluids and their propagation in water-saturated cobaltmanganese crust (CMC) samples are investigated. Acoustic pulses of amplitude up to $3 \mathrm{MPa}$ and duration about $1 \mathrm{~s}$ are generated in water, oil, and benzene by the action of $\mathrm{CO}_{2}$ on a laser pulse. The propagation of the short acoustic pulses through the water-saturated CMC samples is considered. The complexity of the CMC structure leads to specific nonlinear acoustical features of the process-nonlinear distortion of the acoustic pulse and amplitude-dependent attenuation. Abnormal sound attenuation in the high-frequency band is observed with low-energy probing sound pulses. This effect could be connected with the sound energy capture and its localization as oscillating modes of the structure. This effect vanishes with increasing intensity of the probing pulses, apparently due to the changes in crust structure. At the same time, specific nonlinear distortion of the sound pulse takes place-increasing of the acoustic pulse amplitude leads to decreasing of the negative phase of the passed acoustic pulse. [Work supported in part by Russian Fund for Fundamental Research.]

$3: 35$

2pPAb6. High-resolution spectroscopy of the Lamb modes of a plate near normal incidence. G. Durinck, W. Thys (Katholieke Universiteit Leuven Campus Kortrijk, Interdisciplinair Res. Center, 8500 Kortrijk, Belgium), J. L. Izbicky, and P. Rembert (Universite du Havre, 76610 Le Havre, France)

Using a high-resolution digital oscilloscope and advanced datahandling plate modes near normal incidence, other than those identified by Lamb, were studied. This search was led by the complex roots of the dispersion relation for a plate in water. Solutions with a complex wave number component in the propagation direction indeed reveal near normal incidence the existence of plate modes no one has ever observed to our knowledge. In this paper experimental results are presented at the cutoff frequencies of the S1 and S2 modes of a $1.5-\mathrm{mm}$-thick aluminum plate, and at the cutoff frequencies of the A1 and A2 modes of a 1.5-mm-thick glass plate. The extra resonance predicted by the theory is resolved by looking at the time history of the radiating plate. This is done either by displacing the time window far into the free emission regime or by displacing the receiving transducer in the propagation direction.
2pPAb7. Electric-field influence on Lamb and SH wave properties in $\mathrm{LiNBO}_{3}$ plates. B. D. Zaitsev, S. G. Joshi, and I. E. Kuznetsova (Inst. of Radio Eng. and Electron. of RAS, Saratov Branch, Zelyonaya str., 38 410019 Saratov, Russia)

Presently, acoustic devices using an electrically controlled time delay are highly promising. They include acoustic phase shifters, amplitude and phase modulators, and transversal filters with electrically controlled amplitude-frequency characteristics. Lithium niobate is one of the most promising materials with the anisotropic nonlinear electroacoustic interaction studied for bulk (BAW) and surface (SAW) acoustic waves. This paper is devoted to the theoretical investigation of electrically controlled characteristics of acoustic waves in plates of lithium niobate. The main crystallographic cuts and various thicknesses of plate under the action of different orientations of an external electric field have been investigated. It was confirmed that the dependence of velocity on an electric-field intensity has a complex nature as well as for SAW and BAW. Therefore, even and odd portions of analyzed dependencies have been introduced and studied separately. It was found that mentioned portions are functions not only of wave propagation direction and electric-field orientation but of plate thickness. It allows the changing of the nature of velocity dependence on electric-field intensity from a quadratic law to a linear one. Another advantage is the possibility of providing a significant electric-field intensity by using low voltage due to thin thickness of plate.

\section{4:05}

2pPAb8. Effects of sound and vibrations on tensions and deformations of a stratificated plate. Florian-Paul I. Simion (Dept. of Theoret. Mech., Univ. Politehnica of Bucharest, 313 Splaiul Independentei, 77206, Bucharest) and Gabriela-Cristina D. Simion (Univ. Politehnica of Bucharest, 313 Splaiul Independentei, 77206, Bucharest, simion@freddy.cib.pub.ro)

Effects due to sound and vibrations phenomena in bodies are multiple and more complex than those in the case of sound propagation in air. In the present paper deformations and tensions are analyzed in the case of a plate with eight layers and a solicitation in flexion. Our plate is subjected to a uniform pressure. Let us consider a Cartesian frame $\left(\mathrm{O}, \mathbf{x}_{1}, \mathbf{x}_{2}, \mathbf{x}_{3}\right)$ for our multilayer plate from composite material. Axis $\mathbf{x}_{3}$ is normal to the medium surface of the plate. This plate is composed of eight unidirectional layers and the angles between fibers of a layer and axis $\left(\mathrm{O} \mid \mathbf{x}_{1}\right)$ have the following values: $0, \pi / 2,0, \pi / 2, \pi / 2,0, \pi / 2,0$. Matrix of rigidities for a layer is determined in a rectangular base $\left(\mathbf{x}_{1}, \mathbf{x}_{2}, \mathbf{x}_{3}\right)$ in case $\mathbf{x}_{1}$ parallel to the direction of fibers. A formula of medium deformated surface, arrow in the middle of plate and flexion deformation is determined. Particular application efforts in each layer are computed and their graphs are plotted. 


\title{
Session 2pPP
}

\section{Psychological and Physiological Acoustics: Aspects of Hearing Development}

\author{
Lynne A. Werner, Chair \\ Speech and Hearing, University of Washington, 1417 NE 42nd Street, Seattle, Washington 98105-6246
}

Chair's Introduction-1:00

Invited Papers

1:05

2pPP1. Development of active and passive processes in gerbil cochlea. David M. Mills and Edwin W. Rubel (Dept. of Otolaryngol., Head \& Neck Surgery, Univ. of Washington, Seattle, WA 98195)

Distortion product otoacoustic emissions were monitored in neonatal gerbils during furosemide intoxication, to examine the ontogeny of different mechanisms underlying cochlear responses to acoustic stimuli. Results indicate: (1) Above $2 \mathrm{kHz}$, active processes were functional at adult levels at all ages. (2) In the youngest animals (14 days) the maximum frequency seen for the active response was $16 \mathrm{kHz}$, while in adults it was $40 \mathrm{kHz}$. This limitation on the active frequency response is believed to be due to the underlying passive cochlear mechanical response, and is consistent with the known place code shift. (3) The length of the active amplification zone at the extreme base decreased from $1.2 \mathrm{~mm}$ at $15-19$ days to $0.6 \mathrm{~mm}$ at maturity. (4) At midfrequencies, there was an increase in net active gain from 15 to 19 days, then a subsequent decrease to reach a uniform 25 - $\mathrm{dB}$ gain across frequency by maturity. Overall, these results are consistent with an increase in the rate of gain $(\mathrm{dB} / \mathrm{mm})$ from 15 to 19 days as the endocochlear potential increases to mature levels, followed by a decrease in the length of the active amplification zone throughout the cochlea with maturity. [Work supported by NIH DC00395.]

\section{$1: 35$}

2pPP2. Development of human cochlear function. Carolina Abdala (House Ear Inst., 2100 W. 3rd St., Los Angeles, CA 90057)

The time course for functional development of the human cochlea is not clear. The advent of otoacoustic emissions (OAE) methodology has made it possible to noninvasively probe cochlear function in humans. Distortion product OAE isosuppression tuning curves indirectly reflect cochlear frequency selectivity. They are generated by presenting a third suppressor tone simultaneously with the stimulating tones $\mathrm{f} 1$ and $\mathrm{f} 2$ and increasing the level of the suppressor until a criterion amount of suppression is achieved. When the suppressor level required to achieve criterion reduction in DPOAE amplitude is plotted as a function of frequency, a DPOAE suppression tuning curve (STC) can be generated. DPOAE (2f1-f2) STCs have been generated in adults and neonates to investigate development of cochlear tuning in humans [Abdala et al. (1996); Abdala and Sininger (1996)]. Results of these studies suggest that the human cochlea is resolving frequency in an adultlike fashion by term birth. Other findings also support this contention [Bargones and Burns (1988); Eggermont et al. (1996)]. However, results of a recent study conducted with very premature neonates [Abdala (1997)] suggest that STC width and growth of suppression is not adultlike in premature neonates. These results may be indicative of cochelear immaturity just prior to term birth.

2pPP3. Evidence of prenatal hormonal effects on the auditory system. Dennis McFadden (Dept. of Psych. and Inst. for Neurosci., Mezes Hall 330, Univ. of Texas, Austin, TX 78712, mcfadden@psy.utexas.edu)

There are marked sex differences in a number of behavioral and physiological measures of the auditory system. Evidence is accumulating that some of these sex differences exist at birth and are attributable to the magnitude of prenatal exposure to androgenic hormones, such as testosterone. A generalization emerging from this evidence is that greater prenatal androgen exposures lead to greater masculinization of these auditory responses. At this point, little is known about the modes of action of androgens on developing cochelear structures, but because the degree of masculinization of otoacoustic emissions (OAEs) appears to parallel the degree of masculinization of other brain structures and behaviors, OAEs appear to have the potential to be a valuable, noninvasive window onto the processes of prenatal development and sexual differentiation. Examples of this potential will be discussed. [Work supported by NIDCD.] 
2pPP4. Human auditory system maturation: A neurophysiological comparison between normal-hearing children and children who use a cochlear implant. Curtis W. Ponton, Manuel Don, Betty Kwong, Michael D. Waring (House Ear Inst., 2100 West Third St., Los Angeles, CA 90057), and Jos J. Eggermont (Univ. of Calgary, Calgary, AB T2N 1N4, Canada)

Auditory-evoked responses provide detailed, objective measures of maturational change in the central auditory system from the level of the brainstem to the cortex. Auditory-evoked responses can reflect activity originating from three pathways: the lemniscal and nonlemniscal pathways as well as from a modality nonspecific pathway originating in the reticular activating system (RAS) and its associated thalamic nuclei. Maturational time courses for these pathways were derived from evoked response data recorded from 156 normal-hearing subjects ranging from 5-20 years of age. Analyses of these data indicate that each pathway may have a unique developmental sequence. For normal-hearing children, the lemniscal pathway appears to follow a longer developmental time course than either the nonlemniscal or RAS pathways. For children with implants, the developmental sequences for these pathways are differentially affected depending on onset and duration of deafness. Specifically, maturation of the lemniscal pathway is delayed by an amount that approximates the period of deafness, whereas maturation of the RAS pathway may be related to the age at onset of deafness.

\section{3:15}

2pPP5. Psychoacoustic development in humans and the effects of otitis media on psychoacoustic development. Joseph W. Hall III and John H. Grose (Div. of Otolaryngol./Head \& Neck Surgery, 610 Burnett-Womack CB\#7070, Univ. of North Carolina, Chapel Hill, NC 27599-7070, jwh@med.unc.edu)

Psychoacoustic measures of auditory development in children with normal hearing and fluctuating conductive hearing losses reveal several general trends. First, processing efficiency is usually reduced in young children: they require higher SNRs and are less sensitive to AM. However, underlying mechanisms may be mature: derived measures of frequency selectivity, TMTF time constants, across-frequency processing (CMR and the processing of multiple modulations), and binaural processing (MLD for broadband maskers and sensitivity to off-midline stimuli) are adultlike by age 5 . Nevertheless, key differences remain: across-frequency processing in children is less tolerant of asynchronies than in adults and, for narrow-band maskers, young children have poor binaural masking release, a result which is not due simply to perceptual similarities between signal and masker. A second general trend is that deficits in function due to early conductive hearing loss are not permanent, although the duration of abnormal performance appears to be strongly associated with the specific function being measured. Basic measures of CMR recover by approximately six months but the processing of multiple modulations takes longer. The MLD may take several years to recover. Interpretations of these findings in terms of CNS strategies for analyzing peripheral information will be presented. [Work supported by NIH NIDCD.]

2pPP6. Excessive auditory backward masking and its potential remediation in children with specific language impairment. Beverly A. Wright (Audiol. and Hearing Sci. Prog., 2299 N. Campus Dr., Northwestern Univ., Evanston, IL 60208-3550, b-wright@nwu.edu)

Approximately 5\% of children who are otherwise unimpaired have marked difficulties perceiving and producing spoken language. This disorder is typically labeled specific language impairment. Recent psychoacoustic tests indicate that children with specific language impairment have excessive auditory backward masking, as demonstrated by their extreme difficulties detecting brief tonal signals followed by longer noise maskers. These results are consistent with the hypothesis that specific language impairment results from an auditory perceptual deficit that makes difficult the discrimination of brief, rapidly presented sounds, including phonemes in speech. Subsequent studies using adults with normal hearing and language skills indicate that the interference from a backward masker can be reduced with practice. This learning generalizes from trained to untrained backward-masking conditions and appears to result from improvements in temporal rather than spectral processing. Taken together, these data imply that training in auditory backwardmasking conditions may be a useful intervention for children with specific language impairment. [Work supported by the McDonnellPew Program in Cognitive Neuroscience, NIDCD, the Charles A. Dana Foundation, and March of Dimes.]

\section{4:15}

2pPP7. Frontiers and backwaters of research on the biological bases of hearing development. Edwin W. Rubel (Bloedel Hearing Res. Ctr., Box 357923, Univ. of Washington, Seattle, WA 98195, rubel@u.washington.edu)

The goal of this talk will be to summarize recent advances and current challenges in research on the biological bases of hearing development. Three areas will be focused on: functional and structural changes in the cochlea during the early stages of hearing development; the biological bases of critical periods for inner ear and brain development; and the influence of experience on structure and function of central auditory pathways. In each area the dramatic advances in the amount and quality of descriptive information that has emerged over the past decade will be considered. These data provide a firm foundation on which to test hypotheses regarding the mechanisms that govern development. The contributions that the tools of modern cellular and molecular biology can bring toward providing new answers to questions of developmental mechanisms will be discussed. 


\title{
Session 2pSA
}

\section{Structural Acoustics and Vibration: Memorial Session for Manfred Heckl}

\author{
Kenneth A. Cunefare, Chair \\ G. W. Woodruff School of Mechanical Engineering, Georgia Institute of Technology, Atlanta, Georgia 30332-0405
}

Chair's Introduction-2:15

Invited Papers

2:20

2pSA1. Active control of curve squeal caused by trains. Maria A. Heckl (Dept. of Mathematics, Keele Univ., Staffordshire ST5 $5 \mathrm{BG}, \mathrm{UK})$

Squeal noise is generated by a feedback mechanism. It occurs if two bodies are in contact and move relative to each other so that dry friction acts in the contact area. This can lead to an unstable oscillation driven by the feedback between the friction force and the relative velocity of the bodies. Sound is radiated from one or both oscillating bodies. Some well-known types of noise are generated by this phenomenon, for example squeal and the sound of a bowed string. Curve squeal (the noise produced by the wheels of a train that travels fast around a tight bend) is considered in this paper. The frequency spectrum of curve squeal is dominated by one or a few sharp peaks which correspond to certain bending resonances of the free wheel. Squeal noise can be controlled and even suppressed by a secondary feedback system, consisting of a sensor (to pick up the wheel oscillation or the squeal noise), filter, phase-shifter, amplifier, and transducer (to apply the control signal). This type of control amounts to the stabilization of an originally unstable wheel oscillation and is a special form of active control. A mathematical model for this secondary feedback method will be presented. Numerical results for its effectiveness will be shown.

\section{2:35}

2pSA2. Realization of the Cremer admittance of silencer linings with passive absorber elements. Friedel P. Mechel (Landhausstr. 12, D-71120 Grafenau, Germany)

L. Cremer has shown that the maximum attenuation in a lined duct is obtained when the solution of the characteristic equation lies in the first branch point of that equation. This condition specifies a surface admittance of the lining, the Cremer admittance. Much effort was undertaken in the Institute of Professor Cremer, later directed by Manfred Heckl, to realize this admittance. It will be shown how a computer program can find the material and dimension parameters of a lining with Cremer admittance, after a suitable principal construction was conceived (e.g., multilayer porous absorber, or Helmholtz resonators, etc.). The potentials and the principal limitations of a lining with a Cremer admittance will become visible.

2:50

2pSA3. The influence of barrier edge treatments. Michael Möser (Inst. für Technische Akustik, Einsteinufer 25, 10587 Berlin, Germany)

The paper deals with the reduction of the sound field in the shadow region behind barriers by means of an attached body at the edge of the screen. The reflecting attributes of the barriers hatpiece are described by a locally reacting impedance. The considerations start with a repetition on the diffraction at ideal soft and hard bodies to demonstrate the governing principle of tangential power transport parallel to their surface: The impedance must be chosen in such a way that the tangential intensity near the edge is lowered, such turning the incoming power to "undangerous" directions. Subsequently, the differences due to finite impedances are discussed. The physical principles are demonstrated in a computer animation of the sound fields for the different cases. Theoretical computations give considerable improvement of the level reduction in the shadow zone for not too small angles of diffraction. They are compared with measurement results and practical applications are discussed.

3:05

2pSA4. Induced damping in Heckl's beam experiment. D. Feit and M. Strasberg (Code 7052 and 702, respectively, David Taylor Model Basin, NSWC, 9500 MacArthur Blvd., West Bethesda, MD 20817-5700)

In 1961, Heckl described an experiment showing that the loss factor of a beam in flexural vibration was increased considerably by the attachment of a large number of small cantilevered strips along the span of the beam, their lengths varying so that their frequencies of antiresonance extended over the first five modal resonance frequencies of the beam [J. Acoust. Soc. Am. 33, 640-651 (1961)]. The increase in loss factor occurred even though the total strip mass was much smaller than the beam mass. More recently, an approximate theoretical estimate of the loss factor of Heckl's beam with attached strips, using a simple expression for induced dissipation based on "fuzzy" substructure concepts was presented [J. Acoust. Soc. Am. 99, 335-344 (1996)]. This estimate agreed well with Heckl's measurements at some frequencies, but disagreed at others for unknown reasons. In an attempt to understand the cause of the partial disagreement, new calculations have been performed using a finite-element model of the beam and strips, the loss factor being estimated from resonance bandwidths in steady-state frequency response and transient decay rate. 
For a series of experimental measurements, Manfred Heckl attached strips of damping tape extending outward from the plate edges to increase the plate's damping. He understood that vibrations induced in the tapes would extract vibrational energy from the plates, but did not explore their effects in detail. The present paper presents an analysis of the damping effects that attached wave-bearing systems can produce and illustrates realizations of waveguide absorbers in the shapes of tapered rods and beams. It also presents related experimental data and describes the general potential of waveguide absorbers.

\section{3:35-3:50 Break}

3:50

2pSA6. Heckls influence on SEA development. Richard H. Lyon (RH Lyon Corp, 691 Concord Ave., Cambridge, MA 02138)

Manfred Heckl was at Bolt Beranek and Newman Inc. in the early 1960s during the development of the basic SEA ideas and applications. As various concepts, formulas, and experimental results emerged, he constantly showed how those results related to the earlier work of Westphal, Cremer, and others. Four areas are particularly fresh in my mind - the use of transmissibility notions for connected plates, the use of reciprocity to calculate SEA results, the modal density of cylindrical structures, and the edge damping of plate structures. In every case, Manfred was able to bring fresh insights to the discussions. Although his name is not on many SEA papers, his contributions and influence were strong and are not forgotten.

4:05

2pSA7. The estimation and control of rolling noise from trains. Paul Remington (BBN Technologies, 10 Moulton St., Cambridge, MA 02138)

Throughout his career Manfred Heckl contributed significantly to the body of knowledge that helped to clarify the mechanisms governing the generation of rolling noise from trains. During a brief period at the Technical University in Berlin this author had the opportunity to collaborate with Prof. Heckl and to learn from his insights and expertise in this field. Here an important mechanism for the generation of noise from trains, the interaction force in the zone of contact between wheel and rail produced by the small scale roughness on their running surfaces, will be examined. Analytical techniques for predicting the interaction forces that include the effects of load and wheel and rail profile will be described. The implications for controlling the noise at the source will be examined and the noise reduction achieveable will be estimated using measured wheel and rail roughness data. [Work was supported by the European Rail Research Institute, Utrecht, The Netherlands.]

2pSA8. The multipole method: Contributions of Manfred Heckl and new developments for high-frequency acoustic scattering. Martin Ochmann (Technische Fachhochschule Berlin, Fachbereich Mathematik und Physik, Luxemburger Strasse 10, D-13353 Berlin, Germany)

In 1988, Cremer and Wang suggested spherical field synthesis (one-point multipole method) for calculating sound radiation from nonspherical bodies. Then, Heckl derived a close connection between Cremer's method and the Helmholtz integral equation. Also, he recommended the use of several multipole locations for applying the method to nonconvex radiators (multipoint multipole method). From these ideas, the multipole method has since been extended in several directions. Recent research results were obtained by investigating the high-frequency scattering from a nonconvex structure. This structure consists of a sphere where the positive octant is cut out. The region of the missing octant is called a "cat's-eye," since it acts like a three-dimensional reflector. Hence, depending on the angles of incidence, multiple reflections can occur. The surface may be rigid or characterized by a local absorbing surface impedance. A boundary element model of this structure consists of nearly 8000 elements for accurate consideration of large values of $\mathrm{ka}$. The results of the multipole method are compared with a newly developed iterative solver of the Helmholtz integral equation for high-frequency scattering. Both methods agree well. However, it is shown that commonly used approximations at high frequencies fail, e.g., the plane wave approximation, if multiple scattering occurs.

$4: 35$

2pSA9. Acoustics teaching and Manfred Heckl. Thomas D. Rossing (Phys. Dept., Northern Illinois Univ., DeKalb, IL 60115, rossing@physics.niu.edu)

If allowed to have only a dozen acoustics books in my library, one of them would certainly be Structure-Borne Sound by Cremer, Heckl, and Ungar. This book, which is the epitome of clarity, could only be written by skilled acousticians who are also great teachers. Although the entire book is the result of a close collaboration between these master teachers, the Foreword tells us that the chapters on Damping, Impedance, and Radiation were written by Manfred Heckl. He also animated several of the figures from the chapter on Radiation for a classroom video he created. His lively lectures, often punctuated with thoughtful demonstrations of acoustical phenomena, serve as examples to teachers of acoustics around the world. 
2pSA10. The use of tuned absorbers in minimizing sound power from vibrating structures. Gary H. Koopman, Eric Constans, and Ashok Belegundu (Ctr. for Acoust. and Vib., Penn State Univ., 157 Hammond Bldg., University Park, PA 16801)

This paper presents a methodology for designing quiet shell structures through the use of multiple tuned resonators. Thin shell structures are targeted for noise reduction because of their ubiquity in industry. They are used to cover and protect noisy devices, such as gearboxes and electrical transformers, and are also used to keep dirt and other contaminants away from moving parts, as in the case of automotive valve covers. The method combines a finite element method vibration prediction code, a boundary element method sound power prediction code, and a combined stochastic/gradient-based optimization algorithm for optimizing the locations and parameters (stiffness, mass, and damping) of the tuned absorbers. A design example has been optimized: a thin shell covering a motor/gearbox/brake combination. It is demonstrated that the addition of a small amount of weight $(130 \mathrm{~g}$, or $1.6 \%$ of the weight of the shell) can produce substantial reductions in sound power ( $13 \mathrm{~dB}$ in the targeted a $\frac{1}{3}$-octave band centered at $\left.125 \mathrm{~Hz}\right)$.

\section{5:05}

2pSA11. Recollections of Professor Manfred Heckl. Gideon Maidanik (Carderock Div., Naval Surface Warfare Ctr., David Taylor Model Basin, Code 7030, 9500 MacArthur Blvd., West Bethesda, MD 20817-5700)

Professor Manfred Heckl was one of the scientific giants in the area of acoustics. He combined theory and experiment so well that both were made simple and transparent. I met Manfred at BBN in 1960. I realized he was one of those individuals that you could approach even when the question was not properly formulated. He helped and taught you how to formulate the question and directed you to the answer. He was an exceptional mentor and generous colleague. When he left BBN to return to Germany to assist in establishing Muller-BBN and to be groomed to succeed Cremer's professorship in Berlin, I lost a major impetus in my early development in acoustics. We tried to maintain close professional contacts, but his personal touch was missed and the efforts were not successful. Periodically, we did have face-to-face professional contacts and these were immensely enjoyable and beneficial, but the day-to-day influence was gone and I was poorer for it. Early in our relationship at BBN we became friends and we remained so to the end. I had many opportunities to test this friendship and it withstood intact the passage of time. It is this friendship that I will cherish forever.

$5: 20$

2pSA12. Concentrated lateral excitation of structures. Bjorn A. T. Petersson (Dept. AAETS, Loughborough Univ., Loughborough, Leicestershire LE11 3TU, England)

Supplementing a recent study, aimed at clarifying theoretical requirements regarding models for prediction and design of transmission characteristics for built-up structures [Petersson and Heckl (1996)], vibration transmission relating to lateral force and moment excitation has been investigated for plates and beams of arbitrary depths. Through the associated mobilities, the power transmission and the cross-coupling between lateral translational and lateral rotational components of excitation and motion are studied and the influence of the volumetric nearfield is examined. Complete descriptions are developed for the two associated point-cross mobility elements. From the theoretical results it can be concluded that reciprocity with respect to the point-cross mobility elements is strictly only valid in the hypothetical case of point excitation. The numerical results demonstrate, however, that reciprocity can be invoked in an approximate sense also for realistic excitation conditions. It is shown that a reducttion of a lateral force excitation, remote from the neutral layer, to a combined force and moment excitation at the neutral layer is not applicable except for very small Helmholtz numbers. It is also concluded that the volumetric nearfield affects all mobility elements. Finally, some manageable estimation procedures for the mobilities will be proposed.

2pSA13. Measurement of multicomponent point mobilities on thick plates and deep beams. Christ A. F. de Jong (TNO Inst. of Appl. Phys., P.O. Box 155, 2600 AD Delft, The Netherlands)

The accuracy of power-based prediction methods for sound and vibration transmission in built-up structures, such as statistical energy analysis, is crucially dependent on the estimation of the input power as well as the power transmitted at junctions. These transmissions are governed by multi-component mobilities. When the excitation area is small compared with the structural thickness, the mobilities are strongly influenced by the volumetric near fields. Recent theoretical studies of concentrated excitation of plates and beams of arbitrary thicknesses [Petersson and Heckl, JSV 196(3) (1996) and Petersson ICA'98] have lead to some manageable estimation procedures for the mobility components. This paper describes a series of dedicated laboratory experiments undertaken to validate the estimation procedures. The mobility components are extracted from measured frequency response functions via an inverse method. The experimental procedure and the difficulties encountered are described. The results are compared with the estimated mobilities. 


\title{
Session 2pSC
}

\section{Speech Communication: Prosody, Production, and Voice (Poster Session)}

\author{
Stefanie Shattuck-Hufnagel, Chair \\ Speech Communication Group, Research Laboratory, Massachusetts Institute of Technology, 77 Massachusetts Avenue, \\ Cambridge, Massachusetts 02139
}

\section{Contributed Papers}

\begin{abstract}
All posters will be on display from 1:00 p.m. to 5:00 p.m. To allow contributors an opportunity to see other posters, contributors of odd-numbered papers will be at their posters from 1:00 p.m. to 3:00 p.m. and contributors of even-numbered papers will be at their posters from 3:00 p.m. to 5:00 p.m. To allow for extended viewing time, posters will remain on display until 1:00 p.m. on Wednesday, 24 June. A cash bar will be set-up near the poster area during the Social Hour on Tuesday, 23 June, from 6:15 p.m. to 7:30 p.m.
\end{abstract}

2pSC1. The domain of phrase-final lengthening in English. Stefanie Shattuck-Hufnagel and Alice Turk (Dept. of Linguist., Adam Ferguson Bldg., Univ. of Edinburgh, Edinburgh EH8 9LL, Scotland, turk@ling.ed.ac.uk)

Intonational phrase final lengthening is well established for the final syllable rhyme in English [Klatt (1976), Wightman (1992), Campbell and Isard (1991)]; there is some evidence for lengthening extending further leftward [Langeveld (1997) for Dutch, Berkowitz (1993) for Hebrew], and for post-boundary lengthening of the initial consonant of a new prosodic constituent [Fougeron and Keating (1997), Fougeron (1997), Gow and Gordon (1995), and A. Cooper (1991)]. It is asked whether boundaryrelated duration lengthening extends further in both directions from a prosodic constituent boundary, using prosodically disambiguated sentence pairs like "Please say "Thomasburg or Terraceville and Watertown will play' ", and "Please say 'Michigan or Madison and Vatican will play,", in which pitch-accent and main-word-stress-related lengthening can be distinguished from boundary-related lengthening. Results will be presented for 40 sentence pairs produced by four speakers (normal and reiterant forms) for individual time segments measured across the full extent of each target phrase. Preliminary results from a single speaker suggest that preboundary lengthening can extend leftward to the primary stress of a polysyllabic word. [Work supported by EPSRC.]

2pSC2. Ambiguity in prominence perception in spoken utterances of American English. Laura C. Dilley and Stefanie Shattuck-Hufnagel (Speech Commun. Group, RLE, 36-511, MIT, 77 Massachusetts Ave., Cambridge, MA 02139)

For most words of English in most spoken contexts, the location of the main stress syllable is clear. However, for words in which (a) the main lexical stress is not obvious from the phoneme string alone (e.g., mainstress-ambiguous words like "digest" and "increase," and certain proper names), and (b) the possible positions for the main stress consist of adjacent full-vowel syllables, an ambiguity in the location of the prominence and, consequently, in the identity of the lexical item (for main-stressambiguous words) is observed when these words occur in contexts with certain $F 0$ configurations extending across the adjacent full-vowel syllables (e.g., a rise followed by a peak, or a peak followed by a fall; $F 0$ peaks frequently dissociate from lexically stressed syllables [Ladd (1996)]). In these cases, the syllable affiliation of the main lexical stress is unclear; either the peak or movement (rise or fall) in the $F 0$ contour can be heard as the prominence. This suggests that listeners interpret F0/syllable alignment in light of their language knowledge and a set of perceptual proclivities [Handel (1989)]. When the language fails to specify enough information or two perceptual proclivities are in conflict, perceptual ambiguity can result.

2pSC3. Intonation of noun phrases in Unangan (Eastern Aleut). Alice Taff (Dept. of Linguist., Box 354340, Univ. of Washington, Seattle, WA 98195-4340) and Jacob Wegelin (Univ. of Washington, Seattle, WA 98195-4322)

This paper reports on the intonation patterns of Unangan. The effect of syntactic structure on intonation was investigated. Data for this study are taken from recordings of 12 fluent speakers each providing around 20 sentences in Unangan elicited by English stimuli. Numerical results of entire pitch tracks generated on the Kay CSL 4300B were subjected to statistical analysis. Exploratory plots of batches of sentences were produced using a smoother. When utterances are grouped by speaker, Unangan words have been shown to have an intonation contour whereby each word attains a maximum in its first half, ending lower than it starts. The combination of highs at each word beginning with the downtrending of sentences creates the overall sentence shape of a cascade, each word a step in the falls [Taff and Wegelin, UCLAWPP (1997)]. Building on that study, this investigation looks at plots of same sentences compared across speakers finding that in the possessor/possessed construction (e.g., cormorant's neck) the second word in the phrase is higher in pitch than the first, contrary to the lowering of each successive word described above. Results indicate that intonation in this language is closely related to its syntax. [Work supported by NSF.]

2pSC4. Vowel devoicing and the loss of lexical accent in Tokyo Japanese. Mafuyu Kitahara (Dept. of Linguist., Indiana Univ., Bloomington, IN 47405)

The fundamental frequency of words with devoiced syllables was measured in order to see how the conflict between devoicing and the pitch accent is resolved in Tokyo Japanese. Previous studies found that the pitch after the devoiced accented syllable is elevated in order to compensate for the loss of the accent. Earlier results [M. Kitahara, J. Acoust. Soc. Am. 101, 3195 (1997)] show that the pitch elevation occurs only when there is no phrasal high tone before the devoiced region. The present study further eliminates the possibility of accent shifting by controlling the moraic structure of target words. They contain one or two devoiceable syllables and a moraic nasal in front of the devoiceable region. Four speakers of Tokyo Japanese pronounced target words embedded in a carrier phrase. If 
the accent shifts away from the devoiced syllable, pitch elevation should not occur because the accent is realized somewhere else. Preliminary analysis of the data shows that the accent on the devoiced syllable is not shifted but simply lost under these conditions because the moraic nasal cannot bear an accent in Tokyo Japanese. This result is discussed in connection with intra- and interspeaker variations of the accentual pattern.

2pSC5. The effect of stress on duration in Aleut. Lorna Rozelle (Dept. of Linguist., Box 354340, Univ. of Washington, Seattle, WA 98195, rozelle@u.washington.edu)

Although duration is often one of the physical correlates of stress, it has been claimed [A. Berinstein, UCLAWPP 47 (1979)] that languages with contrastive vowel length do not use duration as a correlate of stress. This study addresses three questions about the phonetic correlates of stress in Aleut, a language with contrastive vowel length. Is duration a correlate of stress in Aleut? If so, does the change in duration caused by stress neutralize the phonemic contrast between short and long vowels? Finally, does stress assignment precede or follow the optional deletion of the final syllable? Data from four native speakers of Pribilof Aleut were analyzed. Vowel duration was measured from the spectrographs produced by a Kay CSL 4300B speech analysis system. Vowels were categorized as stressed or unstressed according to the Aleut stress rule [A. Taff, MA thesis, University of Washington, 1992] and as long or short according to the words dictionary entry. Statistical analysis shows that duration is a correlate of stress, counter to Berinstein's claim. However, despite the change in duration wrought by stress, contrastive ratios between short and long vowels are maintained. In addition, stress is assigned before final syllable deletion.

2pSC6. Temporal structuring of acoustic segments in speech communication. Rodmonga K. Potapova (Dept. of Appl. and Exp. Linguist., Moscow Linguist. Univ., Ostozenca 38, 199837, Moscow, Russia, mglu@online.ru)

The following problems were studied on the domain of the temporal structuring of speech utterance: the determination of algorithms of synthesis of the rhythmic patterns of the utterance, the problem which is often referred to as preprogramming of the temporal organization of the speech signal being part of a more general problem of synthesis (generation) of prosodic patterns of the utterance; the determination of signals used in the information exchange between linguistic and neuro-physiological levels of speech analysis and synthesis; the rules determining the conversion of transformation of the rhythmic patterns of the utterance into real-time intervals between articulatory positions and movements. The character of a durational correlation which has been revealed in this experiment (with the use of the special correlation coefficient $\rho$ and the test of the $H_{0}$-hypothesis) made it possible to define three types of temporal correlation: microcorrelation (for subsound and sound levels), mesocorrelation (for syllabic level), and macrocorrelation (for the level of phrase rhythmic structures-phonetic words). The integral temporal organization of speech utterance is determined on the one hand by a lower level effectcoarticulation, and on the other hand by a higher level effect-the rhythmic patterns of a phrase.

2pSC7. Influence of functional and acoustic parameters of intonation contours on prosody lateralization. Marc D. Pell (McGill Univ., School of Commun. Sci. and Disord., 1266 Pine Ave. West, Montreal, QC H3G 1A8, Canada, czbm@musica.mcgill.ca)

The perception of prosody by adults with unilateral right- (RHD) and left-hemisphere (LHD) damage and subjects without brain injury was assessed through six tasks that varied functional (i.e., linguistic/emotional) and acoustic attributes of a common set of stimuli. Three tasks measured the ability to detect prominence cues in short utterances (focus perception) and three tasks examined the perception of emotional-prosodic features by the same listeners (emotion perception). Within each condition, an initial task tested recognition of each "type" of prosody when all naturally occurring acoustic features (but no semantic features) signalled the target response (baseline). Two additional tasks assessed how changes in (a) duration and (b) fundamental frequency parameters of the stimuli influenced performance on the baseline task within each condition. Results indicated that, irrespective of the availability of specific acoustic parameters, recognition of prosody by both RHD and LHD patients was highly influenced by functional attributes of the stimuli (i.e., LHD patients were selectively impaired in detecting linguistic prominence whereas both groups were impaired in deriving the emotional tone). Brain-damaged patients also displayed irregularities when individual acoustic properties of the stimuli were manipulated, indicating that both functional and acoustic attributes of prosody may be determinants of prosody lateralization.

2pSC8. Syntactic ambiguities and their resolution in prosody: Observations in Japanese, Korean, Mongolian, and Turkish. Shigeru Sato, Young-Sook Choi, and Kei Yoshimoto (Grad. School of Intl. Cultural Studies, Tohoku Univ., Sendai, 980-77 Japan, satos@intcul.tohoku.ac.jp)

Acoustic measurement was made of utterances of syntactically ambiguous sentences in Tokyo and Sendai Japanese, Seoul and Kyongsang Korean, Mongolian, and Turkish to observe prosodic strategies for disambiguation. Materials were sentences of the types ADV-VP1-NP-VP2 and ADJ-NP1-NP2-VP, where the former is ambiguous in terms of adverbial modification, ADV modifying either VP1 or VP2, and the latter creates ambiguity for the adjective ADJ modifying either NP1 or NP1+NP2. For these constructions, the four languages show identical constituent structures, and accordingly create same ambiguities. After defining the syntactic depth of a boundary, $F 0$ of the phrase before and after the boundary, and duration of the syllable and pause before the boundary were measured. The results for differentiating the syntactic depth: (1) Tokyo Japanese and Kyongsang Korean make use of $F 0$ rise after the boundary, (2) Seoul Korean lengthens the duration, (3) Turkish uses $F 0$ rise before the boundary followed by a pause, and (4) pause is the indication for Sendai Japanese and Mongolian.

2pSC9. The effects of discourse focus and lexical accent on the prosodic phrasing in Japanese. Young-Sook Choi, Shigeru Sato, and Kei Yoshimoto (Grad. School of Intl. Cultural Studies, Tohoku Univ., Sendai, 980-77 Japan, yschoi@insc.tohoku.ac.jp)

Measurement of $F 0$ was made of utterances of Japanese sentences to observe behavior of intonation contours with varied focus assignment and lexical accent specifications. Materials were 80 sentences of rightbranching structures of the type NP1-NP2(-NP3)-VP, where 16 have two three-mora NP's and 64 three NP's, including all the permutations of NP's, each with one of the four lexical accent types. The utterances were generated in question-answer discourse contexts so that in a sentence, one NP was always focused. The results are: (1) focus assignment has no significant effect on NP1, (2) NP2 and NP3 are significantly higher when focused than when not focused, and (3) focused/unfocused NP2 preceded by unaccented NP1 is significantly higher than those preceded by accented NP1, and so is NP3 preceded by NP2 with the same conditions. These suggest that focus assignment on NP2/NP3 requires rephrasing there, that 
a lexical accent of an NP narrows the pitch range of the following phrase, and that the prosodic status of focus assignment is lower than that of the lexical accent.

2pSC10. Effects of prosodic structure on consonant weakening. Lisa M. Lavoie (Dept. of Linguistics, Cornell Univ., Morrill Hall, Ithaca, NY 14853, LML1@ cornell.edu)

While the effects of lexical stress are most obvious on vowels, consonants also show evidence of prosodic structure. In English, stress conditions the flapping of alveolar stops and Turk ["Effects of Position in Syllable and Stress on Consonant Articulation," Ph.D. dissertation, Cornell University, 1993] has shown that labial stops also shorten in the flapping environment. This study investigates the strength of intervocalic consonants as a function of word stress, position-in-word, and speech rate. Two stress pairs of closely matched disyllabic words (one for word-initial, one for word-medial) were recorded for 16 English consonants at three rates of speech. Words for [v] are as follows, with the stressed syllable capitalized: VENdor, veNEER; SEver, seVERE. Results for fricatives will be reported, including the following tendencies for fricatives which are the onset to an unstressed syllable: voiced fricatives are more fully voiced, some fricatives are shorter, more formant structure is evident in the noise, and transitions to the following vowel may be longer. Findings for each speech rate will be compared to determine how the rate may impact consonant behavior. Each correlate will be evaluated with respect the hypothesis that consonants in unstressed syllables are more vowellike.

2pSC11. Intergestural coordination adjacent to multiple prosodic boundaries. Dani Byrd (Haskins Lab., 270 Crown St., New Haven, CT 06511-6695)

Recent work has demonstrated that progressively larger prosodic boundaries result in increasing amounts of lengthening for consonant gestures both preceding and following the boundary. This experiment extends these findings by considering kinematic data collected with a magnetometer to determine if: (1) vowel gestures demonstrate lengthening patterns comparable to those of consonant gestures due to adjacent prosodic boundaries, and (2) the relative timing or intergestural coordination between consonant and vowel gestures is affected by adjacent boundaries. Eighty tokens were recorded for each of three speakers using magnetometry for a / . . amə(\#)mi . . . / sequence with five differing boundary conditions: (1) no boundary, (2) a simple word boundary, (3) a boundary between elements in a list, (4) a vocative name boundary, and (5) an utterance boundary. Durations of the bilabial closings and openings, duration of the vocalic tongue raising movement from [a] to [i], and temporal latencies between the pre- and post-boundary [m]'s and the [i] articulation were determined. All subjects demonstrated lengthening of the vocalic tongue raising for larger boundaries, although they differed in whether the lengthening was more pre- and post-boundary. Furthermore, two subjects had no systematic changes in relative /\#CV/ timing, but one subject showed relatively later vocalic peak velocities for larger boundary conditions. [Work supported by NIH.]

2pSC12. Nontraditional acoustic features of focus. H. Timothy Bunnell, Stephen R. Hoskins, and Debra Yarrington (The duPoint Hospital for Children and Univ. of Delaware, 1600 Rockland Rd., Wilmingon, DE 19803)

Natural productions of the sentence "Bob bought Bogg's box" in which focus was varied over each of the four words of the sentence were altered to produce prosodic cue neutralized versions. The alterations were applied singly and in all possible combinations to form eight experimental versions of each original sentence (the original and seven cue-neutralized versions). The original sentences as spoken by eight talkers and their cue-neutralized versions were presented to listeners with the task of identifying the focused item in the sentence. Results indicated that (a) overall, $F 0$ cues were more important than either amplitude or duration cues in signaling focus, (b) the importance of amplitude and duration cues was greatly enhanced when $F 0$ cues were neutralized, and (c) in many cases, identification of focus remained above chance after all three acoustic features were neutralized. The present study reports analyses of the residual acoustic features which continue to convey focus when amplitude, duration, and $F 0$ are neutralized. [Work supported by NIDRR and the Nemours Research programs.]

2pSC13. Baby word rhythm preferences of Japanese infants. Akiko Hayashi (Faculty of Education, Tokyo Gakugei Univ., 4-1-1, Nukui-Kita, Koganei-Shi, 184 Japan, hayashi@u-gakugei.ac.jp), Kyoko Yoshioka (Tokyo Gakugei Univ., Koganei-Shi, 184 Japan), and Reiko Mazuka (Duke Univ., Durham, NC 27708-0085)

For infants acquiring English, many studies have shown that the dominant stress pattern of the language (viz., strong-weak syllable sequence) plays a critical role in perception, segmentation, and early speech production. Questionnaires to Japanese mothers revealed that many words they use in child-directed speech take the form of $\mathrm{XsX}$ or XsXs ( $\mathrm{X}=$ regular mora; $s=$ special morae). It was hypothesized that this sequence might count as a dominant rhythmic pattern for Japanese infants. Using a headturn listening preference paradigm, 8-10-month-old Japanese infants were found to show a preference in listening to lists of pseudowords that conform to the XsX pattern when compared to another list of words with three regular morae $(\mathrm{XXX})$. Four- to six-month-old infants showed no such preference. An additional experiment eliminated the possibility that the result of the first experiment was simply based on the presence or absence of special morae in the lists. The results will be discussed in relation to the increasing body of English data to examine how infants might learn to take advantage of language-specific rhythmic patterns in language development.

2pSC14. The relationship between spectral properties and perceptual evaluation of hypernasality in children with cleft palate-vowel /i/. Ryuta Kataoka, David Zajac, Robert Mayo, Donald Warren (UNC Craniofacial Ctr., Univ. of North Carolina, Chapel Hill, NC 27599-7450), and Kaoru Okabe (Coretex Corp., Tokyo, 192 Japan)

The purpose of this study was to examine the relationship between spectral characteristics and the perceptual evaluation of hypernasality in speakers with cleft palate. Speech samples from 32 cleft palate patients (average age 9.3 years) and 5 normal subjects (average age 9.4 years) were used in this study. The isolated vowel /i/ produced by each subject was filtered using 16 one-third octave bandpass filters from $200 \mathrm{~Hz}$ to $6.3 \mathrm{kHz}$ to obtain a $1 / 3$ octave spectrum. These spectra were normalized using the total power level of the $161 / 3$ octave bands. Three sophisticated listeners rated the speech samples using a six-point equal-appearing interval scale. Average spectra obtained from the hypernasal and the normal groups were compared. Spectral characteristics of hypernasality in the vowel /i/ were identified as a rise in power level between the first formant $(F 1)$ and the second formant $(F 2)$ and a rise in power level of $F 1$. Multiple regression analysis revealed a high correlation $(r=0.81)$ between the averaged perceptual scores and four levels of $1 / 3$ octave bands: the band containing $F 1$, the first, the second, and the fourth bands from $F 1$. [Work supported by NIDR Grant Nos. DE07105 and DE06957.] 
2pSC15. Effects of levodopa on finger and articulatory movements in Parkinson's disease. Michele Gentil and Pierre Pollak (INSERM, CHU Clinique Neurologique BP 217, Grenoble 38043 Cedex 9, France, michele.gentil@ujf-grenoble.fr)

Efficacy of levodopa therapy on finger and articulatory movements in Parkinson's disease was studied. The production of isometric force was estimated in both conditions, without and with levodopa in 14 patients with a diagnosis of idiopathic Parkinson's disease (the mean duration of symptoms was 9 years). Forces generated by the upper and lower lips, tongue, and right and left forefingers in the presence of visual feedback were measured by means of force transducers. Moreover motor disability of patients was assessed in each condition. Fourteen control subjects also participated in this study. The beneficial effect of levodopa on finger motor disorders of patients was observed as indicated by better motor scores and improved production of finger forces. In contrast, the production of articulatory forces of patients was either not improved or aggravated by levodopa therapy. These results suggest that cerebral nondopaminergic lesions are probably responsible for the impairments of parkinsonian speech.

2pSC16. Stop-consonant production by dysarthric speakers: Use of models to interpret acoustic data. Kelly L. Poort (Res. Lab. of Electron. and Dept. of EECS, MIT, 50 Vassar St., Cambridge, MA 02139)

Acoustic measurements have been made on stop consonants produced by several normal and dysarthric speakers. The acoustic data were previously recorded by H. P. Chang and H. Chen at MIT. In the present study, various aspects of production following release of the oral closure were quantified through the use of acoustic measures such as spectra and durations of noise bursts and aspiration noise, as well as shifts in frequencies of spectral prominences. Through comparison of these measurements from the normal and dysarthric speech, and based upon models of stopconsonant production, inferences were drawn regarding articulator placement, rate of articulator release, tongue-body movements, and vocal-fold state. The dysarthric speakers deviated from normals particularly with respect to alveolar constriction location, rate of release, and tongue-body movement into the following vowel. For example, the lowest front-cavity resonance in the burst spectrum of an alveolar stop is normally in the range $3500-5500 \mathrm{~Hz}$. For three of eight dysarthric speakers, the range was lowered to $1500-2800 \mathrm{~Hz}$, indicating either placement of the tongue tip further back on the palate or formation of the constriction with the tongue body in a location similar to that of a velar stop. [Work supported in part by NIH Grant DC00075.]

2pSC17. Formant trajectory characteristics in persons with Parkinson, cerebellar, and upper motor neuron disease. Gary Weismer and Jill Wildermuth (Dept. of Communicative Disord. and Waisman Ctr., Univ. of Wisconsin-Madison, 1500 Highland Ave., Madison, WI 53705)

Vocalic nuclei appear to have a good deal of importance in understanding the speech motor control deficit in dysarthria. Research in our laboratory over the past ten years has shown that the slope of formant transitions and the size of the acoustic vowel space are both predictive of the speech intelligibility deficit associated with amyotrophic lateral sclerosis (ALS). Moreover, certain aspects of formant trajectories, such as transition duration and transition extent, seem to provide useful inferences about the articulatory disorder in ALS and in Parkinson disease (PD). In the present study the investigation of vocalic nucleus production in persons with dysarthria is broadened, by reporting on formant trajectory characteristics of persons with Parkinson, cerebellar, and upper motor neuron disease due to stroke. The hypothesis of pathology-specific trajectory characteristics is evaluated, and some methodological considerations concerning the parametrization of formant trajectories are discussed. [Work supported by NIDCD DC00319.]
2pSC18. The effects of utterance length on temporal control in aphasia. Shari R. Baum (School of Commun. Sci. and Disord., McGill Univ., 1266 Pine Ave. W., Montral, QC H3G 1A8, Canada, insr@musicb.mcgill.ca)

By comparing the same syllables produced in a variety of utterancelength conditions, the present investigation examined the hypothesis that fluent aphasics exhibit a shorter-than-normal speech planning domain, whereas nonfluent aphasic patients exhibit impaired speech timing at all levels. Subjects included nonfluent aphasics, fluent aphasics, RHD controls, and normal controls. The base stimuli were 10 monosyllabic nouns from which 10 bisyllabic words were derived. Each of the 20 words also appeared medially in the context of a short (4-6 syllable) and a long (8-10 syllable) sentence. Productions were elicited two times each and recorded for acoustic analysis. Durations of the target monosyllable as a proportion of whole utterance durations were computed. Preliminary analyses revealed that normal speakers produced target syllables with shorter durations in 2-syllable relative to 1-syllable words in both long and short utterances. Target syllables were shorter in the long sentences, as expected. A similar pattern was found for the other speaker gruops, but the magnitude of the differences was larger in the fluent and nonfluent aphasic patients productions. Moreover, the nonfluent speakers' proportional durations were aberrant due to abnormally long sentence durations. Results are discussed in rlation to theories of temporal control in brain-damaged patients. [Work supported by MRC.]

2pSC19. Source modeling of severely pathological voices. Bruce R. Gerratt, Jody Kreiman, Norma Antonanzas-Barroso, Brian Gabelman (Div. of Head/Neck Surgery, UCLA School of Medicine, 31-24 Rehab. Ctr., Los Angeles, CA 90095-1794), and Abeer Alwan (UCLA, Los Angeles, CA 90095)

Previous studies using a simplified LF source model [Qi and Bi, J. Acoust. Soc. Am. 96, 1182-1185 (1994)] to describe pathological voice sources indicated that model fit to the return phase is particularly poor. To examine the perceptual importance of differences in how the return phase is modeled, various functions were fitted to the source waveforms that had been obtained by inverse filtering. Voices were then resynthesized using these different source functions, and the resulting signals were compared perceptually to the original natural voice and to versions synthesized with LF modeled sources. The perceptual importance of changes in closing phase modeling for these severely pathological voices will be discussed, as will the range of functions necessary to obtain perceptually satisfactory synthesis of severely deviant voice sources. [Research supported by NIH.]

2pSC20. Speaking rate-induced acoustic variability in Parkinson's disease. Kris Tjaden (Dept. of Commun. Disord., San Diego State Univ., 5500 Campanile Dr., San Diego, CA 92182-1518, Tjaden@mail.sdsu.edu)

The idea that speech consists of overlapping gestures that slide with respect to one another suggests that measurable kinematic, and hence, acoustic differences should characterize utterances produced with varying degrees of gesture overlap. [Saltzman and Munhall, Ecolog. Psych. 1, 333-382 (1989)]. Further research suggests that a simple acoustic model of overlapping gestures [Weismer et al., J. Phon. 23, 149-164 (1995)] accounts for some of the spectral and temporal variability in speech produced by individuals with apraxia of speech. One implication is that articulatory deficits associated with motor speech disorders can be modeled, in part, as disruptions in gesture overlap. The extent to which a model of overlapping gestures accounts for acoustic variability in other motor speech disorders is largely unknown. The present study explored the extent to which a simple acoustic model of overlapping gestures accounted for speaking-rate induced variability in $F 2$ trajectories produced in target words by individuals with Parkinson's disease (PD), young adults, and older adults. $F 2$ onset frequency was used as an acoustic measure of 
overlapping consonantal and vocalic gestures; $F 2$ onset frequency also was used to predict across-repetition temporal variability in $F 2$ trajectories.

2pSC21. A comparative study of speech motor programming in stutterers and nonstutterers. N. Aravind Kumar and S. R. Savithri (Speech and Hearing, All India Inst. of Speech and Hearing, Mysore 570 006, India)

The present study was undertaken to verify the credibility of theories that attribute the locus of stuttering aetiology to be a central speech planning dysfunction. Ten stutterers and ten nonstutterers participated in this study which utilized a choice reaction time paradigm to determine the effects of three independent variables, viz., word length, phonetic complexity, and linguistic complexity on dependent variable-speech reaction time (SRT). By varying the response complexity in this manner it was intended to manipulate the response preparation in a way that if stuttering depends on a premotor programming, then the response complexity would adversely affect response preparation time and this effect of "complexity" would be greater for stutterers (with aberrant speech programming) than for nonstutterers. The results obtained supported the above and significant differences were elicited in both within and across group conditions. However, some peculiar "effects," viz., "paradoxical complexity effect" and "motor ceiling effect" were also evidenced and are subsequently discussed. The results of the study suggest that the stutterers may be endowed with inefficient motor programming capabilities that are aggravated by increased production demands especially under time stress conditions.

2pSC22. Modeling of articulatory dynamics using cascaded firstorder systems. Yorinobu Sonoda and Kohichi Ogata (Faculty of Eng., Kumamoto Univ., 2-39-1 Kurokami, Kumamoto, 860 Japan, ogata@eecs.kumamoto-u.ac.jp)

This paper proposes modeling of articulatory dynamics using cascaded first-order systems. The model, which consists of simple first-order components, can be a good approximation for the behavior of a complex system. In the paper, the approach to modeling the articulator movements was performed on several articulatory organs: tongue, jaw, etc. Parameters describing the model were obtained from observed articulatory data in such a way that time patterns of impulse response of the model were approximated to velocity ones of movements. Good approximations were obtained on time patterns of articulation for vowels and consonants. Therefore, successive movement patterns for continuous speech including consonants were approximated by combining several models with high accuracy. By modeling articulatory dynamics for vowels and consonants based on articulatory measured data, complex articulatory patterns in a form of $[-\mathrm{V} \mathrm{C} \mathrm{V-]} \mathrm{were} \mathrm{synthesized} \mathrm{and} \mathrm{the} \mathrm{time} \mathrm{instant} \mathrm{of} \mathrm{input} \mathrm{com-}$ mands for articulation of vowels and consonants were estimated in a connected speech sound including consonants. [Work supported by SCAT.]

2pSC23. Testing a normalization procedure for articulatory recovery. Richard S. McGowan (Sensimetrics Corp., 26 Landsdowne St., Cambridge, MA 02139)

In order to recover articulatory movement from human speech in the analysis-by-synthesis procedure used in a current project it is necessary to adapt, or normalize, the anatomy of the vocal tract of an articulatory synthesizer. A normalization procedure was tested in which the vocal tract length and the functions determining the conversion of midsagittal distance to cross-sectional area were adjusted until there was an acoustic match between the synthesizer and the human over a selected set of utterances. Testing of the normalization procedure was performed using vowel data on two subjects from the Wisconsin x-ray microbeam data set to find how many utterances with known midsagittal shape and acoustic information were necessary so that the adjusted synthesizer vocal tract was able to predict output of novel utterances. Another part of the normalization procedure is to find a mapping between the settings of the articulators of the synthesizer and the corresponding midsagittal shapes of the human vocal tract. Neural networks were trained to map from the settings of the articulatory synthesizer to the positions of pellets over limited sets of vowels. The ability of these networks to generalize was tested with novel vowels. [Work supported by grant DC-01247 from the NIH.]

2pSC24. A task-dynamic approach to gestural anticipation in speech: A hybrid recurrent network. Elliot Saltzman (Haskins Labs., 270 Crown St., New Haven, CT 06511, saltzman@haskins.yale.edu) and Suvobrata Mitra (Univ. of Connecticut, Storrs, CT 06269)

Recent simulations of the anticipatory timing of speech gestures within the task-dynamic model of gestural patterning are described. To accomplish this, a sequential neural network architecture is used in a simplified dynamical system in which the network's outputs define the activations of gestures within a set of vocal tract constrictions ("tract variables") whose dynamics are second order. These activations act only to insert target values into the tract-variable dynamics; the stiffness and damping coefficients are fixed. The forward dynamics, from current tract-variable state and target inputs to the next tract-variable state, are then modeled by a second recurrent network whose outputs are fed back to the sequential network. In this manner the simulated articulatory timing patterns are sensitive both to the intended linguistic sequence and to the articulatory dynamics themselves. Simulations will be described suggesting that anticipatory gestural timing is defined along a simple implemented continuum between rigid time-locking and unrestricted lookahead behavior. Temporally elastic anticipatory behavior such as that reported for French lip protrusion falls naturally within this continuum. These results provide an elegant account of interspeaker and interlanguage differences in anticipatory gestural timing. [Work supported by NIH.]

2pSC25. An interactive construction system of three-dimensional vocal tract shapes from tomogram. Tohru Yokoyama, Nobuhiro Miki, and Yoshihiko Ogawa (Faculty of Eng., Hokkaido Univ., Sapporo, 060 Japan, yokoyama@kairo3.hudk.hokudai.ac.jp)

A new interactive system is developed to construct the 3-D vocal tract shape from tomogram, for the purpose of understanding the relationship between the speech production process and the geometrical figure of the human vocal tract. The shaded 3-D vocal tract shape can be seen with this system from any direction, by construction from a set of 2-D images measured by, for example, MRI or x-ray CT, and basic geometrical information is obtained for estimating the area function and transfer function, or creating the mesh model for 3-D FEM. For constructing a 3-D vocal tract shape, the following three operations are conducted for the system. First, the 3-D locations of the tomogram images are interactively determined. Second, the contours of the air tract in the images are extracted after several image processings. Third, the wire frame model of the vocal tract, including a branching case, is constructed computationally from the extracted contours. The system is developed using the $\mathrm{C}++$ language as an object-oriented programming language, Motif toolkit as GUI, and PEX as 3-D graphic libraries. The proposed system provides a convenient tool for speech research on the $\mathrm{X}-\mathrm{Window}$ environment.

2pSC26. Palate shape effects on characteristic vowel tongue postures. Mark K. Tiede (ATR Human Information Processing Res. Labs., 2-2 Hikaridai, Seika-cho, Soraku-gun, Kyoto, 619-02 Japan, tiede@hip.atr.co.jp)

In this work MRI vocal tract data obtained during sustained vowel production is used to investigate the interaction between individual speaker differences in hard palate shape and the compensatory adjustments to tongue posture needed to tune language-specific vowel formant resonances. PCA techniques developed in previous work [Tiede and Ye- 
hia, J. Acoust. Soc. Am. 100, 2658(A) (1996)] were used to characterize three-dimensional tongue and palate shapes for vowel inventories of five Japanese and five American English subjects. Parametrized shapes for individual speakers were compared to a normalized mean tract shape pooled across subjects for each language. Preliminary results show that the axis of tongue body displacement is associated with oral cavity depth (OCD, measured from the teeth to the rear pharyngeal wall): Shorter OCD subjects showed greater vertical /a/ versus /i/ differences, and longer OCD subjects used more of a horizontal component. Systematic effects of palate vault shape on tongue constriction location have also been observed. These results are consistent with the speaker-specific articulatory (" $\mathrm{A}$ ", space) adaptations suggested in previous work [Honda et al., Proc. ICSLP96, 784-787 (1996)].

2pSC27. Characteristics of phonation offset and onset in normal adults and children. Laura L. Koenig (Long Island Univ., Brooklyn, $\mathrm{NY})$

A major goal of research into the dynamics of vocal fold vibration is to specify the physical requirements for self-sustained oscillation, i.e., phonation. In recent years, theoretical and modeling studies have sought to define the necessary conditions for initiating phonation in laryngeal models of varying complexity, focusing especially on requisite pressure levels (the phonation threshold pressure), structural configurations (glottal width and the glottal convergence angle), and their interrelations. Conditions surrounding phonation offset have received somewhat less attention but are generally understood to involve different scale values of the same set of variables. This paper investigates voicing behavior around abduction for $/ \mathrm{h} /$ in normal English-speaking adults and 5-year-olds. Recordings consist of intraoral air pressure and oral airflow collected using a Rothenberg mask. DFT analyses of airflow signals from speakers whose /h/Us had voicing interruptions reveal that spectral changes accompanying phonation offset differ from those seen at phonation onset. Speakers show various individual patterns but the difference is typically most evident in the amplitude and bandwidth of the first harmonic. These results add to the empirical literature indicating that initiation and cessation of voicing do not occur under identical conditions. [Work supported by NIH.]

2pSC28. Speaker identification using noncontemporary speech samples. Harry Hollien and Reva Schwartz (Inst. for Adv. Study of the Commun. Processes, 50 Dauer Hall, Univ. of Florida, Gainesville, FL 32611)

There are two noncontemporary elements important to speaker identification. In one, listeners are asked to make identifications at various latencies after hearing the speaker (time of identification is noncontemporary) or, in the second, samples of the speaker's voice are obtained at markedly different times (noncontemporary samples). It has been found that memory for voice decays over time in the first case; hence it has been assumed that the noncontemporary samples pose just as difficult a challenge. AN aural-perceptual speaker identification project was carried out to test this second relationship. Noncontemporary samples, with latencies of 4,8 , and 32 weeks, and then 6 and 20 years, were studied. It was found that mean correct identification was initially reduced by $15 \%$ and this level was sustained for up to 6 years. It was only after 20 years that levels dropped significantly (to 33\%). It is concluded that listener's efficiency in identifying noncontemporary speech will show only modest decay over time and, hence, its use ordinarily should have only a minimal effect on the speaker identification process.

2pSC29. Perception of intoxication effects on speech. Harry Hollien and Gea DeJong (Inst. for Adv. Study of the Commun. Processes, 50 Dauer Hall, Univ. of Florida, Gainesville, FL 32611)

Can speakers, especially actors, effectively mimic the speech of intoxicated individuals and can they volitionally reduce speech degradation resulting from this behavioral state? Auditors were tasked to determine if actors sounded more inebriated when simulating it than when they actually were intoxicated and if they sounded less intoxicated (than they were) when attempting to sound sober. Young, healthy actors, chosen on the basis of a large number of selection criteria were required to produce several types of utterances: (1) during a learning phase, (2) when sober, (3) at three simulated levels of intoxication, (4) during actual, and parallel, levels of controlled intoxication, and (5) at the highest level of intoxication but when attempting to sound sober. Two (counterbalanced, ABX) auralperceptual procedures were carried out with 40 listeners. It was found that these auditors rated the speakers as being more intoxicated when sober, but simulating drunkenness, $88 \%$ more often than when actually intoxicated. They also were judged as sounding less inebriated (than they were), when attempting to sound sober, $61 \%$ of the time. The bases for these relationships will be discussed.

2pSC30. Perceptually motivated modeling of noise in pathological voices. Brian Gabelman, Jody Kreiman, Bruce R. Gerratt, Norma Antonanzas-Barroso (Div. of Head/Neck Surgery, UCLA School of Medicine, 31-24 Rehab. Ctr., Los Angeles, CA 90095-1794), and Abeer Alwan (UCLA, Los Angeles, CA 90095)

Research investigating the correlation of acoustic measures of noise and the perception of pathological voice quality has consistently demonstrated a moderate association. However, this correlational approach cannot address basic questions concerning their cause and effect relationship, such as how carefully noise must be modeled to preserve the perceived pathological vocal quality, and what particular aspects of the noise are necessary to preserve natural vocal quality. To address these questions, an analysis by synthesis approach was applied to a large sample of natural pathological vowels varying in severity of pathology. Several synthetic versions of each voice were created using an LF source model and a formant synthesizer. Amplitude and spectral characteristics of the noise were varied as necessary. Stimuli were compared perceptually to the original voice and to each other. A discussion will focus on the procedures necessary to adequately model noise in voices with severe vocal pathology, and on the perceptual importance of differences in how vocal noise is synthesized. [Research supported by NIH.]

2pSC31. A perceptual and acoustic study of the imitated voice. Kirk P. H. Sullivan (Dept. Ling., Umeå Univ., S-901 87 Umeå, Sweden) and Frank Schlichting (Hamburg Univ., D-20144 Hamburg, Germany)

The belief that an individual can discriminate between different voices from memory is central to the concept of the voice line-up, the aural analogue of the police identification parade, and its application in the legal sphere. This paper examines the impact professional and amateur imitations have on the accuracy of the technique and whether differences in impact can be explained by an examination of vowel space, timing, and $F 0$ values. Three groups of experimental participants heard a series of different voice line-ups. They were instructed to indicate which voice of each series was the voice they had been asked to identify. Not all of the series contained the voice to be identified. Group one was presented lineups containing a high-quality professional imitation. This resulted in a significant degree of confusion between the voice to be identified and the imitation. Group two was presented the same series of line-ups but the professional imitation was exchanged for an amateur one. Group three was presented the same line-ups, but containing another amateur imitation. The amateur imitators failed to cause confusion with the voice they were imitating, yet succeeded, to different degrees, in disguising their natural voices such that their own identity was protected. 
2pSC32. Acoustic analysis of the effects of alcohol on the human voice. Orla M. Cooney, Kevin G. McGuigan, Peter J. P. Murphy (Dept. of Phys., Royal College of Surgeons in Ireland, St. Stephens Green, Dublin 2, Ireland, kmcguigan@rcsi), and Ronan M. Conroy (Royal College of Surgeons in Ireland, Dublin 2, Ireland)

Experiments to determine the physical effects of alcohol on the human voice are reported. Recordings of sustained phonations and prescribed texts were taken from 12 volunteers at various stages of alcohol consumption, to determine whether a significant change in voice frequencies occurs when alcohol has been consumed. No association between the fundamental frequency of a sustained phonation and level of alcohol consumption is observed (regression coefficient $=0.146, t$ ratio $=0.28, d f=71, p=0.781$ ). Similar results for the higher formant frequencies are also observed. The effect of alcohol on duration of speech is also investigated. Prescribedtext, sentence duration is found to increase at the rate of $6.4 \%$ (standard error $=0.5 \%, p<0.0001)$ per $\mathrm{ml}$ of alcohol consumed per kilogram of body weight. There is no evidence that the sex of the speaker is related to the effect of alcohol on sentence duration. Acoustic analysis of fixed-text voice recordings is therefore not a suitable method of determining whether a person is alcoholically intoxicated. [This research was supported by the Research Committee of the Royal College of Surgeons in Ireland.]
2pSC33. The use of random spliced speech for the recognition of familiar voices. Ricardo M. Figueiredo (Dept. of Legal Medicine, UNICAMP, CX. POSTAL 6153, CEP 13084, Campinas, Brazil, ricfig@turing.unicamp.br)

Various techniques for experimental speech signal manipulation to test recognition of familiar voices have been used, but random spliced speech (RSS) has not been tried. This procedure, which consists of randomly recombining digitally segmented speech signals, offers the advantage of more natural-sounding speech and decreased risk of creating artifacts interfering with perception. Although intelligibility is also destroyed by this technique, RSS preserves the spectral cues related to voice quality, as well as $F 0$ level and range. In the present experiment, various $3.5 \mathrm{~s}$ RSS samples of 11 males were presented to 34 listeners, each familiar with only one of the voices. In a recognition task with forced yes/no responses, the index of correct identification was well above chance. An analysis of misindentifications revealed that proximity of $F 0$ means led to more errors than did similarity of long term average spectra. Since speakers with $F 0$ mean less than 2.8 semitones apart were frequently confused, it seems that in such an experimental situation the listeners may be forced to rely on isolated cues, such as $F 0$; this may explain the high number of false identifications, even though the number of false rejections is practically nonexistent.

TUESDAY AFTERNOON, 23 JUNE 1998

WEST BALLROOM A (S), 2:15 TO 6:20 P.M.

\title{
Session 2pSPa
}

\section{Signal Processing in Acoustics, Architectural Acoustics and Speech Communication: Multichannel Signal Processing for Acoustical Applications}

\author{
David J. Evans, Chair \\ National Institute for Standards and Technology, Building 233, Room A147, Gaithersburg, Maryland 20899
}

Chair's Introduction-2:15

Invited Papers

2:20

2pSPa1. Reconstruction and projection of interior sound fields using a spherical measurement array. Earl G. Williams (Naval Res. Lab., Code 7137, Washington, DC 20375)

A spherical surface populated with microphones is placed within an aircraft fuselage or automotive cabin, and the sound field is determined throughout the interior of the source. This hypothetical experiment is the goal of the measurement system which will be discussed. The output from this spherical antenna is processed using mathematical and signal processing techniques to solve both a forward (projection) and an inverse (reconstruction) problem when no sources are within the array. The forward projection provides the field inside the measurement surface. The field outside is computed by solving an inverse problem providing a reconstruction of pressure and velocity in a spherical region up to the external enclosing boundaries. The accuracy of a proposed measurement system for both the forward and inverse modes of operation will be discussed. This discussion includes number of sensors and sensor placement schemes, and, most importantly, the effect of ambient noise and SNR on the projections and reconstructions, including the maximum reconstruction distances from the measurement sphere which can be obtained. [Work supported by ONR and NASA.]

\section{2:50}

2pSPa2. Multichannel acoustical measurement for sound field mapping. Svend Gade (Brüel \& Kjær Skodsborgvej 307, DK-2850 Nærum, Denmark, sgade@bk.dk) and Jørgen Hald (Brüel \& Kjær, DK-2850 Nærum, Denmark, jhald@bk.dk)

A newly developed sound field mapping method, which uses time-domain holography, is useful for localization, quantification, and ranking of sound generating regions on engines and gearboxes under run-up (i.e., nonstationary) conditions. The technique, which is called nonstationary STSF, requires simultaneous measurement of time histories on a planar surface covering the sound source. Thus subarray scanning as used with the cross-spectral STSF technique cannot be applied and a two-dimensional microphone array with half wavelength microphone separation must be used. Consequences of the need for a full size fixed array system is that it is not feasible for measurements to cover a whole vehicle, except at low frequencies, and for engine measurements a large number of microphone measurement channels is needed to cover a frequency range up to $3 \mathrm{kHz}$. The technique maps all sound field descriptors 
in the near field, but not only as a function of physical location but also as a function of time for transient events. Nonstationary STSF allows source attenuation simulations similar to the cross-spectral STSF technique to be performed. The development of TDH is part of the Brite-Euram project PIANO, dealing with new pass-by noise optimization methods for quiet and economic heavy road vehicles.

3:20

2pSPa3. Signal processing for sound capture. Daniel V. Rabinkin, Richard J. Renomeron, Atul Sharma, and James L. Flanagan (CAIP Ctr., Rutgers Univ., 96 Frelinghuysen Rd., Piscataway, NJ 08854-8088)

High-quality electret microphones and single-chip processors are economical enough to be used in large numbers. This latitude opens opportunities for dynamic source location and sound capture with spatial selectivity in three dimensions. This report discusses algorithms for matched-filter processing of microphone arrays and for coordinate tracking of moving talkers. A prototype conferencing system is demonstrated in which the automatic source locator steers both a video camera and a beam-forming microphone array to capture image and audio from a moving talker. [Components of this research are supported by NSF Contract 397-26740 and DARPA Contract DABT63-93-C0037, the New Jersey Commission on Science and Technology, and the corporate members of the Rutgers Center for Computer Aids for Industrial Productivity (CAIP).]

3:50

2pSPa4. Modeling in-vehicle engine noise. Filip Deblauwe, Patrick Van de Ponseele, and Geert Lowet (LMS Intl., Interleuvenlaan 68, B-3001 Leuven, Belgium, fdb@1ms.be)

Transfer path analysis is accepted as a tool to model or troubleshoot the lower engine orders for in-vehicle noise. The contribution of each force input that is coherent with the engine can be evaluated both for amplitude as well as phase. This allows ranking the individual paths and allows identifying paths that interact (cancel effects). The ability to listen to these contributions, also called partial pressures, is needed for the sound quality evaluation of engine noise, and the next logical step in transfer path analysis. Steady-state applications of time domain transfer path analysis have been reported, but primarily the study of pseudostationary sounds such as engine run-ups was desired. In this document, different methods will be discussed that could enable this type of work. The new developed synthesized order substitution method is presented. This method enables an engine sound engineer to listen to the sound due to a single or a set of noise transfer paths, for a reasonable computation effort.

\section{4:20-4:35 Break}

\section{Contributed Papers}

\section{4:35}

2pSPa5. Localization of acoustic emissions in aerospace structures. Paul J. Wells, Arthur Stephens (Adv. Information Processing Dept., Br. Aerosp. Sowerby Res. Ctr., FPC267, PO Box 5, Filton, Bristol BS12 7QW, UK), and Andy Ibbotson (Br. Aerosp. Airbus, Filton, Bristol, UK)

Health Usage and Monitoring Systems (HUMS) offer the potential to revolutionize the civil aircraft industry's approach to routine scheduled inspections. Developments in acoustic emission measurements allow the direct detection and localization of fatigue damage in complex primary aircraft structures. This information has been used to successfully guide inspections and to locate damage in regions inaccessible to conventional NDT methods. The use of such a system in flight will lead to inspection costs being reduced significantly and will promote confidence in the structural integrity of next generation civil aircraft. This paper will examine the question of the optimum positioning of sensors for a given sample using methods such as genetic algorithms and simulated annealing. The use of dimensional reduction projection techniques is also discussed. In an aerospace structure the propagation of sound will not be isotropic because of the variation in the thickness of the structure. This anisotropy will limit the accuracy of localization unless accounted for. The magnitude of this effect is determined empirically for an aircraft wing panel, and a method for calibrating acoustic emission measurements to minimize the effect of this anisotropy is suggested.

\section{4:50}

2pSPa6. Imaging of supersonic surface fields on a cylindrical shell. Charles N. Corrado, Jr., Daniel T. DiPerna, and Matthew Conti (Eng. Tech. Ctr., 240 Oral School Rd., Mystic, CT 06355-1208)

Conventional imaging techniques represent a radiating body as a distribution of equivalent monopole sources. This paper presents a more rigorous approach for imaging the supersonic components of both surface pressure and normal velocity produced by scattering or radiation from a nominally cylindrical body. The technique employs the Helmholtz integral equation to relate monopole and dipole strengths. A modal expansion of the surface pressure on a baffled cylinder forms the basis for determining an array-weighting function used to estimate the surface fields. The technique makes use of acoustic pressure data measured in a horizontal plane at ranges where the evanescent field is insignificant. No far-field constraints are imposed in the derivation of the method, allowing the measurements to be conducted within the geometric near-field of the body. When the array is located in the far field, this method is shown to yield similar array-weighting functions to a method derived by Williams [E. G. Williams, J. Acoust. Soc. Am. 99, 2022 (1996)]. Mid-frequency, $2 \leqslant k a \leqslant 20$, scattering and radiation data from measurements of a ribbed cylindrical shell are used to illustrate the technique. The intensity images reveal membrane wave and rib scattering processes in both the time and frequency domains. [Work supported by NSWC and ONR.]

\section{5:05}

2pSPa7. Holographic reconstruction of active sources in a threedimensional enclosure. Yang-Hann Kim, Young-Key Kim (Ctr. for Noise and Vib. Control, Dept. of Mech. Eng., Korea Adv. Inst. of Sci. and Technol., Taejon, 305-701, Korea), Byeongsik Ko, and Kangho Ko (Daewoo Motor Co., 199 Chongchon-dong, Pupyong-gu, Inchon, 403-714, Korea)

This study proposes a method to identify active sources. The theory itself does not limit the application of the proposed method, but the main aim has been focused on finding the location of active noise sources and their strengths in an enclosure. The acoustic holography method can be applied to identify active sources, primary sources if one prefers to call them. However, it will depict all sources including imaginary sources, which one may call secondary sources, which are due to reflections on the walls of enclosure. In this study, an attempt has been made to distinguish these two different types of sources. First, boundaries are modeled by the inhomogeneous Robin boundary condition. It makes it possible to represent the boundaries as the combination of general ideal sources and passive boundaries; the ideal sources imply the primary sources, while the 
passive boundaries control the secondary sources. Second, this general boundary condition is combined with the holography method to reconstruct the strength of ideal sources. A technique for estimating surface admittance of passive boundaries is also proposed for a three-dimensional sound field by generalizing that for a duct.

\section{$5: 20$}

2pSPa8. Estimation of far-field pressures from geometric near-field measurements. Daniel T. DiPerna, Charles N. Corrado, Jr. (Eng. Technol. Ctr., 240 Oral School Rd., Mystic, CT 06355-1208, ccorrado@atinc.com), and David C. Warwick (Naval Surface Warfare Ctr., West Bethesda, MD 20817-5700)

Measurements of radiated noise are often made in the geometric near field of a cylindrical radiator to take advantage of high signal-to-noise ratios. At these ranges the far-field beam patterns are not fully established and the corresponding sound pressure levels cannot be determined via a simple range correction. In this paper, three methods of estimating the far field given measurements primarily made in a horizontal plane are presented. All three methods employ propagator matrices to relate surface field quantities to the near- and far-field pressures. The pressures measured over a segment of the receive array are multiplied by a weighting vector determined from the propagator matrices to minimize the mean square error between the actual and estimated far field. One method represents the surface field as a distribution of monopole sources of unknown strength. This method also employs an image source field representation to attenuate surface bounce contributions. The second method represents the surface field in terms of cylindrical harmonics with unknown modal coefficients employed at discretized axial positions along the cylinder. The third method is formulated using unknown modal coefficients derived from a substitution of the spherical harmonic expression of the free-space Green's function in the Helmholtz integral equation.

\section{$5: 35$}

2pSPa9. Feasibility of using imperfect microphone arrays in noise source location. Tarun Bhatt, J. Richard Houghton, and Corinne M. Darvennes (Dept. of Mech. Eng., Tennessee Technolog. Univ., Cookeville, TN 38505, tarun@bigfoot.com)

Microphone arrays are being used in many applications such as noise source location, sound intensity measurement, or acoustic holography. For the purpose of source location using the unwrapped phase method, it is important to use phase-matched microphones. The paper studies the feasibility of replacing phase-matched microphones with an array of unmatched microphones and of finding faulty microphones within the array. For this purpose, two sets of tests were performed. With the first set, the microphone characteristics were measured. An electronic circuit provided time delays, i.e., phase information, between the microphones, and narrow and wideband source signals supplied frequency responses for all the microphones. With the second set of tests, optimization techniques and neural network methods were used to locate a noise source with (1) a matched microphone array, (2) an unmatched array, and (3) an array containing faulty microphones. Comparison of the data from the various microphone arrays is presented in this paper. The feasibility of using unmatched arrays with optimization and/or neural network methods is discussed. It is also demonstrated that a microphone with a significant signal-to-noise ratio and/or a large phase error can be pinpointed within the microphone array.

\section{$5: 50$}

2pSPa10. Superdirectivity design for a sphere-buffled microphone array. Kazuhiko Kawahara and Kimitoshi Fukudome (Dept. of Acoust. Design, Kyushu Inst. of Design, 4-9-1 Shiobaru Minami-ku, Fukuoka City, 815-8540 Japan, kawahara@kyushu-id.ac.jp)

For the signal extraction from a diffuse sound field, the superdirecitonal microphone array seems to be more stable and more effective than the noise canceling microphone array system. In this research, for the sphere-baffled microphone array (SBM), the superdirectivity characteristic was designed by the frequency domain beamforming method and the complex least mean square algorithm. The frequency domain beamforming is a method for a broadband beamforming. There is a possibility of improvement of the directivity frequency characteristic. The SBM is an array which was embedded 36 elements on the rigid diffractive sphere of 8.84 $\mathrm{cm}$ radius. An advantage of SBM is its isotropy. When the directivity characteristic was fixed for a definite direction, only the shift coefficent can be used to change the mainlobe direction. This idea can be easily generalized into the three-dimensional mainlobe control. In a typical design calculation, for the beamwidth of $-3 \mathrm{~dB}, 30^{\circ}$ was achieved at the frequency $2 \mathrm{kHz}$. This characteristic is comparable to that of a larger size microphone, which is called a shotgun microphone.

\section{6:05}

2pSPa11. Beamforming for broadband constant beamwidth through FIR filter and DSP implementation. Yuanliang Ma, Baosong Zhang (Inst. of Acoust. Eng., Northwestern Polytechnical Univ., Xi'an 710072, PROC, ylma@nwpu.edu.cn), and Didier Vray (Creatis, INSA de Lyon, France)

The paper presents a method to design a beamformer for broadband constant beamwidth. This is important in sonar systems for sea bottom observation and classification applications. For a given frequency band, a number of frequency points are chosen to represent the response. For each frequency point, a beamforming weighting vector can be deduced to satisfy a given beamwidth requirement by existing methods, for example, Chebyshev shielding coefficients can be deduced for a linear array. For a broadband array of $N$ elements, a $N^{*} M$ matrix can be obtained if $M$ frequency points are chosen. Each row of the matrix represents a frequency response of an array element, which can be satistied by a FIR filter designed by model-reference adaptive techniques. The sum of outputs from a bank of $N$ FIR filters provides a beamformed output and so it is very handy to be implemented by DSP hardware. A design example shows that the results are satisfactory. 


\title{
Session 2pSPb
}

\section{Signal Processing in Acoustics: Implementation Issues of Acoustical Signal Processing in Real Time Systems II}

\author{
Christian P. de Moustier, Chair \\ Scripps Institute of Oceanography, Marine Physical Laboratory, 9500 Gilman Drive, La Jolla, California 92093-0205
}

\section{Contributed Papers}

\section{2:15}

2pSPb1. FFT beamforming with cylindrical arrays: Application to the Toroidal Volume Search Sonar. Christian P. deMoustier and Timothy C. Gallaudet (Marine Physical Lab., Scripps Inst. of Oceanogr., 9500 Gilman Dr., La Jolla, CA, 92093-0205)

FFT beamforming is typically applied to arrays of coplanar and equally spaced elements. For curved arrays, conventional delay and sum beamforming should be the norm because the elements are not coplanar and the projection of their position on a tangent plane yields unevenly spaced elements. However, with appropriate phase correction coefficients, FFT beamforming can be applied to the elements contained in a $90^{\circ}$ sector of a cylindrical array if beams formed within $\pm 35^{\circ}$ about broadside are kept. Within this sector results are essentially identical to those obtained via conventional beamforming but the FFT technique is more efficient computationally and better suited to real-time applications. The error bounds associated with FFT beamforming on cylindrical arrays are discussed and we present applications of the technique to data collected with the Toroidal Volume Search Sonar by the Coastal System Station, Panama City, FL. [Work sponsored by ONR-NRL (Contract No. N00014-96-1G913).]

\section{2:35}

2pSPb2. Acoustic source estimation based on physical modeling and optimization algorithm. Aggelos J. Amditis, K. S. Nikita, and Nikoloas K. Uzunoglu (Dept. of Elec. and Comput. Eng., Natl. Tech. Univ. of Athens, 15773 Zografou Cambus, 9 Iroon Polytechniou St., Zografou, Athens, Greece)

The propagation of acoustic waves in the sea is analyzed by employing a horizontally stratified model for the sea medium with an arbitrary number of layers. Through this physical model, the acoustic field distribution, originating from an elementary source at an arbitrary location point inside the sea medium, is calculated by implementing a Green's function approach. The proposed optimization algorithm is based on the minimization of the quadratic error function between estimated source signal values and signal values corresponding to known acoustic sources for the frequency content of the measured signal. Initial source estimation is based on the measured signal and the transfer function obtained by the above described propagation model. The minimization of the error function with respect to the model parameters described is carried out independently for each signal, corresponding to a known source, by employing the downhill-simplex minimization method. The measured signal is positively recognized as one corresponding to a known acoustic source if the minimization procedure results in an error value less than a predefined limit.

\section{2:55}

2pSPb3. Robust Kalman-based time-varying DOA estimation of impulsive noise. Han-Su Kim and Koeng-Mo Sung (School of Elec. Eng., Seoul Natl. Univ., Seoul 151-742, South Korea)

This paper presents the robust Kalman-based method which is modified with a Huber function for estimating time-varying DOA (direction of arrival) under an impulsive noise environment. The Kalman-based algo- rithm can estimate the proper parameter in the additive white Gaussian noise. But it is well known that the performance of the Kalman algorithm degrades seriously when the input or the estimation error contain impulsive noise (burst outliers) which can arise frequently in many practical applications. The proposed algorithm has the robustness over the impulsive noise by putting bounds to the derivative of its cost function with respect to the estimation error, using Huber function. It is known in the theory of robust estimation that if the influence function is bounded, the estimator is robust. Simulations show that the performance of the proposed algorithm is less vulnerable to the impulsive noise than the conventional Kalman algorithm.

\section{3:15}

2pSPb4. A universal parallel sonar signal processing system. Lifu You and Enfang Sang (Dept. of Underwater Acoust. Eng., Harbin Eng. Univ., Harbin 150001, PROC, euipc@ public.hr.hl.cn)

A modern signal processing algorithm has been widely used in the sonar system, which has improved the sonar system features, but has also given the sonar signal processing system more compute burden in the meantime. Parallel signal processing technology is a solution. A universal parallel signal processing module is designed, which is based on the VME bus, so it is convenient to get a more powerful processing system. This module is comprised of four TMS320C40 digital signal processors. As examples, some basic sonar signal processing algorithms such as FFT, beamforming, etc. have been implemented using this system. The efficiency and speedup are given in this paper. This parallel signal processing system is suitable for many kinds of sonar systems.

\section{3:35}

2pSPb5. Maximum likelihood track-before-detect matched-field beamforming with SWELLEX data. Kerem Harmanc1, Joseph Tabrikian, and Jeffrey L. Krolik (Dept. of Elec. and Comput. Eng., Duke Univ., Durham, NC 27708-0291)

Moving source localization methods are seriously limited at low signal-to-noise ratios because the signal energy becomes spatially spread over long observation times. In this paper, the integration time available to matched-field beamformers is extended by incorporating a priori statistical knowledge of the source dynamics. This is accomplished by first showing that the well-known minimum variance beamformer output power is essentially the log-likelihood function with respect to source location under a model consisting of a single target in an unknown but structured noise field. This likelihood function for a single frame of data may be combined with the conditional distribution of the current target position given its previous location to obtain a maximum-likelihood (ML) trackbefore-detect processor. In cases where the sample covariance matrix is ill-conditioned, derivation of a constrained ML estimate assuming known diffuse noise level is given. Results are presented using vertical hydrophone array data for an underwater acoustic source off the coast of southern California. Comparisons with conventional MV matched-field beamforming demonstrate that incorporating source dynamics into the processor improves its ability to maintain the target track even in the presence of interference. 


\title{
Session 2pUW
}

\section{Underwater Acoustics: Scattering and Reverberation (Précis Poster Session)}

\author{
Dajun Tang, Chair \\ Applied Physics Laboratory, University of Washington, 1013 NE 40th Street, Seattle, Washington 98105-6698 \\ Chair's Introduction-2:15 \\ Contributed Papers
}

In this session, an oral summary of each paper will be presented followed by a poster session. All posters will be on display, and all contributors will be at their posters, from 4:00 p.m. to 5:30 p.m.

2:20

2pUW1. The effects of bandwidth, dispersion, and correlation length scale on shallow-water reverberation. Kevin D. LePage (SACLANT Undersea Res. Ctr., Viale San Bartolomeo 400, 19138 La Spezia, Italy)

The ensemble average of the short-time average of the monostatic reverberation power expected in a shallow-water waveguide is obtained explicitly in the parameters of modal phase speed, slowness, dispersion, and source bandwidth under the assumption of a Gaussian source envelope and a two-dimensional Gaussian spatial scatterer correlation function. Results show that in the limiting case of very narrow bandwidths, resonant scattering is expected, yielding lower backscattered levels, and significant propagation structure is superimposed on the backscatterer time series, consistent with the two way TL to the scattering patch at the center frequency. In the limit of patch size small compared to the correlation length scale, higher backscattered levels, consistent with the scattering strength of the individual scatterers themselves, are expected, and the superimposed propagation structure is reduced due to the spatial decorrelation of the patches interrogated by the individual modes. Expressions for the time varying vertical coherence are also obtained in this framework.

\section{2:25}

2pUW2. High-frequency acoustic scattering from thermally generated turbulence. John Oeschger (Coastal Systems Station, Naval Surface Warfare Ctr., Dahlgren Div., Panama City, FL 32407-7001, oeschger_john@ccmail.nscs.navy.mil)

The results of a laboratory experiment are presented for the case of high-frequency broadband multistatic acoustic scatter from a thermally generated buoyant plume. The dominant scattering mechanism is changes in the relative compressibility, which is related to the temperature difference from ambient. Far-field weak scattering theory is used to describe the scattering process. This results in a simple relationship between the complex acoustic scatter and the one-component, three-dimensional Fourier transform of the temperature field measured at the scattering producing Fourier component $\mathrm{K}$, defined as the Bragg wave number. This wave number is the magnitude of the difference wave vector between the scattered and incident wave vectors. Theory predicts for measurements made in the same scattering direction that spectral estimates for overlapping Bragg wave numbers be identical. Utilizing data collected from simultaneous multiple bistatic configurations with parallel Bragg wave vectors yields a more fully resolved spectral estimate of the scattering producing temperature field. A time series of the spectral estimate of the turbulent temperature field is shown for a three-channel bistatic common Bragg direction measurement. The results indicate agreement between the spectral estimates in the regions of overlapping Bragg wave numbers, validating the theoretical description. The time series also shows strong, yet highly variable nulls in the wave-number spectrum of the turbulence. [Work supported by ONR.]
$2: 30$

2pUW3. Range spread measurement of underwater scatterers and channels using active sonar probing signals and a cross-correlation process. James J. Kisenwether and Dennis W. Ricker (Autonomous Control and Intelligent Systems Div., Appl. Res. Lab., Penn State Univ., State College, PA 16804)

A method for measuring range spread of underwater scatterers and channels based on the cross-correlation processing of the tones of a multicomponent waveform has been developed. The correlation terms which comprise the measure are samples of the time-frequency correlation functions (TFCF) of scatterers and channels. The measure has been used to characterize the time-frequency spread and represents the signal coherence. This paper will provide a review of two frequency correlation function representations and their characterization using the aggregate coherence measure, termed correlation mass. The sensitivity of this measure as a function of waveform parameters, scatterer structure, and measurement platform motion will be defined and its robustness on a multiping basis assessed. It will be shown that this measure can be decomposed into correlated components resulting from interaction with a scatterer and a channel. Essentially the composite correlation is obtained by sampling the product of the scatterer and channel TFCF's. Data from in-water experiments and digital sonar simulations will be presented which validates the linear systems model of the composite scattering process. [Work supported by ONR.]

\section{2:35}

2pUW4. Target size measurements via specular echo spectrum. Jerome Mathieu, Patrick Schweitzer, Etienne Tisserand, Serge Weber (L.I.E.N. (Electron. Instrumentation Lab. of Nancy), Univ. Henri Poincare-Nancy 1, BP 239, 54506 Vandoeuvre-les-Nancy Cedex, France, mathieu@lien.u-nancy.fr), and Serge Gauthier (G.E.M.C.E.A., 54500 Vandoeuvre-les-Nancy, France)

Ultrasound principles actually used to determine experimentally the size A of solid targets are based on measurement of scattering attenuation. The principal limit of this method comes from the difficulties in identifying excess attenuation due to scattering. This paper presents a new method to determine the size A of a target by means of specular echo spectrum. When an immerged (in water) target is insonified with a very brief ultrasound pulse, the backscattered echo contains two parts temporally distinguishable. The first part is the specular echo. The specular echo target spectrum St depends on the form function $\mathrm{F}$ and A, but also of the measuring chain response. So this method includes a gauging step which consist to calculate the spectrum Sb of a block's echo. It is also showed that $\mathrm{Sb} / \mathrm{St}$ (which is noted Fmes) is proportional to $\mathrm{F}$ in the bandwidth measuring chain B. The comparison of Fmes and F gives the bandwidth B in 
terms of KA, where $\mathrm{K}$ is a known wave number. Thus $\mathrm{A}$ is deduced by comparing Fmes and F. The most interesting advantage of this method is its independence towards attenuation. Some results are presented. layered media, this model allows one to discuss the various components and effects featured in the resultant backscattering strength. It is finally compared to acoustical data recorded in areas where some ground truth is available.

\section{2:55}

2pUW8. Measurement at low ultrasonic frequencies of absorption due to suspended particulate matter. Niven R. Brown, Timothy G. Leighton (Inst. of Sound and Vib. Res., Univ. of Southampton, Southampton SO17 1BJ, UK, nrb@isvr.soton.ac.uk), Simon D. Richards, and Tony D. Heathershaw (Defence Evaluation and Res. Agency, UK)

The visco-thermal absorption of sound by suspended particulate matter can be reliably measured using a reverberation technique. This absorption may have an adverse effect on the performance of sonars operating at low ultrasonic frequencies in coastal waters where suspensions are often present in significant concentrations. A series of experiments has been performed to study the viscous absorption by suspensions in the frequency range of $50-150 \mathrm{kHz}$ by taking the difference in reverberation times of a volume of water with and without particles. Measured attenuation agrees reasonably well with that predicted by theory for concentrations above 0.5 $\mathrm{g} / \mathrm{l}$. A number of interesting phenomena have also been observed which are related to the measurement system and the turbulent flow required to keep the particulate suspended. For accurate measurement, losses at the container walls must be minimized. Although there are small losses at the surface by transmission, along with losses due to absorption in the acoustic boundary layer, the presence of bubbles and dissolved gas, and turbulence, the relative measurement gives a good assessment of the absorption by the particles. These effects are discussed and results for particulate absorption are presented.

\section{3:00}

2pUW9. Turbidity in future high-frequency sonar performance models. Simon D. Richards (Defence Evaluation and Res. Agency, UK), Niven R. Brown, and Timothy G. Leighton (Univ. of Southampton, Southampton, UK)

Current sonar performance models are incapable of accurately predicting the performance of high-frequency sonars in highly variable turbid coastal waters. There is therefore a requirement for improved models incorporating the additional effects in such environments. Turbid coastal waters are characterized by relatively high levels of suspended particulate matter, the presence of which leads to increased attenuation through viscous absorption and scattering, leading to a significant reduction in the detection range of high-frequency active sonars at moderate concentrations. The additional attenuation mechanisms have been incorporated into a propagation-loss model and the effect on detection range has been investigated. The additional attenuation processes are influenced by the ambient temperature, pressure, and salinity, and the effects of these parameters on the total attenuation in seawater containing suspended mineral particles has also been investigated. The results presented demonstrate that the effects of suspended particulate matter should be included in future high-frequency sonar performance prediction models in turbid environments. Temperature is found to be an important factor influencing the attenuation, and the local temperature should therefore be used in performance calculations. [Published with the permission of the Controller of Her Britannic Majesty's Stationery Office.]

\section{3:05}

2pUW10. Spectral diffusion of seismo-acoustic waves in shallow water. Robert I. Odom (Appl. Phys. Lab., Univ. of Washington, 1013 NE 40th St., Seattle, WA 98105) and Valerie I. Peyton (Yale Univ., P.O. Box 208109, New Haven, CT 06511)

The propagation of seismo-acoustic waves in a strongly forward scattering medium is modeled as an energy diffusion process satisfying a convection-diffusion equation in slowness space [Odom and Mercer, Geophys. J. Int. 127, 111-124 (1996)]. No energy propagates with a slowness greater than the Stoneley/Scholte wave slowness, and therefore must sat- 
isfy a zero flux boundary condition at the maximum slowness. At a slowness equal to $1 /$ (minimum shear speed), energy is lost by radiation to shear waves, and for finite frequencies satisfies a mixed boundary condition in slowness space. Maupin [Geophys. J. Int. 126, 762-780 (1996)] has recently shown how the branch line integral for the elastic continuum can be written as a dyad, which does not suffer from common problems with other formulations employing leaky modes. Maupin's dyadic representation for the elastic wave continuum is employed to derive the proper mixed boundary condition and illustrate its effect on the diffusion of seismo-acoustic wave energy in a shallow water waveguide. [Work supported by ONR.]

\section{3:10}

2pUW11. Experimental verification of the deformed cylinder scattering model and its application for the calculation of backscattering from fish. Kouichi Sawada (Natl. Res. Inst. of Fisheries Eng., Ebidai, Hsaki, Kashima, Japan), Zhen Ye (Natl. Central Univ., Chung-li, Taiwan, R.O.C.), and Masahiko Furusawa (Tokyo Univ. of Fisheries, 4-5-7 Konan, Minato, Tokyo, Japan)

The dorsal aspect target strength pattern of straight cylinders made of polystyrene (EPS) are measured and compared with the deformed cylinder scattering model (DCM) proposed by Ye [Zhen Ye, J. Acoust. Soc. Am. 102, 877-884 (1997)] in the range of the tilt angle from $-50^{\circ}$ to $50^{\circ}$. Both experiments and the theory agreed very well for the wide range of tilt angle. The deformed cylinder model is also applied to predict the target strength pattern of Ayu (Plecoglossus altivelis) which is a river fish with an open swimbladder. The dorsal target strength of Ayu is measured by keeping it in alive condition. Then its swimbladder's shape is observed using a soft $\mathrm{x}$-ray system and digitized. Digitized data is used for the calculation on the basis of the deformed cylinder model and also the shape of the swimbladder is reproduced carving a rectangular solid of EPS by hand. The measured TS pattern of alive Ayu, the physical EPS model of swimbladder, and the DCM calculation agreed very well. Expanded polystyrene is a useful material to verify the scattering theory of the gas model and can become an artificial fish model with a swimbladder. Scattering from a complicated shape as a fish is predictable using DCM.

\section{3:15}

2pUW12. Comparison between subcritical penetration models and in situ data. Alain Maguer, Eric Pouliquen, Edoardo Bovio, Warren L. J. Fox, and Henrik Schmidt (NATO SACLANT Undersea Res. Ctr., Viale S. Bartolomeo 400, 19138 La Spezia, Italy)

This paper presents the recent results of a study whose overall objectives are to determine the mechanism(s) contributing to anomalous highfrequency sound penetration into sediments, and to quantify the results for use in sonar performance prediction for the detection of buried objects. To date, several mechanisms have been hypothesized in order to explain this phenomenon, the most frequently mentioned being: (a) the existence of a Biot slow wave in the sediment, (b) surface roughness, and (c) scattering of the evanescent wave by volume inhomogeneities within the sediment. In situ acoustic measurements were performed on a sandy bottom whose geoacoustic properties were carefully identified. A parametric array mounted on a tower moving on a rail was used to insonify hydrophones located above and below the sediment interface. An extensive data set covering a wide range of grazing angles (both above and below the estimated critical angle) and frequencies $(2-50 \mathrm{kHz})$ was acquired and processed. The results are compared to models for the three mechanisms stated in an attempt to explain the anomalously high sound-pressure levels measured. Dependence on frequency, depth into the sediment, and grazing angle is explored.
2pUW13. Subcritical penetration into a viscoelastic solid due to interface roughness: Laboratory experiment and perturbation theory model. Garfield R. Mellema, Terry E. Ewart, and Kevin L. Williams (Appl. Phys. Lab., College of Ocean and Fishery Sci., and Dept. of Elec. Eng., Univ. of Washington, Seattle, WA 98105, mellema@apl.washington.edu)

The propagation of acoustic energy into sub-bottom sediments at grazing angles below the compressional critical angle has been well documented and various mechanisms to explain this propagation have been proposed. Due to the complexity of the in-situ environment the propagated energy might not be the result of a single mechanism, but a sum of a combination of several mechanisms. In order to better consider the validity of one of the more prominent mechanisms, rough surface scattering, a simplified laboratory environment has been constructed in which to make measurements of penetration due to interface roughness. In this environment, the sediment has been replaced by a homogenous viscoelastic solid. To preserve the uniformity of the solid, both the transmitter and the receiver are located in the water column, where their radiation patterns are well characterized. Use of an automated positioning and measurement system and a large interface area ensures that the series of measurements are both repeatable and statistically meaningful. A perturbation theory model has been constructed for comparison with the measurements. In the experiment its accuracy is evaluated as the surface roughness is increased through the limit of perturbation theory. [Work supported by ONR.]

\section{3:25}

2pUW14. Aspects of a damped surface wave in the Fourier diamond spaces. New surface wave analysis method (SWAM). Loï Martinez, Jean Duclos, and Alain Tinel (LAUE, Pl. R. Schuman, BP 4006, 76610 Le Havre Cedex, France)

A damped surface wave (complex wave number $K=K^{\prime}+j K^{\prime \prime}$ ) is described by its space-frequency representation $S(x, \omega)=\exp (j K(\omega) x)$. The wave-number-frequency representation $K \operatorname{si}(k, \omega)$ is the spatial Fourier transform of $S(x, \omega)$. A $K s i$-cut versus $k$ is a $k$-Breit Wigner form (square modulus maximum for $k=K^{\prime}, K^{\prime \prime}$ half-width). A $K s i$-cut versus $\omega$ is then shown to be an $\omega$-Breit Wigner form (square modulus maximum for $\omega=\Omega^{\prime}$, with an $\Omega^{\prime \prime}$ half-width). New interesting properties are found: the phase velocity is $C_{p}=\Omega^{\prime} / K^{\prime}$ and the group velocity is $C_{g}=\Omega^{\prime \prime} / K^{\prime \prime}$. The wave-number-time representation $N(k, t)$ is the inverse frequency Fourier transform of $K \operatorname{si}(k, \omega) . N(k, t)$ is then shown to be $N(k, t)=\exp (j \Omega(k) t)$. The space-time representation $s(x, t)$ is obtained by inverse frequency (respectively, wave number) Fourier transform of $S(x, \omega)$ [respectively of $N(k, t)]$. The four spaces $s, S, K s i$, and $N$ are the four Fourier diamond spaces. Used on $s(x, t)$ experimental datas, the two cuts properties are the basis of the surface wave analysis methods (SWAM). Those methods identify the complex $K$ (respectively, $\Omega$ ) using $k$-ARMA (respectively $\omega$-ARMA) models. Using SWAM on a cylindrical shell, the $A$-wave is experimentaly fully characterized. The agreement with theoretical results is excellent.

\section{$3: 30$}

2pUW15. Aspects of cylindrical shell resonances in the Fourier diamond spaces. Use of surface wave analysis methods (SWAM) on experimental or numerical datas. Loï Martinez, Jean Duclos, and Alain Tinel (LAUE, Pl. R. Schuman, BP 4006, 76610 Le Havre Cedex, France)

For an attenuated surface wave (wave number $K=K^{\prime}+j K^{\prime \prime}$ ), the resonant space-frequency $S(x, \omega)$ representation of a cylindrical shell is performed versus the angular position $x$. In this space, the MIIR properties are demonstrated. The resonant wave number-frequency representation $K \operatorname{si}(k, \omega)$ is then obtained by spatial Fourier transform of $S(x, \omega)$. This two-dimensional second space clearly separates clockwise and anticlockwise propagating waves. The first ones are observed in the positive $k$ 
values of $K s i$, and the second ones in the negative $k$ values of $K s i$. The modulus of $K s i$ is maximum each time that the resonant condition is encountered [ $\omega$ so that $K^{\prime}(\omega)$ is integer]. As new results, the k cut of Ksi reveals the Sommerfeld Watson aspect of the problem and SWAM identify the complex $K$. On an $\omega$ cut of $K s i$, SWAM identify the RST aspect of the target (and a little more): complex frequency resonances $\Omega=\Omega^{\prime}$ $+j \Omega^{\prime \prime}$ for real modes $n$. SWAM is performed on experimental datas and on numerical form functions. The agreement with theoretical results obtained by RST is very good.

\section{3:35}

2pUW16. New experimental characterization of a resonance: Identification of the mode number using the Argand diagram and the GTD approach. Serge Derible, Jean-Marc Conoir, Jean-Louis Izbicki, and Pascal Rembert (LAUE, Universite du Havre, Pl. R. Schuman, 76610 Le Havre, France)

Until now, the mode $\mathrm{n}$ of vibration of an immersed cylindrical shell, associated to the reemission of a peripheral wave, using an incident quasimonochromatic plane wave is performed by carrying out the method of isolation and identification of resonance. The new method proposed here involves only the FFT of the whole pressure signal backscattered at normal incidence by the shell, normalized using the FFT of the incident wideband signal. At a resonance frequency, corresponding to an unknown value of $\mathrm{n}$, the background phase $\varphi_{\exp }$ is measured from the Argand diagram of the scattered pressure. It corresponds to the argument of the pressure at the resonance frequency. The geometrical theory of diffraction allows us to connect the background phase $\varphi_{\mathrm{GTD}}$ to the mode number $\mathrm{n}$. This mode is then deduced by minimizing the difference between $\varphi_{\exp }$ and $\varphi_{\mathrm{GTD}}$. Experiments are carried out for $A$ and $S_{0}$ resonances. Then, with a single backscattered signal, it is possible to obtain the frequency, the width, and the mode of the resonance.

\section{3:40}

2pUW17. Scholte wave dispersion by rippled liquid/solid interface topography. Jacques R. Chamuel (Sonoquest Adv. Ultrason. Res., P.O. Box 81153, Wellesley Hills, MA 02181-0001)

The effect of sand ripples on seismoacoustic waves is not yet understood. Quantitative results are presented characterizing the dispersion of Scholte waves propagating along a rippled surface of an immersed "soft" solid half-space. One model has a sinusoidal profile with a ratio of corrugation amplitude to wavelength of 0.25. The observed Bragg frequency components of broadband transient received waveforms are shifted by $180^{\circ}$ as the receiver is displaced one cycle along the corrugation, however, the other frequency components are delayed by a smaller amount following the dispersion characteristics. Scholte and Rayleigh wave experimental dispersion graphs obtained from "soft" solid models are compared with replotted scaled numerical results on Rayleigh wave dispersion along a "hard" corrugated solid half-space [Glass et al., "Propagation of Rayleigh surface waves across a large-amplitude grating," Phys. Rev. B 24, 6843-6861 (1981)]. The findings reveal that a rippled "soft" liquid/solid interface can decrease the velocity by more than $70 \%$ of high-frequency Scholte wave components propagating normal to the ripples. The accuracy of acoustic inversion and detection of buried objects depends on predicting the effect of seafloor topography. [Work supported by ONR.]
3:45

2pUW18. Scatterer depth estimation using broadband active matched field processing. Brian K. Jennison (Loyola College, Dept. of Elec. Eng., 4501 N. Charles St., Baltimore, MD 21210-2699), C. Allan Boyles, and Kevin J. McCann (Johns Hopkins Univ., Laurel, MD 20723-6099)

The reduction of false alarms caused by boundary reverberation is one of the major challenges confronting active acoustic systems used for antisubmarine warfare. Scatterer depth serves as a powerful discriminant for distinguishing false target echoes reflecting from the surface or the bottom from actual targets located within the water column. Active matched field processing provides a technique for estimating the scatterer depth by comparing the measured acoustic data with a series of modeled replicas generated assuming different scatterer depths. This paper considers active matched field processing to estimate the depth of a scatterer (range is assumed known from the delay time of the echo) using a single receive hydrophone. Since matched field processing is sensitive to environmental mismatch, the search space is expanded to include both the scatterer depth and the unknown environmental parameters. Simulated annealing is used to efficiently search the parameter space for the optimal solution. A novel cost function, based solely on multipath arrival times rather than the relative multipath amplitudes, is introduced to make the search robust to uncertainties in scatter characteristics. Simulation results demonstrate the effectiveness of the technique to accurately estimate scatterer depth with limited environmental data.

\section{3:50}

2pUW19. The detection of cylindrical objects of low acoustic contrast buried in the seabed. Ruthven C. Evans and Timothy G. Leighton (Inst. of Sound and Vib. Res., Univ. of Southampton, Highfield, Southampton SO17 1BJ, UK, ecpr@isvr.soton.ac.uk)

It is anticipated that the next generation of submarine fiber-optic telecommunications cables will be invisible to present-day detection systems. In cooperation with Cable and Wireless (Marine) Ltd., research has been conducted into the remote detection of buried objects of low acoustic contrast, with particular emphasis given to the detection of buried cables. Specifically, this has concerned the design of a detection system that can reliably discriminate a small diameter ( $\mathrm{cm}$ scale), cylindrical target buried to a depth of up to $1 \mathrm{~m}$ below the seabed from a range of $1 \mathrm{~m}$ above. A purpose-built, laboratory-scale, automated sensing system comprising a bistatic arrangement of adjustable, focused transducers is described. This apparatus has been used successfully in the high-resolution imaging of a range of buried objects, proving an acoustic detection system to be a feasible solution. Recent experimental investigations have focused on two areas: Waveform optimization techniques, to maximize seabed penetration and target interaction, and matched filtering and clutter reduction techniques, to optimize the detection system to the acoustic signature of a particular buried object. Results are presented.

\section{3:55}

2pUW20. Characteristic of a semiburied object in shallow water. Shi-e Yang and Xiukun Li (Dept. of Underwater Acoust. Eng., Harbin Eng. Univ., 150001 Harbin, PROC)

The impulse reflection of a semiburied object is strongly complicated due to a multipath channel effect in a shallow-water environment, and usually also mixed with unavoidable bottom reverberation. An efficient method of signal processing in order to obtain necessary characteristics of the object, which can be used for object identification, is discussed, and several experimental examples are given. 


\title{
Session 2pPLb
}

\section{Plenary Lecture}

William A. Yost, Chair

Loyola University, Parmly Hearing Institute, 6525 West Sheridan Drive, Chicago, Illinois 60626

\author{
Chair's Introduction-5:00
}

Invited Paper

\section{5:05}

2pPLb1. Representation of complex spectra in the peripheral auditory system. Eric D. Young, Barbara M. Calhoun, Jane Yu (Dept. Biomed. Eng. Ctr. Hearing Sci., Johns Hopkins Univ., 720 Rutland Ave., Baltimore, MD 21205, eyoung@bme.jhu.edu), and Israel Nelken (Hebrew Univ.—Hadassah Med. School, Jerusalem, Israel)

The representation of acoustic stimuli in the auditory periphery is tonotopic; frequency is mapped onto a place in the array of auditory neurons and the stimulus energy at each frequency is represented by the activity of neurons at the corresponding place in the neural array. Despite this generality, the details of the process can be complex, in part due to nonlinearities in neural processing. In the dorsal cochlear nucleus (DCN), for example, neurons give the same response to an energy peak in the spectrum as they do to an energy notch. Because of the nonlinearities, it may not be possible to predict the responses to arbitrary spectral shapes from responses to tones. In the DCN, a physiological model which accounts for the nonlinearities has been developed, but this method is not generalizable. A method for characterizing receptive fields for broadband stimuli has been developed based on the presentation of ensembles of stimuli with random spectral shapes; a neuron's sensitivity to a particular frequency is obtained from the correlation between the neuron's rate and the stimulus energy at that frequency. Because this method uses complex stimuli, it should better predict responses to complex stimuli of similar bandwidths. [Work supported by NIDCD.] 